\title{
Design, Fabrication, and Control of an Upper Arm Exoskeleton Assistive Robot
}

\author{
Reza Shisheie
}

Follow this and additional works at: https://researchrepository.wvu.edu/etd

\section{Recommended Citation}

Shisheie, Reza, "Design, Fabrication, and Control of an Upper Arm Exoskeleton Assistive Robot" (2014). Graduate Theses, Dissertations, and Problem Reports. 6636.

https://researchrepository.wvu.edu/etd/6636

This Thesis is protected by copyright and/or related rights. It has been brought to you by the The Research Repository @ WVU with permission from the rights-holder(s). You are free to use this Thesis in any way that is permitted by the copyright and related rights legislation that applies to your use. For other uses you must obtain permission from the rights-holder(s) directly, unless additional rights are indicated by a Creative Commons license in the record and/ or on the work itself. This Thesis has been accepted for inclusion in WVU Graduate Theses, Dissertations, and Problem Reports collection by an authorized administrator of The Research Repository @ WVU. For more information, please contact researchrepository@mail.wvu.edu. 


\title{
Design, Fabrication, and Control of an Upper Arm Exoskeleton Assistive Robot
}

\author{
Reza Shisheie
}

\author{
Thesis submitted \\ to the College of Engineering and Mineral Resources \\ at West Virginia University \\ in partial fulfillment of the requirements for the degree of \\ Master of Science in \\ Mechanical Engineering
}
Larry. E . Banta, Ph.D., P.E., Chair
Marvin Cheng, Ph.D.
Valeriya Gritsenko, Ph.D.

Department of Mechanical and Aerospace Engineering

\author{
Morgantown, West Virginia \\ Spring 2014
}

KEYWORDS: Exoskeleton Robotics, Twisted String Actuation, Master-Slave Control Copyright 2013 Reza Shisheie 


\section{ABSTRACT \\ Design, Fabrication, and Control of an Upper Arm Exoskeleton Assistive Robot}

\section{Reza Shisheie}

Stroke is the primary cause of permanent impairment and neurological damage in the United States and Europe. Annually, about fifteen million individuals worldwide suffer from stroke, which kills about one third of them. For many years, it was believed that major recovery can be achieved only in the first six months after a stroke. More recent research has demonstrated that even many years after a stroke, significant improvement is not out of reach. However, economic pressures, the aging population, and lack of specialists and available human resources can interrupt therapy, which impedes full recovery of patients after being discharged from hospital following initial rehabilitation. Robotic devices, and in particular portable robots that provide rehabilitation therapy at home and in clinics, are a novel way not only to optimize the cost of therapy but also to let more patients benefit from rehabilitation for a longer time. Robots used for such purposes should be smaller, lighter and more affordable than the robots currently used in clinics and hospitals. The common human-machine interaction design criteria such as work envelopes, safety, comfort, adaptability, space limitations, and weight-to-force ratio must still be taken into consideration.

In this work a light, wearable, affordable assistive robot was designed and a controller to assist with an activity of daily life (ADL) was developed. The mechanical design targeted the most vulnerable group of the society to stroke, based on the average size and age of the patients, with adjustability to accommodate a variety of individuals. The novel mechanical design avoids motion singularities and provides a large workspace for various ADLs. Unlike similar exoskeleton robots, the actuators are placed on the patient's torso and the force is transmitted through a Bowden cable mechanism. Since the actuators' mass does not affect the motion of the upper extremities, the robot can be more agile and more powerful. A compact novel actuation method with high power-toweight ratio called the twisted string actuation method was used. Part of the research involved selection and testing of several string compositions and configurations to compare their suitability and to characterize their performance. Feedback sensor count and type have been carefully considered to keep the cost of the system as low as possible. A master-slave controller was designed and its performance in tracking the targeted ADL trajectory was evaluated for one degree of freedom (DOF). An outline for proposed future research will be presented. 


\section{Acknowledgment}

My name appears on the cover of this thesis as the author; however, a great many people accompanied me in completion of this work. I owe my gratitude to all those people, who have made this thesis possible and because of whom my graduate experience has been one that I will cherish forever.

My deepest gratitude is to my advisor and friend Dr. Larry E. Banta, a great teacher and an advisor, who was abundantly helpful and offered priceless assistance, support, and guidance to accomplish this research project. I hope that one day I would become as good an advisor to my students as Larry has been to me.

I would like to express my sincere gratitude to Dr. Marvin Cheng for the continuous support and constructive criticisms of my Masters research; for his patience, enthusiasm, and encouragement in the advancement of this work. I am extremely grateful to Dr. Valeriya Gritsenko, whose research inspired me and guided this project. I also would also like to thank my fellow labmate in West Virginia University, Lei Jiang for his support and collaboration in the execution of this research; for the sleepless nights we were working together before deadlines; for all the lab devices that we broke and never told anybody; and for all the fun we have had in the last two years.

I would also like to thank the U.S. Immigration Service under the Obama administration, whose visa background security check frightened me to go back home during summer time and forced me to stay in the United States continuously and focus on my research. I would like to thank my very best friends Mohammad Fanaei, Sobhan Soleimani, and Amir Hussein Houshmandyar in particular; and all my friends in Morgantown, without them I would have graduated earlier.

Last but not the least, I would like to thank my family: my parents Fereshteh Eshaghi and Mahmoud Shisheie, for giving birth to me at the first place, countless nights taking care of me, and supporting me spiritually and financially throughout my life.

At the end, thanks to you, reader. If you are reading this line after the others, you at least read one page of my thesis. Thank You.

This thesis is dedicated to my family members for the love, support and encouragement provided by them. 


\section{Table of Contents}

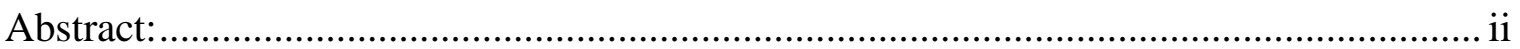

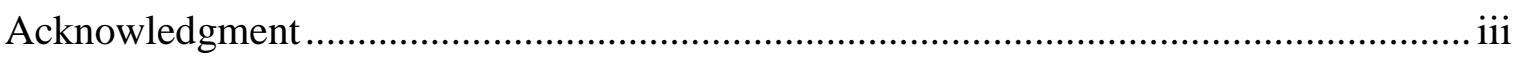

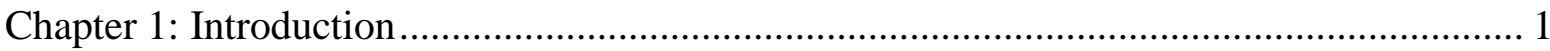

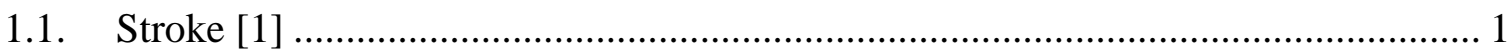

1.1.1. History of Stroke Neuro-Rehabilitation [12] ......................................... 2

1.2. Upper-Limb Exoskeleton Robotics and Applications in Assistance and

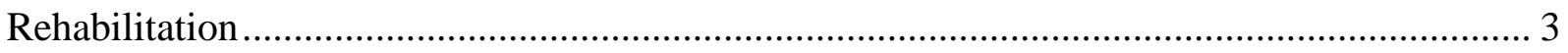

1.3. Motivation for Research ............................................................................. 5

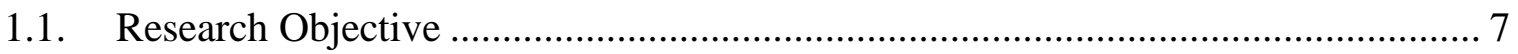

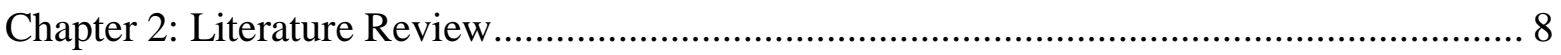

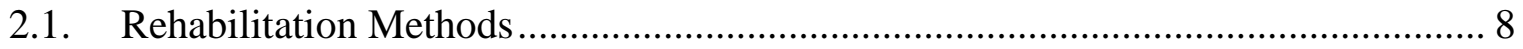

2.2. Home Rehabilitation Robotic Systems and Tele-Rehabilitation Applications ....... 10

2.3. Assessment and Evaluation Using Virtual Environment.................................. 12

2.4. Categories of Rehabilitation/Assistive Robots ................................................. 13

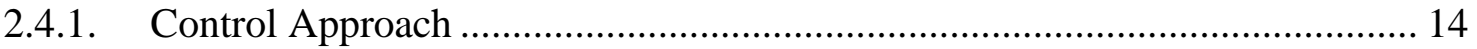

2.4.2. Therapeutic Approach and Physical Shapes............................................. 15

2.4.3. Actuation and Power Transmission Approach ........................................... 24

Chapter 3: Human Anatomy and Mechanical Design ................................................ 37

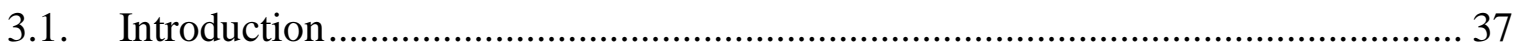

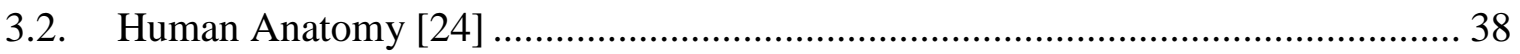

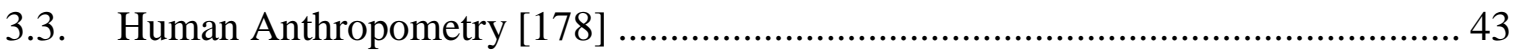

3.4. Exoskeleton Robot Design Criteria from the Anatomical View Point .................. 46

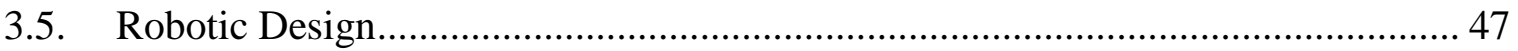

3.5.1. Targeted Users and Subsequent Anthropomorphic Data ............................ 48 
3.5.2. One-DOF Elbow Assistive Prototype............................................................ 51

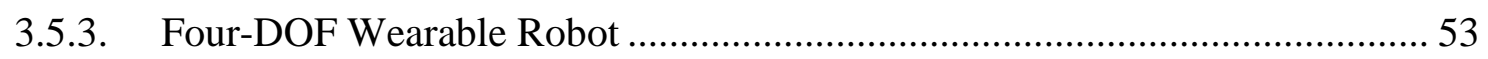

Chapter 4: Twisted String Actuation Design ......................................................................... 70

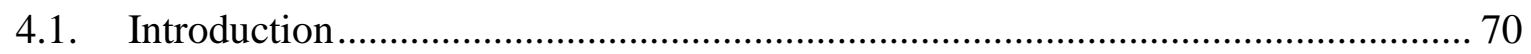

4.2. Twisted String Actuation Modeling [203] .......................................................... 71

4.3. Experiments with String Configurations and Types ............................................ 75

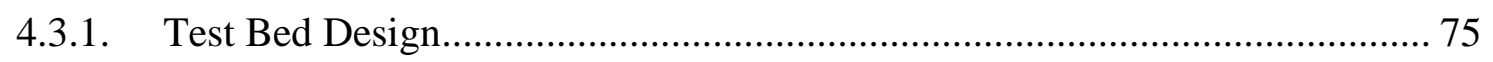

4.3.2. Twisted String Actuation Tests and Results.................................................... 80

4.3.3. Summary of Results and Analysis ................................................................. 106

4.4. Conclusion and Actuation Selection ...................................................................... 107

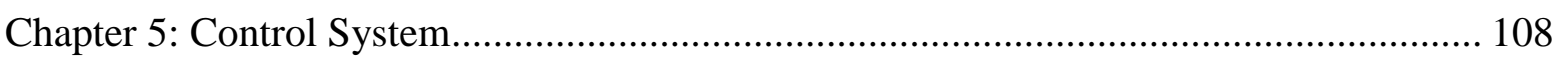

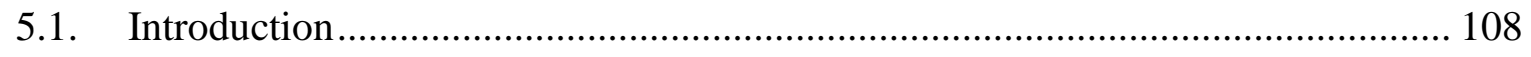

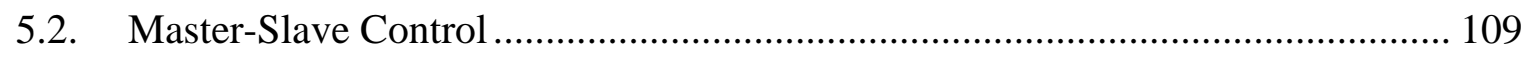

5.2.1. Master-Slave Control Architecture [216] [217] .......................................... 110

5.2.2. Controller Architecture, and Analysis ............................................................. 112

5.3. Component Models, Actuation Selection and Identification................................. 115

5.3.1. DC Motor Modeling and Identification [218] [219] ………….................... 115

5.3.2. Twister String Actuation Converter ………….......................................... 118

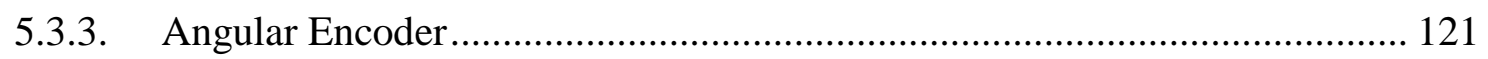

5.4. Test Bed Design and Experimental Procedures.................................................... 121

5.5. Root Locus Analysis, Gain Selection, and Analysis............................................. 124

5.6. ADL Trajectory, Control Performance, and Experimental Procedures................. 136

5.7. Torque Control and Estimation....................................................................... 139

Chapter 6: Conclusions and Future Works ..................................................................... 142

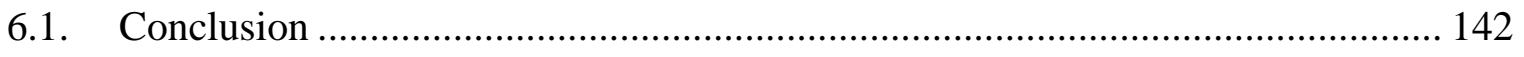




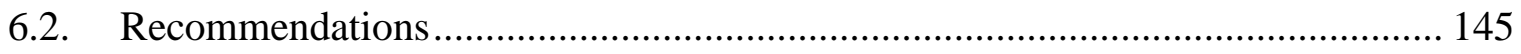

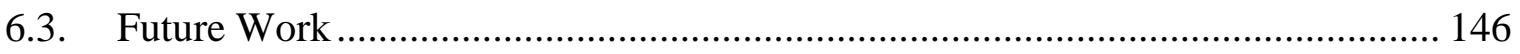

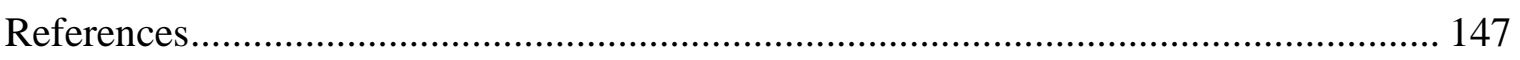

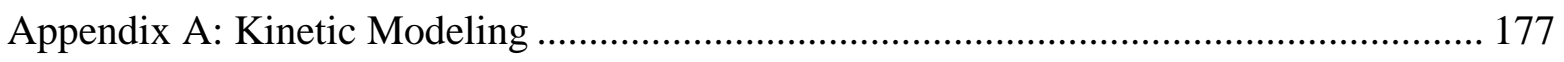

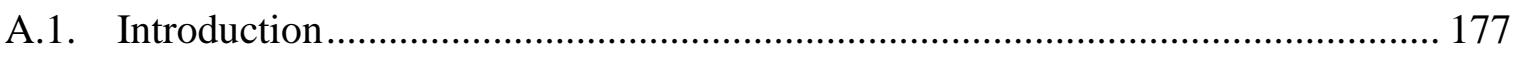

A.2. Euler-Lagrange Method and Modeling of the Upper Extremities ........................ 177

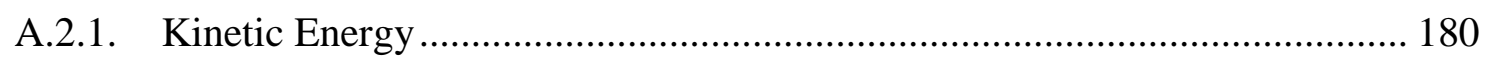

A.2.2. Potential Energy ……………………………....................................... 183

A.2.3. Rayleigh Dissipation Function ………………………………………..... 184

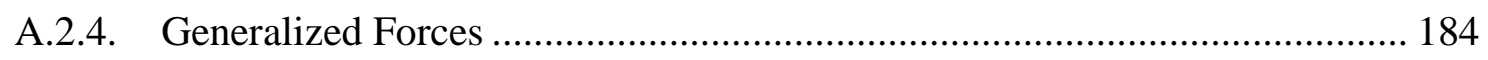

A.2.5. Equation of Motion Using Euler-Lagrange Method and RDF ...................... 184

A.3. Forming Equations of Motion........................................................................... 189

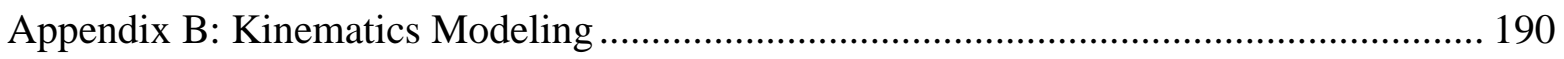

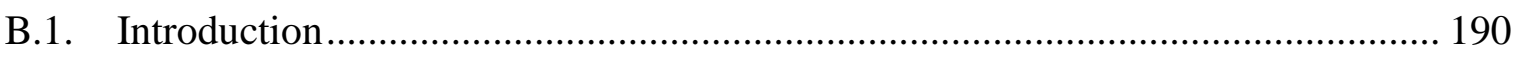

B.2. Kinematics of the Upper Extremities [222] ..................................................... 190

Appendix C: Technical Drawing ................................................................................. 199

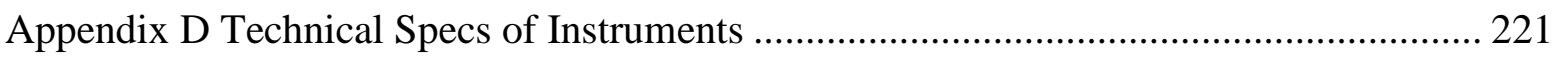

D.1. 19:1 Metal Gear DC motor 37Dx52L mm with 64 CPR Encoder [223] .............. 222

D.2. Angular Encoder HEDS-5500\#H06 ……………………………………..... 224

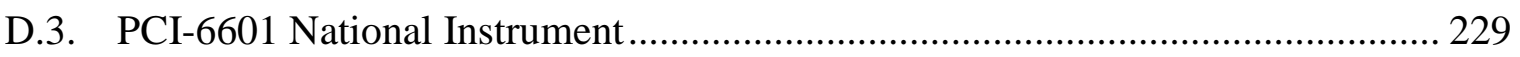

D.4. PCI-6221 National Instrument ........................................................................ 231 


\section{List of Figures}

Figure 2. 1. Categories of upper limb stroke rehabilitation methodologies and technologies 9

Figure 2. 2. (a) One degree of freedom (DoF) robot device for wrist rehabilitation. (b) Two DoF robot device for elbow-shoulder rehabilitation [61] .................................................. 13

Figure 2. 3. Categories of Rehabilitation Robots ......................................................... 13

Figure 2. 4. Rehabilitation Robots Classification from the Point of View of Control and

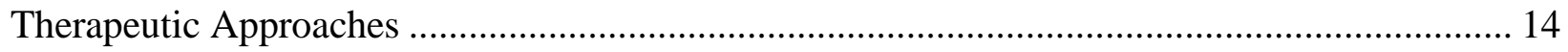

Figure 2. 5. Upper limb rehabilitation robot mechanical categories [69]......................... 16

Figure 2. 6. Stroke-patient during therapy at the Burke Rehabilitation Hospital (White Plains, NY). Therapy is being conducted with a commercial version of MIT-MANUS (Interactive Motion

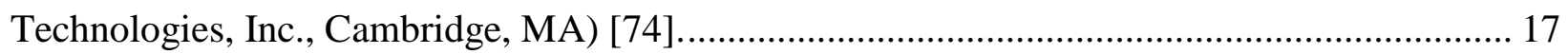

Figure 2. 7. MIME robot while delivering unilateral therapy [81] ................................. 19

Figure 2. 8. Computer-assisted arm trainer; patient with left hemiparesis practices a repetitive bilateral pronation and supination movement of the forearm [71] ........................................ 20

Figure 2. 9. (Left) ARMin Mechanical structure, sensors and motors, (right) ARMin with four

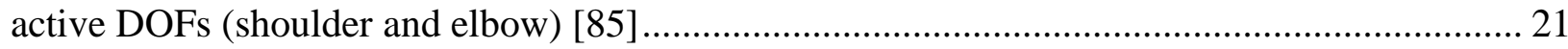

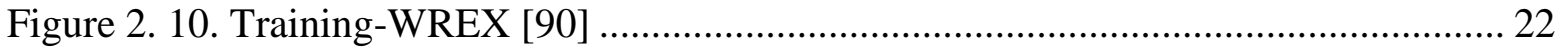

Figure 2. 11. The patient's right forearm is fastened into the splint to receive sensorimotor stimulation with NeReBot at the bedside position (left) in the sitting position (right). [90] ........ 23

Figure 2. 12. (Left) Electric motors and cable drives arrangements at the shoulder mechanism

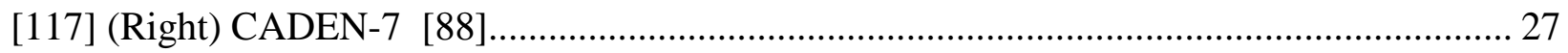

Figure 2. 13. (Left) MEDARM system consists of a 6DOF robotic exoskeleton mounted onto a support structure. (Right) A CAD drawing of the MEDARM mechanism showing the orientation of joint, cables and symbolic limbs masses. 28

Figure 2. 14. ARMin II robot and a patient sitting on it. A physiotherapist is sitting nearby to select the rehabilitation procedure [86]. 28

Figure 2. 15. Lightweight exoskeleton that allows basic pinching motion [123]............... 29

Figure 2. 16. Wege's finger exoskeleton CAD drawing. Two Bowden cables are used for each

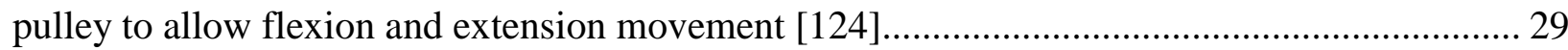

Figure 2. 17. Finger prototype actuated by the twisted string actuation system. [125] [126] 30 
Figure 2. 18. Structure of the prototype of the finger robot designed by Sonada and his group [129] [130] [131] 31

Figure 2. 19. Finger robot developed by Aukes while joints 2 and 3 are locked and holding

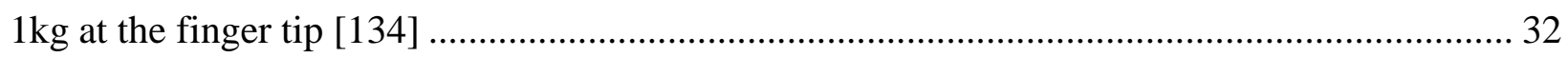

Figure 2. 20. Developed dual-mode robot finger by Shin [135] ..................................... 33

Figure 3. 1. Different shapes of McKibben Muscles [103] ........................................... 25

Figure 3. 2. The schematic demonstration of the upper extremities and thorax [24] [156] .. 38

Figure 3. 3. Seven major degrees of freedom of the upper-extremities [158].................... 39

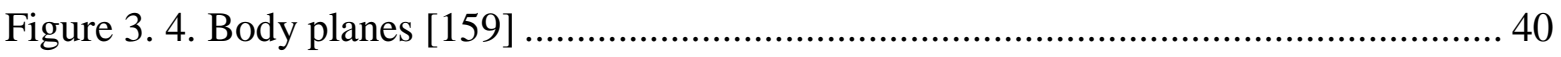

Figure 3. 5. The components of the shoulder joint [162] [163] .................................... 40

Figure 3. 6. References of shoulder motion, (a) shoulder flexion/extension, (b) shoulder abduction/adduction, and (c) shoulder rotation [164] [165]

Figure 3. 7. (a) Elbow complex, (b) hinged joint, and (c) motion provided by the elbow

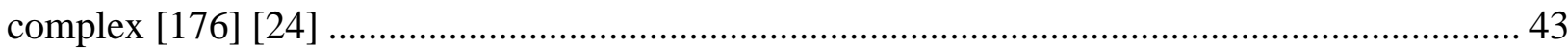

Figure 3. 8. Segments length as a function of height [178] ...................................... 44

Figure 3. 9. Critical Dimensions by Height Range ................................................. 50

Figure 3. 10. (a) Solidworks model of the structure of the robot (b) Full flexion mechanical

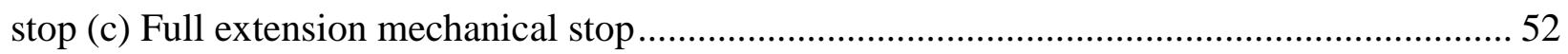

Figure 3. 11. Prototype mounted on the upper extremities in different positions ............... 53

Figure 3. 12. Schematic of the upper extremities with driving torques............................ 54

Figure 3. 13. Schematic of the upper extrmity in maximum required torque position.......... 56

Figure 3. 14. Solidworks model of the robotic hand .................................................... 57

Figure 3. 15. Direction of the actuating cables .......................................................... 58

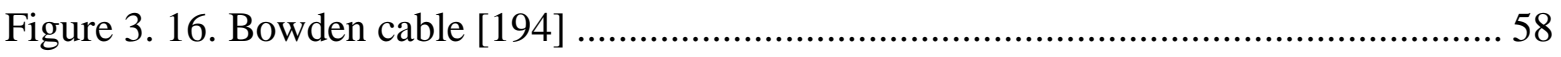

Figure 3. 17. Range of motion for flexion and extension of shoulder and elbow joints ....... 58

Figure 3. 18. (Top) Schematic of the arm holders and (bottom) schematic of the arm robot on

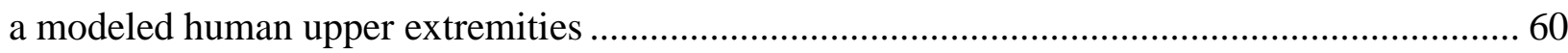

Figure 3. 19. Backpack design and how it is set on human......................................... 61

Figure 3.20: (Left) three axes of rotation in non-singular condition (right) in gimbal lock [197] 
Figure 3. 21. Two rapid prototypes of the shoulder rehabilitation exoskeleton with (left) singular combination of joints and (left) non-singular combination. Photos reproduced from reference [158].

Figure 3. 22. Representations of angles at the elbow and shoulder joints in accordance with recommendations of International Society of Biomechanics (ISB): (a) elbow flexion ( $\alpha$ ), (b)

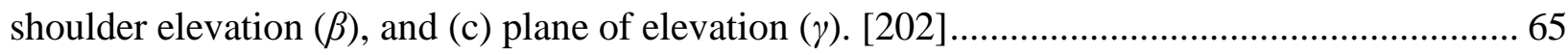

Figure 3. 23. Five links of the shoulder complex from (a) top view (b) back view ............. 65

Figure 3. 24. The position of limbs when $\psi_{1}=0^{\circ}, \psi_{2}=0^{\circ}, \psi_{3}=0^{\circ}$, and $\psi_{4}=0^{\circ}$, where the upper arm and forearm are are standing parallel to the Sagittal and Frontal planes

Figure 3. 25. The position of limbs when $\psi_{1}=10^{\circ}, \psi_{2}=0^{\circ}, \psi_{3}=0^{\circ}$, and $\psi_{4}=0^{\circ}$, where the upper arm and forearm are are only parallel to the Frontal plane.

Figure 3. 26. The position of limbs when $\psi_{1}=60^{\circ}, \psi_{2}=0^{\circ}, \psi_{3}=0^{\circ}$, and $\psi_{4}=0^{\circ}$, where the upper arm and forearm are are only parallel to the Frontal plane, and $\psi_{1}$ has the maximum angle 68

Figure 3. 27. The position of limbs when $\psi_{1}=10^{\circ}, \psi_{2}=0^{\circ}, \psi_{3}=30^{\circ}$, and $\psi_{4}=45^{\circ}$, where the model is approaching the omaginary cup of coffee to grab

Figure 3. 28. The position of limbs when $\psi_{1}=10^{\circ}, \psi_{2}=60^{\circ}, \psi_{3}=0^{\circ}$, and $\psi_{4}=90^{\circ}$, where the model is approaching mouth to drink coffee

Figure 3. 29. The position of limbs when $\psi_{1}=28^{\circ}, \psi_{2}=55^{\circ}, \psi_{3}=20^{\circ}$, and $\psi_{4}=130^{\circ}$, where the model is drinking coffee from the imaginary cup of coffee 69

Figure 4. 1. Twisted string configuration of a two-string actuation ((a) and (b)) and threestring actuation ((c) and (d)) with and without a neutral strand [203]. 72

Figure 4. 2. (a) Schematic representation of twisted string and forces (b) Equilibrium of acting

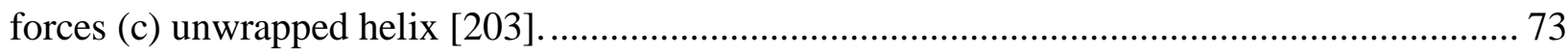

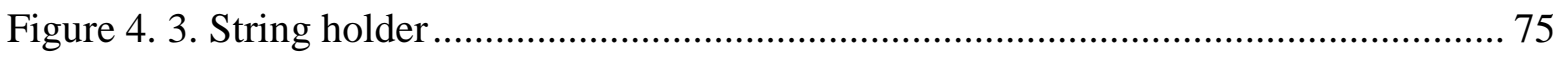

Figure 4. 4. (a) Schematic of test stand (b) actual test stand ......................................... 76

Figure 4. 5. Model of the slider with constant velocity .............................................. 77

Figure 4. 6. Axial force for upward and downward motion ....................................... 78

Figure 4. 7. Different configurations of the strings used in the experiment ....................... 81 
Figure 4. 8. Comparison of the theoretical transmission length and experimental measurement for $100 \mathrm{~cm}$ length 82

Figure 4. 9. Repetitive tests of the twisted actuation system with different transmission length

Figure 4. 10. $\Delta \mathrm{p}$ of contraction and expansion for (a) $100 \mathrm{~cm}$, and (b) $50 \mathrm{~cm}$ Hysteresis of contraction relative to expansion for (c) $100 \mathrm{~cm}$, and (c) $50 \mathrm{~cm}$ 84

Figure 4. 11. Two-string actuation with and without a neutral string .... 86

Figure 4. 12. Oscillatory motion of two-string actuation with and without a neutral string 87

Figure 4. 13. Three-string actuation with a without a neutral string ..... 89

Figure 4. 14. Four, five, and six-string actuation with a neutral string ............................ 90

Figure 4. 15. Six-string actuation with a without a neutral string 91

Figure 4. 16. The responses of the actuation system under $3500 \mathrm{~g}$ load with different stringconfigurations 93

Figure 4. 17. (a) Worn out part of the sting (b) string after use (c) string before use .......... 94

Figure 4. 18. Two-string actuation with a without a neutral string ................................. 95

Figure 4. 19. Three-string actuation with a without a neutral string ............................... 97

Figure 4. 20. Four, five, and six-string actuation with a neutral string ............................ 98

Figure 4. 21. Six-string actuation with a without a neutral string …................................ 99

Figure 4. 22. The response of the actuation system under $3500 \mathrm{~g}$ mass load with different string-configurations 101

Figure 4. 23. (a) Worn out part of the sting (b) string after use (c) string before use ......... 101

Figure 4. 24. The response of a 2-string actuation in linear and nonlinear range 103

Figure 4. 25. Geormetry of (a) the string holder at maximum contraction without going to nonlinear area (b) 2-string actuation at maximum contraction (c) and (d) (b) 2-string actuation at nonlinear area (kinking mode) 104

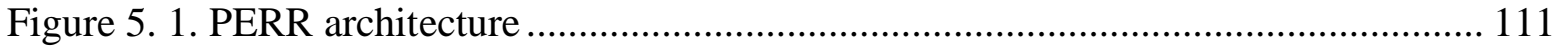

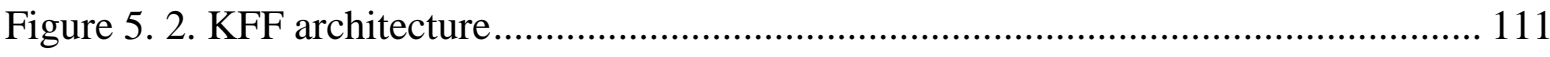

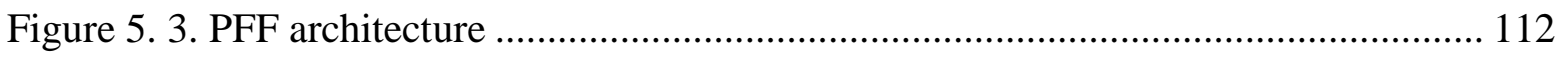

Figure 5. 4. Schematic of the master-slave control ..................................................... 113

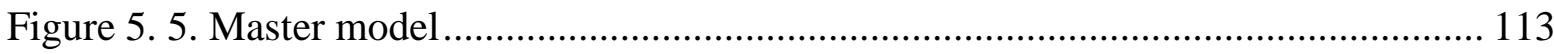




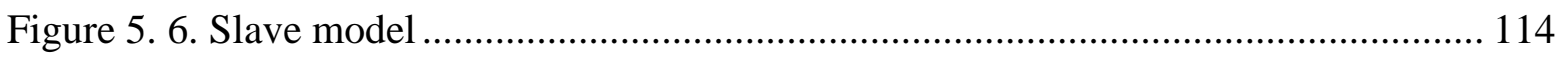

Figure 5. 7. Input and outputs of the system using Chirp signal ...................................... 116

Figure 5. 8. Input and output of the approximated transfer function................................... 117

Figure 5. 9. Behavior of the actuator with 6 active strings and one neutral string ............. 119

Figure 5. 10. Conversion from contraction to angle ............................................................ 119

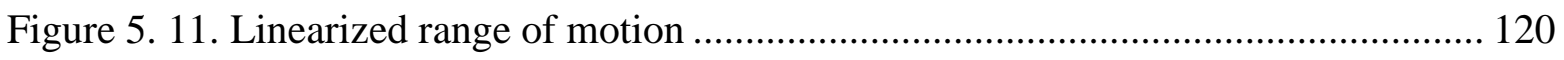

Figure 5. 12. Schematic of the actuation acting on the robot arm ...................................... 122

Figure 5. 13. Schematic of the test bed when (a) the left actuator contracts and the right actuator releases, (b) only the right actuator releases (error due to conflict is marked in green) 122

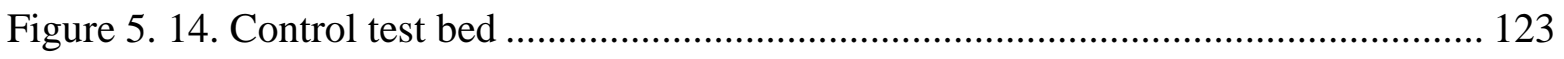

Figure 5. 15. Skematic of the twisted string actuation once mounted on the test stand ...... 124

Figure 5. 16. Root locus of the master with a gain controller ............................................. 127

Figure 5. 17. Root Locus of master with PID controller ................................................... 128

Figure 5. 18. Root Locus of the slave with a gain ......................................................... 129

Figure 5. 19. Root locus of the slave with PID.............................................................. 129

Figure 5. 20. Response of master to unit disturbance ......................................................... 132

Figure 5. 21. Response of slave to unit disturbance ........................................................ 133

Figure 5. 22. The architecture of the synchronization control............................................. 134

Figure 5. 23. Trajectory performance of the master-slave synchronization control without

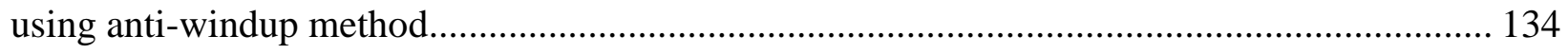

Figure 5. 24. Trajectory performance of the master-slave synchronization control using anti-

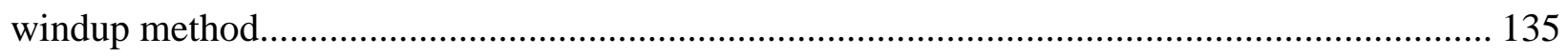

Figure 5. 25. Upper extremities motion at the test to drink water [221] ........................... 136

Figure 5. 26. Elbow joint angle while performing targeted ADL ….................................. 136

Figure 5. 27. Angle trajectory of the simulation and experiment ..................................... 137

Figure 5. 28. Tracking error of the simulation and experiment.......................................... 138

Figure 5. 29. Contraction error …………...................................................................... 139

Figure 5. 30. Flowchart of using appendix A and B to compute the required current ........ 140

Figure A. 1. Schematic of the upper extremities ......................................................... 179 
Figure B. 1. Position and orientation of body-fixed reference frame relative to global reference

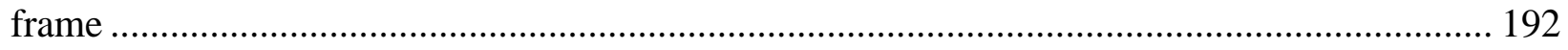

Figure B. 2. Generalized coordinates of the robotic system ........................................ 195

Figure D. 1. 19:1 Metal Gear DC motor 37Dx52L mm with 64 CPR Encoder ................. 222 


\section{List of Tables}

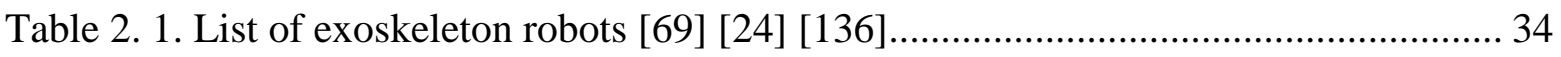

Table 3. 1. Weight, center of gravity, length, and radius of gyration of targeted limbs of upper

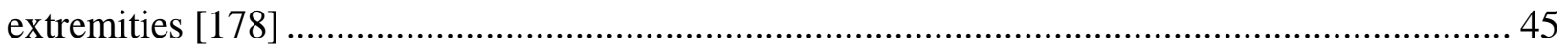

Table 3. 2. Weigh $(\mathrm{kg})$ of the targeted age and percentile [192] ..................................... 48

Table 3. 3. Height (centimeter) of the targeted age and percentile [192] ........................... 49

Table 3. 4. Critical Dimensions by Height Range ........................................................ 49

Table 3. 5. Range of weight and moment of inertia of the limbs of the target range ........... 50

Table 3. 6. Physical properties of the $20 \%$ model ....................................................... 51

Table 3. 7. Parameters of upper extremities ................................................................... 55

Table 3. 8. Static forces needed to maintain the arm stationary .................................... 56

Table 3. 9. Shoulder and elbow axial contraction ......................................................... 59

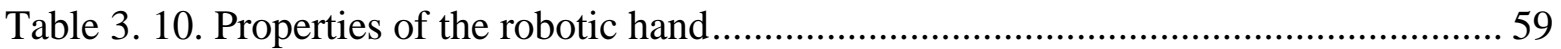

Table 3. 11. Range of motion of shoulder and elbow joints according to the ISB standard

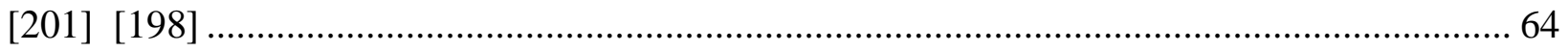

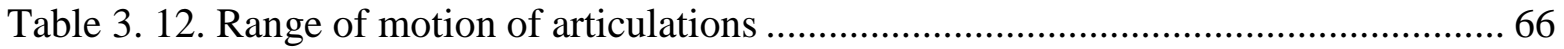

Table 4. 1. Friction of the slider in stage one and two and in two directions ...................... 78

Table 4. 2. Characteristics of different configurations of the utility cable strings .............. 93

Table 4. 3. Characteristics of different configurations of the tactical cable strings ........... 100

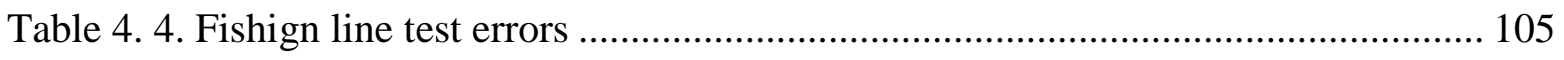

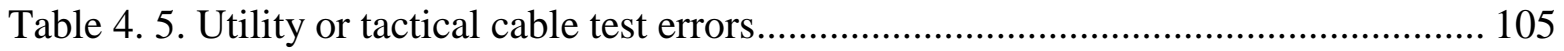

Table 4. 6. Test errors ............................................................................................. 105

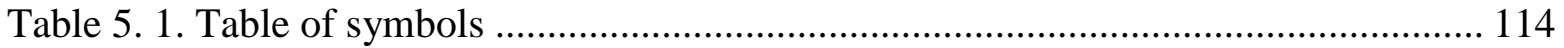

Table 5. 2. Values of the transfer function parameters ............................................... 117

Table 5. 3. Gains of the PID controller of the master ................................................ 128

Table 5. 4. Gains of the PID controller of the slave .................................................... 130

Table 5. 5. Gains used for the synchronization control ............................................... 135 
Table D. 1. DC Motor Dimensions ..................................................................................... 222

Table D. 2. General specifications ..................................................................... 223 


\section{Chapter 1: Introduction}

\subsection{Stroke [1]}

Stroke, or cerebrovascular accident (CVA), is one of the major causes of physical impairment in which the brain loses its functionality due to a disturbance in blood supply, and can cause permanent neurological damage, complications, and death. As a result, the affected area of the brain does not function properly anymore, which might result in an inability to move one or more limbs on one side of the body as well as difficulties in cognition and sensing, inability to understand or formulate speech, or an inability to see one side of the visual field [2].

Stroke could soon be the most common cause of death and impairment worldwide [3]. Stroke is the primary cause of permanent impairments and neurological damage in the United States and Europe [4] [5] and it is currently the second leading cause of death in the western world, ranking after heart disease and before cancer. After Ischemic heart disease with 6.26 million deaths [2], 
stroke caused the death of 4.38 million people (almost 3 million in developing countries), and causes $10 \%$ of deaths worldwide [6]. Annually, about fifteen million individuals worldwide suffer from stroke, which kills about one third of them [7]. Moreover, the likelihood of getting stroke increases exponentially from the age of 30 , and the distribution varies etiologically and by age [8]. Advanced age is one of the most significant stroke risk factors. 95\% of strokes occur in people at age 45 and older, and two-thirds of strokes occur in those over the age of 65 [9] [10]. However, stroke can occur at any age, including in childhood.

Treatment to recover any lost function due to stroke is termed stroke rehabilitation. The primary goals of stroke management are to reduce brain injury and achieve maximum patient recovery. An acute stroke units are specialized in providing medical and surgical care aimed at stabilizing the patient's medical status [11]. Once a patient is medically stable, the focus of their recovery shifts to rehabilitation.

\subsubsection{History of Stroke Neuro-Rehabilitation [12]}

Knowledge of stroke and the post-stroke recovery process have developed enormously in the late 20th century and early 21 st century. Johan Wepfer, a Swiss pathologist and pharmacologist, was the researcher who proposed the first theory about the causes of stroke. By studying the brain of a pig in 1620 , he came up with the theory that stroke was caused by an interruption in blood supply to the brain [13]. This discovery was the most important milestone at that time. However, from this discovery emerges the question of how to treat patients with stoke and cure them.

For many years, it was believed that being active after stroke is not beneficial and it results in deterioration of the patients' health condition. This attitude changed around the 1950s when health professionals observed physical recovery of those patients who benefited from therapeutic 
exercises. The outcome was so promising that being able to transfer from the bed to the wheelchair without assistance was considered a level of accomplishment. Even though such achievements seem inconsequential, the situation was improving.

Twitchell focused his studies on the recovery patterns of patients having stroke in the early 1950s. His observations indicated that there is a $70 \%$ chance of making a full or good recovery, if there is some recovery of hand function in the first four weeks of stroke. According to his report, major recovery can be achieved in the first three months and only minor recovery occurs after six months [14]. His report demonstrated the importance of rehabilitation and employing clinical methods to recover the ability, which is affected by stroke.

More recent research has demonstrated that many years after stroke, significant improvement is not out of reach. It is nowadays known that the human brain has more capabilities to adapt itself to the new situation after stroke and to recover control of limbs than once believed. Brain imaging shows that the motor cortical part of the brain shrinks due to inactivity after injury but it can expand by subsequent exercise and activities [15] [16].

\subsection{Upper-Limb Exoskeleton Robotics and Applications in Assistance and Rehabilitation}

Research studies on both animals and humans have indicated that the recovery of functional skills cannot be fully achieved without the assistance of systematically-forced therapies. However, health insurers often deny rehabilitation to stroke survivors claiming patients have reached a plateau and the disease has become chronic several months after the stroke [17]. Moreover, economic pressures, aging of the population, and lack of specialists and available human resources can interrupt therapy, which impedes full recovery of patients after being discharged from hospital 
following initial rehabilitation [18]. Robotic devices, in particular movable robots, that provide rehabilitation therapy at home and clinics are a novel way not only to optimize the cost of therapy but also to let more patients benefit from rehabilitation for a longer time. It is commonly assumed that rehabilitation robotics is only used to assist disabled people [19]. However, in recent year the definition has been extended by Hillman to "the application of robotic technology to the rehabilitation needs of people with disabilities as well as the growing elderly population" [20].

In addition, patients whose level of mobility has reached a plateau need to be encouraged to use the disabled limb in order to keep it active [21] [22]. Assistive robots can be used to compensate the physical inability, which helps patients regain the lost power of the affected limbs. Exoskeleton robotics, both active and passive [23] is a solution for both rehabilitation and assistive purposes, which can be portable and convenient to use. Major applications of exoskeleton robots are in human power augmentation, robotic rehabilitation, human power assist, and haptic interaction in virtual reality [24].

Active exoskeleton robots were studied in the 1960s and 1970s [25] [26] [27] [28] for military, medical, and industrial applications. In the early 1990s, however, new applications for exoskeleton robots were proposed to amplify the strength of the human user [29] [30]. Unlike many other types of robots used in industry, special consideration must be taken in the design process of medical robots since these machines interact directly with human users. Adjustable work envelopes, safety, comfort, low inertia, and adaptability are the major items, which must be considered specifically in the mechanical design of such systems. Controllability, responsiveness, flexible and smooth motion, and safety should be considered in the controller design. Noting that the upper extremities has 7 major degrees of freedom (DOF), most of the current or proposed robots have fewer than seven degrees of freedom. Moreover, many recent upper-limb exoskeleton robots used serial 
manipulators with either electric motors or pneumatic muscles as the actuators. The active upperlimb exoskeleton robots can be classified according to the following items:

1. The actual segments of the upper-limb (hand exoskeleton robot, forearm exoskeleton robot, upper-arm exoskeleton robot or combined segments exoskeleton robot );

2. The number of DOF;

3. The actuators type (electric motors, pneumatic muscles, pneumatic piston, hydraulic actuators, a combination of them or other types);

4. The power transmission method (gear drive, cable drive, linkage mechanism, pneumatic or other);

5. The application of the robot (rehabilitation robots, assistive robots, human amplifier, combined use);

6. The type of the support or the mobility of the robot (hinged to a fixed frame like a wheelchair or a wall or a mobile frame or other).

\subsection{Motivation for Research}

Recently, research on home rehabilitation robotics and power assist technology for daily activities has increased, particularly in societies in which the number of physically weak (aged, injured and/or handicapped) individuals is increasing. Robots used for such purposes should be smaller, lighter and more affordable than the current enormously bulky, heavy and expensive robots used in clinics and hospitals. However, the same design criteria of other rehabilitation/assistive robots such as motion singularities in complex joints, dealing with the human motion of different people with different age and size, space limitation, weight-to-force ratio, agility in motion, and etc. should be taken into consideration. New technologies facilitate designing new devices, which are 
cheaper and have better performance. Moreover, there are certain areas of assistive technology that have not been touched so far such as affordable assistive robots. Such robots are not only wearable and extremely small but also provide just enough assistive force to assist the patient perform certain tasks. This assistive force is very little, and may not be more than $20 \%$ of the total required effort. Most assistive robots compensate for the entire loss ability or they are designed to provide power amplification to carry heavy loads. However, in some case, such as this research, just a small amount of force is needed to assist victims to pick up a cup or to take a book off the book shelf or to comb their hair. Since there is no necessity to provide big compensatory force, robot can be much smaller, cheaper and lighter. In addition, a wearable assistive robot, which provides a small assistive force encourages the victim to keep using the affected limb, which gradually improves motor skill and does contribution to rehabilitation process. Recent researches demonstrate that intense rehabilitation can increase the pace of improvement [31] [32]. Thus, the outcome of bring rehabilitation into homes is not only decreasing the cost of rehabilitation compared to treatment in a clinic, but also improving the pace of rehabilitation, which means that victims can gain the loss ability faster than ordinary methods. Moreover, bringing victims back to ordinary life makes them active and profitable again too.

A new design for the exoskeleton assistive/rehabilitation robot in the current research should be such that it overcomes one or some of the following obstacles in this area:

1. It should be able to provide the desired compensation for the affected limb.

2. The motion should be natural and smooth and must not interfere with normal human motion.

3. It should be capable of being used for some major exercises needed for rehabilitation or some Activities of Daily Living (ADL).

4. It should be wearable and mobile for daily activities or home rehabilitation. 
5. It should be lighter and smaller than similar models available on market.

6. It should be affordable.

\subsection{Research Objective}

The current research plan is to design a rehabilitation/assistive robot for daily activities. The robot will be used for the upper extremities. To decrease weight and size, new actuation technologies will be employed. Thus, to simplify the design process for the first phase, the motion is limited to the elbow joint. The objectives of this research are:

1. To determine several ADLs of the upper extremities such as picking up a glass of water or taking a book off a book shelf and targeting one for controller design. It is necessary to determine the trajectory of the limbs while performing ADLs.

2. To design a one-DOF assistive robot for the elbow joint and eventually a four-DOF robot for shoulder and elbow. Novel actuation technologies must be used such that the robot is small, light, and cheap. The elbow robot should be wearable and able to compensate for up to $20 \%$ of the required force to perform flexion and extension.

3. To use a proper model or to provide a dynamic model of the upper extremities and to design a proper controller such that it tracks the identified trajectory of the limb.

The scope of this work thus consists of the development of an active exoskeleton robot for the elbow joint and possibly the shoulder joint, which assists patients to regain their strength and mobility by providing a small amount of force assistance. The first prototype has one degree of freedom and it provides a springboard for the next generation with four DOF. 


\section{Chapter 2: Literature Review}

In this chapter current rehabilitation methods were skimmed firstly. Then rehabilitation robots were categorized and among all of them exoskeleton robots were selected and different aspects of this field were discussed.

\subsection{Rehabilitation Methods}

Available upper limb stroke rehabilitation methodologies and technologies can be categorized as: conventional physical and occupational therapy, constraint-induced movement therapy, roboticaided and sensor-based therapy systems [33]. 


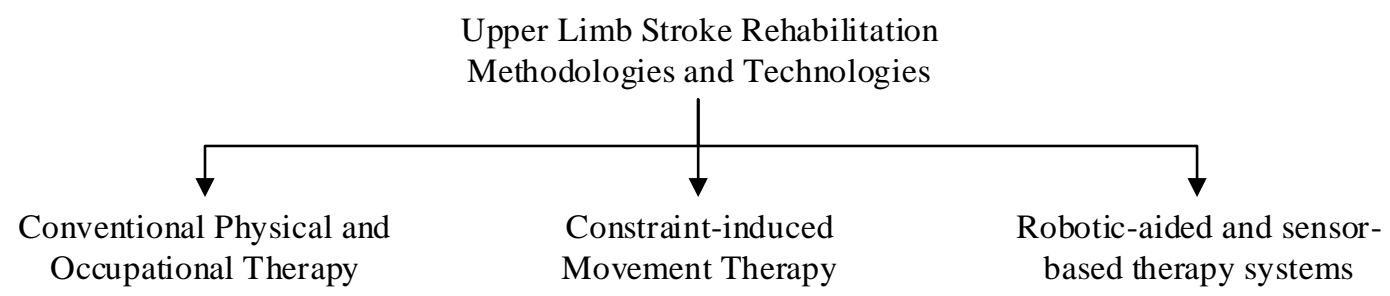

Figure 2. 1. Categories of upper limb stroke rehabilitation methodologies and technologies

Physical therapy (PT) for most stroke patients is the start point and the basis of the rehabilitation process [34]. The therapist uses certain activities such as training, exercises, and physical manipulation of the stroke patient's body in order to restore the loss function and help the patient regain balance. Since the patient has partially or fully lost the ability to perform simple tasks, exercises are planned such that the patient relearns simple activities such as walking, sitting, standing, lying down, and the process of switching from one type of movement to another. In occupational therapy (OT), however, the exercises are designed such that the patient regains independence such that he/she is able to perform everyday tasks such as eating, drinking, dressing, bathing, cooking, reading and writing, and toileting [34].

Constraint-induced movement therapy (CI or CIMT) is a category of rehabilitation therapy in which motor control of the limbs and the damage to the nervous system are improved by forcing the patient to use the affected limb [35].

Robot-aided therapy is involved in rehabilitating stroke patients using robots, which are used mainly as therapy aids instead of assistive devices [36]. Rehabilitation robotics includes development of robotic devices, developing schemes of therapeutic training [37], and assessing the performance of the patient [38].

In this chapter, the focus will be specifically on robotic-aided therapies and sensor-based therapy systems and on the related applications of exoskeleton robots in this field of rehabilitation. 
Detailed systematic technical reviews, comparison of physiotherapy schools, effects of intensity of certain training, and efficacy of specific upper limb rehabilitation techniques are beyond the scope of the present research [39] [40] [41] [42] [43] [44]. Auxiliary applications such as virtual reality applications, auditory feedbacks and etc. are often integrated with the exoskeleton robot to increase the efficacy of therapy [45], which is also beyond the scope of this research.

\subsection{Home Rehabilitation Robotic Systems and Tele-Rehabilitation Applications}

Using home rehabilitation following clinical rehabilitation has received substantial interest recently. About 50\% of stroke survivors upon discharge from hospital and after receiving inpatient (acute) rehabilitation still experience upper arm disabilities [46]. The importance of home rehabilitation using challenging and interactive games/software unfolded once researchers found out the negative impacts of Learned Non-Use (LNU). LNU undoes the rehabilitation progression in under-supervised environments outside the hospital. LNU, which affects most stroke survivors [47], is a common involuntary reflex of the brain to gradually use the less-impaired limbs despite existing functional gains in the more impaired limb in order to accomplish tasks [48]. In order to maintain the more-impaired limb improvement, it is necessary to avoid compensatory behaviors such as LNU [46].

The challenges of designing rehabilitation robots and using them outside the clinical environment are not limited to LNU. Remaining factors include, but are not limited to the following: 1) availability of the therapist, 2) cost of long-term rehabilitation, 3) convenience of using robots - including their ability to be used easily and quickly - and robot adaptability to variant therapies [49]. To have an effective home rehabilitation, the patient should be visited by 
the therapist on a regular basis to ensure improvement [50] [51] [52]. These visits can be done through tele-rehabilitation, which not only reduces the costs, but also facilitates the communication of the patient with doctors [53].

Tele-rehabilitation can be achieved by means of tele-consultation using video-conferencing, tele-monitoring technologies used to monitor and assess patients' performance. Tele-therapy allows therapists to change the gains and test different therapies while the patient is using the robot, and tele-cooperation allows multiple patient cooperation to complete a task.

Tele-rehabilitation technologies facilitate communication between patients and doctors and cut the costs substantially by bringing robots from clinics to homes. Designing new affordable robots for personal use is a new challenge. An affordable system cannot be achieved unless it is lighter, smaller, and more portable than current models and it uses simple games and provides feedback to therapists by tele-communication. In addition, since the patient is in the sub-acute phase, a complex robot is not needed anymore. Using passive joints without actuation or designing the system such that it has fewer degrees-of-freedom in comparison with complex and bulky clinical robots are the alternatives.

One of the first applications for tele-rehabilitation is the Java Therapy system, which is an end-effector robot [54]. The Java Therapy system is an affordable robotic system, which delivers unilateral therapy focusing on forearm pronation/supination and wrist flexion/extension. It uses a library, which is embedded in a website to evaluate the improvements of the patients. The feedbacks are sent by a commercial force joystick, which allows therapist to change the mode (resistive, assistive, and etc) while the user is playing with. In addition, it allows the occupational therapist guide the patient online and evaluate the progress based on feedbacks. The website, however, can assess the movement performance through web too [54]. Rutgers Master II [55], is 
another example of exoskeleton robot, which benefits from remote therapy. To deliver effective home therapy of hand rehabilitation, Rutgers Master II lets the therapist modify the relevant gains remotely.

\subsection{Assessment and Evaluation Using Virtual Environment}

The new challenge facing home-based rehabilitation robots is providing safe and reliable virtual environment during unsupervised therapy. Therapist availability is one of the contributing factors of effective tele-rehabilitation, which not only keeps the cost of rehabilitation high, but also limits the time of each session to availability of therapists. New games provide an offline environment in which the patient plays and the results are sent to a therapist to be evaluated. Offline games can motivate patients' participation in rehabilitation process too.

Palanca is a home-based therapy environment developed by Bach-y-Rita and colleagues [56] for Computer-Assisted Motivating Rehabilitation (CAMR). It is used for quantifying impairment by means of a mechatronized handle and a computerized game of pong. Johnson and colleagues [57] used a low-cost joystick (TheraJoy) to deliver therapy in vertical and horizontal planes. They proposed a Robot/Computer-Assisted Motivating Rehabilitation, which uses various low-cost joystick and wheels such as TheraDrive [58]. TheraJoy benefit a unified custom-designed software called UniTherapy to deliver therapy [59]. Colombo and colleagues developed two robots under one package [60]. The package consists of a 1-DOF robot for wrist and a 2-DOF shoulder/elbow robot. The focus in this work was on designing a package, which increases patient's motivation to participate in process actively. To achieve this task, they developed motivating software such as a simulated piano that presents visual, auditory and tactile feedback to enable bilateral arm and hand therapy using cyberglove [61]. 

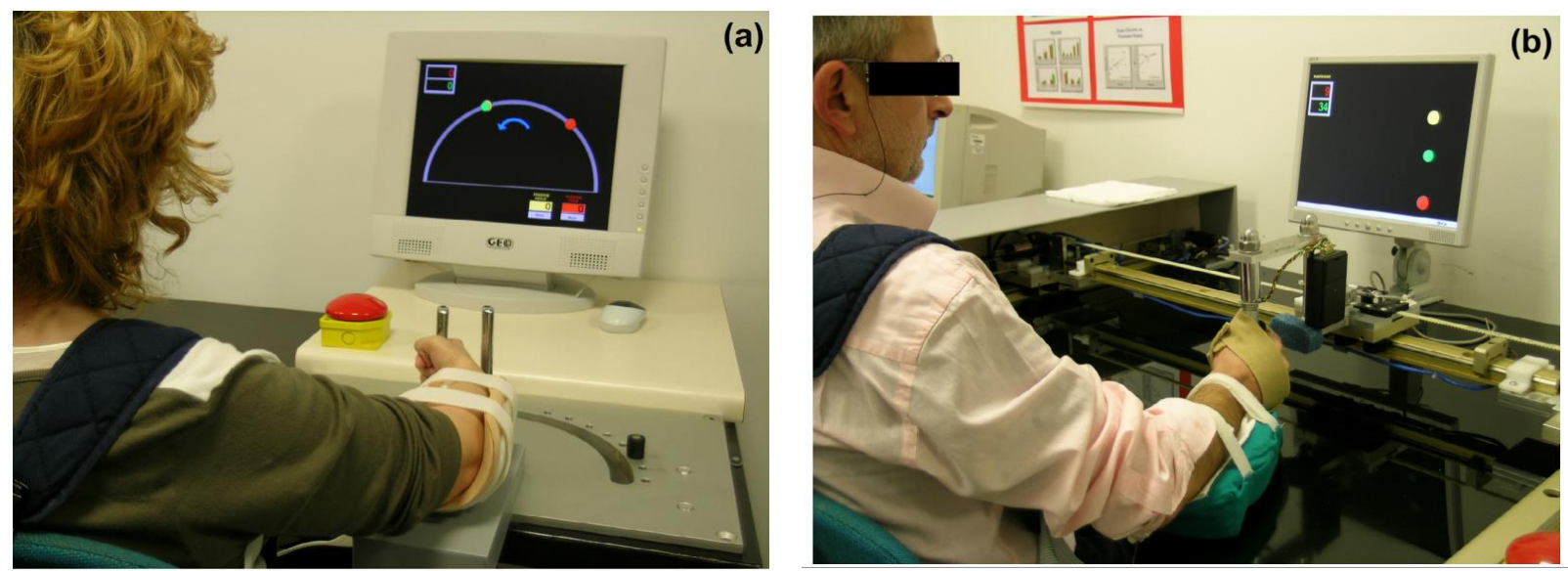

Figure 2. 2. (a) One degree of freedom (DoF) robot device for wrist rehabilitation. (b) Two DoF robot device for elbow-shoulder rehabilitation [61]

\subsection{Categories of Rehabilitation/Assistive Robots}

To target the right rehabilitation approach, it is crucial to explore different types of rehabilitation robots, their applications, and pros and cons.

In this section various rehabilitation robots are categorized into subcategories from different perspectives and their applications are discussed. The categories will be from different views such as control, physical shape, and etc. Categories of the rehabilitation robots are shown in Figure 2. 3.

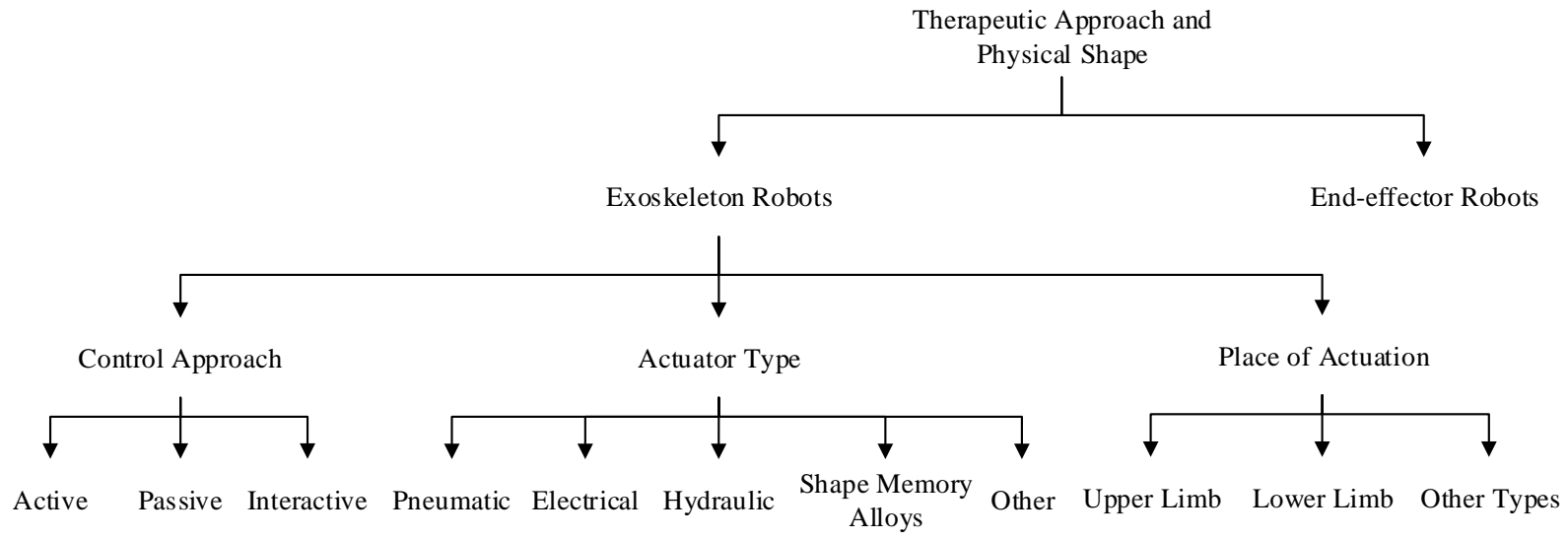

Figure 2. 3. Categories of Rehabilitation Robots 


\subsubsection{Control Approach}

Rehabilitation robots from the point of view of control can be categorized as passive, where the robot constraints the patient's limb to a predetermined range of motion without actuation; active, where the robot leads the patient's limbs on a predefined trajectory or toward a point using a type actuation such as electromechanical actuation, pneumatic, hydraulic, combination, or etc., and interactive, which reacts to the patient's signals and provides support by generating assistive force according to optimal assistance strategies.

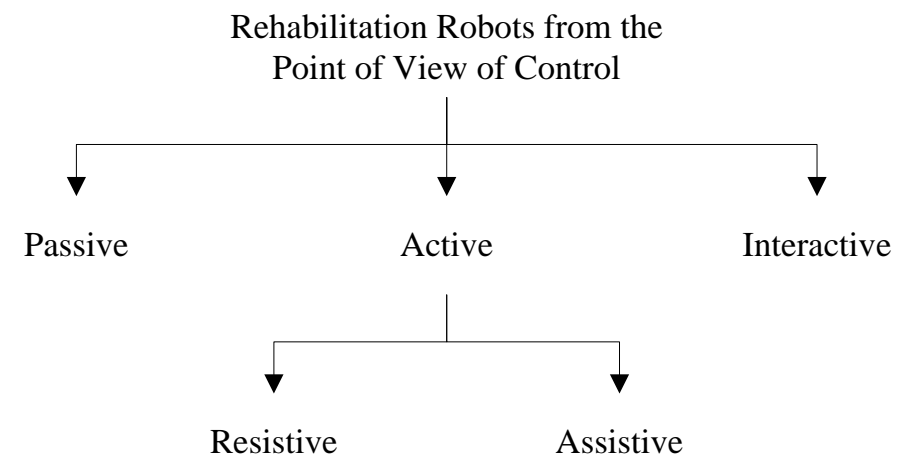

Figure 2. 4. Rehabilitation Robots Classification from the Point of View of Control and Therapeutic Approaches

In passive systems, limbs are passively stabilized or restricted in the predefined range of motion, which means that no actuation is employed to move the patients' limbs or to provide any assistance. Passive systems often consist of mechanical linkages such as pulleys, ropes, bearings, counter weights and different type of springs, which are easy to push. In contrary, active systems are equipped with actuators that provide support electromechanically, pneumatically or hydraulically and help patients to move the limbs actively. To provide assistance, they either employ open-loop control strategies or implement position-control systematic plans. Traditional position control schemes employ certain velocity profiles to take a limb from a predefined position 
to a new position. Interactive systems; however, not only benefit from actuators in order to provide active support but also employ sophisticated closed-loop strategies to react continuously to the patient's signals and efforts such that the assistive force is neither excessive nor insufficient. Interactive systems are often backdrivable; however, non-backdrivable systems can be useful too.

A backdrivable system would consume power during the entire time to move or hold the object, whereas a non-backdrivable system could maintain the desired position without power once it reaches the position [62]. Using backdrivable actuator for prosthetics has some difficulties. Since portable power sources have limited power capacity, they could not be used for a long time without recharge unless the prosthesis has access to permanent power sources. On the other hand, common characteristic of non-drivable systems such as having low, intrinsic, end point impedance makes the rehabilitation dynamic environment more interactive. They not only make the creation of dynamic rehabilitation tasks easier but also let therapists measure the subsequent effects following new interventions [33] [63] [64]. Using high-bandwidth force control for non-backdrivable robots [65] [66] [67] allows therapists to change the stiffness of joints easily with minimal friction and optimal power consumption, which in turn provides a free feel to the resultant motion [68].

\subsubsection{Therapeutic Approach and Physical Shapes}

Upper-limb rehabilitation robots from the point of view of therapeutic approaches and physical shapes can be grouped into two categories: end-effector-based and exoskeleton-based system, which is shown in Figure 2. 5. 


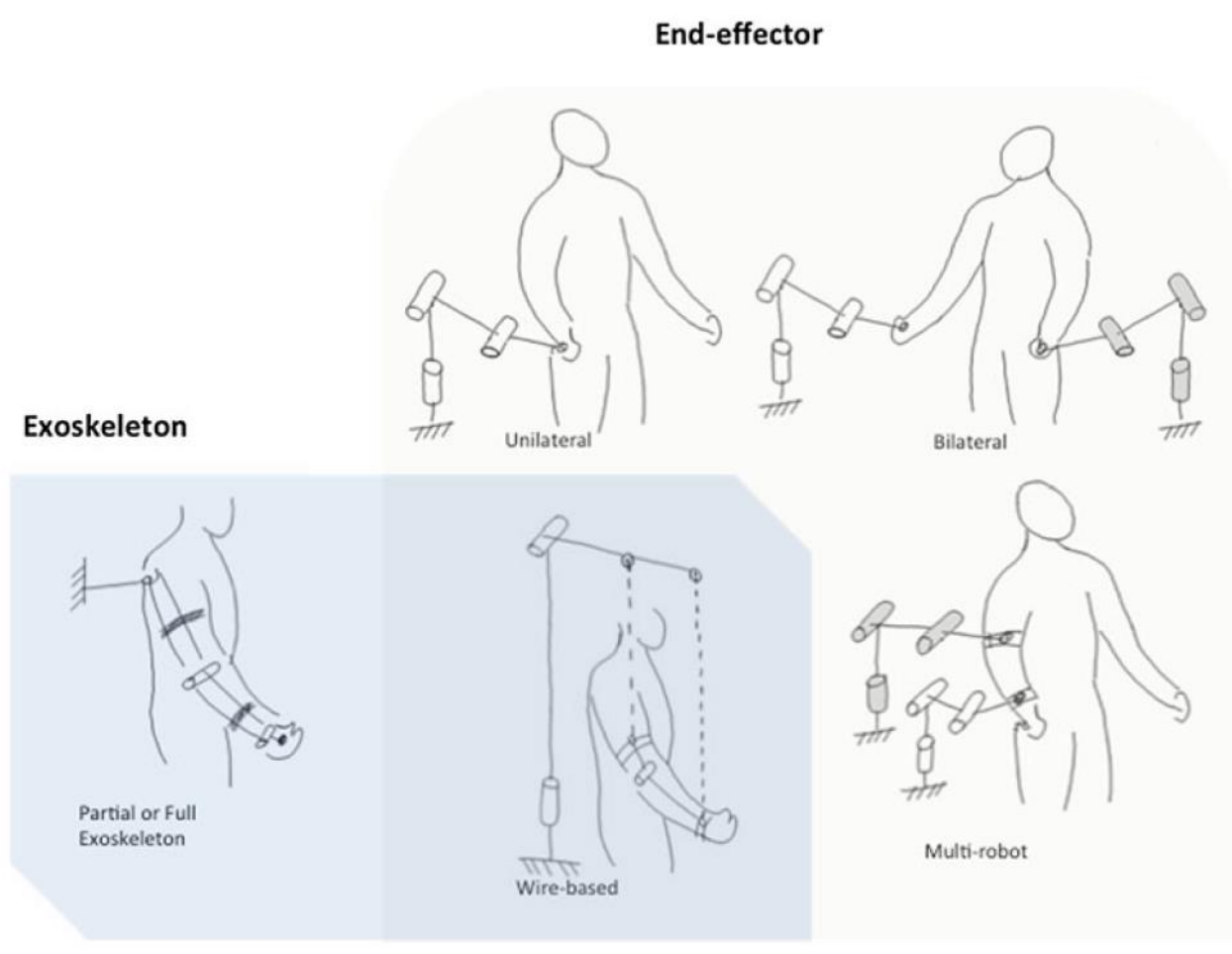

Figure 2. 5. Upper limb rehabilitation robot mechanical categories [69]

End-effector systems interact with the patient by means of orthosis attached to patients' forearm. End-effector systems are usually designed to deliver unilateral therapies. However, some end-effector systems deliver bilateral therapies by employing a second passive or active orthosis [69], which is attached to the non-paretic arm (e.g. MIME [70]and Bi-Manu-Track [71]). In exoskeleton systems, however, the arm is enclosed with a set of orthopedic mechanisms aligned with limbs, which requires the arm's joint to be fully-determined. This characteristic, when compared to end-effector systems, allows controlling the orientation of arm, where degrees of freedom exist. This means that there is a larger workspace for different tasks (e.g. Armin, L-Exos at Table 2. 1). However, joint misalignment and low performance are possible if the robot axes do not align perfectly with the patient's anatomical axes. On the other hand, end-effectors' joint about the wrist provide some limited degrees of freedom, which is not problematic for 2D planar tasks. It should be noted that wrist injuries might be the outcome of unsupported 3D spatial movements. 
Some recent exoskeleton and end-effector robot will be reviewed in this section following their pros and cons.

\subsubsection{End-Effector Robots}

MIT-MANUS robot, designed by Hogan and Krebs [64], was the first robot dedicated to evaluate the impact of using robot-aided therapy in upper-limb rehabilitation [72] [73].

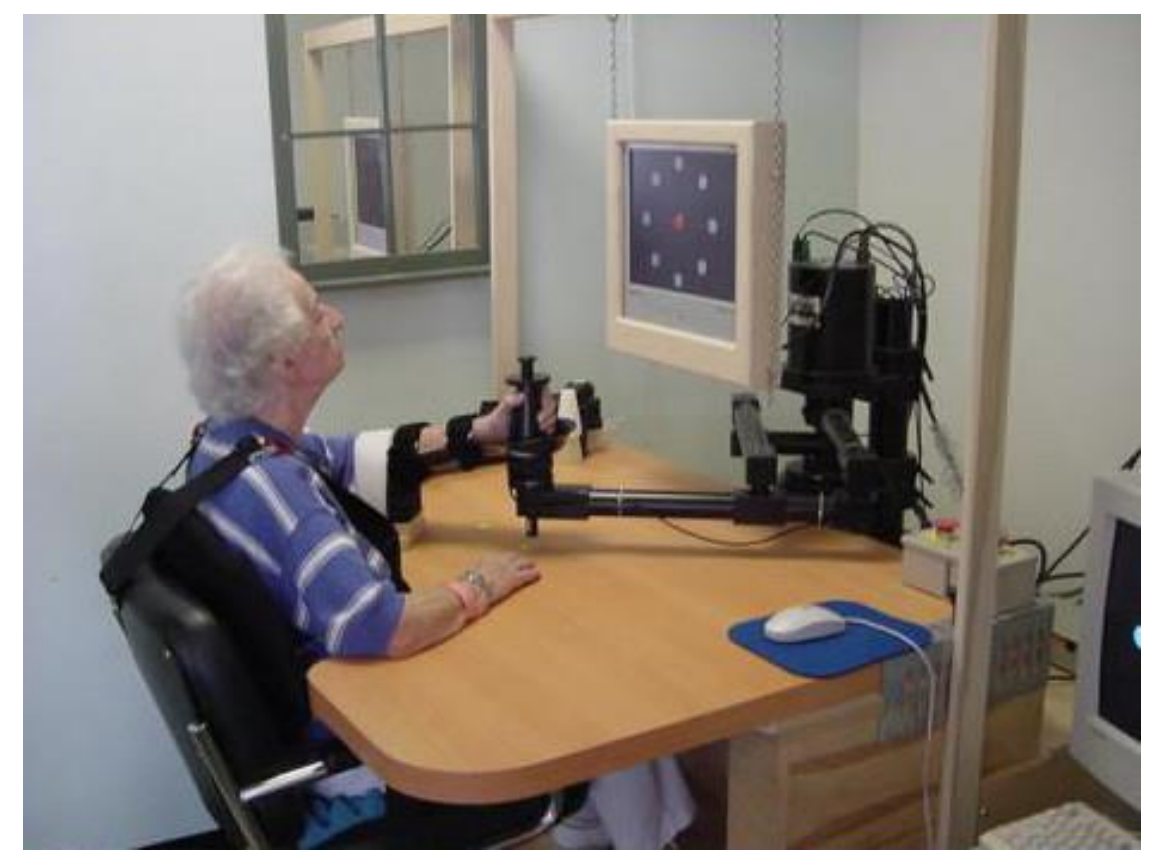

Figure 2. 6. Stroke-patient during therapy at the Burke Rehabilitation Hospital (White Plains, NY). Therapy is being conducted with a commercial version of MIT-MANUS (Interactive Motion Technologies, Inc., Cambridge, MA) [74].

MIT-MANUS robot (now commercially available as the InMotion2) is a planar SCARA-type manipulator, which provides horizontal plane movement therapy. It benefit from back-derivable actuators with low friction and inertia, which not only allows the robot to provide a smooth toptable movement but also lets the robot to use force feedback approach to transfer the force to the end-effector handle grasped by the patient. The required force varies according to the will of 
therapist. In order to increase the positive impact of the therapy, some applications such as 2D video games for tracing points or moving targets are integrated to the robot. Significant improvements are reported in both motor recovery gains following stoke [75] and chronic stroke recovery phase months after stroke [76] [77]. One of the bold outcomes of the previously mentioned research discredits the commonly accepted idea that the time for effective rehabilitation therapy is limited. The results show that brain retains the functional gains acquired during robotic therapy both after 3 month [77] and at the end of three years following initial treatment [72] [73]. The results not only suggest that the neuro-recovery process continued far beyond the commonly accepted 3-6 months post-stroke interval but also demonstrate that the lesion location plays a crucial role in neuro-recovery. As an end-effector robot, MIT-MANUS is limited for performing various therapies. The latest version (currently available as InMotion3 robot [78]) employs new extensions, which increases the capability to perform tasks for forearm and wrist assistance.

Innovative approaches have been employed to increase the efficacy of robot-aided therapy. For the first time, principles of symmetrical bilateral movement of the non-paretic and paretic arms were investigated at the VA Palo Alto Research and Stanford University, USA [79] [80]. The subsequent works proposed a 3D approach based on Mirror-Image Motion Enabler concept (MIME). MIME, as the first robot being able to serve unilateral/bilateral therapies [81], consists of a 6 DOF Puma 560 robot.

To perform the Mirror-Image Motion Enabler concept, the manipulator is linked to both limbs (paretic and non-paretic). From one side, it is coupled to a force and torque transducer on the splint of the paretic arm in order to provide support and assistance to the paretic arm, while the nonparetic arm is linked to a 6-DOF digitizer. In bilateral mode, once the patient moves the non-paretic arm, digitizer determines its position, which enables the robot to mirror the motion and moves the 
paretic arm subsequently. However, if the robot is set on unilateral mode, it can perform passive, active-assistive and active-resistive tasks.

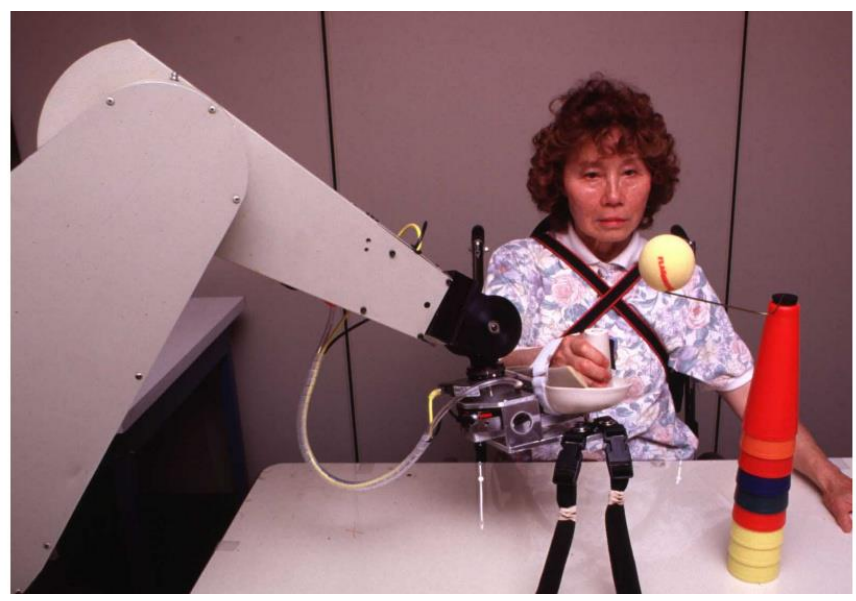

Figure 2. 7. MIME robot while delivering unilateral therapy [81]

Studies have shown that the Fugl-Meyer Assessment (FMA) gains are higher for the chronicstroke group, which experienced MIME therapy than the equivalent group experienced conventional neurodevelopment therapy [82].

The Bi-Manu-Track system [71] is another example of end-effector robots, which is able to be set on passive and active-resistive modes for both limbs and passive mode of the paretic limb to mirror the movement of the non-paretic limb [71].

The major movements provided by this robot are bilateral rehabilitation of the wrist flexion/extension and forearm pronation/supination. Such therapies, according to Hesse [71], are beneficial for reducing spasticity and improving motor control in chronic stroke patients. Similar works are based on the 'mirror image' hypothesis. It suggests that the damaged hemisphere of the brain might be influenced by the undamaged hemisphere while performing bilateral therapies [71] [83]. 


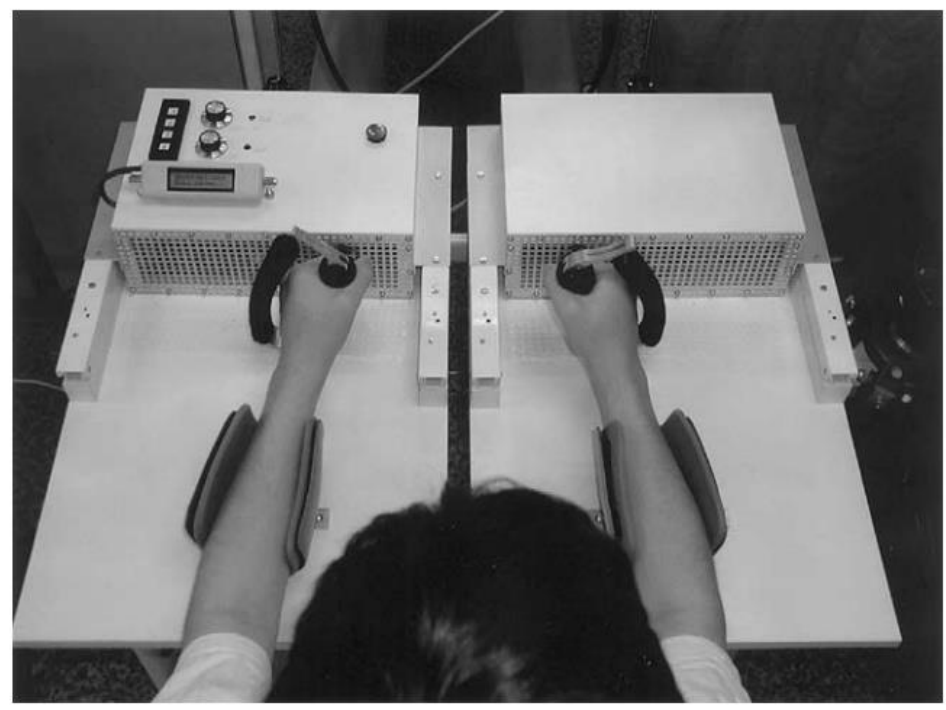

Figure 2. 8. Computer-assisted arm trainer; patient with left hemiparesis practices a repetitive bilateral pronation and supination movement of the forearm [71]

End-effector systems have achieved great accomplishments in stroke rehabilitation therapies during the past 20 years; however, their small range of motion has been always a limiting factor for ADL therapies. Using exoskeleton robots is the best alternative. They provide a larger work space to perform ADL tasks such as interacting with the environment while receiving position or force information in a more natural way.

\subsubsection{Exoskeleton Robots}

ARMin [84] is one of the first active robots designed specifically for arm therapy applications and ADL training. Arm rehabilitation therapy includes shoulder abduction/adduction, elbow flexion/extension, and forearm supination/pronation. ARMin has a semi-exoskeleton structure with six DOF, which is fixed to a fixed structure (wall) and because of bulkiness, it is only suitable for clinical applications. To perform therapeutic tasks, patients should sit underneath the device while the paretic arm is encapsulated on a distal exoskeleton orthosis. ARMin benefits from 
position, force and torque sensing/actuation, which are developed based on the cooperative concept. They facilitate patient cooperation when the patient power to perform the task is not adequate. To increase efficacy of therapy, audio and visual applications are integrated to the system by providing game-like exercises.
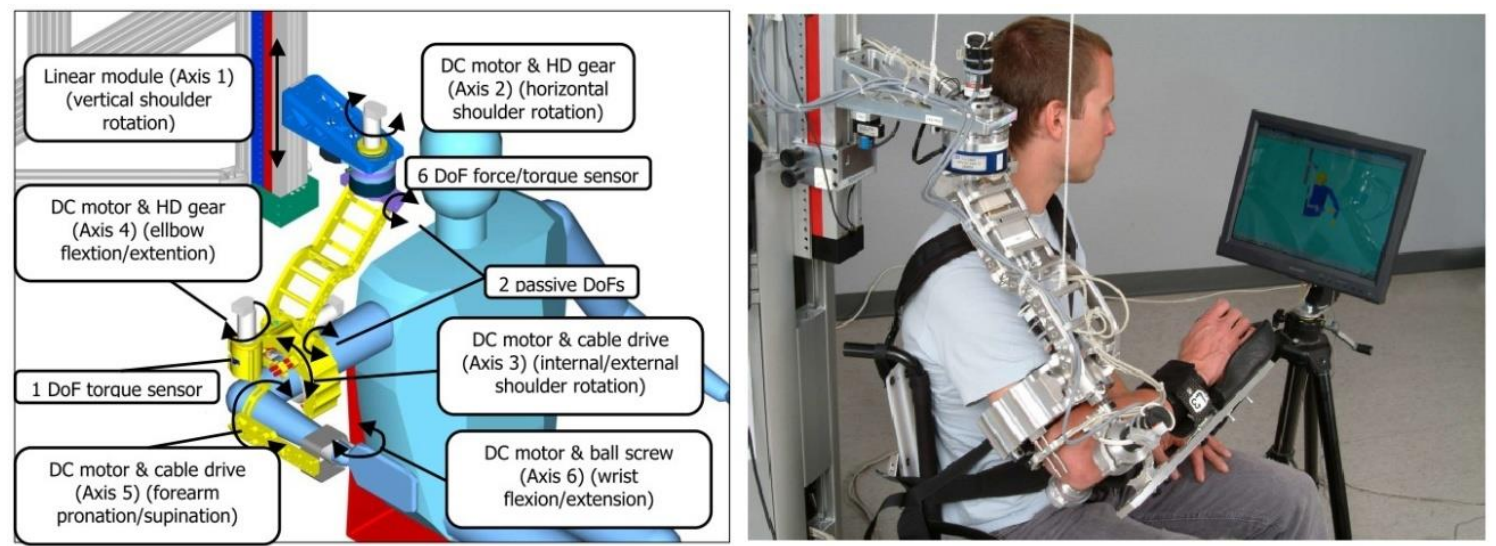

Figure 2. 9. (Left) ARMin Mechanical structure, sensors and motors, (right) ARMin with four active DOFs (shoulder and elbow) [85]

Clinical studies [86] have shown that the FMA gains achieved by using ARMin system is similar to end-effector systems, which shows the same level of efficacy for both systems.

Passive approach is also used for exoskeleton robots. Reinkensmeyer proposed a passive therapy based on the Java Therapy concept [54]. T-WREX [87] is an example of passive robots, which is now commercially available as ARMEO by HOCOMA, Switzerland. It employs a passive counter balance mechanism to compensate the gravity and the masses of the upper limbs. The major purpose of T-WREX is to provide passive assistance for low-strength muscle therapy. To adjust the system for different therapies and patients, additional elastic bands were used while grip sensors provided movement and grip force feedback. Similar to many rehabilitation robots, different computer games (eating, cracking eggs, etc.) with audio feedback were integrated to the system. Physiotherapists could also use "task progress charts" to track improvements of the loss 
functions [87]. Clinical studies comparing the efficacy of conventional therapy with T-WREX showed more improvements and acquiring higher FMA gains in chronic-stroke cases [88] [89].

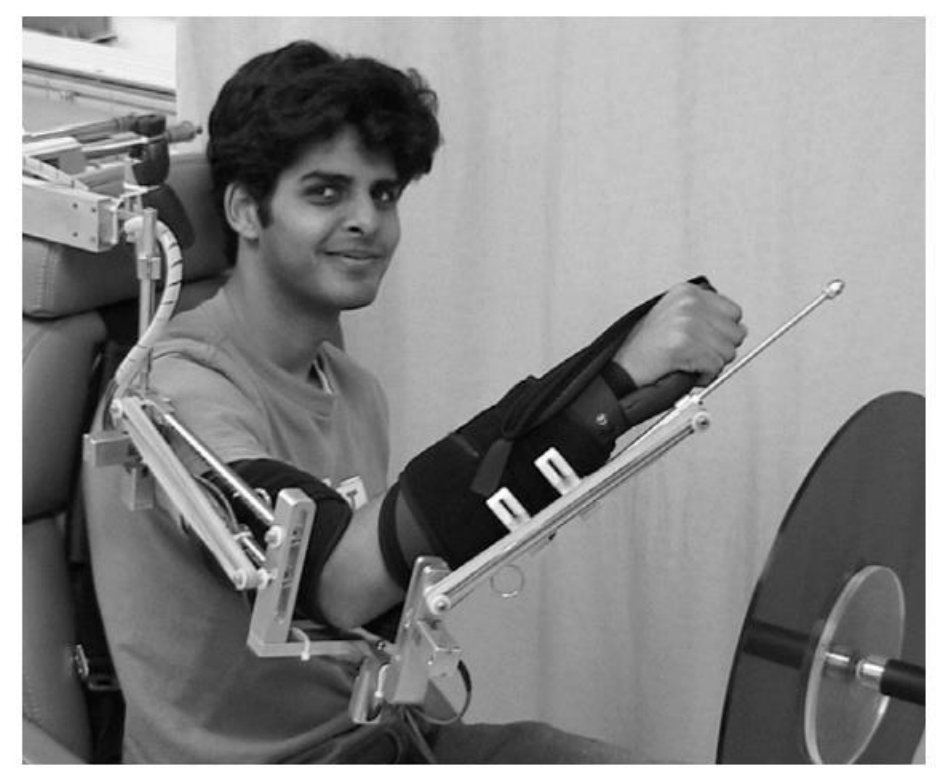

Figure 2. 10. Training-WREX [90]

A new passive approach using wires next to bed has been proposed for acute rehabilitation. Acute rehabilitation is an intensive program using multidisciplinary team approach to help the patient regain abilities after a major injury and return to everyday living. This approach is usually practiced in hospitals and under supervision of doctors and covers everything from safely eating to walking and talking properly and etc. Sub-acute level care; however, is less intensive than acute rehabilitation. The same combination of physical, occupational and speech therapy may be provided in the sub-acute setting. However, the number of hours each patient receives is less. An example of this type is NEuroREhabilitation roBOT (NeReBot) whose major application is for acute rehabilitation purposes [90].

Unlike other passive systems, NeReBot is relatively compact and semi-portable nevertheless it is still mostly used in hospital wards and under supervision of a physiotherapist. The NeReBot 
system is a 3-DOF robot, which is mounted on a wheeled frame while the patient lies under the system or sits on a chair. It consists of a forearm orthosis, which is controlled by 3 nylon wires. Similar to most of rehabilitation systems, it benefits from visual and audio feedbacks.
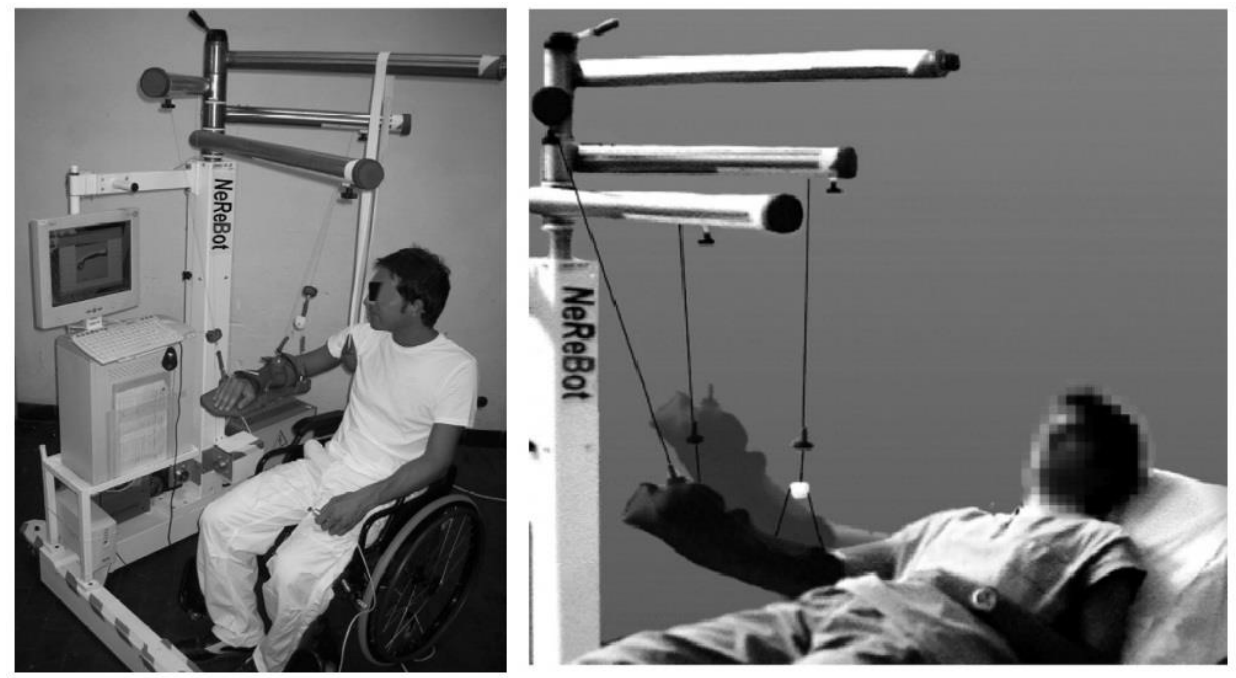

Figure 2. 11. The patient's right forearm is fastened into the splint to receive sensorimotor stimulation with NeReBot at the bedside position (left) in the sitting position (right). [90]

Clinical studies [90] over 35 acute stroke patients showed the same level of achievement of FMA gains as for MIT-MANUS.

While exoskeleton robots mimic the natural motions of humans, which is more practical and provides a greater range of motion, there is still a risk of injury during rehabilitation. Joint misalignment is the major cause of joint injury, which happens when the robot axes do not align correctly with patient's arm anatomical axes. To minimize the risk of injury of joint misalignment, a new method was proposed, which was based on designing an escorting system [91]. This new system, similar to all exoskeleton systems, supported ADL motions by supporting forearm and letting the upper limb move freely in special space. By adding two new passive joints, risk of injury of upper limbs caused by excessive forces on the shoulder was highly reduced. Existing exoskeleton robots have high rigidity, which not only limits the natural range of DOF of the 
patient's motion to the allowed directions but also interrupts the natural motion of upper limbs. To vary the compliance impedance of the new passive joints based on requirements, Redundant Drive Joints (RDJs) with adjustable stiffness and damping mechanism [92] [93] were integrated to the system and a high-frequency impedance control scheme [94] was proposed subsequently.

\subsubsection{Actuation and Power Transmission Approach}

The major and classic trade-off in design of portable exoskeleton robots is between power and weight [95]. Numerous actuation methods are proposed so far such as pneumatic actuators [96] [97], hydraulic actuators [98], ultrasonic motors [99], shape memory alloy wires [100] [101] and electroactive polymer actuators [102]. Despite advancement in robotic technology, designing light-weight and high-speed robots, which are able to mimic human motion is a challenge. They may satisfy one or some specifications such as actuation speed, grasping force, or low weight but not all of them. In addition, in many cases intermediate mechanisms such as reduction gears would increase weight inevitably.

Pneumatic, as one of the widely used type of actuators for rehabilitation and physical therapy [103], provides high power-to-weight ratio. Recently a trend toward using pneumatic actuators for physical therapy application emerged [104] [105] [106] [107] [108] [109] [110].

Pneumatic Muscle Actuator [111], known as the McKibben Pneumatic Artificial Muscle (PAM) [112] [113], is an actuator like a tube, which shrinks longitudinally when pressurized. The best example of this family of actuators, which is light and accurate is the McKibben-Muscle. They were invented by the physician, Joseph L. McKibben for polio patients in 1950s [112]. PAMs convert pneumatic force to pulling force and can provide fast and powerful response once fully pressurized. They have advantages over conventional pneumatic actuators such as high power to 
weight ratio, flexibility, and no mechanical parts. The other advantage of PAMs is their controllability since they only need one analog input while other conventional need two, one for each chamber.
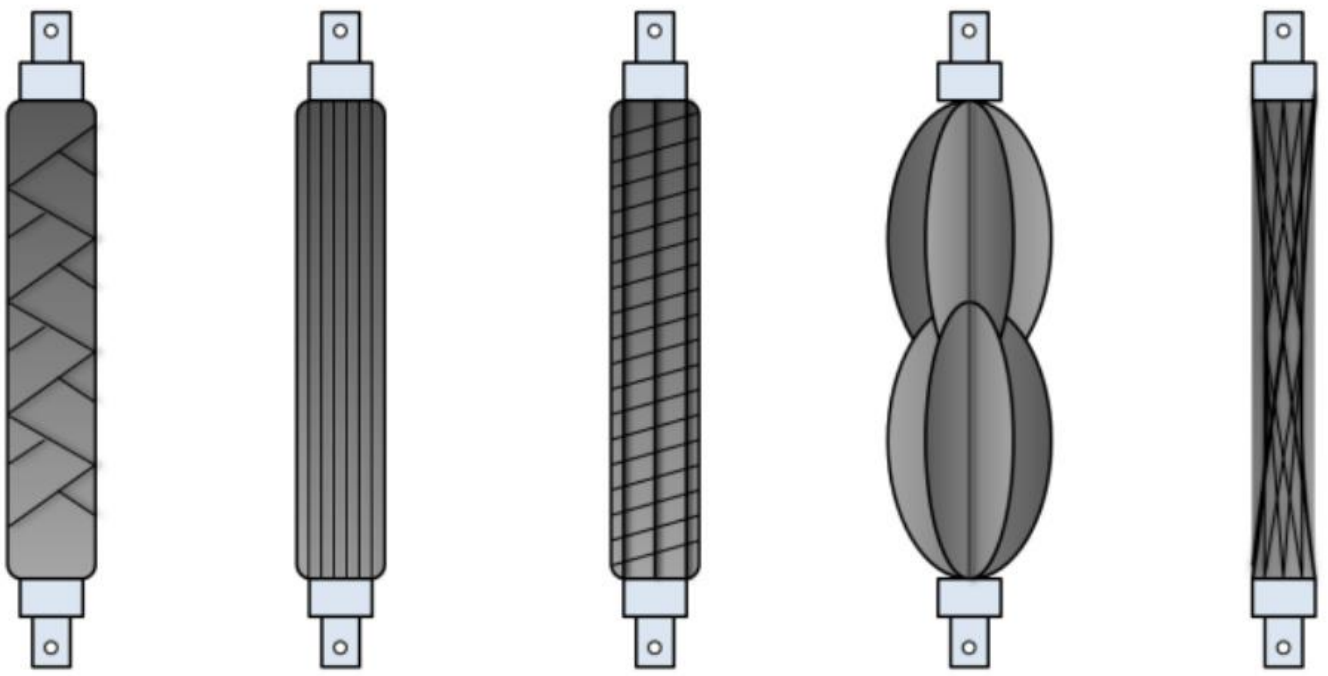

Figure 3. 1. Different shapes of McKibben Muscles [103]

Using this method, a muscle suit was made by Kobayashi group [114]. The robot did not have any metal frame and utilized compress air to actuate PAM. RUPERT [107] [108], a 5-DOF therapeutic robot, is another example, which was powered by 4 PAMs. RUPERT was used to provide therapeutic treatment for patients suffering from upper extremity disabilities. Despite high power-to-weight ratio of PAMs, their response are very poor in comparison with electric motors and they are heavy. However, pneumatic actuators are still being widely used for power amplification and providing static force instead of a dynamic force to perform tasks [114].

Most of exoskeletons, which need to perform in high bandwidths use motor-driven actuators. Unfortunately motors have low power-to-weight ratios, which limits the output force to perform certain tasks. 


\section{Cable Transmission Mechanism}

Using cables/tendons for force transmission, in particular for joints places far away from motors, has been used widely in the past two decades. Using cables for torque transmission not only reduces the friction and weight but also reduces motor backlash to zero and ensures a better backdrivability grade for the system [115]. In contrary, additional transformations are required to control open-ended cable-drive systems [116]. One major reason is that the number of joints required to control is less than the number of actuators. Since cable systems can only apply force through tension, it is necessary to have an antagonistic set of cables to achieve bidirectional movements. Thus, a minimum of $n+1$ cables are necessary for controlling $n$ joints. In addition, it is necessary to have a positive tension in all cables at all times to prevent the cables from becoming slack. Moreover, the load applied by cable motion to a series of pulleys, would affect multiple joints, which results in undesirable error.

Using cable for torque transmission has been also commercially used for rehabilitation robots. Johnson, Carus and et al. [117] developed MULOS (motorized upper-limb orthotic system), a fiveDOF electrically powered upper-limb orthosis, with three degrees of freedom at the shoulder, one at the elbow, and one to provide pronation/supination. The power transmission for the shoulder elevation was provided by tensioned cables. No cables were used for the elbow and forearm mechanisms because the required torque for the elbow joint was considerably lower than the value required for the shoulder joint. Thus, it was more reasonable to use motor at the joint place. In addition, the need for adjustments to fit the user would increase the design complexity. Thus, the cable transmission in this design was just used for one degree of the shoulder joint and the rest of joints were driven directly by electric motors. 
Rosen and Perry [118] [119] developed CADEN-7, a 7 degree-of-freedom anthropomorphic assistive robot, whose powered is generated by DC/brushless motors and power is fully transmitted by cables and pulleys.
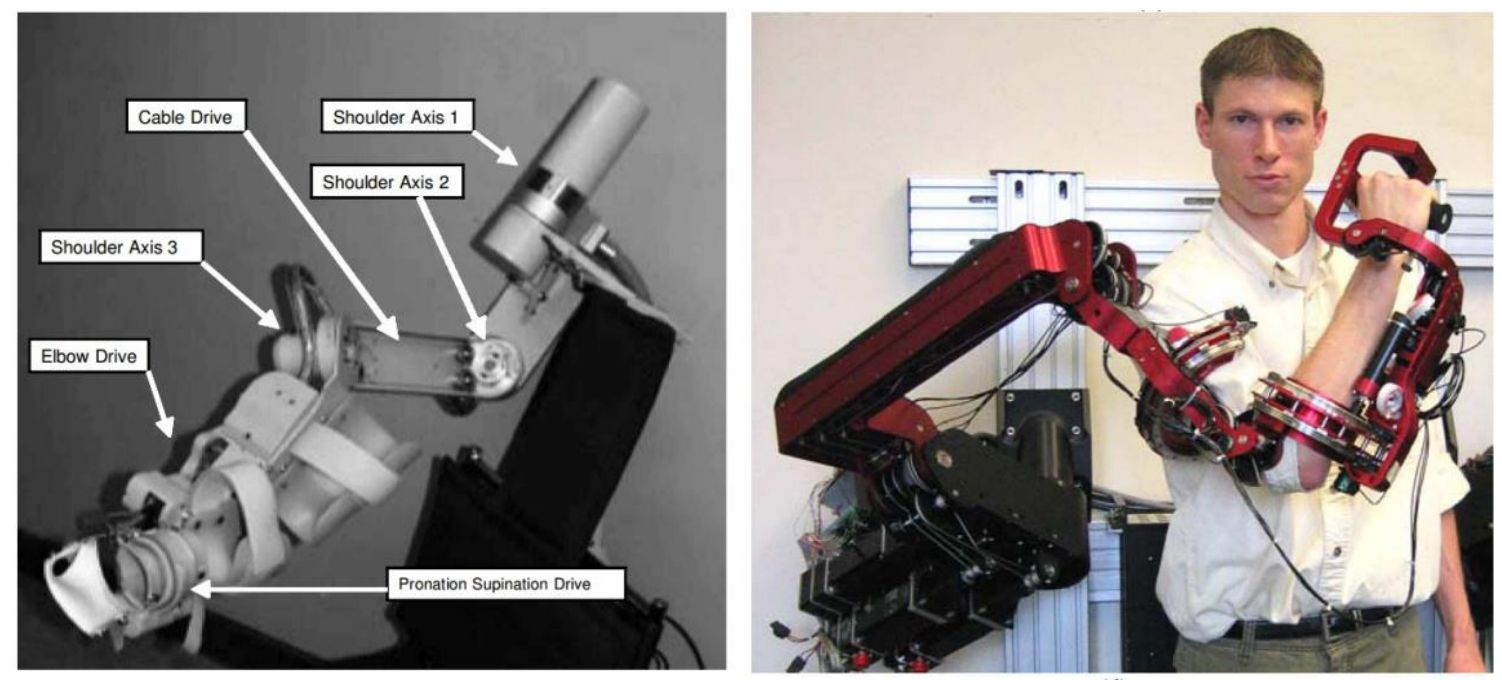

Figure 2. 12. (Left) Electric motors and cable drives arrangements at the shoulder mechanism [117] (Right) CADEN-7 [88]

Negligible backlash due to using cable for power transmission was the distinctive characteristic of this robot. However, the major challenge in cable driven devices was achieving mechanical joint, whose range of motions matches to those of the human arm.

MEDARM, a rehabilitation robot with 5 DOF at shoulder complex, was developed by Ball and et al. [120] whose power transmission was fully cable/belt based. The shoulder/elbow mechanism was a 4-DOF mechanism consisting of a 3-DOF glenohumeral joint and a single joint at the elbow. The power was provided by 5 electric motors on the base of the robot and transmitted by cable to the point of actuation. An extra motor used in this robot to keep a positive tension all over the robot. An additional break mechanism was used in this robot to always ensure positive tension along cables. 

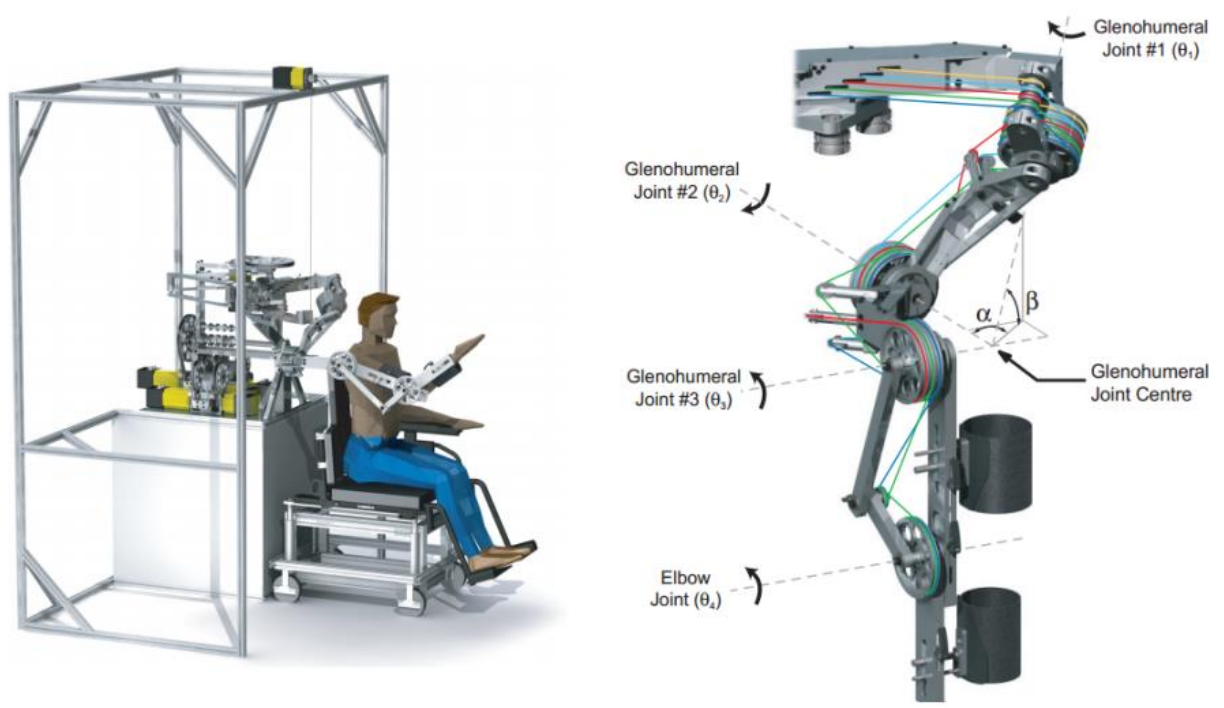

Figure 2. 13. (Left) MEDARM system consists of a 6DOF robotic exoskeleton mounted onto a support structure. (Right) A CAD drawing of the MEDARM mechanism showing the orientation of joint, cables and symbolic limbs masses.

ARMin [121] [85] [122] [84] is a whole-arm rehabilitation robot, which was designed by Riener and et al. in ETH, Switzerland. It is an exoskeleton/end-effector robot, which has 6 DOF whose forearm pronation and supination was actuated by cable transmission.

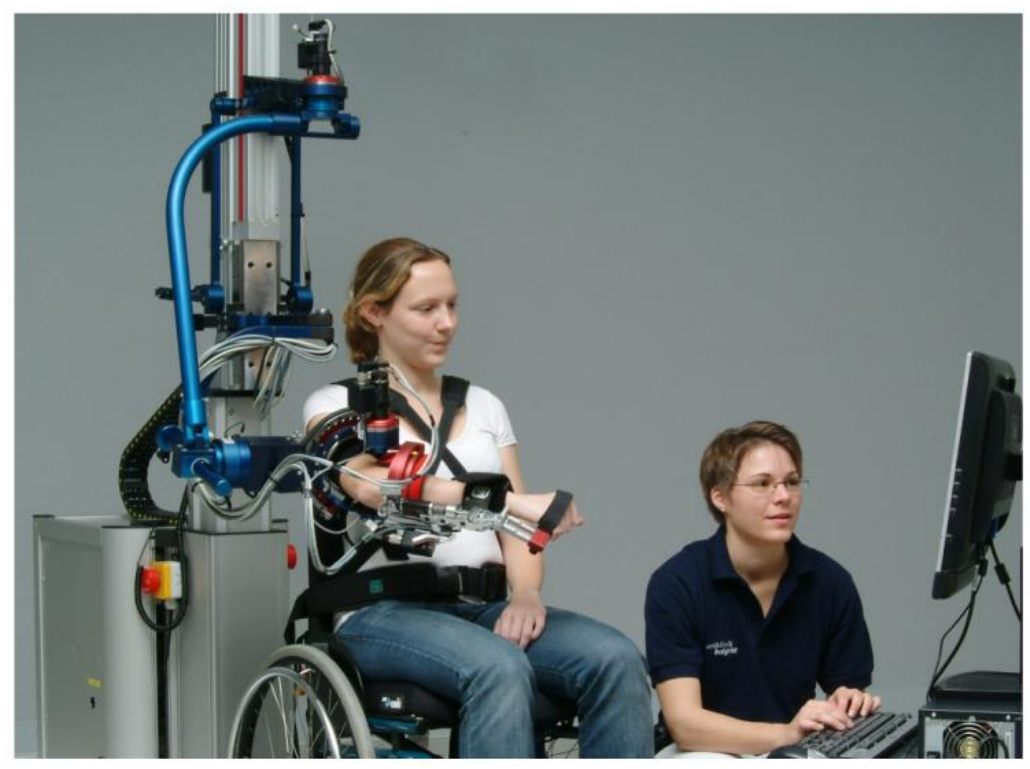

Figure 2. 14. ARMin II robot and a patient sitting on it. A physiotherapist is sitting nearby to select the rehabilitation procedure [86] 
Beside elbow and shoulder, cable transmission was used for hand and finger rehabilitation too. DiCicco, Matsuoka, and et al. [123] designed an EMG-controlled hand exoskeleton for natural pinching. To have full flexion/extension, all joints of the finger should move. To transmit force for flexion and extension separately, DiCicco used cables. These cables were pulled by a couple of pneumatic pistons acting in compression.

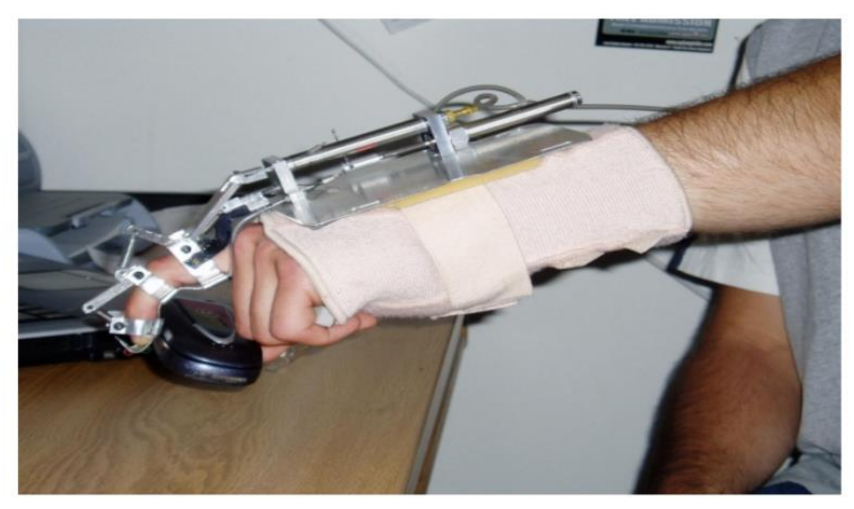

Figure 2. 15. Lightweight exoskeleton that allows basic pinching motion [123]

Various types of cable are used for force transmission in rehabilitation robots. Wege and Hommel [124] designed a hand exoskeleton and implemented a PID controller for the position control.

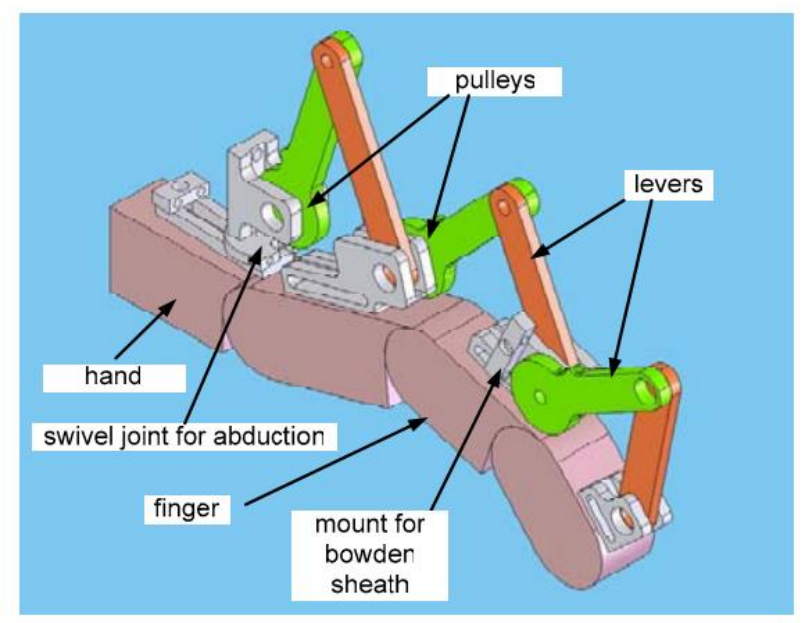

Figure 2. 16. Wege's finger exoskeleton CAD drawing. Two Bowden cables are used for each pulley to allow flexion and extension movement [124] 
Bowden cables were used to connect levers together and transmit force to the required place. The bidirectional movements of the robot were achieved by actuating motors, which were controlled by the control unit. The robot had 4 DOF and it could be easily attached on a finger and also be adjusted or deformed to the desired shape. DC motors with transmission gears pulled Bowden cables trough a pulley. Two Bowden sheaths for each joint were attached to a tension device to keep cables under tension reducing slackness.

Actuation solutions adopted in robotic hands have benefits and shortcomings. However, heaviness and slowness of such systems are still a major drawback. Bulkiness and heaviness of exoskeleton robot not only increase the production costs and power consumption but also make them perform slowly and inefficiently.

To resolve this issue, Würtz and etc [125] [126] proposed a novel tendon-based actuation method called "twisted string actuation system", which has been developed within DEXMART [127] [128] project to fit smaller actuators in robots. This method provides low torque, high speed actuation, which reduces the size of actuator substantially and allows designer to locate small actuator in the robot, while it inherits high grasping characteristics of cable-actuators. To ensure performance, Würtz and colleagues designed a finger prototype and controlled the robot by sliding manifold control law, which is equivalent to a high gain PID and guarantees the system robustness even in simplified models.

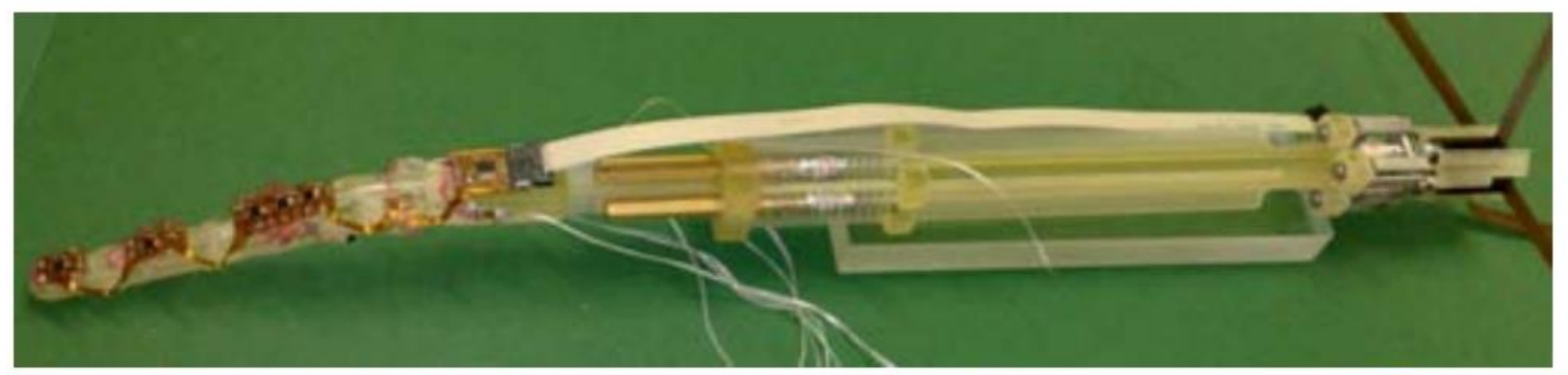

Figure 2. 17. Finger prototype actuated by the twisted string actuation system. [125] [126] 
A similar method called “Twist Drive Actuator” proposed by Sonoda and etc [129] [130] [131]. In the new method, the rotative motion of the motor was transmitted to the linear motion of long and thin tendons by means of very small high-speed motors without speed reducer or any intermediate mechanisms such as gearboxes, pulleys, or ball screws. The difference between the twisted string actuation and this method is only using very thin string with very high-speed actuators. Sonoda and colleagues modified their method [132] by including spring stiffness and providing a more dynamic model. They applied the new concept on a finger and used force control method for validation. The finger robot performed flexion and extension by an embedded spring at the outer side of the robot.

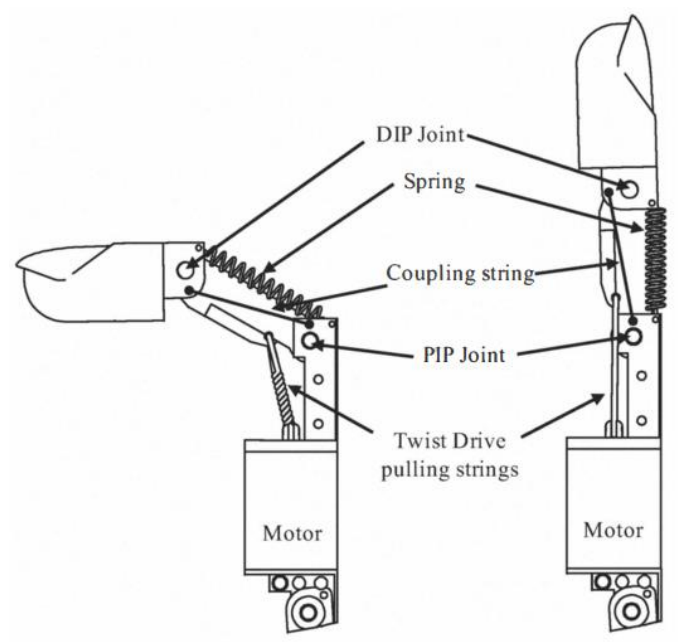

Figure 2. 18. Structure of the prototype of the finger robot designed by Sonada and his group [129] [130] [131]

Guzek and etc [133] used a similar twisting actuation mechanism but focused on providing a more accurate and reliable model by investigating the following effects on model: 1) the effects of strings properties such as diameter, Young's modulus, material, number of strings and, configuration of strings and their geometry 2) the effect of increasing the load on the response of the model, and 3) testing one set of strand for multiple times. 
Adopting twisting string actuation method, Aukes and his colleagues [134] designed a new class of multi-fingered robotic hands. Being able to grasp multiple objects and conform to their shapes as well as performing basic manipulation and benefiting from lockable degrees of freedom are the major characteristics of this manipulator. Similar to other cable driven finger robots, three independent degrees of freedom in the finger were driven by only one actuator. To balance the closing tendon force between each phalanx and provide return actuation, springs were attached across each joint and electrostatic braking at joints for locking.

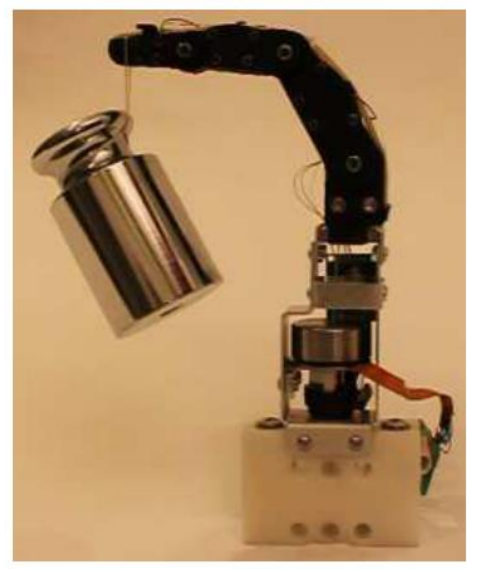

Figure 2. 19. Finger robot developed by Aukes while joints 2 and 3 are locked and holding $1 \mathrm{~kg}$ at the finger tip [134]

Similar to previous works, Shin, Lee, and colleagues [135] designed a finger robot combining the twisted string actuation method [125] [132] with a passive clutch mechanism and one small DC motor for actuation. Since the motion of fingers got fast, when no reaction force was applied on fingers (no grasping), a passive clutch was used to reduce the speed. Dual-mode twisting mechanism let the operator to move finger fast in Mode I or generate a large grasping force in Mode II. 

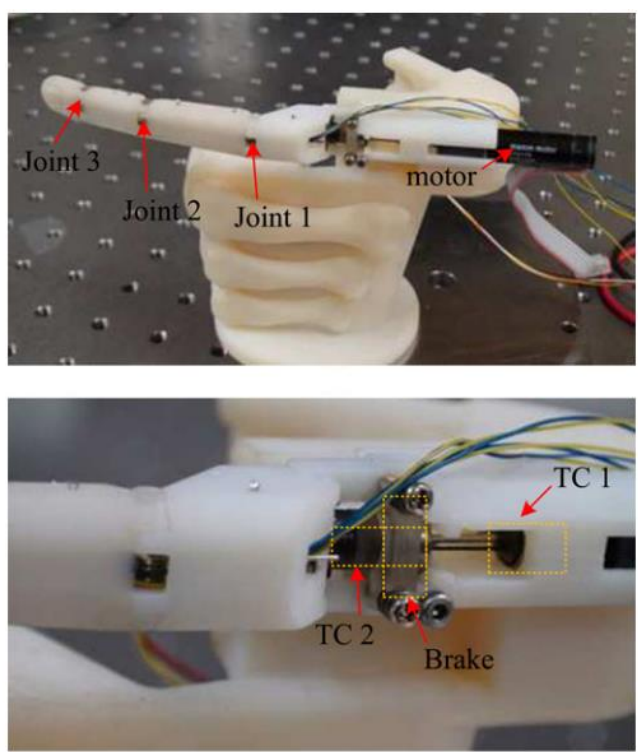

Figure 2. 20. Developed dual-mode robot finger by Shin [135] 
Table 2. 1. List of exoskeleton robots [69] [24] [136]

System and developer

\begin{tabular}{|c|c|c|c|}
\hline “ARMin”, ETH [84] & $\begin{array}{l}\text { Fixed exoskeleton, } \\
\text { unilateral }\end{array}$ & $\begin{array}{l}\text { Shoulder and elbow; } 4 \text { active, } 2 \text { passive } \\
\text { DOF }\end{array}$ & $\begin{array}{l}\text { Gravity compensated, massed practice with target, visual and audio feedback, } \\
\text { passive, active assistive. }\end{array}$ \\
\hline $\begin{array}{l}\text { "Dampace", University of } \\
\text { Twente, [137] }\end{array}$ & $\begin{array}{l}\text { Fixed exoskeleton, } \\
\text { unilateral }\end{array}$ & $\begin{array}{l}\text { Shoulder and elbow, } 5 \text { passive DOF ( } 3 \\
\text { shoulder, } 2 \text { elbow) }\end{array}$ & $\begin{array}{l}\text { Gravity compensated, visual and audio feedback. passive and resistive conrol } \\
\text { modes. }\end{array}$ \\
\hline $\begin{array}{l}\text { "Hand Mentor", Kinectic } \\
\text { Muscles, Inc [138] }\end{array}$ & $\begin{array}{l}\text { Portable, partial } \\
\text { exoskeleton, unilateral }\end{array}$ & $\begin{array}{l}\text { Wrist and hand, } 2 \text { active (wrist and } \\
\text { fingers flex-extension) DOF }\end{array}$ & $\begin{array}{l}\text { EMG and force feedback. Passive and active assisted modes. McKibben } \\
\text { pneumatic actuators. }\end{array}$ \\
\hline $\begin{array}{l}\text { "HWARD", University of } \\
\text { California, Irvine [139] }\end{array}$ & $\begin{array}{l}\text { Fixed, partial } \\
\text { exoskeleton, unilateral }\end{array}$ & $\begin{array}{l}\text { Wrist and hand, } 3 \text { active (1 wrist, } 1 \\
\text { fingers, and } 1 \text { thumb) }\end{array}$ & $\begin{array}{l}\text { Active assistive motion combining wrist extension with hand grasping and wrist } \\
\text { flexion with hand release. Visual and audio feedback. }\end{array}$ \\
\hline $\begin{array}{l}\text { "KIST", Korea Institute of } \\
\text { Science and Technology } \\
{[140]}\end{array}$ & $\begin{array}{l}\text { Portable, exoskeleton, } \\
\text { bilateral }\end{array}$ & $\begin{array}{l}\text { Shoulder, elbow, and wrist; } 7 \text { active, } 6 \\
\text { passive DOF }\end{array}$ & Passive, active assisted modes. Pneumatic and electric brake actuators. \\
\hline $\begin{array}{l}\text { "L-EXOS" (PERCRO) } \\
\text { Scuola Superiore [141] }\end{array}$ & $\begin{array}{l}\text { Fixed exoskeleton, } \\
\text { unilateral }\end{array}$ & $\begin{array}{l}\text { Shoulder and elbow; } 4 \text { active, } 1 \text { passive } \\
\text { DOF }\end{array}$ & Gravity compensated, visual and audio feedback, passive and active assistive. \\
\hline $\begin{array}{l}\text { "MGA", Georgetwon and } \\
\text { Maryland Univ. [142] }\end{array}$ & $\begin{array}{l}\text { Fixed exoskeleton, } \\
\text { unilateral }\end{array}$ & $\begin{array}{l}\text { Shoulder and elbow; } 4 \text { active, } 1 \text { passive } \\
\text { DOF }\end{array}$ & Gravity compensated, visual and audio feedback, passive, active assistive modes. \\
\hline $\begin{array}{l}\text { "Myomo e100”, Myomo, Inc. } \\
\text { [143] }\end{array}$ & $\begin{array}{l}\text { Portable, partial } \\
\text { exoskeleton, unilateral }\end{array}$ & Elbow, 1 active DOF & Active assistive motion using EMG biofeedback. \\
\hline $\begin{array}{l}\text { "Pneu- WREX", University } \\
\text { of California, Irvine [144] }\end{array}$ & $\begin{array}{l}\text { Fixed, exoskeleton, } \\
\text { unilateral }\end{array}$ & $\begin{array}{l}\text { Shoulder and elbow; } 4 \text { active, } 1 \text { passive } \\
\text { DOF }\end{array}$ & $\begin{array}{l}\text { Gravity mitigated, passive, active assistive. Visual and audio feedback. Based on } \\
\text { the T-WREX system. }\end{array}$ \\
\hline
\end{tabular}




\begin{tabular}{|c|c|c|c|}
\hline $\begin{array}{l}\text { "RUPERT", Arizona State } \\
\text { University [145] }\end{array}$ & $\begin{array}{l}\text { Fixed exoskeleton, } \\
\text { unilateral }\end{array}$ & $\begin{array}{l}\text { Shoulder, elbow, and wrist, } 4 \text { active } \\
\text { DOF ( } 1 \text { shoulder, } 1 \text { elbow, } 1 \text { forearm, } 1 \\
\text { wrist) }\end{array}$ & $\begin{array}{l}\text { Passive, active assisted. Online display of arm moving in the space. McKibben } \\
\text { pneumatic actuators. }\end{array}$ \\
\hline $\begin{array}{l}\text { "Rutgers Master II", Rutgers, } \\
\text { State University [146] }\end{array}$ & $\begin{array}{l}\text { Partial exoskeleton, } \\
\text { unilateral }\end{array}$ & $\begin{array}{l}\text { Hand, } 4 \text { active DOF (1 thumb, } 3 \\
\text { fingers) }\end{array}$ & $\begin{array}{l}\text { Active assisted, resistive modes. Visual, haptic and audio feedback. Custom } \\
\text { pneumatic actuators. }\end{array}$ \\
\hline $\begin{array}{l}\text { T-WREX University of } \\
\text { California, Irvine [87] }\end{array}$ & $\begin{array}{l}\text { Fixed exoskeleton, } \\
\text { unilateral }\end{array}$ & $\begin{array}{l}\text { Shoulder, elbow; } 5 \text { passive DOF ( } 3 \\
\text { shoulder, } 2 \text { elbow) }\end{array}$ & $\begin{array}{l}\text { Gravity mitigated, passive functional task training using visual and audio } \\
\text { feedback. Based on the Wilmington Robotic Exoskeleton. }\end{array}$ \\
\hline $\begin{array}{l}\text { Laboratoire Ampere UMR } \\
\text { CNRS, Czech Technical } \\
\text { University Prague [147] }\end{array}$ & $\begin{array}{l}\text { Fixed on wheel chair, } \\
\text { unilateral }\end{array}$ & $\begin{array}{l}\text { 4DOF shoulder joint and 3DOF elbow } \\
\text { joint }\end{array}$ & $\begin{array}{l}\text { Brushless DC motors, gear drives, force controlling with position feedback, high } \\
\text { ratio of DOF/weight }\end{array}$ \\
\hline $\begin{array}{l}\text { National Taiwan University } \\
\text { [148] }\end{array}$ & $\begin{array}{l}\text { Fixed to a stand or wall, } \\
\text { unilateral }\end{array}$ & $\begin{array}{l}6 \mathrm{DOF} \text { at shoulder } 1 \mathrm{DOF} \text { at the elbow, } \\
\text { and } 2 \mathrm{DOF} \text { at the wrist joint }\end{array}$ & $\begin{array}{l}\text { Rehabilitation robot for upper limb, DC motors, gear drives, electromyography } \\
\text { and force sensor feedback. }\end{array}$ \\
\hline McMaster University [149] & Fixed to wheel chair & 1DOF supination and pronation & $\begin{array}{l}\text { Stepper motor actuator and gear system transmission. To assist in ADLs (Assist } \\
\text { Carpal Tunnel Syndrome patients) }\end{array}$ \\
\hline Beihang University [150] & Portable, wearable & Thumb and index finger, 4DOF & separate actuation module, cables transmission. \\
\hline University of California - & Fixed to the wall, & 3DOF shoulder joint; 1DOF elbow & To assist shoulder, elbow and wrist motions rehabilitation, motor actuators, gear \\
\hline Santa Cruz [151] & unilateral and bilateral & joint; 3DOF wrist joint & system transmission, controller guarantees the asymptotic stability \\
\hline $\begin{array}{l}\text { American University of } \\
\text { Beirut [152] }\end{array}$ & Portable, wearable & 1DOF, elbow & $\begin{array}{l}\text { To assist arm motions, shape memory alloys actuators, force controlling } \\
\text { feedback. }\end{array}$ \\
\hline
\end{tabular}


CEA-LIST, Interactive

Robotics Unit [153]

Fixed to a stand or wall

$7 \mathrm{DOF}$

Wearable, portable, for

Bilateral Teleoperation

Zhejiang University [154]

Saga University [155]
Fixed to wall or stand

To assist workers and reduce musculoskeletal disorders, motor actuator, ball-

screws and cables actuator and transmission, allows true joint torque control

without force sensor

3DOF for shoulder, 1DOF for elbow

Pneumatic actuators, crank-slider mechanism and links, force and position

and 3DOF for wrist

sensors feedback, soft control interface

6 DOF; shoulder, elbow and wrist

joints; and forearm motion

DC servo motors, cable and gear transmission, for power assist purpose 


\section{Chapter 3: Human Anatomy and}

\section{Mechanical Design}

\subsection{Introduction}

In this chapter human upper extremities anatomy and anthropometry were considered and based on the physical limitations and characteristics of human motion, a wearable mechanical robot was designed.

The first section of this chapter is dedicated to human upper extremities anatomy. This is of the importance that any exoskeleton robot should fulfil the natural motion of the limbs. Thus, considering physical limitations of human anatomy such as bones, joint components, degrees of freedom, range of motion, and etc. is very crucial. In the next section of this chapter human anthropometry was studied. Any wearable robot should be adjustable to a wide range of patients. 
Thus, the targeted population of patients considering the anthropomorphic data of the target population such as height, weight, age, and etc. was determined. Considering the upper extremities anatomy and anthropomorphic data a one-DOF prototype for the elbow joint was proposed fabricated. The assistive prototype device was light weight and was able to assist stroke patients to perform regular motion in the range of motion of the elbow joint. Based on the results acquired from the first prototype, the final design with four DOF for shoulder and elbow assist was proposed, which had two actuated degrees of freedom for elbow and shoulder and two passive degrees of freedom.

\subsection{Human Anatomy [24]}

Human upper extremities consist of three major joint complexes: shoulder complex, elbow complex, and wrist joint.
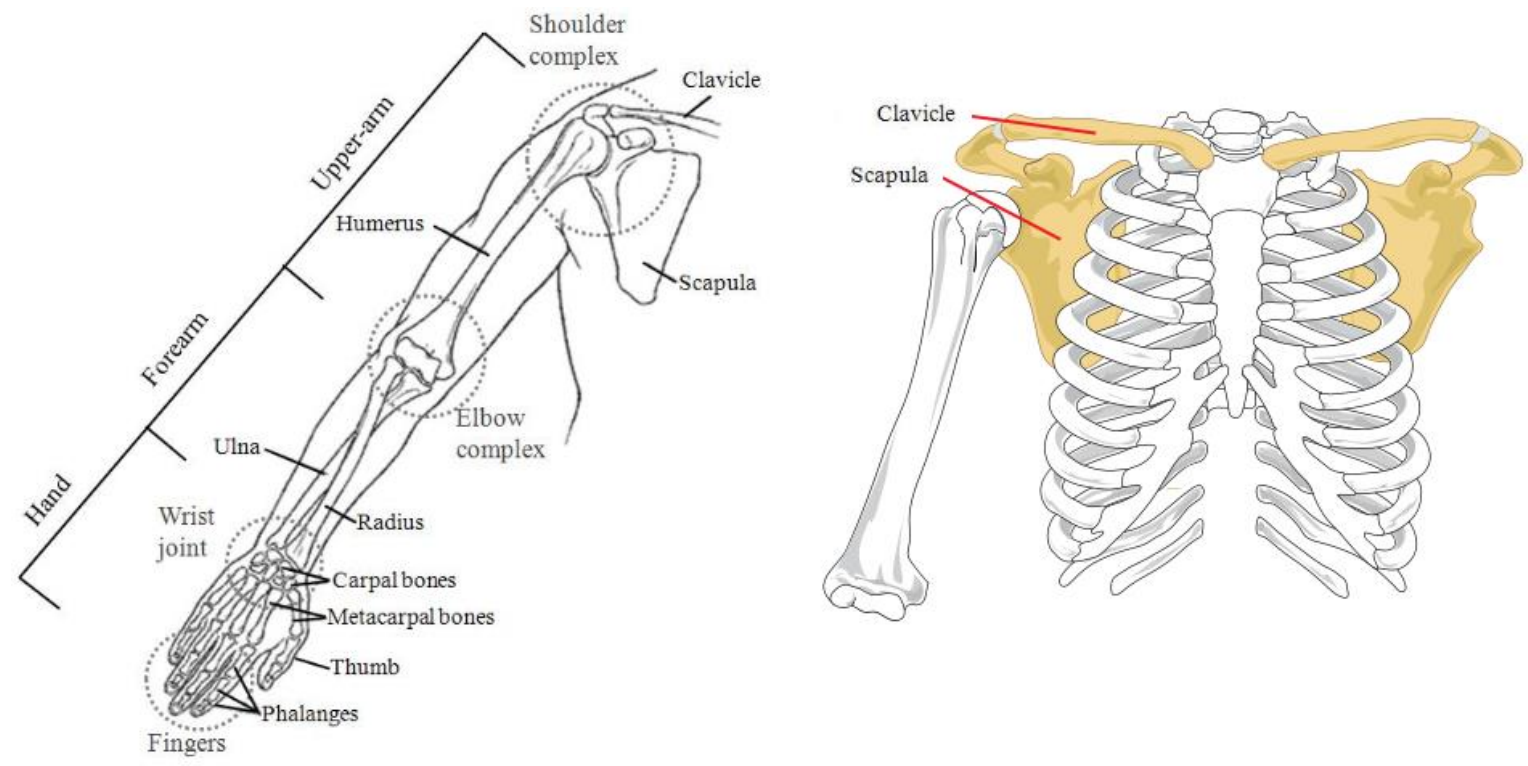

Figure 3. 2. The schematic demonstration of the upper extremities and thorax [24] [156] 
Human upper extremities have seven major degrees of freedom (DOF), three for the shoulder, two for the elbow, and two for the wrist. Since the proposed robot will be used for the purpose of shoulder and elbow assist, wrist joint anatomy will not be discussed. The sequence of rotations of the upper-extremity joints, which are commonly used in the biomechanics community [157], is shown below.

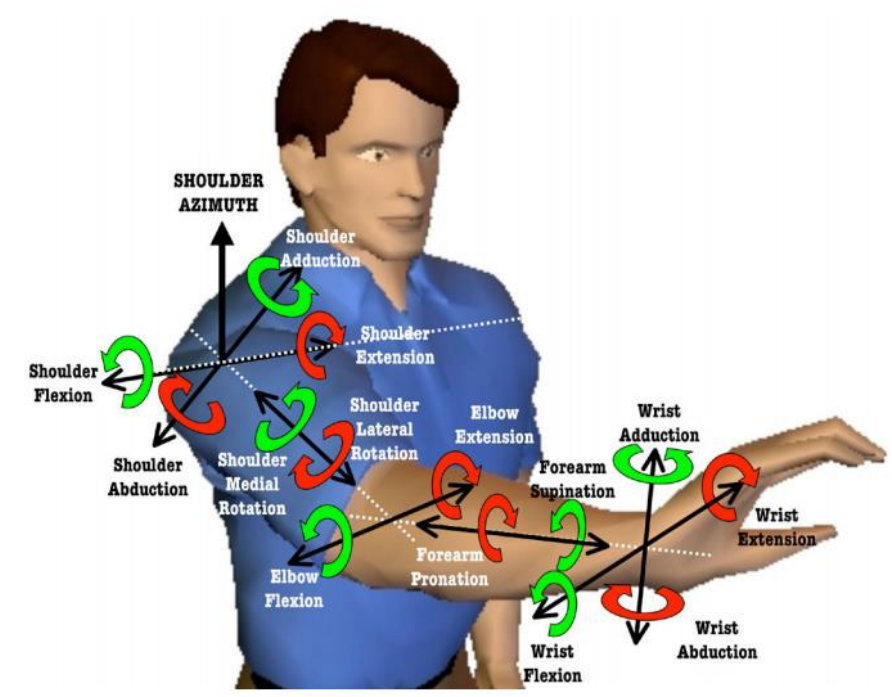

Figure 3. 3. Seven major degrees of freedom of the upper-extremities [158]

These seven major degrees of freedom can be categorized into motions in three planes as: Sagittal plane, Frontal plane, and Transverse plane, which will be eventually used to explain the range of motion. 


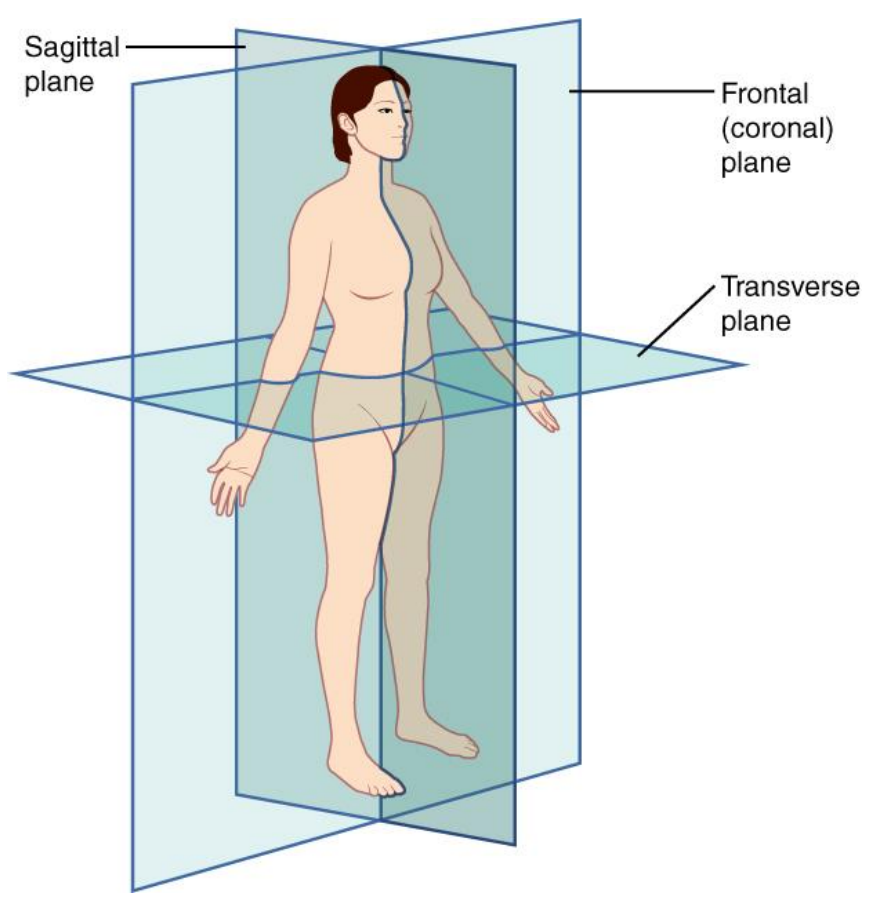

Figure 3. 4. Body planes [159]

\section{Shoulder Complex}

Shoulder complex is a compound joint consisting of three major bones: clavicle, scapula and humerus, which meet at the glenohumeral (GH) joint commonly referred as the shoulder joint. Shoulder joint is a highly mobile ball and socket joint [160] [161]. The sequence of joints are shown below.
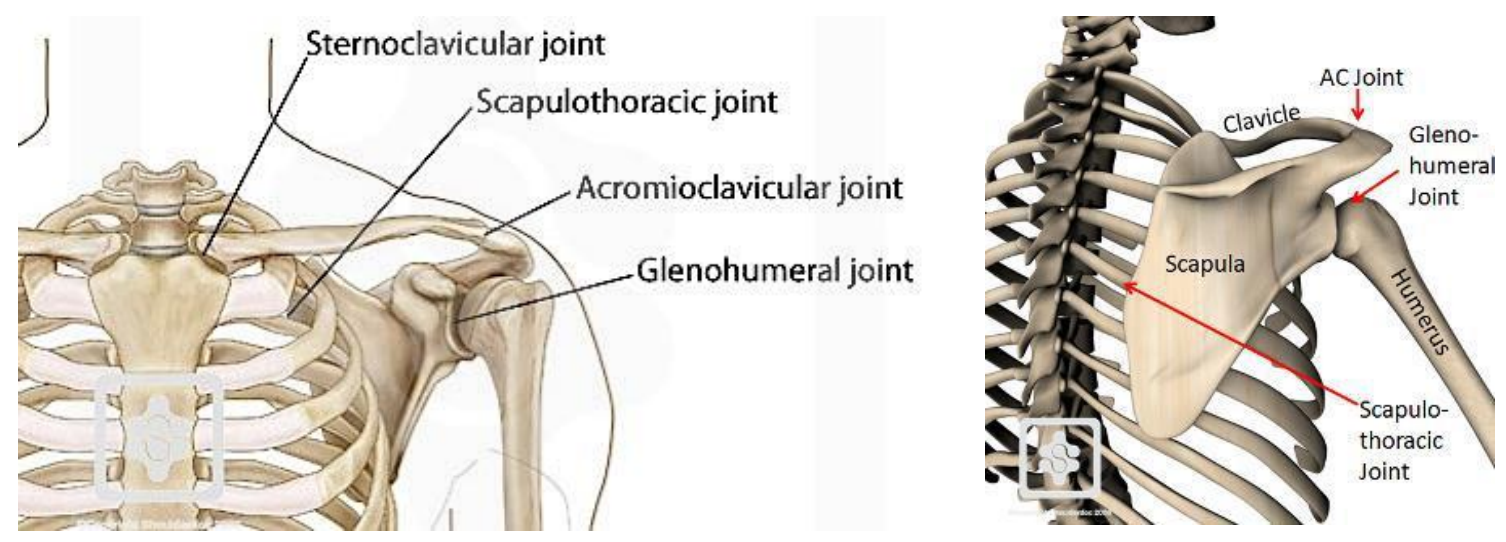

Figure 3. 5. The components of the shoulder joint [162] [163] 
The major motions of the shoulder complex are shoulder flexion/extension, shoulder abduction/adduction, and internal/external rotation.

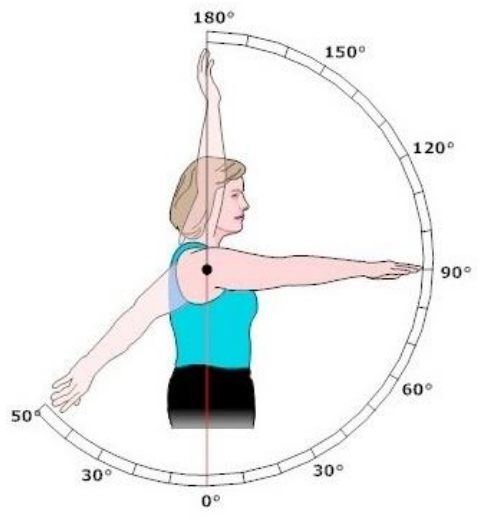

(a)

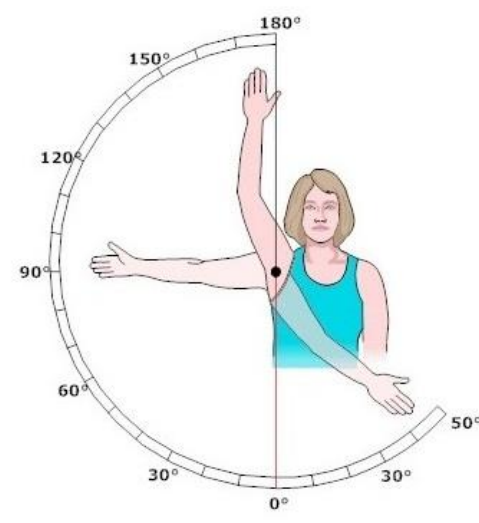

(b)

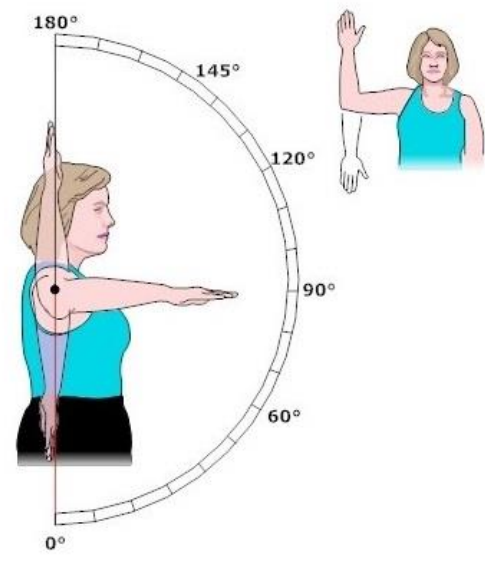

(c)

Figure 3. 6. References of shoulder motion, (a) shoulder flexion/extension, (b) shoulder abduction/adduction, and (c) shoulder rotation [164] [165]

The range of motion of the shoulder joints are: abduction $\left(180^{\circ}\right)$ and adduction $\left(45^{\circ}\right)$; extension $\left(45^{\circ}\right)$ and flexion $\left(90^{\circ}\right)$; and internal rotation $\left(55^{\circ}\right)$ and external rotation $\left(45^{\circ}\right)$ [166] [167]. Shoulder complex can provide a large range of movement and due to its high flexibility, designing any mechanical device for the shoulder joint needs special considerations. The motion pattern can be described through the motion of scapulothoracic joint (the joint between scapula and thorax) and glenohumeral joint (shoulder joint). This pattern is called scapulohumeral rhythm [168]. The scapulothoracic joint is a bone-muscle-bone articulation, which is not synovial (known as a diarthrosis, the most movable joint such as elbow in the body of mammals [169]). However, it is widely considered as a joint when describing the motion of the scapula over the thorax [24]. This means that the motion of the arm is not independent of the motion of the scapula. In other words, since there is a median joint between thorax and arm, shoulder joint is not attached to the body skeleton (thorax) through a so called ball-and-socket joint. According to the scapulohumeral 
rhythm, in the initial $60^{\circ}$ in the sagittal plane or $30^{\circ}$ in the frontal plane, scapula may remain stationary or may slightly move upward or downward. The major feeding activities such as using a fork or spoon or drinking are performed in this range of motion including shoulder elevation. These activities occur within the setting phase, which is $5^{\circ}$ to $30^{\circ}$ shoulder abduction and $5^{\circ}$ to $45^{\circ}$ shoulder flexion [168]. Following the setting phase, from the remaining angle to $170^{\circ}$, there is a predictable phase in which the scapula moves upward and it increases as the angle approaches $170^{\circ}[168]$ [170] [171]. As a rule of thumb for every two degrees of motion for the glenohumeral joint, scapular joint moves one degree [168]. This movement consists of a primary upward rotation of the scapula complemented with two consequent secondary motion, posterior tilting and rotation on the sagittal and transverse plane subsequently as the humeral angle increases. This is an important issue in the design of a wearable robot, since not many exoskeleton robots considered the motion of this joint in the design process and mostly ignore it or assume it is negligible. There are recent efforts to design shoulder mechanism, which cover full range of motion of the scapula [158]. If the desired range of the motion is in the stable range of motion of the scapula; however, increasing complexity of the robot for the sake of covering the whole motion is pointless. Since the targeted ADLs for our robot is in the stable range of motion of the scapula, scapular motion is neglected in our proposed design.

\section{Elbow Joint}

Elbow joint is a synovial hinge joint [172] between the latter end of humerus in the upper arm and the radius and ulna in the forearm, which allows hand to be moved towards and away from body [160] [173] [174] [175]. 


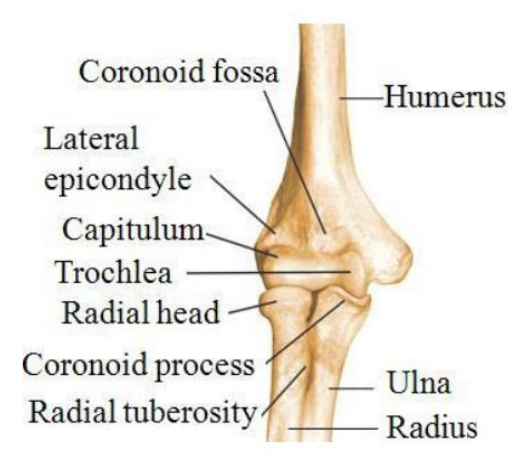

(a)

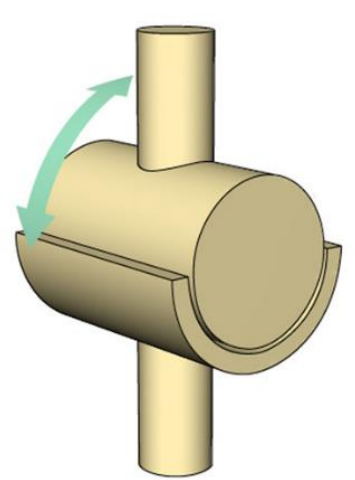

(b)

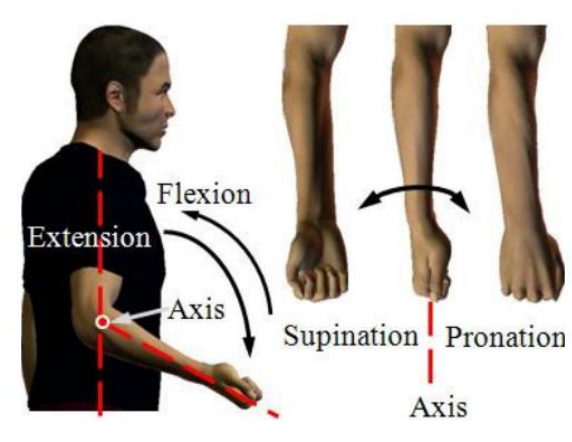

(c)

Figure 3. 7. (a) Elbow complex, (b) hinged joint, and (c) motion provided by the elbow complex [176] [24]

Elbow complex, similar to shoulder complex, is a compound joint consisting of two joints: the humeroradial and humeroulnar. The humeroradial joint is a limited ball-and-socket, hinge type of synovial joint located between the head of the radius and the capitulum of the humerus [177]. The elbow complex allows $2 \mathrm{DOF}$, flexion $\left(135^{\circ}\right) /$ extension $\left(0^{\circ}\right)$ and supination $\left(90^{\circ}\right) /$ pronation $\left(90^{\circ}\right)[166][167]$.

\subsection{Human Anthropometry [178]}

American Anthropological Association defines anthropology as "the science of studying present and past of humans, where the knowledge from the social and biological sciences meet the humanities and physical sciences" [179]. Anthropometry, however, as the major branch of anthropology, is the science, which defines "the physical measures of a person's size, form, and functional capacities" [180]. Such anthropometric measurements are used to evaluate the interaction between human and machines, tools, vehicles, and etc. The major impetus of such studies in the past was from historical purposes. In the past several years, however, the major purposes has shifted to the man-machine interfaces. 


\section{Segment Dimensions}

The easiest way to describe the size of human body is through the length of segments between joints, which varies with sex, age, race, and body type.

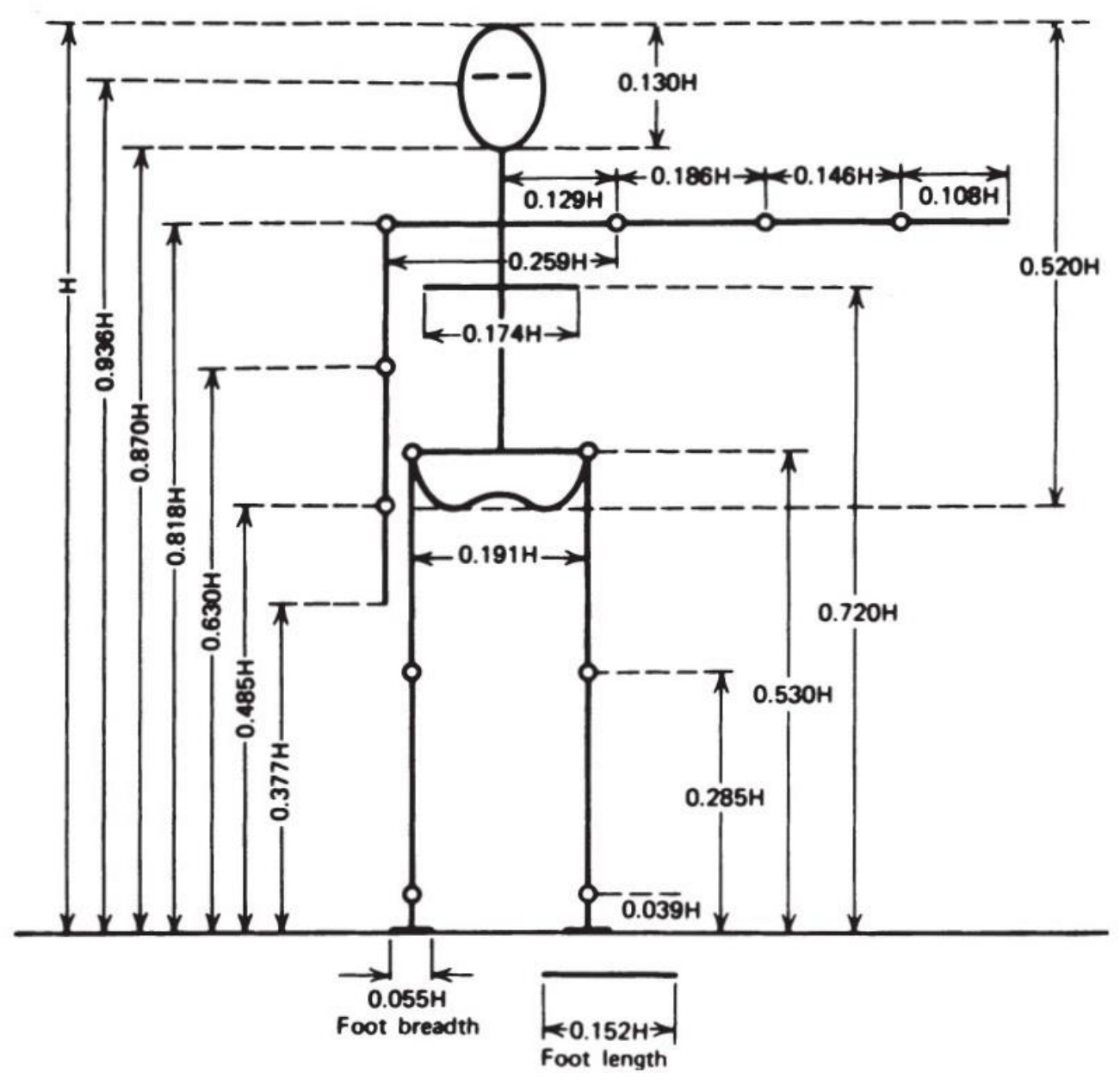

Figure 3. 8. Segments length as a function of height [178]

The first model with estimates of segment lengths and joint locations was proposed by Dempster et al. [181] [182]. Drillis and Contini prepared the first model of the average segment length as a function of height [183] [184]. The average segment proportions shown in Figure 3. 8 can be used to estimate the sizes of robot segments based on the range of users' segment sizes. It should be noted that these ratios are average values and do not replace actual data from individuals. 


\section{Segment Mass, Center of Mass}

Kinetic and kinematic analyses require data regarding center of mass and moment of inertia. Like the dimension of each segment, the mass of each individual segment increases as the total body mass increases. The location of the center of mass of each segment could be presented as a percentage of the length of the segment from the distal or proximal end of the segment. Mass and center of mass of each segment as a function of total body mass, and each segment total length are shown at Table 3.1

\section{Moment of Inertia}

Center of mass location of a system is needed in order to do the motion analysis. If acceleration is involved in the system, however, Newton's second law must be used for system analysis, which requires new properties of the system such as moment of inertia. Moment of inertia of limbs are calculated using radius of gyration. The average weight, center of gravity and length of targeted limbs of upper extremities are provided in Table 3. 1

Table 3. 1. Weight, center of gravity, length, and radius of gyration of targeted limbs of upper extremities [178]

\begin{tabular}{lcccc}
\multicolumn{1}{c}{ Segment } & Upper arm & Forearm & Hand & Forearm and hand \\
\hline $\begin{array}{l}\text { Definition of Segment } \\
\text { Glenohumeral } \\
\text { axis/elbow axis }\end{array}$ & $\begin{array}{c}\text { From Elbow axis to } \\
\text { ulnar styloid }\end{array}$ & $\begin{array}{c}\text { From Wrist axis to } \\
\text { knuckle II middle } \\
\text { finger }\end{array}$ & $\begin{array}{c}\text { From Elbow axis } \\
\text { to ulnar styloid }\end{array}$ \\
\hline $\begin{array}{l}\text { Weight Ratio: } \\
\text { Segment / Total Body }\end{array}$ & 0.028 & 0.016 & 0.006 & 0.022 \\
\hline $\begin{array}{l}\text { Center of Mass Ratio: } \\
\begin{array}{l}\text { Segment CG(Proximal) } \\
\text { / Segment Length }\end{array}\end{array}$ & 0.436 & 0.430 & 0.506 & 0.682 \\
\hline $\begin{array}{l}\text { Length Ratio: } \\
\text { Segment/Height }\end{array}$ & 0.186 & 0.146 & 0.108 & 0.254 \\
\hline $\begin{array}{l}\text { Radius of Gyration / } \\
\text { Segment Length } \\
\text { (About CG) }\end{array}$ & 0.322 & 0.303 & 0.297 & 0.468
\end{tabular}




\subsection{Exoskeleton Robot Design Criteria from the Anatomical View Point}

Upper-limb exoskeleton robots have different applications such as rehabilitation, motion assistance, human power amplification and others. According to the application, they should satisfy different requirements. However, all different types should satisfy some important general requirements.

Safety is a very important issue in designing any system interacting directly with human users. Special consideration must be taken such as mechanical breaks for the range of motion, mechanical and software emergency shut-offs, and alignment with human joints [24]. In addition, if the robot must be attached to a support, the mechanism should still be able to provide anthropomorphic motion. Exoskeleton robots often have deviation from the right angle of the human joint. In other words, if the exoskeleton joints are not aligned with human joints, they can cause serious damages to joints. Therefore, extra mechanism should be considered to cancel out the ill effect caused by the mechanism or the exoskeleton robot should be strapped to corresponding limbs such that they do not deviate significantly [185]. Furthermore, robot is located on the skin (not on the imaginary line between the centers of rotation of joint), so there will be a gap between the center of rotation of the robot segments and that of the human limb. This gap must be considered in designing the joints of the exoskeleton especially for complex joints with several degrees of freedom like shoulder [186].

Shoulder complex is one of the most anatomically complex parts of the human body. Thus, designing a ball-and-socket joint like the shoulder complex requires special considerations. Motion singularities due to physical offset and mathematical modeling of such joints are some of the major obstacles dealing with shoulder joint. An exoskeleton for the shoulder joint should not 
only fulfill the physical range of motion of the joint but also avoid interference with natural motion of the joint [158] [187] [188]. Moreover, center of rotation of the shoulder joint changes with motion. This motion is a function of the surfaces of the joint and scapular motion. The effect of the surface of joints on the shoulder motion can be neglected. However, as discussed before, the effect of scapular motion on the shoulder motion can be significant, if it exceeds a certain bound. Thus, an ADL with range of motion bigger than the targeted range should be performed very cautiously to avoid further physical injuries to shoulder, scapula, or thorax.

In the case of elbow joint, three bones (humerus, ulna, and radius) are involved. The elbow joint can be modeled as a uniaxial hinge joint [189]. Since the robot's axis of rotation is located at the user's elbow joint, it can be modeled as a simple revolute joint.

In order to provide proper assistance, rehabilitation robots should be designed such that they fulfil the natural human motion for a variety of patients. In other words, the detailed motion of human being may be different case by case because of variation in size and range of motion of each patient. However, robot should be adjustable to the new trajectory in order to provide the smoothest motion as well as avoiding physical injuries. This goal is achieved by means of translating the human motion characteristics to some biomechanical design specifications. It means that, they should be anthropomorphic and ergonomic [190].

\subsection{Robotic Design}

To design the wearable robot several parameters need to be considered such as anthropometry of the targeted users, robot weight restrictions, comfort, ergonomic, range of motion, and physical limitations. Every one of these parameters will be discussed and set in the following. 


\subsubsection{Targeted Users and Subsequent Anthropomorphic Data}

Adaptability is one of crucial factors in design. In other words, a wearable robot should be designed such that it is adaptable to the range of users with various weights, heights, and etc. Since the center of gravity and length of limbs as well as weight change as humans grow up, the probable age of users must be considered in the design process. In this section, the targeted age range of users will be determined, and this range will be used to obtain the subsequent parameters based on age.

According to research, age is a crucial factor among stroke patients. The risk of stroke doubles each 10 years after the age 55 [191]. Thus, the targeted range is set to patients between 50 to 80 years old as the most vulnerable age range for stroke. The height and weight of males from all races and ethnicities change within this age range, and were obtained as follows [192]

Table 3. 2. Weigh $(\mathrm{kg})$ of the targeted age and percentile [192]

\begin{tabular}{cccccccccccc}
\multicolumn{10}{c}{} & \multicolumn{10}{c}{ Percentile } \\
\cline { 3 - 11 } & Mean & $5^{\text {th }}$ & 10 th & 15 th & 25 th & $50^{\text {th }}$ & 75 th & 85 th & 90 th & 95 th \\
\cline { 2 - 11 } $50-59$ years & 86.0 & 63.4 & 68.2 & 72.0 & 75.7 & 84.1 & 94.0 & 100.7 & 105.3 & 114.3 \\
\hline $60-69$ years & 83.1 & 61.1 & 64.5 & 67.7 & 72.8 & 82.4 & 92.5 & 98.4 & 102.0 & 107.3 \\
\hline $70-79$ years & 79.0 & 58.5 & 62.0 & 64.2 & 68.8 & 77.9 & 87.0 & 93.5 & 96.1 & 103.3
\end{tabular}


Table 3. 3. Height (centimeter) of the targeted age and percentile [192]

\begin{tabular}{cccccccccccc} 
& \multicolumn{10}{c}{ Percentile } \\
\cline { 3 - 11 } & Mean & 5 th & 10 th & 15 th & 25 th & $50^{\text {th }}$ & 75 th & 85 th & 90 th & 95 th \\
\cline { 2 - 11 } $50-59$ years & 175.7 & 164.5 & 167.1 & 168.5 & 171.1 & 176.0 & 180.2 & 182.5 & 184.0 & 186.8 \\
\hline $60-69$ years & 174.1 & 162.1 & 165.2 & 167.3 & 169.6 & 174.3 & 179.0 & 181.4 & 183.0 & 185.1 \\
\hline $70-79$ years & 171.9 & 161.3 & 163.4 & 164.6 & 167.1 & 171.9 & 176.4 & 179.1 & 180.4 & 183.5
\end{tabular}

As mentioned in the previous tables (Table 3. 2 and Table 3.3) range of height among patients between $50-80$ years old is 62 to $114.3 \mathrm{~kg}$ and range of height is 161.3 to 186.8 centimeters. Thus, assistive robot must be adjustable for this range and must be able to provide assistive force for patients with different weights.

The first parameter evaluated was the size of the assistive robot. The crucial dimensions for the size of the robot are shown in the following table:

Table 3. 4. Critical dimensions by height range

\begin{tabular}{lcccc} 
& $\begin{array}{c}\text { Shoulder to } \\
\text { Hip }\end{array}$ & $\begin{array}{c}\text { Shoulder to } \\
\text { Shoulder }\end{array}$ & $\begin{array}{c}\text { Shoulder to } \\
\text { Elbow }\end{array}$ & $\begin{array}{c}\text { Elbow to } \\
\text { Hand }\end{array}$ \\
\cline { 2 - 5 } Dimensions as a Function of Height & $0.288 \mathrm{H}$ & $0.258 \mathrm{H}$ & $0.168 \mathrm{H}$ & $0.254 \mathrm{H}$ \\
\hline Range of the Length of the Limbs $(\mathrm{cm})$ & $46.5-53.8$ & $41.3-48.2$ & $27.1-31.4$ & $40.9-47.4$ \\
\hline$C G$ from proximal $(\mathrm{cm})$ & - & - & $11.8-13.7$ & $27.9-32.3$
\end{tabular}




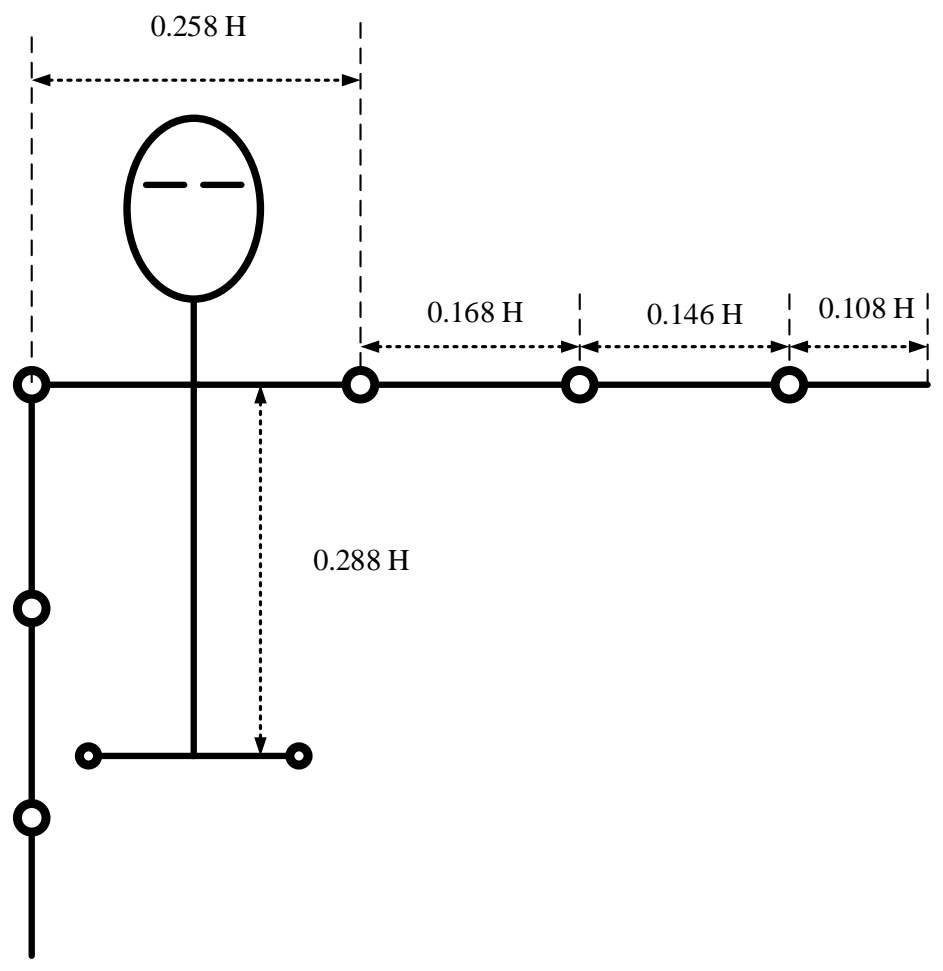

Figure 3. 9. Schematic illustration of critical dimensions by height range

The second parameter that must be considered is the weight of limbs and amount of required assistive force. Ranges for the weight, $C G$, and radius of gyration of the limbs as functions of weight were listed and the targeted ranges are shown in Table 3. 1.

Table 3. 5. Range of weight and moment of inertia of the limbs of the target range

\begin{tabular}{lcc} 
& Upper arm & Forearm and Hand \\
\cline { 2 - 3 } Weight $(\mathrm{kg})$ & $1.7360-3.2004$ & $1.3640-2.5146$ \\
\hline Moment of Inertia about $C G\left(\mathrm{~kg} \cdot \mathrm{m}^{2}\right)$ & $0.0132-0.0327$ & $0.0501-0.1240$
\end{tabular}

Since it is difficult to test the performance of the robot using a real human at the first stage of design, properties of the targeted age are captured and replaced with a number of masses, which simulate the moment of inertia and weight of a real human being. The properties of the target are 
provided in Table 3. 6. Since it was intended to provide only $20 \%$ assistive force, the corresponding properties of a $20 \%$ model were obtained and shown in Table 3. 6 . This $20 \%$-model was a number of masses put together such that they simulated $20 \%$ of the masses of limbs. The moment of inertia was mentioned before as a function of mass and radius of gyration of limbs. Thus, applying the $20 \%$ of the masses of the actual limbs and using the radius of gyration of the corresponding limbs, moment of inertial was obtained and shown in Table 3. 6.

It was expected that the robot provides enough force to perform the ADL if the simulated model is only $20 \%$ of the real upper extremities.

Table 3. 6. Physical properties of the $20 \%$ model

\begin{tabular}{lcc} 
& Upper arm & Lower arm and Hand \\
\cline { 2 - 3 } Mass $(\mathrm{kg})$ & $0.3472-0.6401$ & $0.2728-0.5029$ \\
\hline Moment of Inertia about $C G\left(\mathrm{~kg} \cdot \mathrm{m}^{2}\right)$ & $0.0026-0.0065$ & $0.01-0.0248$
\end{tabular}

\subsubsection{One-DOF Elbow Assistive Prototype}

To design a proper robot for assistive purposes several criteria were considered including:

- The device must be light weight but not bulky so that it can be highly portable;

- It must be wearable without special assistance; and

- The mechanism must be easily fabricated and must require minimal maintenance.

To satisfy these criteria, a one-DOF elbow assistive robot was designed and fabricated. Figure

3. 10 demonstrates the prototype of the assistive device. The prototype mechanism was made of ABS plastic using a rapid prototyping machine. To reduce the weight and increase the sturdiness of the structure, honeycomb meshing was employed for internal structure. Two set of straps around 
the forearm and one strap around the upper arm hold the mechanism on the upper extremities around the elbow joint. Due to importance of safety, two mechanical breaks for full flexion and full extension were included.

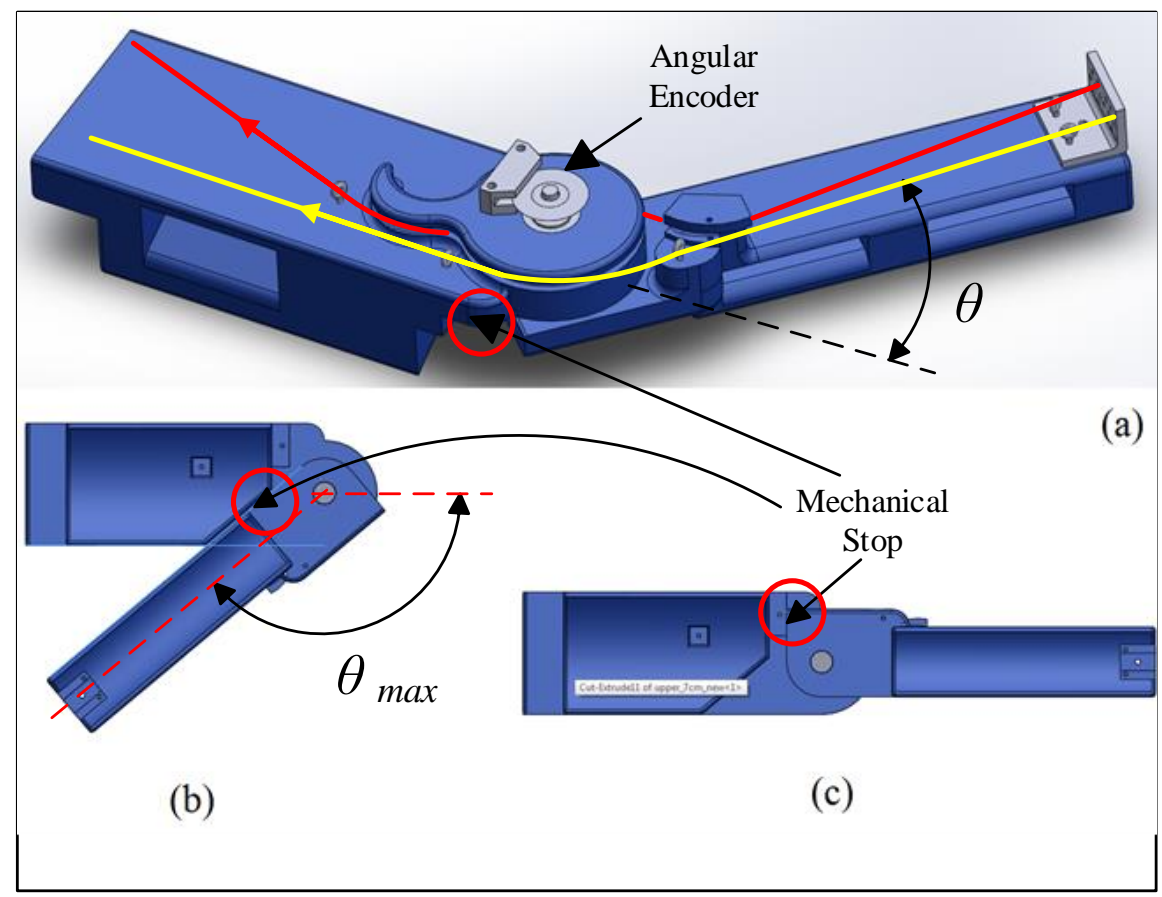

Figure 3. 10. (a) Solidworks model of the structure of the robot (b) Full flexion mechanical stop (c) Full extension mechanical stop

Two sets of strands were employed to provide extension and flexion motions for the forearm. One end of the twisted strings is connected to the motor shaft and the length of string can be adjusted according to the height of patient and the applicable linear range of motion. Several specifications, such as geometric limits and power constraints were considered while designing the mechanism.

For the geometric limits, the radius of the pulley on the elbow joint can greatly affect the output torque. With the relationship between torque and force $\tau=r \cdot F$, it is clear that greater $r$ can provide more torque if the output force remains the same. However, large $r$ can yield a bulky 
mechanism design and increase the weight. A design diameter of $52 \mathrm{~mm}$ was chosen for the pulley as a reasonable compromise.

The range of flexion angle $(\theta)$ varies in different textbooks from is $146^{\circ}$ [193] to $135^{\circ}$ [166] [167] depending on the subject of experiment. In this prototype it is assumed the maximum angle is $146^{\circ}$. It should be noted that the range of the elbow angle $\theta$ varies in different ages. Thus, in order to avoid further injuries due to over twist of the joint, the mechanical stop, shown in Figure 3. 10, should be adjusted accordingly. With these two given parameters, $r$ and angle $\theta_{\max }$, the maximum linear actuation required by the twisted string mechanism is achieved $66.2 \mathrm{~mm}$.
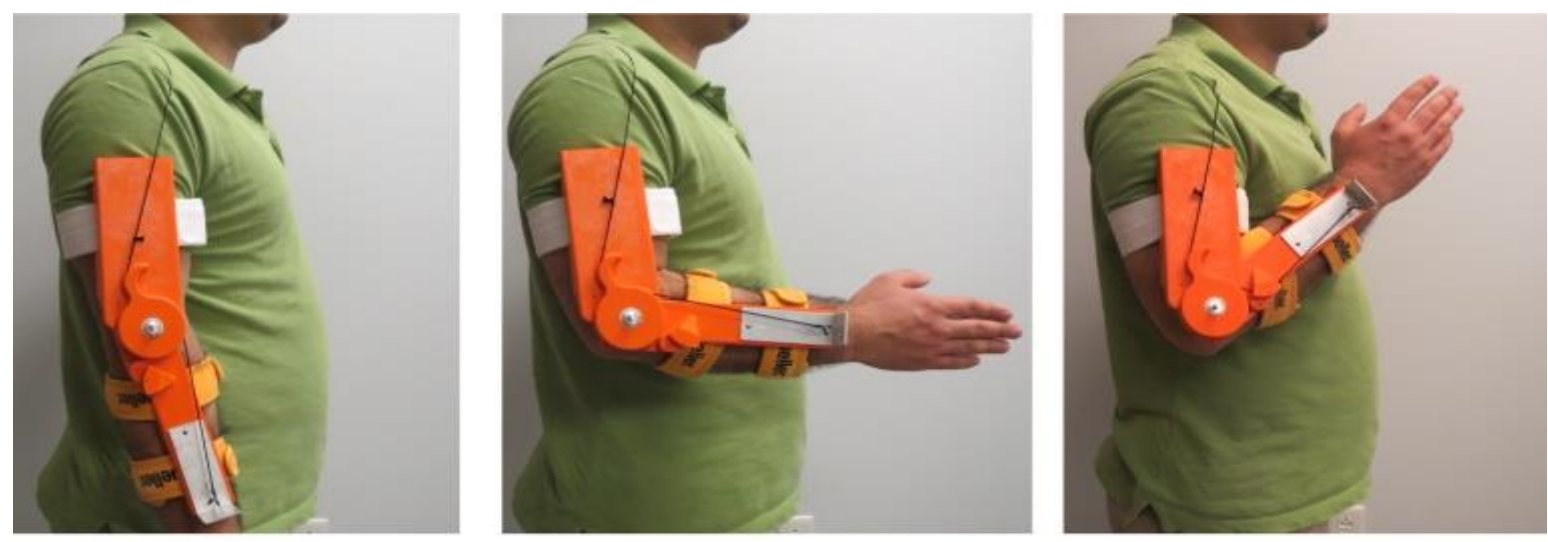

Figure 3. 11. Prototype mounted on the upper extremities in different positions

\subsubsection{Four-DOF Wearable Robot}

The scope of this section is designing a four DOF robot for shoulder and elbow assistance and then to propose a wearable robot for the backpack.

\section{Robotic Arm}

In this design two joints were actuated, one for elbow and one for shoulder, and two DC motors were assigned to each joint, one for flexion and one for extension. To reduce the weight of the 
assistive robot, the actuators were set on the wearable back pack and the force was applied to the place of actuation through Bowden cables, commonly called bicycle cables.

The first step was to estimate the maximum assistive force required to perform tasks. For this purpose Newton's equations was used with steady state assumption, with the result that the corresponding acceleration forces were negligible since most of tasks were quite slow. This assumption provided a good approximation of how much force would be needed in different phases. However, a kinetic and a kinematic model (in appendix) were developed in order to predict the exact amount of dynamic torque for every ADL.

The model of the upper extremities is shown below.

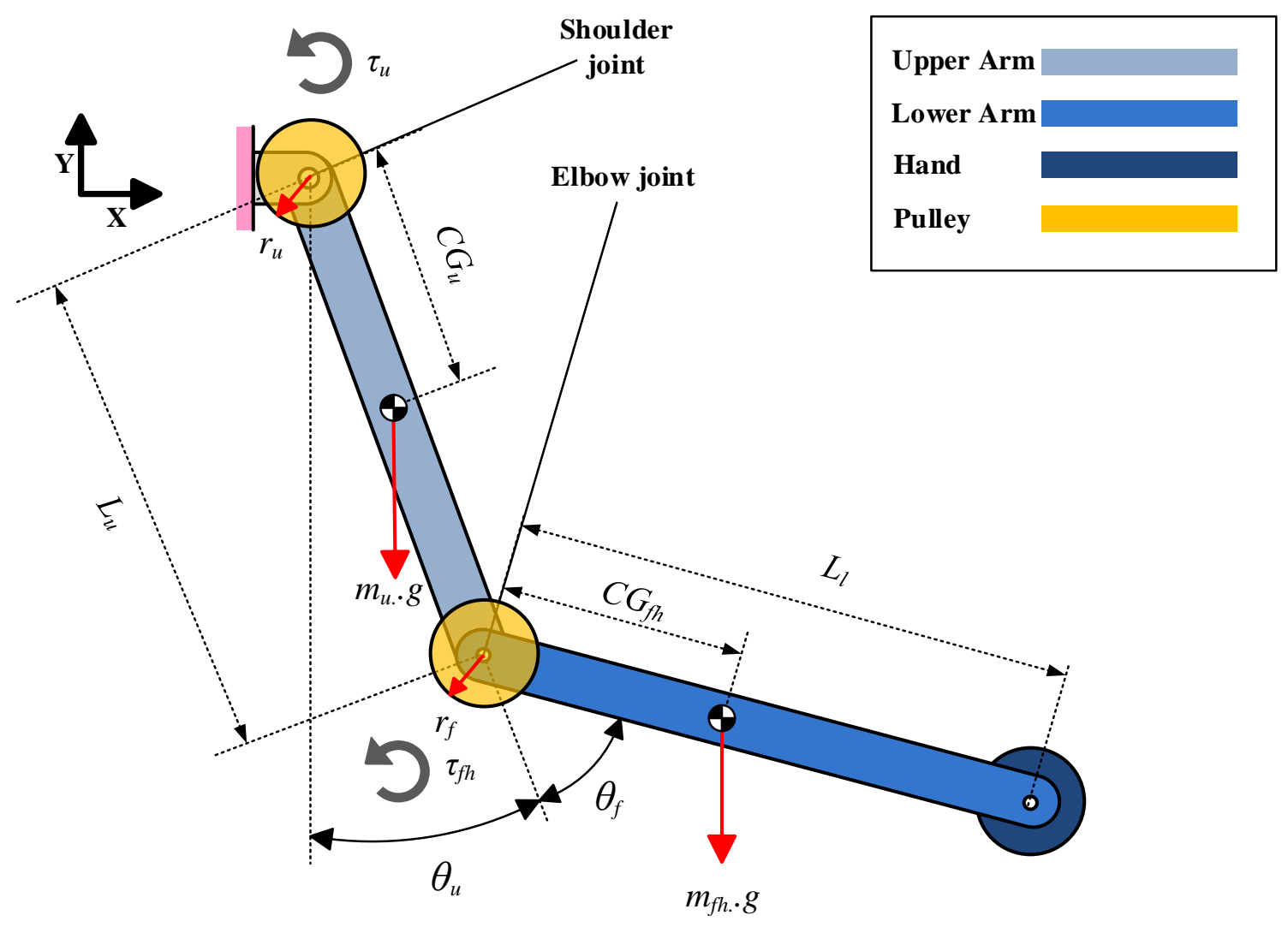

Figure 3. 12. Schematic of the upper extremities with driving torques

where the parameters are shown in Table 3. 7. 
Table 3. 7. Parameters of upper extremities

\begin{tabular}{ll|ll}
$m_{u} \quad$ Upper arm mass & $C G_{u} \quad$ Distance of the upper arm $C G$ from proximal \\
\hline$m_{f} \quad$ Forearm mass & $C G_{f h} \quad$ Distance of the forearm and hand $C G$ from proximal \\
\hline$m_{h} \quad$ Hand mass & $C G_{u} \quad$ Distance of the upper arm $C G$ from proximal \\
\hline$\theta_{u} \quad$ Upper arm angle & $\tau_{u} \quad$ Required torque at shoulder to keep it stationary \\
\hline$\theta_{f} \quad$ Forearm angle & $\tau_{f h} \quad$ Required torque at elbow to keep it stationary \\
\hline$L_{u} \quad$ Upper arm segment length & $L_{f} \quad$ Forearm segment length from elbow to hand $C G$ \\
\hline$r_{u} \quad$ Pulley radius at shoulder & $F_{u} \quad$ Corresponding axial force to $\tau_{u}$. \\
\hline$r_{f} \quad$ Pulley radius at elbow & $F_{f h} \quad$ Corresponding axial force to $\tau_{f}$
\end{tabular}

Applying Newton's first law and assuming the elbow joint is stationary $F_{f h}$, which is the corresponding axial force to $\tau_{f h}$ will be

$$
\begin{gathered}
\tau_{f h}=C G_{f h} m_{f h} g \cos \left(\theta_{f}+\theta_{u}\right), \\
F_{f h}=\left(C G_{f h} m_{f h} g \cos \left(\theta_{f}+\theta_{u}\right)\right) / r_{f},
\end{gathered}
$$

Applying Newton's First Law to the shoulder joint, the required force to keep the upper arm stationary would be:

$$
F_{i u}=\left(m_{u} g C G_{u} \cos \left(\theta_{u}\right)+\tau_{f h}\right) / r_{u},
$$

The primary force required was to overcome gravitational acceleration, and thus the maximum force was required when the elbow flexion was $0^{\circ}$ and shoulder flexion was $90^{\circ}$. This position is shown schematically below. 


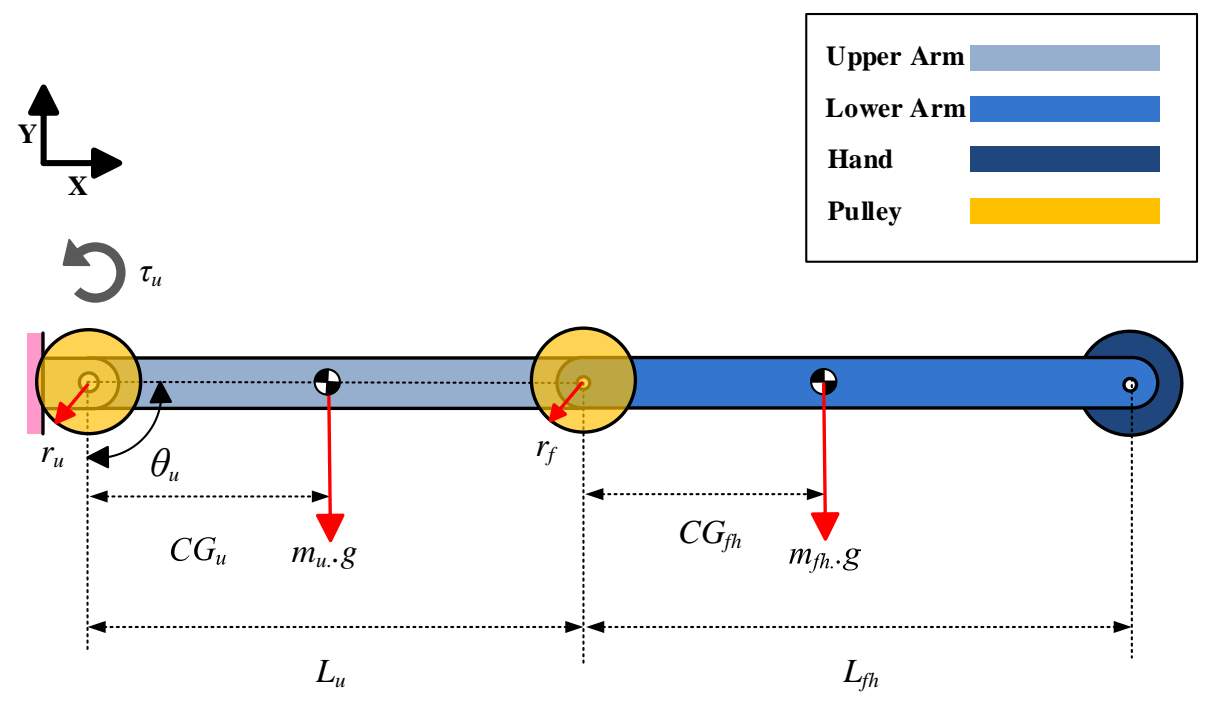

Figure 3. 13. Schematic of the upper extrmity in maximum required torque position

Applying the maximum torque position, the maximum axial forces will be

$$
\begin{gathered}
F_{i f h}=\left(C G_{f h} m_{f h} g\right) / r_{f}, \\
F_{i u}=\left(m_{u} g C G_{u}+\tau_{f h}\right) / r_{u},
\end{gathered}
$$

As shown in (3.3) and (3.4), the bigger $r_{u}$ and $r_{f}$ are, the less force is required to control the arm. On the other hand there are physical limitations for the size of the pulley in order to avoid bulkiness of the arm robot. According to the average shoulder and elbow size, two pulleys, one with 2 inches diameter (for small size patients) and one with 3 inches diameters (for normal size patients) were selected. However, only the second one was used. Using Table 3. 6, and (3. 3) and (3. 4) the range of the axial force will be:

Table 3. 8. Static forces needed to maintain the arm stationary

\begin{tabular}{cc}
$F_{u}(\mathrm{~N})$ & $F_{f h}(\mathrm{~N})$ \\
\hline $30.1460-64.4037$ & $19.5971-41.8243$
\end{tabular}


This table was eventually used to determine the axial force on strings and consequently the behavior of the cable under different axial forces and the type of DC motor, which could still perform under such axial force.

To select the pulley, the static forces as well as the material of the transmission cable was considered. The force was transmitted through a bicycle brake cable. Thus, a heavy duty nylon pulley with 3 inches diameter and 800 lbs. work load limit was selected. Solidworks model of the designed robot structure is shown in Figure 3. 14.
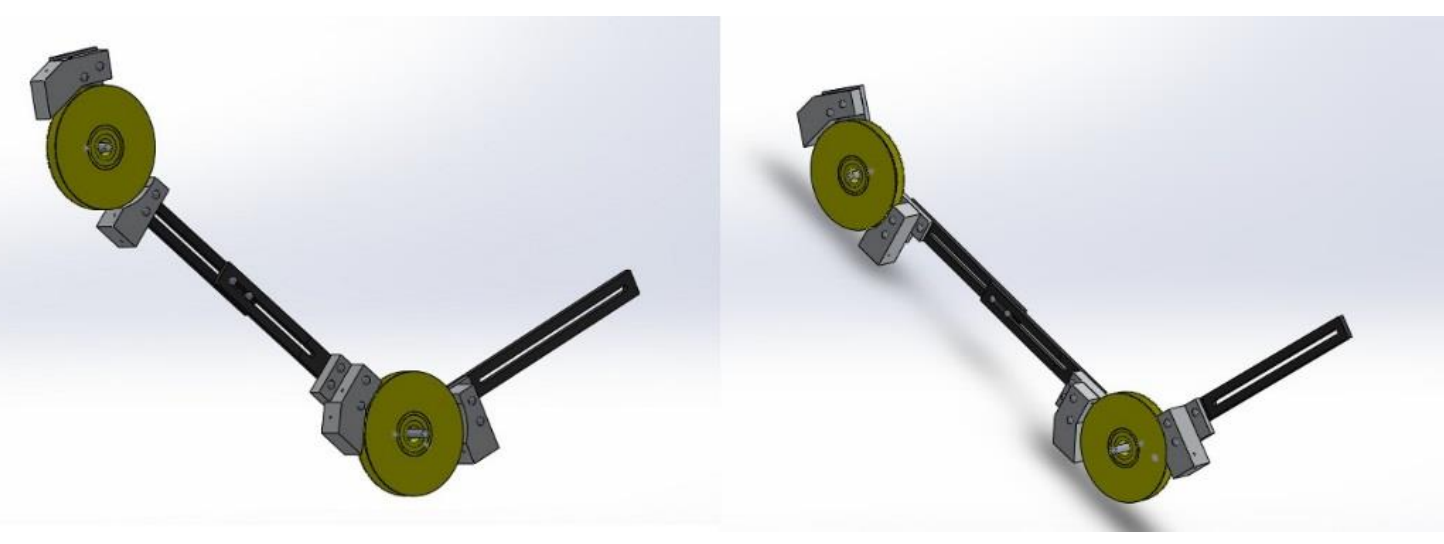

Figure 3. 14. Solidworks model of the robotic hand

Two Shimano Bowden cables were used for each joint, one for flexion and one for extension whose directions are shown below.

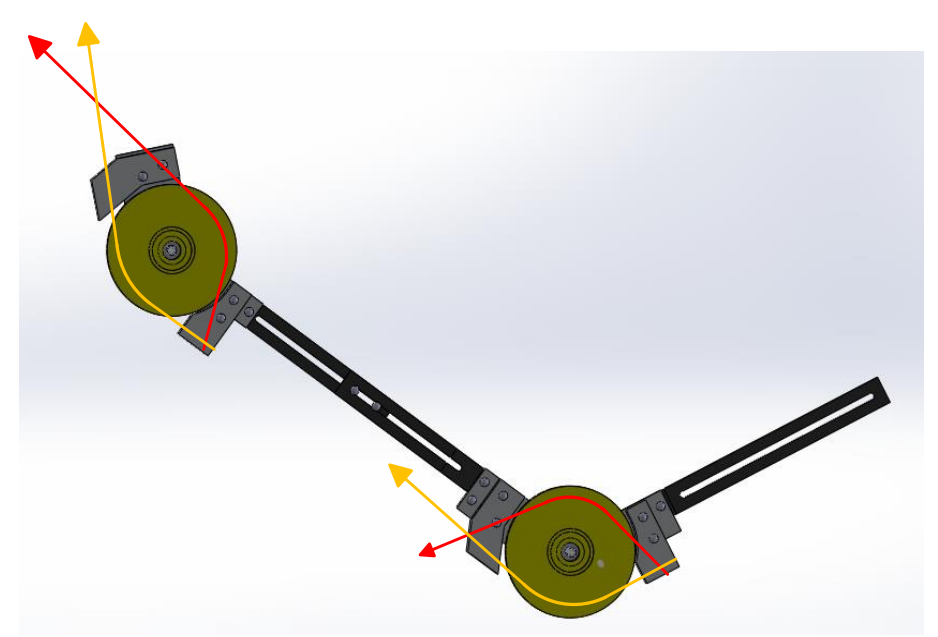


Figure 3. 15. Direction of the actuating cables

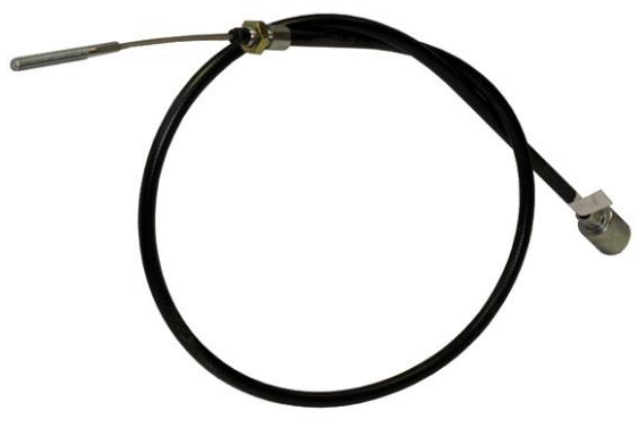

Figure 3. 16. Bowden cable [194]

Considering the maximum flexion/extension of the shoulder and elbow joints and the useful range of motion that must be provided, the maximum values for $\theta_{u}$ and $\theta_{f}$ were set to $120^{\circ}$ and $135^{\circ}$ respectively. Moreover, the size of the components for the upper arm and forearm were set such that they were adaptable to the range of the target user dimensions.

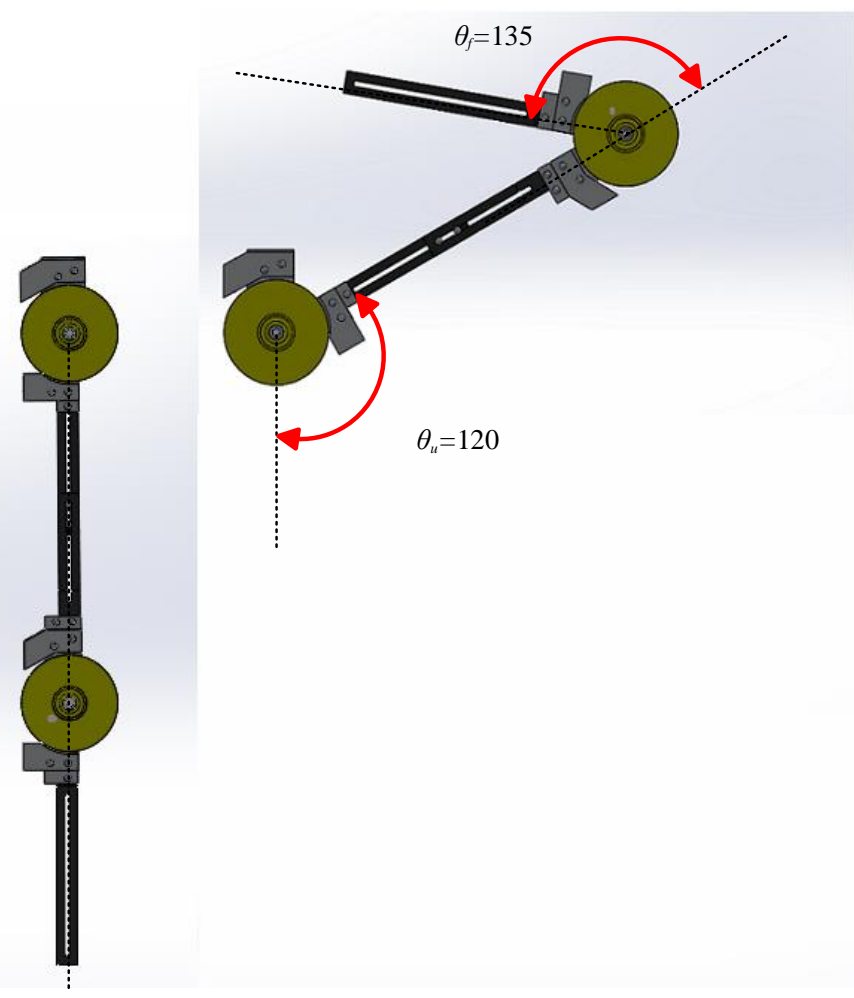

Figure 3. 17. Range of motion for flexion and extension of shoulder and elbow joints 
The radius of the pulley was selected to be 3 inches diameters. Thus, the range of axial contraction to provide full flexion and extension for shoulder and elbow became

Table 3. 9. Shoulder and elbow axial contraction

\begin{tabular}{cc} 
Shoulder Contraction $(\mathrm{cm})$ & Elbow Contraction $(\mathrm{cm})$ \\
\hline 7.97 & 8.97
\end{tabular}

The mechanical properties of the arm for simulation are provided in Table 3. 10.

Table 3. 10. Properties of the robotic hand

\begin{tabular}{lcccc} 
& Mass $(\mathrm{kg})$ & Length $(\mathrm{cm})$ & $C G(\mathrm{~m})$ from proximal & I (kg/m2) at $C G$ \\
\cline { 2 - 5 } Shoulder to elbow & 0.434 & $27.1-31.4$ & $0.147-0.171$ & $0.005305-0.007356$ \\
\hline Elbow to hand & 0.131 & - & 0.074 & 0.000474
\end{tabular}

In order to attach the robotic arm comfortably to the upper arm and forearm, a nylon cushion similar to the shape of the forearm and upper arm was designed. This piece fits between the human arm and the robot arm and prevents direct contact between them. Moreover, to keep the arm almost rigidly attached to the robot arm, special pieces were designed to hold the arm and keep it attached by straps.

The robotic arm with the arm holder is shown in Figure 3. 18. A computer model of a real human being was used to show how it can be fitted to a human arm. 

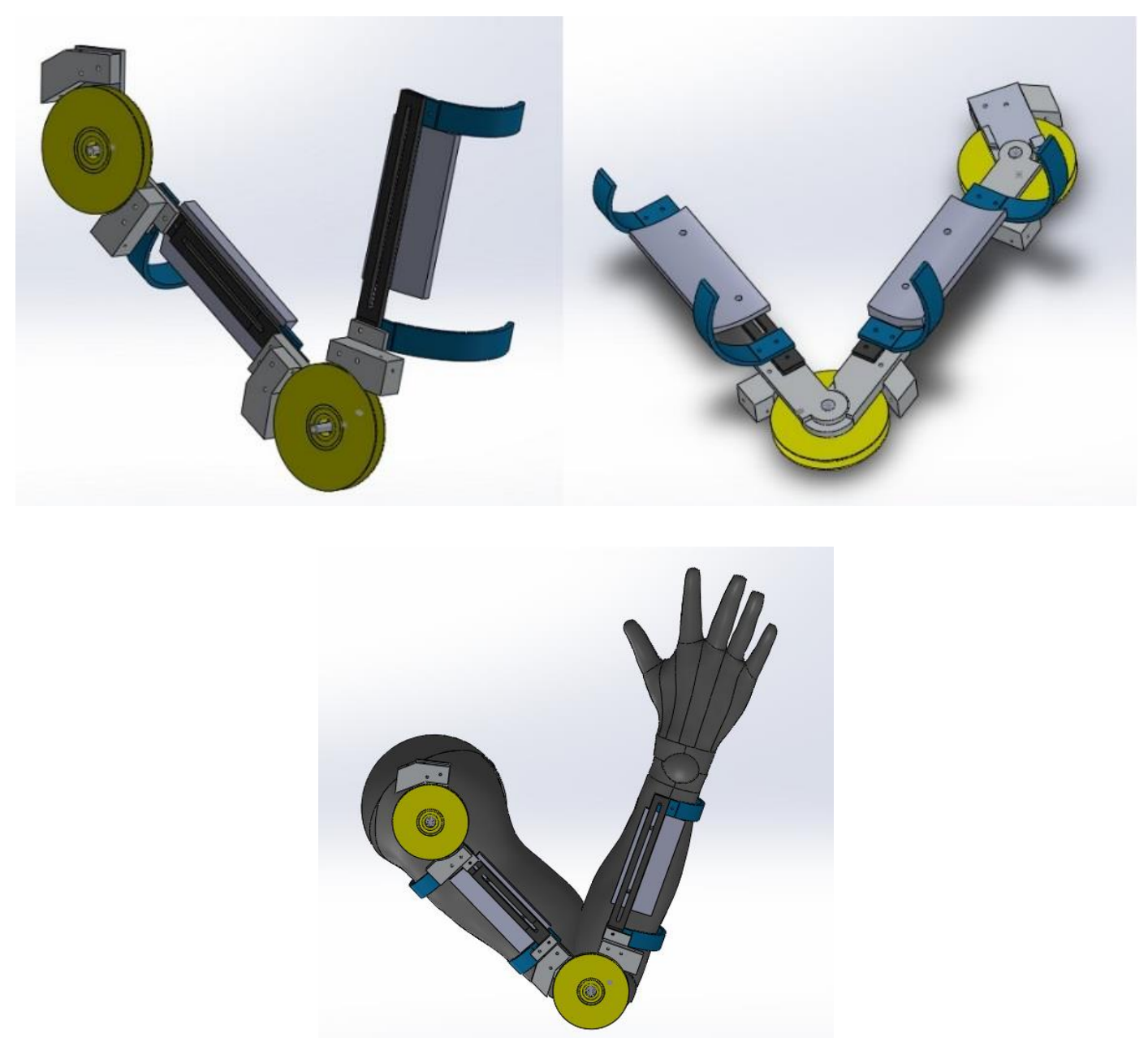

Figure 3. 18. (Top) Schematic of the arm holders and (bottom) schematic of the arm robot on a modeled human upper extremities

\section{Backpack Component}

The arm mechanism must be mounted on a fixed stand. In this case, the fixed stand was a wearable backpack, where all equipment such as the DC motors, the actuation system, and processing unit will be mounted.

Weight, adaptability, and ergonomics of the backpack were the major issues to be considered in the design process [195] [196]. According to these factors, a light weigh adaptable backpack was designed, which is shown below. To show the adaptability and compatibility of the robot, it was mounted on a model of a real human being. 

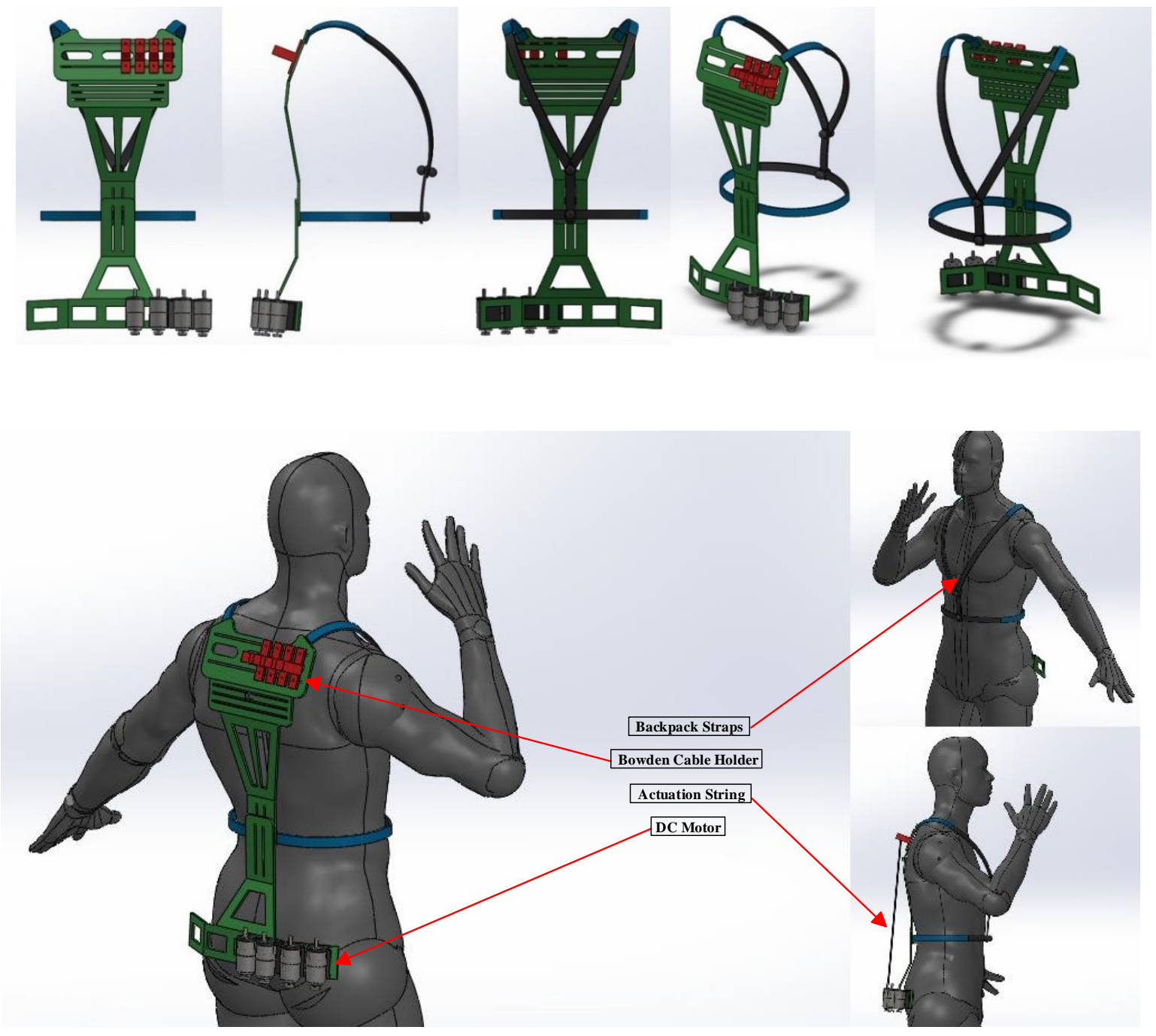

Figure 3. 19. Backpack design and how it is set on human

The backpack had two parts: one for the lower lumbar, where DC motors were mounted on and one for the upper lumbar, where Bowden cable holders were mounted on (Figure 3. 19). These two piece could slide over each other to be adjusted for different patients with different heights.

\section{Shoulder Complex Design}

There are two major issues with the design of an exoskeleton shoulder joint, which can fulfill the natural motion of the human shoulder joint:,

- How to articulate the translation of the shoulder complex due to the motion of the scapula; 
- Where to put the singularities of the shoulder joint to minimize the interruption in the natural flow of motion.

Designing a robotic shoulder joint with 11 or more degrees of freedom was unreasonable for a lightweight, low-cost mechanism. At most, 3 degrees of freedom seem reasonable, with the motions being rotations about the three principal axes. The $(\mathrm{GH})$ joint is kinematically modeled as a ball and socket joint. However, implementation of the same type of joint in an exoskeleton robot is not only challenging but also results in interference between the human joint and the robotic joint. Therefore, the robotic joint is often fabricated by using revolute joints connected in series. However, this solution introduces two singularities in which the robotic arm can lose one degree of freedom if it is trapped.

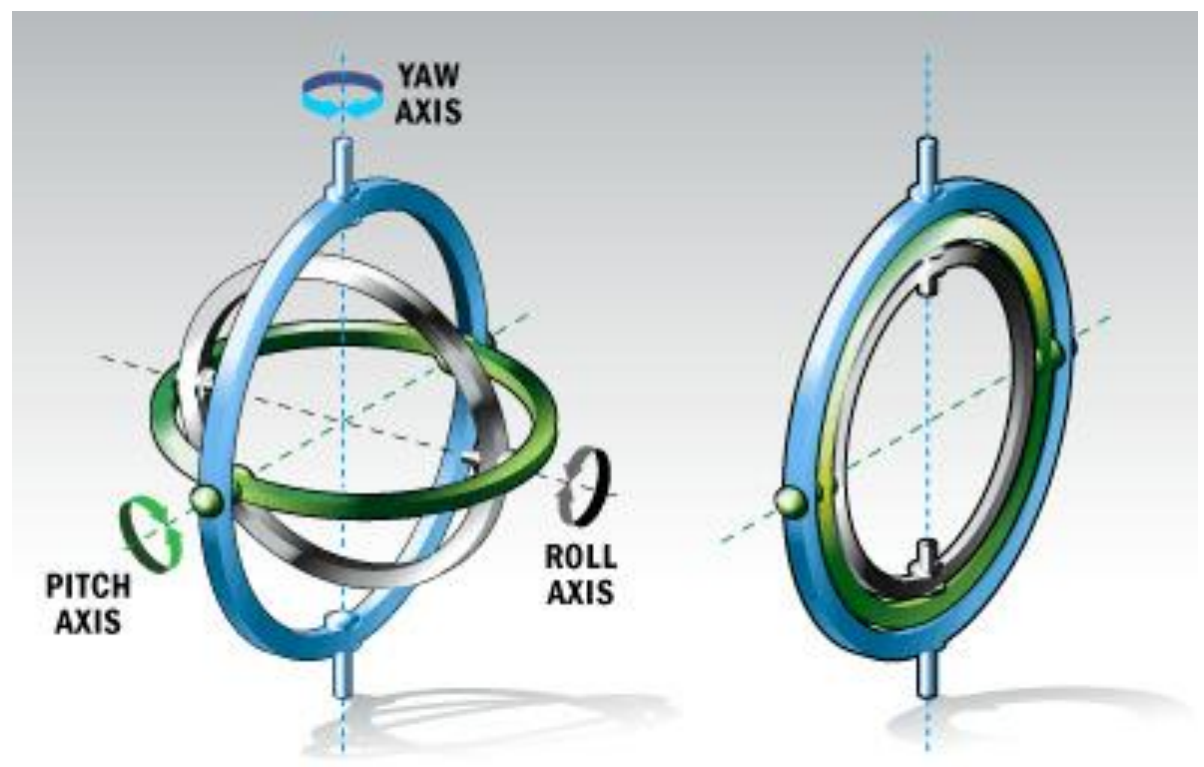

Figure 3.20: (Left) three axes of rotation in non-singular condition (right) in gimbal lock [197]

These singularities, called gimbal lock, can be relocated but not removed and scientists attempt to put them on an axis, which is unlikely to interrupt the desired motion for the application. Carignan et al. [158] investigated potential combinations of the serial joints for a shoulder rehabilitation exoskeleton. They modeled the shoulder joints with four degrees of freedom such 
that they capture the biggest workspace for the desired task while avoiding singularities. Figure 3. 21 shows two configurations of joints one with a singularity in the working range and one without.
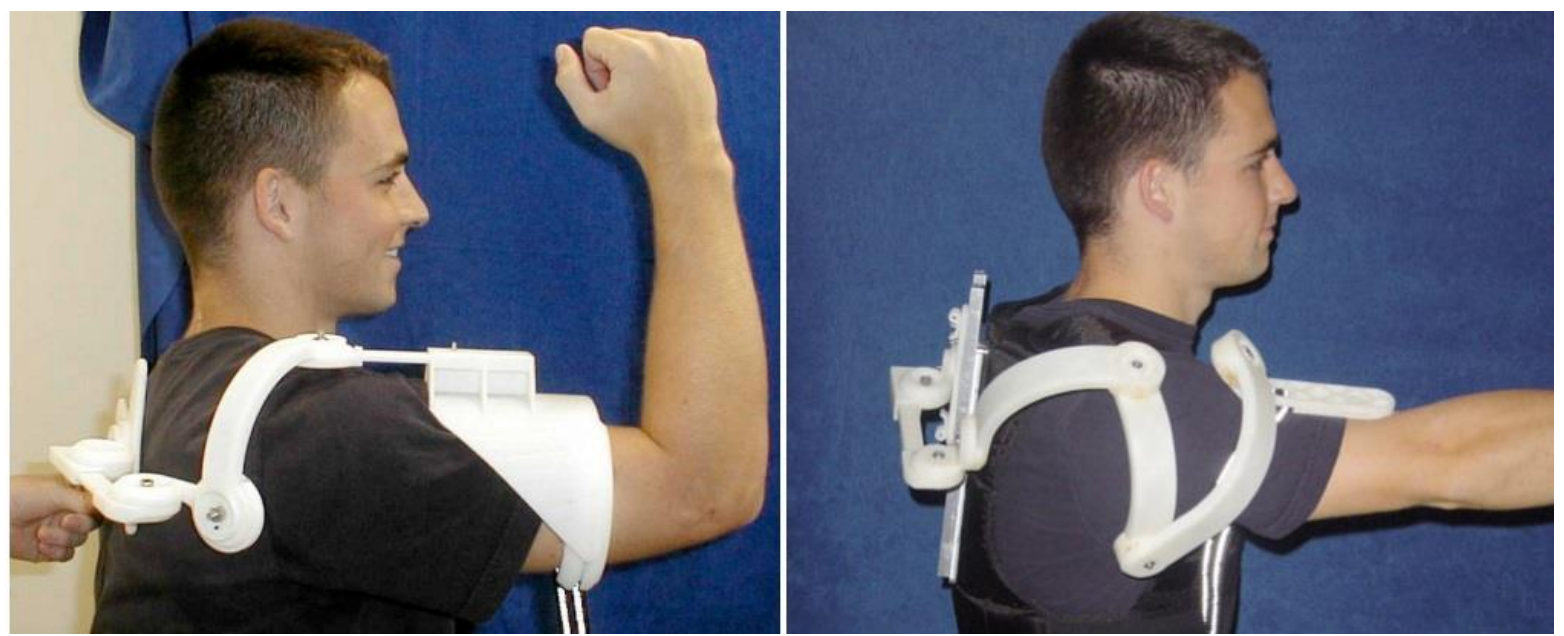

Figure 3. 21. Two rapid prototypes of the shoulder rehabilitation exoskeleton with (left) singular combination of joints and (left) non-singular combination. Photos reproduced from reference [158]

As shown in the left side of Figure 3. 21, the shoulder joint will have a singularity if the first and third rotational joints are parallel to the transverse plane. However, by changing the configuration of joints and adding an extra degree of freedom with an angle relative to the robotic arm, the singularity was relocated to a position outside the range of motion and the shoulder joint was enabled to fulfil with the natural motion of human shoulder joint [158].

The ranges of motion of joints for various ADLs were previously studied by many researchers, including [198] [199] [200]. The purpose of this section is to design a shoulder joint that is able to cover the range of motion for necessary ADLs such as eating, pouring water, drinking, and etc. The range of motion for certain ADLs are listed in the following.

A new configuration of joints with three degrees of freedom for the shoulder was inspired from the work of Carignan [158] specifically for eating and drinking. In this design one degree of freedom was sacrificed for the sake of lighter weight and more compact design. The singularity was set outside the range of motion for these ADLs. The sequence of three pinned joints was such 
that the first two were orthogonal and independent of each other. The first pinned joint was along the transverse axis and parallel to the Sagittal and Transverse planes.

Table 3. 11. Range of motion of shoulder and elbow joints according to the ISB standard ${ }^{1}$ [201] [198]

\begin{tabular}{lcccc|cc} 
& \multicolumn{3}{c}{ Shoulder (thoracohumeral) join } & \multicolumn{2}{c}{ Elbow joint } \\
\cline { 2 - 7 } & Elevation & $\begin{array}{c}\text { Plane of } \\
\text { elevation }\end{array}$ & $\begin{array}{c}\text { ISBAR } \\
\text { axial } \\
\text { rotation }\end{array}$ & $\begin{array}{c}\text { NSAR } \\
\text { axial } \\
\text { rotation }\end{array}$ & Flexion & $\begin{array}{c}\text { Forearm } \\
\text { rotation }\end{array}$ \\
\cline { 2 - 7 } Washing the face & 44 & 111 & -57 & 17 & 128 & 69 \\
\hline Eating with a spoon & 56 & 74 & -49 & -12 & 123 & 1 \\
\hline $\begin{array}{l}\text { Pouring water into a } \\
\text { glass }\end{array}$ & 64 & 46 & -17 & -1 & 93 & 146 \\
\hline Drinking with a glass & 87 & 80 & -62 & -60 & 115 & 110
\end{tabular}

The third one, however, was skewed by $30^{\circ}$ relative to the second consecutive joint. Thus, the third joint could be described as a function of three orthogonal joints, with two of them being used here as the first and second pinned joints.

The shoulder joint provided $60^{\circ}$ shoulder elevation and $90^{\circ}$ plane elevation. Elevation angles on planes other than the frontal depended on the angle of abduction/adduction $\left(120^{\circ}\right)$ provided by the third pinned joint of the shoulder complex. If the upper arm and the forearm were placed parallel to the sagittal plane, the full $120^{\circ}$ of shoulder elevation was possible.

\footnotetext{
1 "For the shoulder elevation angle, the limb position when the upper arm coincided with the frontal and sagittal axes was defined as $0^{\circ}$. For the plane of elevation, the limb position in which the upper arm coincided with the frontal plane was defined as $0^{\circ}$ and that when the upper arm coincided with the sagittal plane was defined as $90^{\circ}$. A positive plane of elevation means horizontal flexion; a negative plane of elevation means horizontal extension. Elevation takes only positive values; therefore, it has been determined whether forward elevation or backward elevation was present by looking at the sign for the plane of elevation. ISBAR is always 0 when the forearm is directed horizontally. In $N S A R, O^{\circ}$ was defined at an upper arm elevation of $0^{\circ}$ as the limb position in which the forearm coincides with the sagittal plane at $90^{\circ}$ of elbow flexion, and at an elevation of $90^{\circ}$ as the limb position in which the forearm coincides with the horizontal plane. Internal shoulder rotation is displayed as plus, and external rotation as minus. Forearm axial rotation angle was defined as $0^{\circ}$ in the limb position with $90^{\circ}$ of external rotation from the neutral position, with internal rotation displayed as plus and external rotation as minus. " [55]
} 
(a)

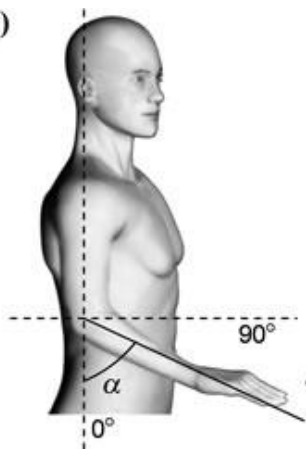

(b)

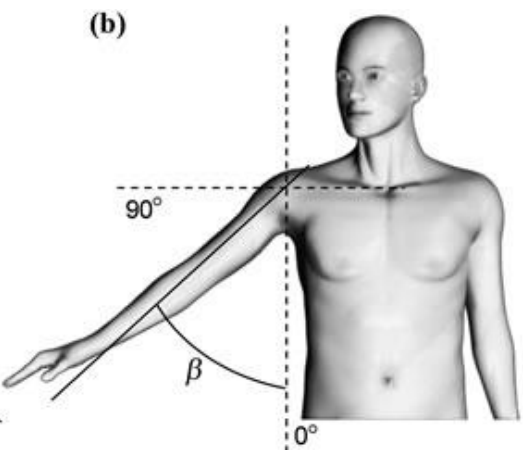

(c)

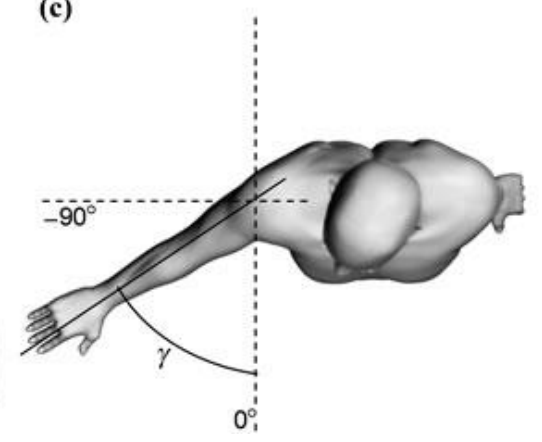

Figure 3. 22. Representations of angles at the elbow and shoulder joints in accordance with recommendations of International Society of Biomechanics (ISB): (a) elbow flexion ( $\alpha$ ), (b) shoulder elevation $(\beta)$, and (c) plane of elevation $(\gamma)$. [202]

Shoulder complex consists of five link as shown in Figure 3. 23.
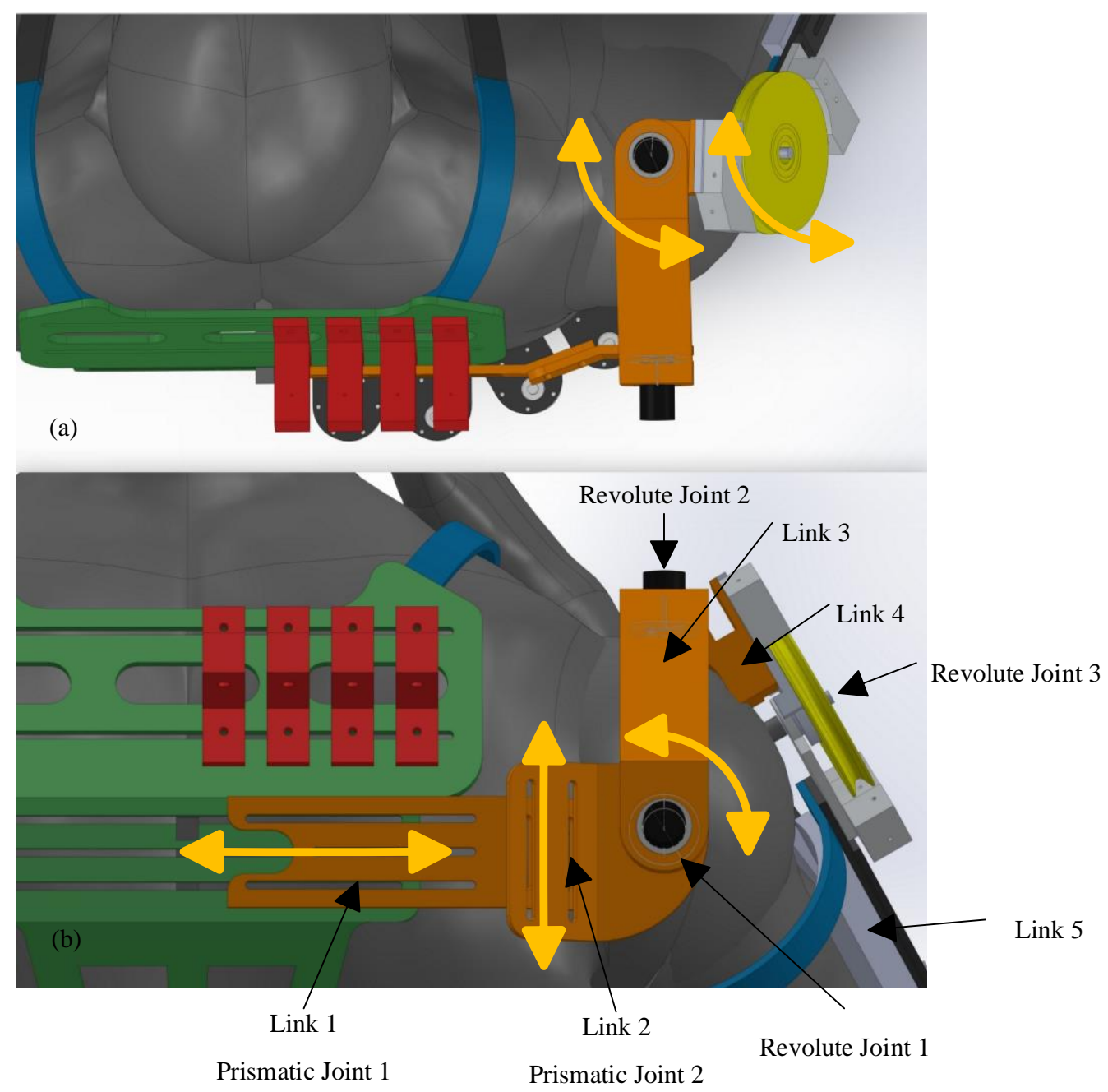

Figure 3. 23. Five links of the shoulder complex from (a) top view (b) back view 
The first two links (link 1 and 2) were prismatic joints (prismatic joint 1 and 2) and they were fixed on the backpack platform. These two links were just for the sake of adjustments and depending on where the patient's shoulder joint was located, these two joint would be adjusted accordingly. Moreover, these two link were designed according to the anthropomorphic data. Thus they could be adjusted for the targeted patients. The second two links (link 3 and 4) were revolute joints (revolute joint 1 and 2) and they provided passive motion. It should be noted that these two link were costume designed. Thus they were unique for each person. The last link (link 5) was the only revolute joint (revolute joint 3), which was actively controlled (Figure 3. 23).

\section{Summary}

In this section all three parts of the design were integrated and shown as one unit. For ease of understanding the dynamics of the problem, three parameters $\psi_{1}, \psi_{2}$, and $\psi_{3}$ as the three consequential articulations of the shoulder joint were defined, where only $\psi_{3}$ was powered, and one last parameter $\psi_{4}$ as the elbow elevation angle. The range of motion of each articulation is defined below.

Table 3. 12. Range of motion of articulations

\begin{tabular}{lllll} 
& $\psi_{1}$ & $\psi_{2}$ & $\psi_{3}$ & $\psi_{4}$ \\
\hline Range of motion & $60^{\circ}$ & $90^{\circ}$ & $120^{\circ}$ & $145^{\circ}$
\end{tabular}

Shoulder joint was designed and put on a human model to show the range of motion that it could provide. The following set of figures shows the sequence of different phases for drinking or eating and the feasibility of the action. 

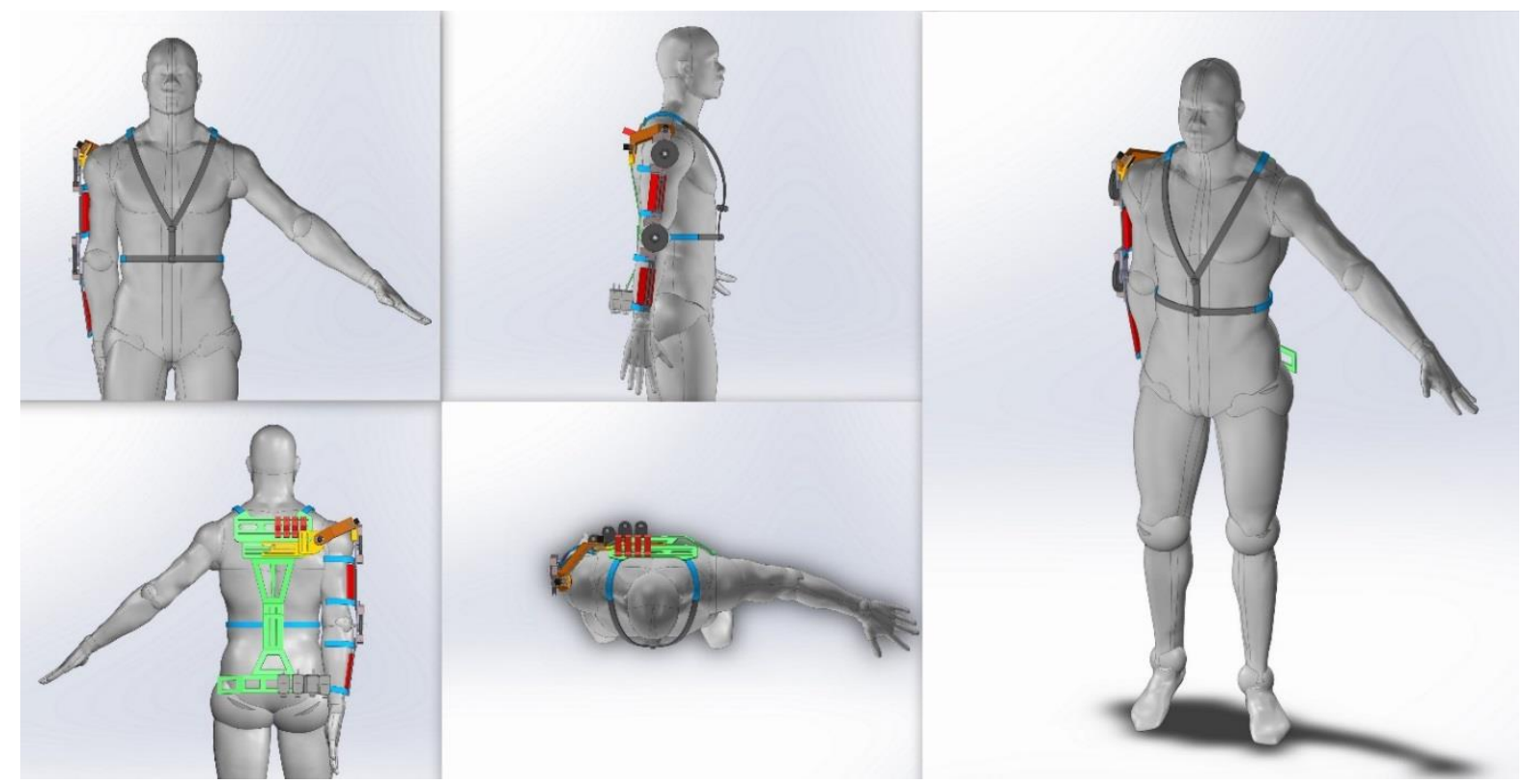

Figure 3. 24. The position of limbs when $\psi_{1}=0^{\circ}, \psi_{2}=0^{\circ}, \psi_{3}=0^{\circ}$, and $\psi_{4}=0^{\circ}$, where the upper arm and forearm are are standing parallel to the Sagittal and Frontal planes
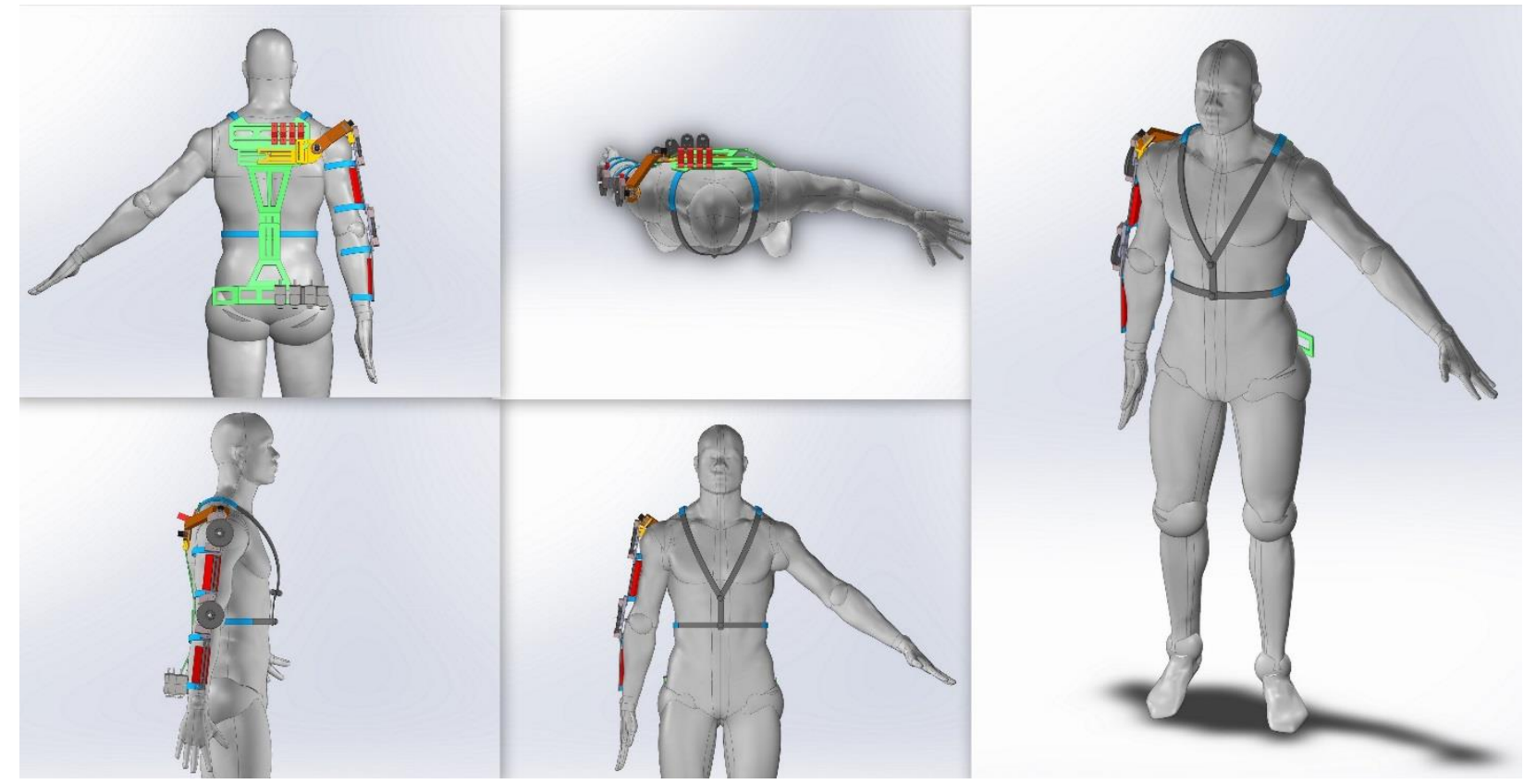

Figure 3. 25. The position of limbs when $\psi_{1}=10^{\circ}, \psi_{2}=0^{\circ}, \psi_{3}=0^{\circ}$, and $\psi_{4}=0^{\circ}$, where the upper arm and forearm are are only parallel to the Frontal plane 


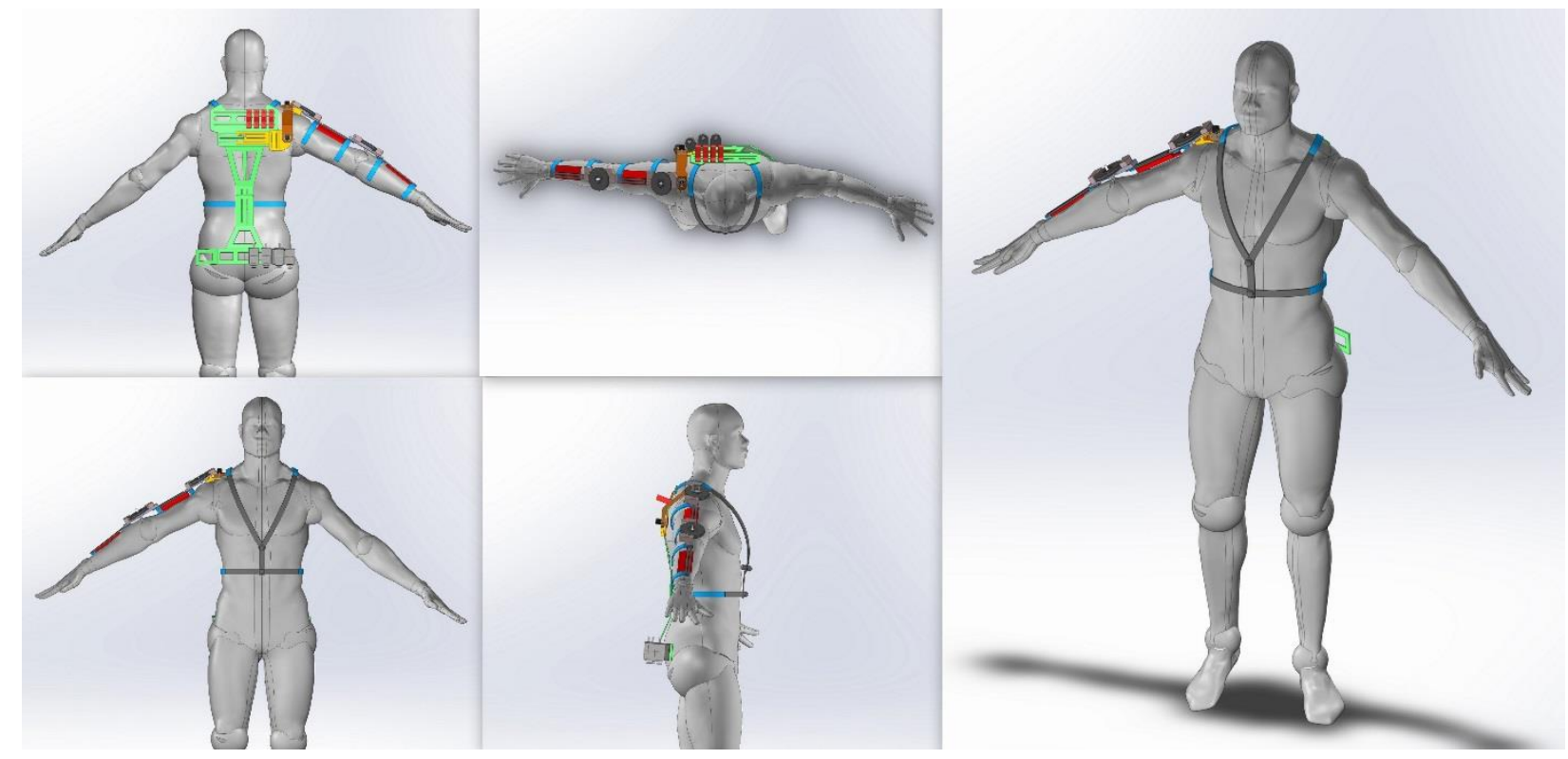

Figure 3. 26. The position of limbs when $\psi_{1}=60^{\circ}, \psi_{2}=0^{\circ}, \psi_{3}=0^{\circ}$, and $\psi_{4}=0^{\circ}$, where the upper arm and forearm are are only parallel to the Frontal plane, and $\psi_{l}$ has the maximum angle
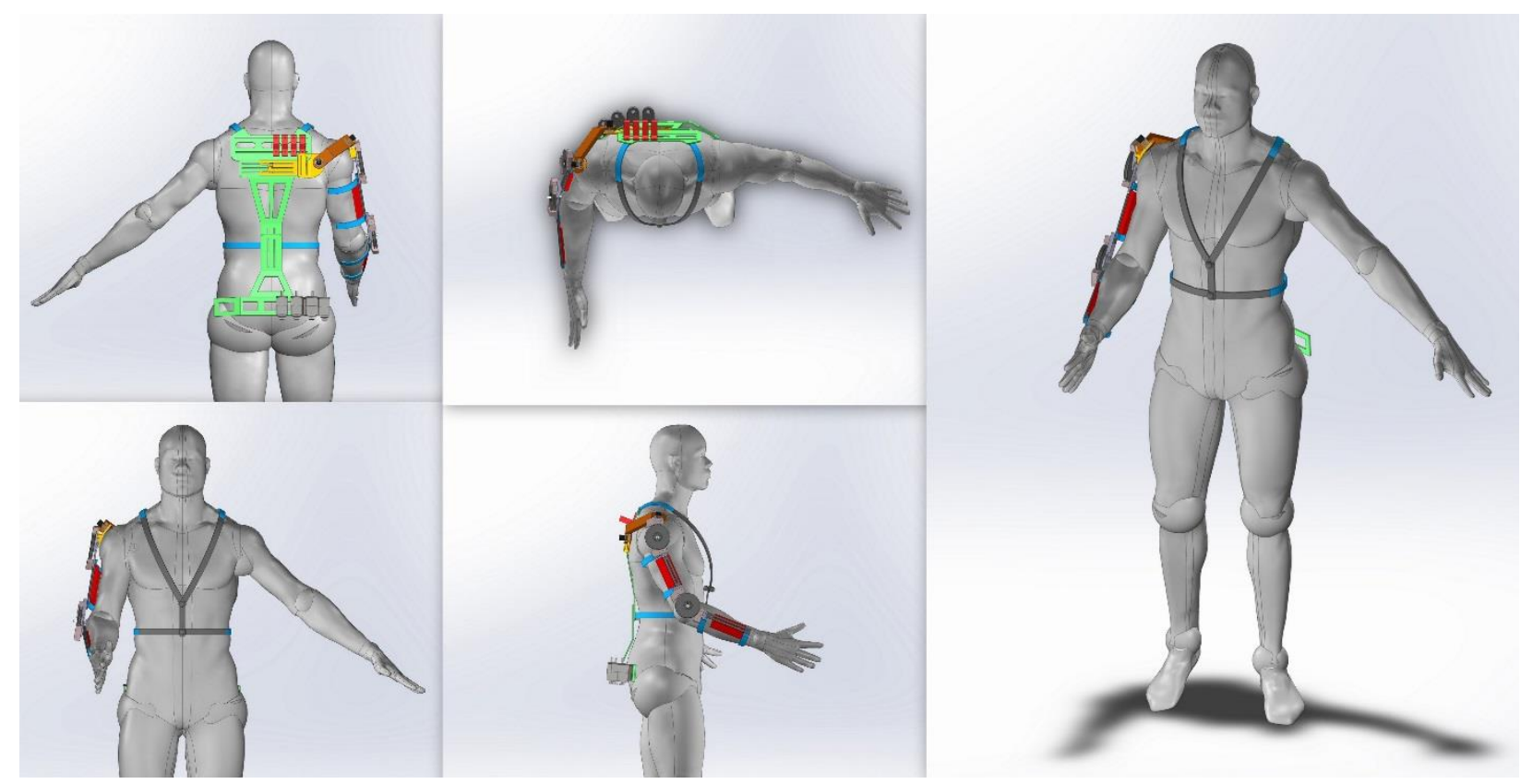

Figure 3. 27. The position of limbs when $\psi_{1}=10^{\circ}, \psi_{2}=0^{\circ}, \psi_{3}=30^{\circ}$, and $\psi_{4}=45^{\circ}$, where the model is approaching the omaginary cup of coffee to grab 

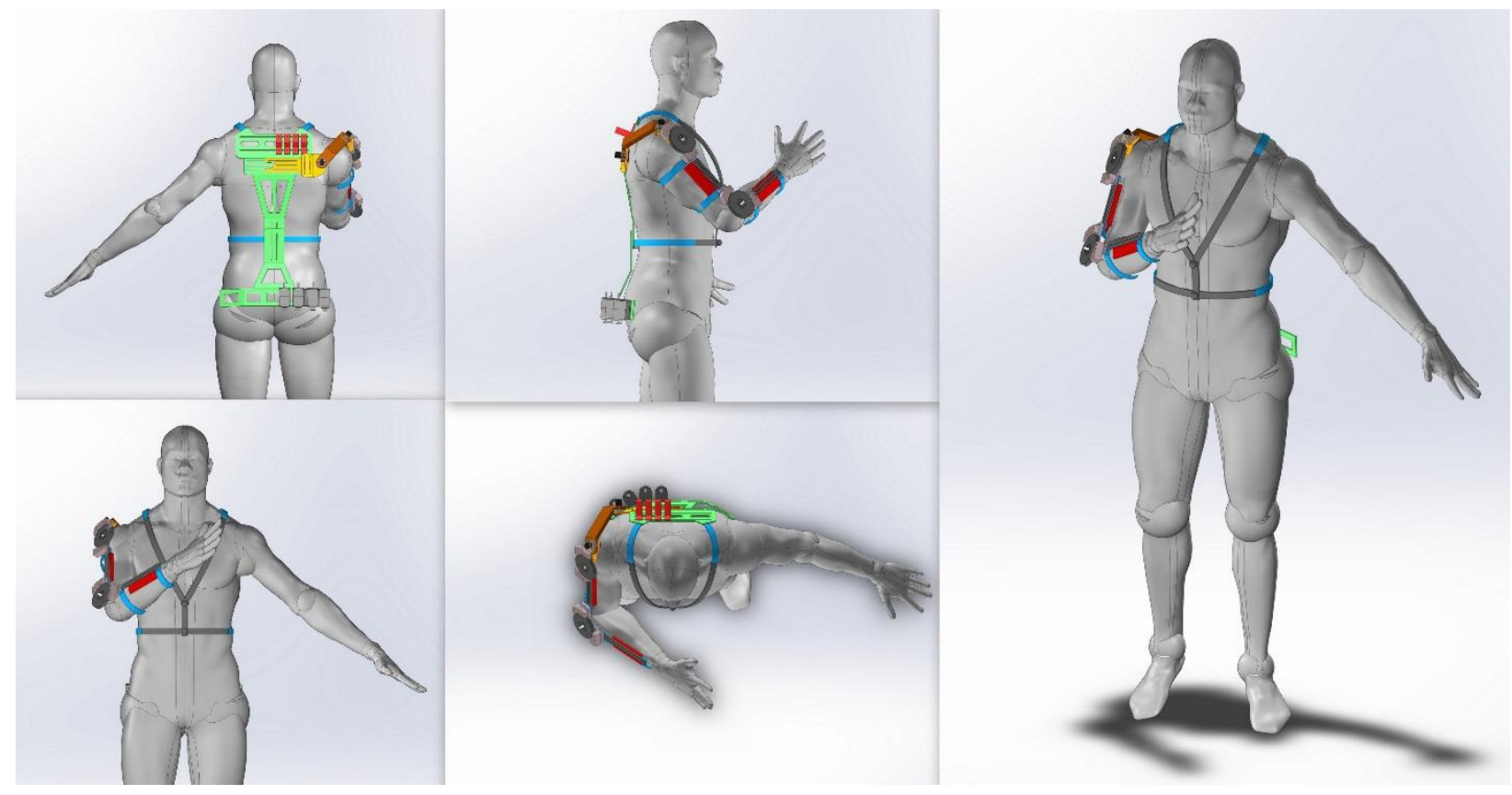

Figure 3. 28. The position of limbs when $\psi_{1}=10^{\circ}, \psi_{2}=60^{\circ}, \psi_{3}=0^{\circ}$, and $\psi_{4}=90^{\circ}$, where the model is approaching mouth to drink coffee
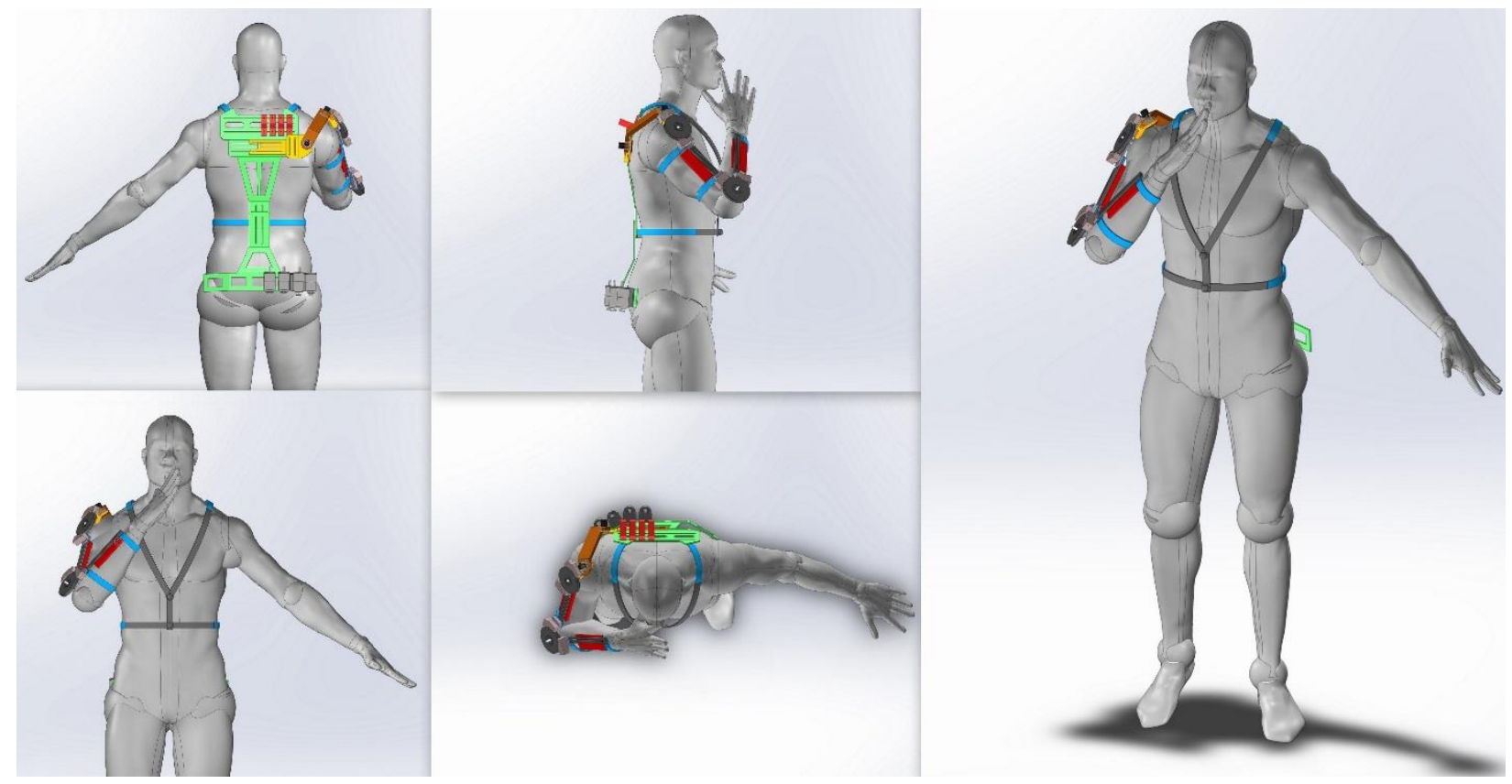

Figure 3. 29. The position of limbs when $\psi_{1}=28^{\circ}, \psi_{2}=55^{\circ}, \psi_{3}=20^{\circ}$, and $\psi_{4}=130^{\circ}$, where the model is drinking coffee from the imaginary cup of coffee 


\section{Chapter 4: Twisted String Actuation}

\section{Design}

\subsection{Introduction}

In this chapter a novel actuation method called twisted actuation was introduced. This method was selected mainly because it was light and can provide high power in a compact area. Thus, in the first part of the chapter the concept of the twisted string actuation method was presented. To investigate the performance of the twisted actuation, various strings were tested. For this purpose, a test bed was designed and fabricated to demonstrate the applicability of the twisted string actuation and its performance. Various lines were examined in order to find the best line with highest rigidity, best linearity, and fastest response, and pros and cons of each one were discussed. Then, based on the requirements, limitations of the mechanical design, and the results of the experiments, one of them was chosen to perform an ADL. Ultimately results were analyzed and a conclusion was made. 


\subsection{Twisted String Actuation Modeling [203]}

The basic concept of the twisting actuation method is simple. With one end fixed on an actuator shaft (electric motor) and the other end fixed on the load, two or more parallel strands can be twisted by the rotational movement of actuator. As the twist starts, the string connected to the motor shaft starts to reduce the distance between both ends and generates linear motion.

A kinetostatic model for such a transmission mechanism has been developed by Würtz et al [203]. They also proposed and used a simplified model, which assumed that the stiffness of strands was infinite. Notice that this assumption is correct only if the exerted force on the strands is small relative to the elastic modulus of the string. It should be also noted that the following model was entirely derived and proposed by Würtz and it will be only used to explain the behavior of the actuation. Omitted factors such as flexibility of the strings in the lateral direction and variation of the effective radius are the limitations of the current model. Thus, each actuation were tested in order to be used for control and the experimentally derived model was used instead of the theoretical model

Two strand configurations exist in the twisted string actuation, as shown in Figure 4. 1 illustrate that using a neutral strand increases the effective radius about which the strands are wrapped. The distance $r$ between the effective strands and center of rotation becomes $r=r_{s}+r_{c}$, where $r_{s}$ is the radius of the central neutral strand, and $r_{c}$ is the radius of the effective strands. The value of $r$ depends on the geometry of the actuation as shown below. 


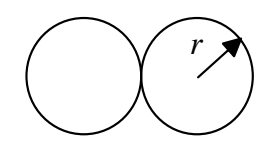

(a)

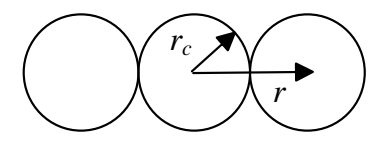

(b)

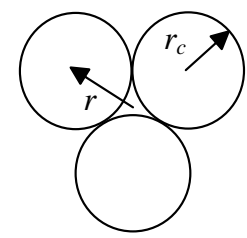

(c)

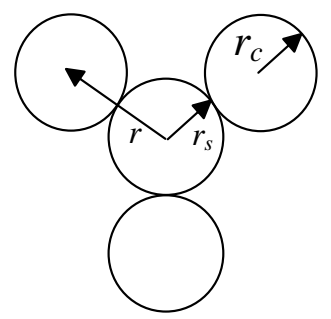

(d)

Figure 4. 1. Twisted string configuration of a two-string actuation ((a) and (b)) and three-string actuation ((c) and (d)) with and without a neutral strand [203]

The kinematic relationship between the motor rotation $\theta$ and the length of the transmission system $p$ is derived according to the Pythagoras' theorem, which is shown in Figure 4. 2. The actuation length $p$ is a function of the number of rotations of the actuator:

$$
p=\sqrt{L^{2}-\theta^{2} r^{2}}
$$

where $L$ is the total length of the string. Starting from completely untwisted strings the change in the actuation length $\Delta p$ will be

$$
\Delta p=L-\sqrt{L^{2}-\theta^{2} r^{2}},
$$

To maintain the static equilibrium at different positions, sufficient torque $\tau_{m}$ must be generated by the actuator. In other words, to stay at equilibrium, the static compound force $\left(n F_{i}\right)$ generated by $F_{z}$ as the tangential force (load), and $F_{\tau}$ as the axial force corresponding to $\tau_{m}$ must be aligned with the physical direction of the unwrapped helix. To provide dynamic actuation, the provided torque should be more or less than the required static torque. The amount of actuator force depends directly on the amount of load and helix slope of the strand, which is

$$
\begin{gathered}
F_{i}=F_{z} / n \cos (\alpha), \\
F_{\tau}=n F_{i} \sin (\alpha), \\
\tau_{m}=r F_{\tau},
\end{gathered}
$$




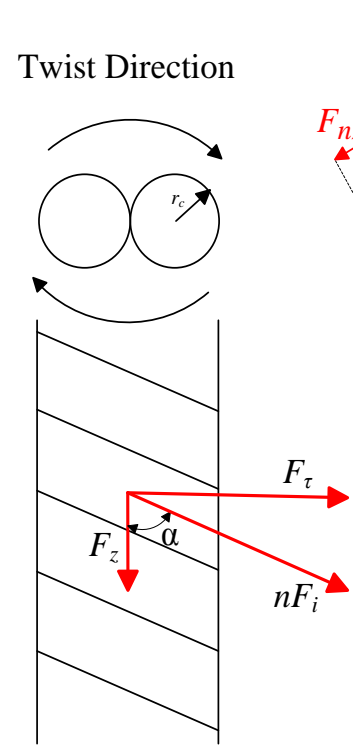

(a)

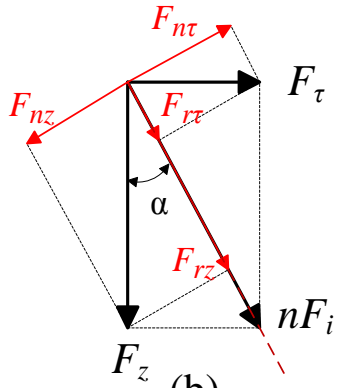

(b)

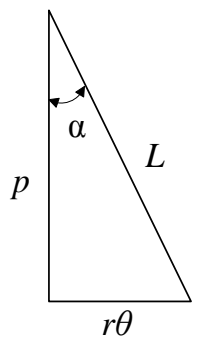

(c)

Figure 4. 2. (a) Schematic representation of twisted string and forces (b) Equilibrium of acting forces (c) unwrapped helix [203].

where $n$ and $\alpha$ are the number of strands and helix slope respectively. Combining equation of (4. 3), the torque $\tau_{m}$ becomes

$$
\tau_{m}=r F_{z} \tan (\alpha)=F_{z} \theta r^{2} / p,
$$

In cases of high loads or accelerated motion, the assumption of infinite stiffness of the string is no longer valid and the elongation of the strands due to the axial force must be taken into account. In that case, the total length of a string $L$ is a function of the tension $F_{i}$, the strand stiffness $k$, and its unloaded length $L_{0}$. Thus, (4. 1) can then be modified to

$$
p=\sqrt{L_{0}^{2}\left(1+\frac{F_{i}}{L_{0} k}\right)^{2}-\theta^{2} r^{2}},
$$

Using (4. 5), the equation to determine the length of pre-twist actuation can be explained as 


$$
p_{\theta_{0}}=\sqrt{L_{0}^{2}\left(1+\frac{F_{i}}{L_{0} k}\right)^{2}-\theta_{0}^{2} r^{2}}
$$

where $\theta_{0}$ is the number of revolutions of the actuator associated with the pre-twist. Assuming that this is the initial length of the cable, $p(\theta)$ as a function of the revolution of the motor can be derived as follows:

$$
P\left(\theta, F_{i}\right)=\sqrt{L_{0}{ }^{2}\left(1+\frac{F_{i}}{L_{0} k}\right)^{2}-\theta^{2} r^{2}}-\sqrt{L_{0}^{2}\left(1+\frac{F_{i, \theta}}{L_{0} k}\right)^{2}-\theta_{0}^{2} r^{2}},
$$

where $P\left(\theta, F_{i}\right)$ is the amount of displacement from the pre-twist position. According to the geometric relationship, there exists a maximum angle $\alpha$, where all strands are wrapped tightly on each other. After this point strands begin entangling around themselves. With the maximum angle $\alpha$, the pitch parameter $q$ of the helix is minimized.

$$
q_{\min }=2 n r_{s},
$$

On the other hand, the pitch parameter of a helix as a function of motor rotation angle $\theta$ and string length $p$ will be:

$$
2 \pi p=q \theta
$$

Assuming there is no load $\left(F_{z}=0\right)$ applied to the transmission and combining (4.5) with the geometry of the strands, (4. 8) and (4.9), the maximum achievable $\theta$ angle $\left(\theta_{\max }\right)$ and minimum contraction $\left(p_{\text {min }}\right)$ are respectively

$$
\theta_{\max }=\frac{L_{0}}{\sqrt{r^{2}+\frac{r_{s}^{2} n^{2}}{\pi^{2}}}} \text { and } \quad p_{\min }=\frac{L_{0}}{\sqrt{1+\frac{r^{2} \pi^{2}}{r_{s}^{2} n^{2}}}}
$$

The concept of the twisted actuation was used to develop a model for the actual actuation system for the robots. Two forces were applied to strings. One was applied by the weight of the arm and the other one was the force corresponding to the torque generated by the actuator. 


\subsection{Experiments with String Configurations and Types}

In this section a test bed, which was designed to test various strings will be presented. Then the results of testing all string will be shown and pros and cons of each one will be discussed.

\subsubsection{Test Bed Design}

To validate the proposed model and its reliability in different positions, several tests must be done. A test platform was built to characterize the relationship between the revolutions of the motor and the amount of contraction or expansion of the twisted string using different string types and configurations.

\subsubsection{Mechanical Design}

Two $400 \mathrm{~mm}$ Richelieu drawer slides were used to ensure the linear movement. The DC motor was mounted on the upper side of the test bed and contraction and expansion was performed by twisting the strings. A holder with 6 holes on a circle and one hole at the center was mounted on the shaft of the motor, which allowed testing any configuration of strings up to 6 [204]. The same holder was fixed on a slider, which transformed the rotational motion to linear motion.

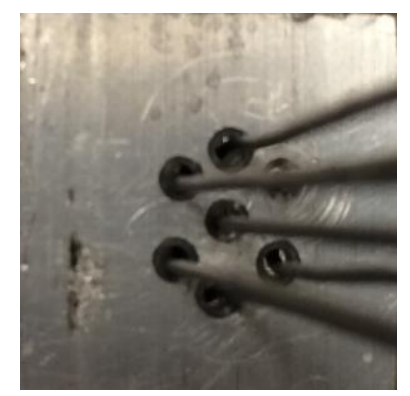

Figure 4. 3. String holder 


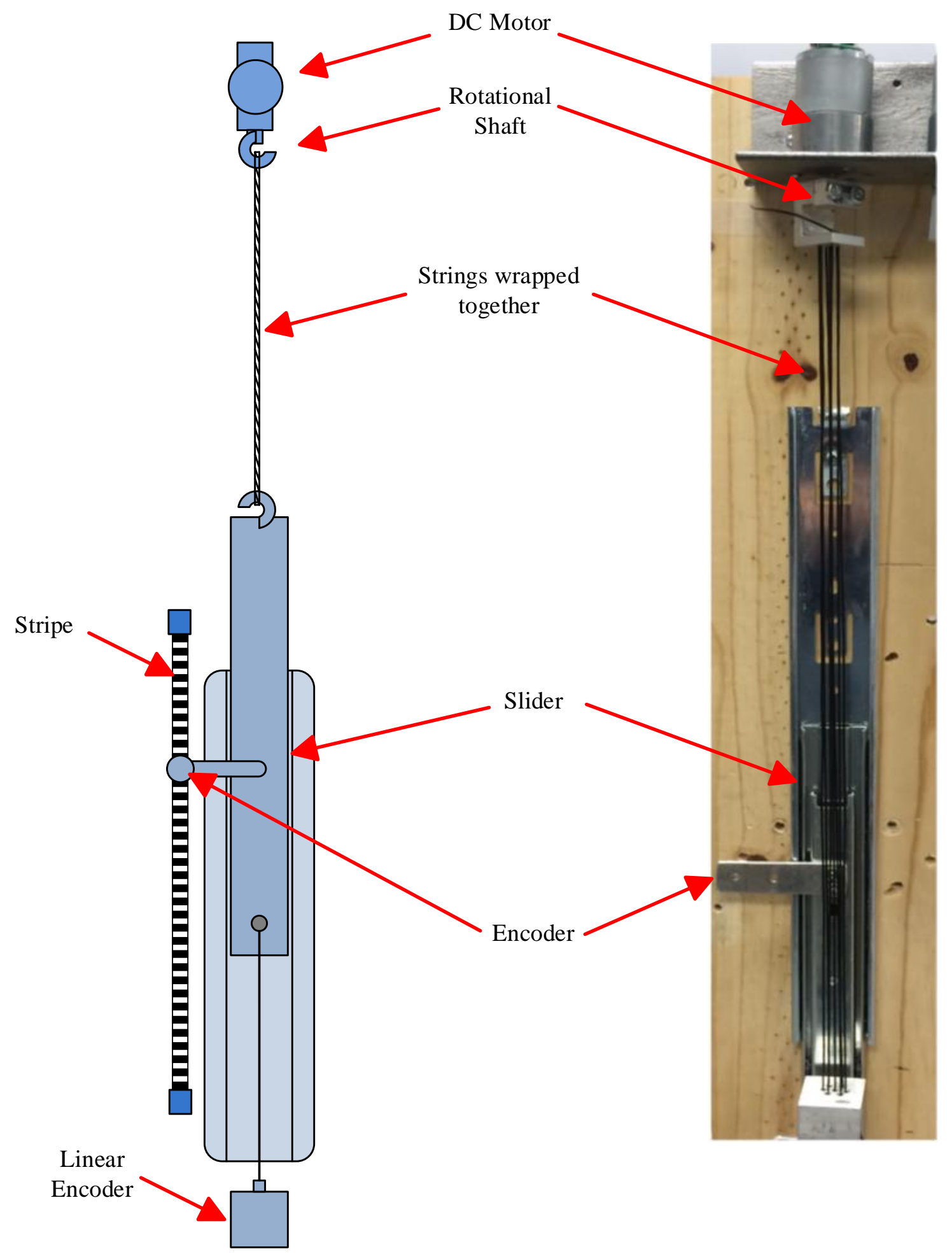

(a)

(b)

Figure 4. 4. (a) Schematic of test stand (b) actual test stand 
Ideally, the friction of the slides should be negligible in comparison with the load force $\left(F_{z}\right)$. To determine the friction of the slides, a simple model based on coulomb friction was used, which is presented schematically in Figure 4. 5 .

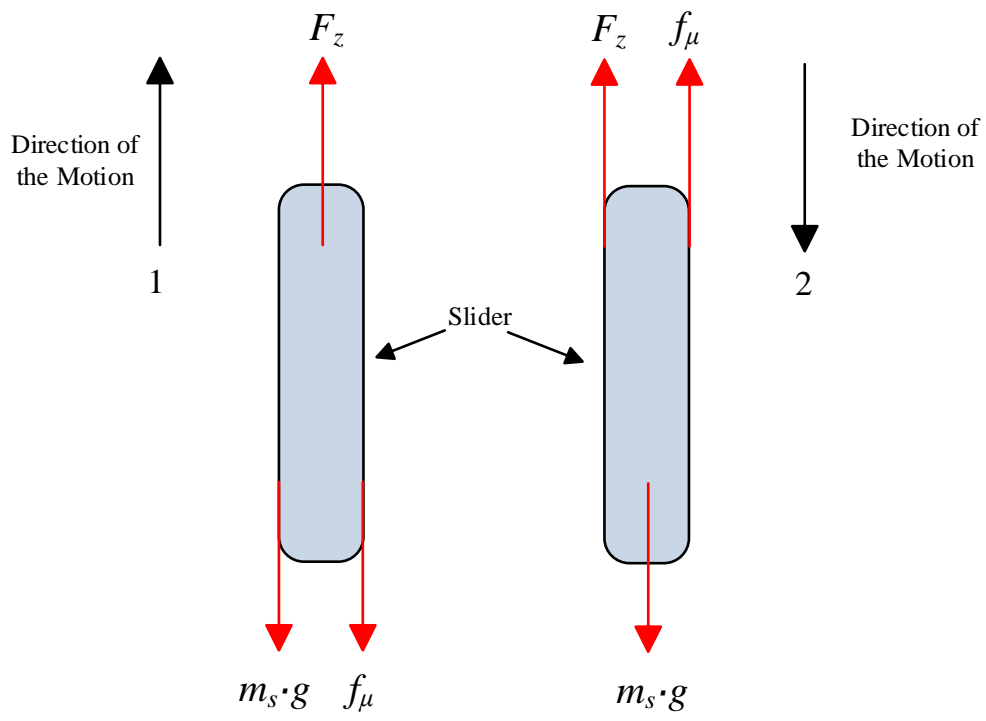

Figure 4. 5. Model of the slider with constant velocity

1) $F_{z}-\left(m_{s} g+f_{\mu}\right)=0 \longrightarrow f_{\mu}=F_{z}-m_{s} g$,

2) $F_{z}+f_{\mu}-m_{s} g=0 \longrightarrow f_{\mu}=m_{s} g-F_{z}$,

To make the motion stable, a 500g mass was added to the sliders. Each slider consists of two "stages" or sets of extension tracks that engage sequentially. The weights of the slider and load together for the first and second stages were $8.4 \mathrm{~N}$ and $10.2 \mathrm{~N}$ respectively in both the contraction and extension directions of motion. The weight of the second stage was bigger than the first stage since the weight of the second stage added up to the first stage when second stages started and it was inevitable. Having different stages with different weights, however, could show the response of the actuation in presence of a step load input. To estimate the friction of the sliders, there $\mathrm{w}$ moved upward and downward with constant velocity. The applied force $\left(F_{z}\right)$ was measured by an 
analog force gauge with $10 \mathrm{~Hz}$ sampling frequency moving up and down with sliders. The test was performed 10 times and results showed the same pattern for all tests.

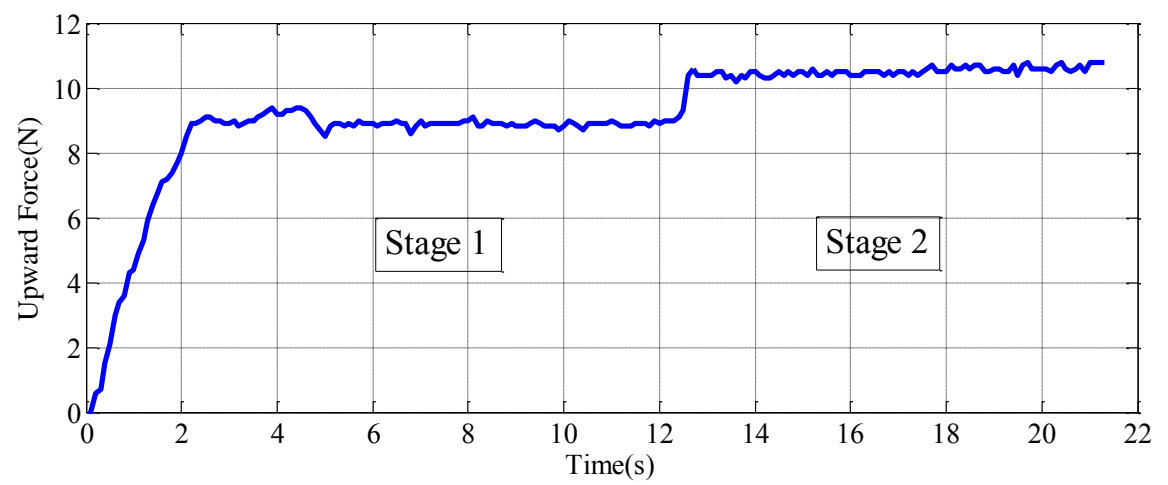

(a)

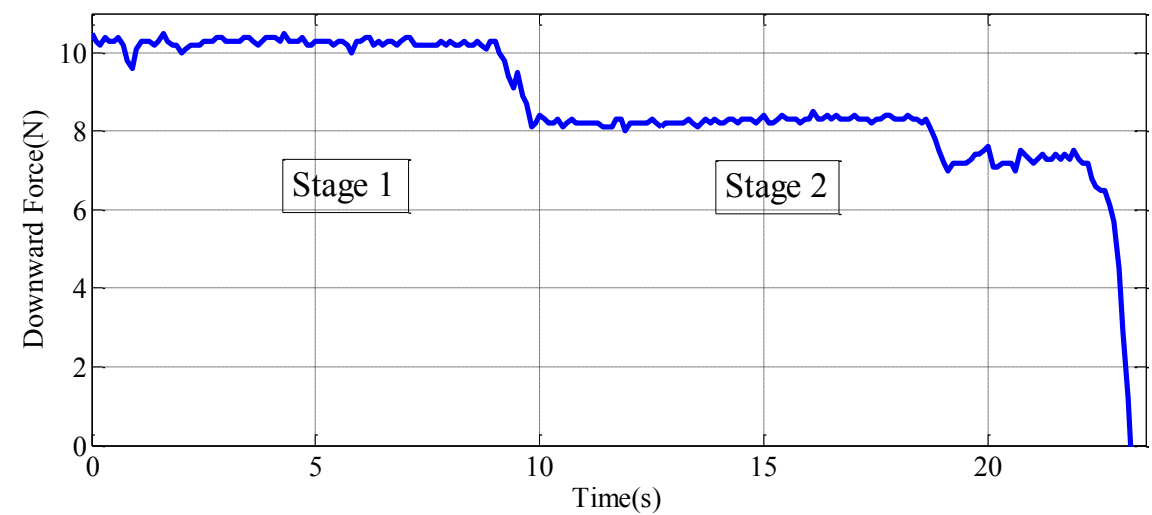

(b)

Figure 4. 6. Axial force for upward and downward motion

The applied forces for the upward and downward motion, including an estimate of the frictional forces were computed using (4.11).

Table 4. 1. Friction of the slider in stage one and two and in two directions

Friction $(\mathrm{N})$

\begin{tabular}{lcc}
\cline { 2 - 3 } & Stage 1 & Stage 2 \\
\cline { 2 - 3 } Upward motion & 0.52 & 0.30 \\
\hline Downward motion & 0.14 & -0.06
\end{tabular}


As shown in Table 4. 1, there was a great deal of inconsistency in the data, which may be due to human error since this test was done by a human hand. Thus it is probable that small errors in moving the mass up and down made difficulty of making accurate measurements of small forces. In any case, the highest measured value of the friction $(0.52 \mathrm{~N})$ was only $5 \%$ of the minimum load $(8.2 \mathrm{~N})$, and was much smaller than the anticipated loads for the actual robotic mechanism. Friction effects was therefore neglected in the next tests.

\subsubsection{Electronics and Sensor Systems}

As for the electronics a Mabuchi DC Motor with a 400 line rotary encoder was used for the monofilament fishing line and a high-torque DC motor with 19:1 gear ratio and 64 counts per revolution (CPR) encoder for the tactical and heavy duty cables. The primary reason of changing the DC motor for the tactical and heavy duty cables was the need to provide high torque to observe the behavior of the strings in high twist mode.

The movement of the slider was measured by a linear encoder with a resolution of $2 \pm 1 \mathrm{~mm}$ for the monofilament fishing line. A more accurate linear encoder with $0.4 \pm 0.2 \mathrm{~mm}$ resolution was used for the tactical and heavy duty cables. The major reason of using a more accurate sensor for the last two cables was the need to examine them under different loads and ultimate decision to use the last two cables for the control test. 


\subsubsection{Twisted String Actuation Tests and Results}

In this section the test results of various strings are presented. Discussing different configurations of strings, hysteresis, load sensitivity, linearity, and oscillation of actuation are the objectives of this section.

\subsubsection{Test Procedure}

Three types of strings were tested in this work: a monofilament fishing line, a utility cable, and a tactical cable. In these tests the behavior of the actuation within the low hysteresis area was investigated. Since the actuations were twisted to the maximum limit and in some cases beyond, the low hysteresis area was determined for each configuration by testing firstly. Then the actuation was twisted to that limit and its behavior was recorded. The response of the actuation beyond this limit demonstrates hysteresis, and the higher the number of revolutions becomes, the bigger the hysteresis will be.

The monofilament fishing line was the first test, which was tested with two different lengths: $50 \mathrm{~cm}$ and $100 \mathrm{~cm}$. The results were obtained by performing each test for 50 cycles and selecting one sample after each consecutive 10 samples. The experiment was done under $500 \mathrm{~g}$ load and for one string configuration (section (a) of Figure 4. 7). There was no rest between each contraction and expansion. In other words, expansion started as the actuation reached the maximum contraction.

The utility cable and tactical cables were tested for 8 different configurations and under 4 different loads: $500 \mathrm{~g}, 1500 \mathrm{~g}, 2500 \mathrm{~g}, 3500 \mathrm{~g}$, which were close to the working loads for the elbow motion. The maximum load was limited to $3.5 \mathrm{~kg}$ due to current limitations of the DC motor in 
testing. The tests were done 5 times for each configuration under each load in order to ensure the consistency of the response and to demonstrate the hysteresis in the response if it exists. Since the reasonable length of the actuation at rest to be mounted on a wearable robot was within $50 \mathrm{~cm}$ of the maximum length of the cable, the behavior of the line was not tested for $100 \mathrm{~cm}$ anymore. The 8 different configurations are listed below.

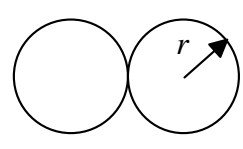

(a)

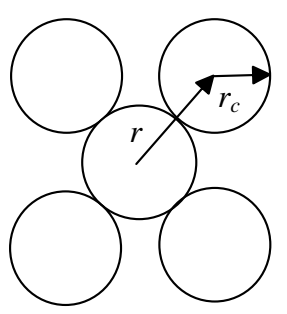

(e)

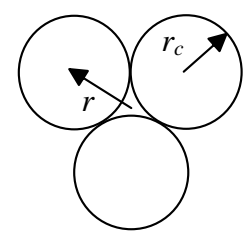

(b)

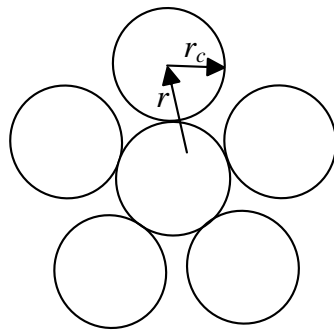

(f)

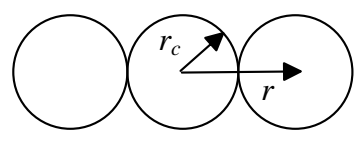

(c)

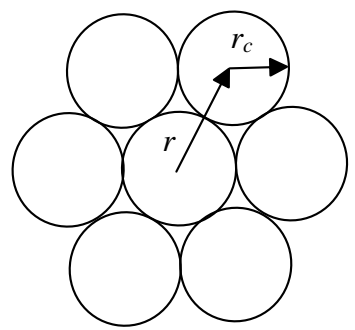

$(\mathrm{g})$

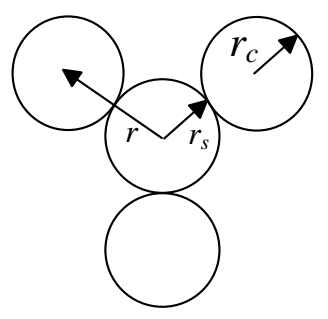

(d)

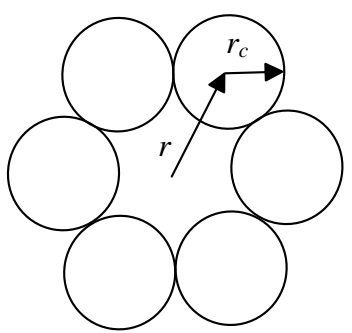

(h)

Figure 4. 7. Different configurations of the strings used in the experiment

\subsubsection{Monofilament Fishing Line}

In the first test, two conventional monofilament fishing lines (TEBCO Outcast) were selected with a maximum working load of $20 \mathrm{lb}$. each. The diameter of each strand of the fishing line was 0.5 according to its catalog, which was validated by a digital caliper. The shape of the filament was confirmed to be perfectly round by measuring the diameter along the length before testing. However, after several testing not only the diameter of the string, but also the overall shape of the string may be affected based on how much it was twisted or if it reached the plasticity range or 
not. Results of measuring the diameters showed that the actual diameter is $0.5 \pm 0.05 \mathrm{~mm}$ (error of the digital caliper).

Figure 4.8 shows that the experimental results deviated significantly from those predicted by the model. The difference can be due to the elastic behavior caused by the additional force created by the attached weight or perhaps by another factor, such as ignoring the friction between the strings.

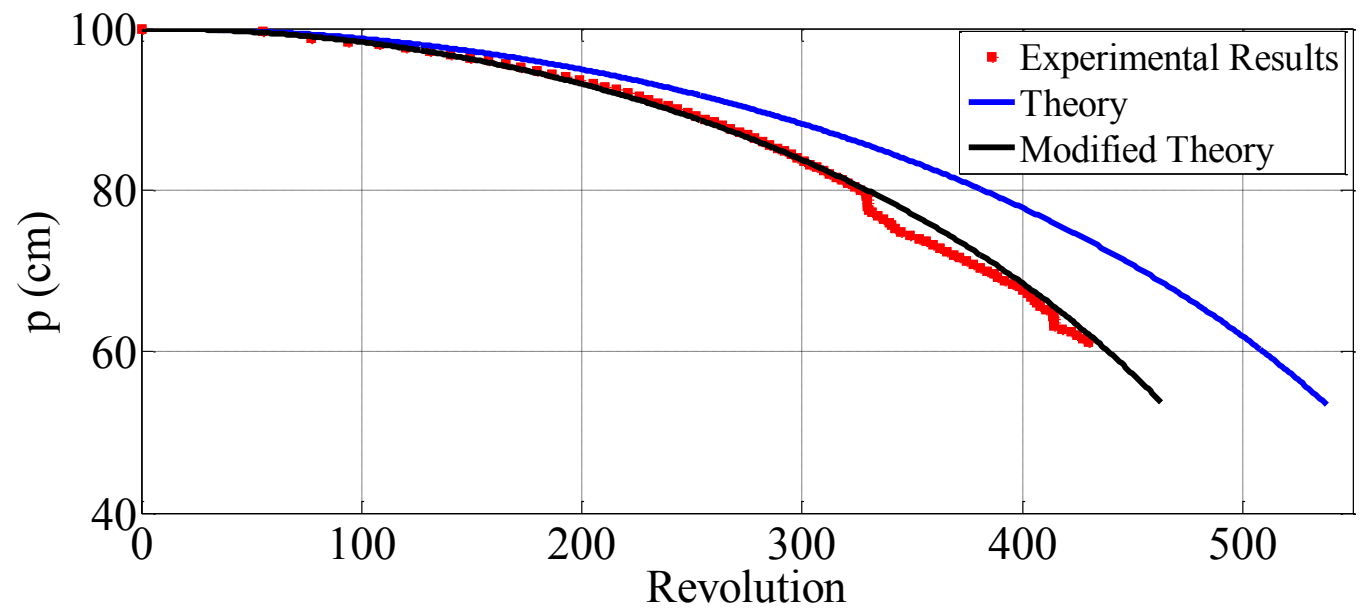

Figure 4. 8. Comparison of the theoretical transmission length and experimental measurement for $100 \mathrm{~cm}$ length

Other than the discrepancy, the discontinuity at 320 revolutions was due to a mechanical artifact of the test apparatus. And the jump at 410 revolutions was due to the permanent distortion caused by the over-twisted phenomenon. To adapt the data from experiment to the model, an effective diameter for the strands was suggested, which was greater than the diameter from measurement. The suggested value was $0.55 \mathrm{~mm}$. The behavior of the model considering the effective diameter is also shown in Figure 4. 8 (black line). According to observations and comparing results to the model using the effective diameter, kinking occurred sooner than what the model predicted. Therefore, the model provided an approximate revolution in which actuation enters the nonlinear area; however, to acquire the exact kinking point, the line should be tested experimentally. The model suggested that kinking should occur at 460 revolutions. However, 
kinking happens at 410 revolutions. Thus, it is suggested that the maximum number of twists for the adopted fishing line for the two-string configuration is no more than 400 rev.

To ensure the behavior consistency of the string mechanism, the strands must be tested under repetitive motion/force. Figure 4.9 demonstrates the repetitive motions of both contraction and expansion for different string lengths $(50 \mathrm{~cm}$ and $100 \mathrm{~cm})$.

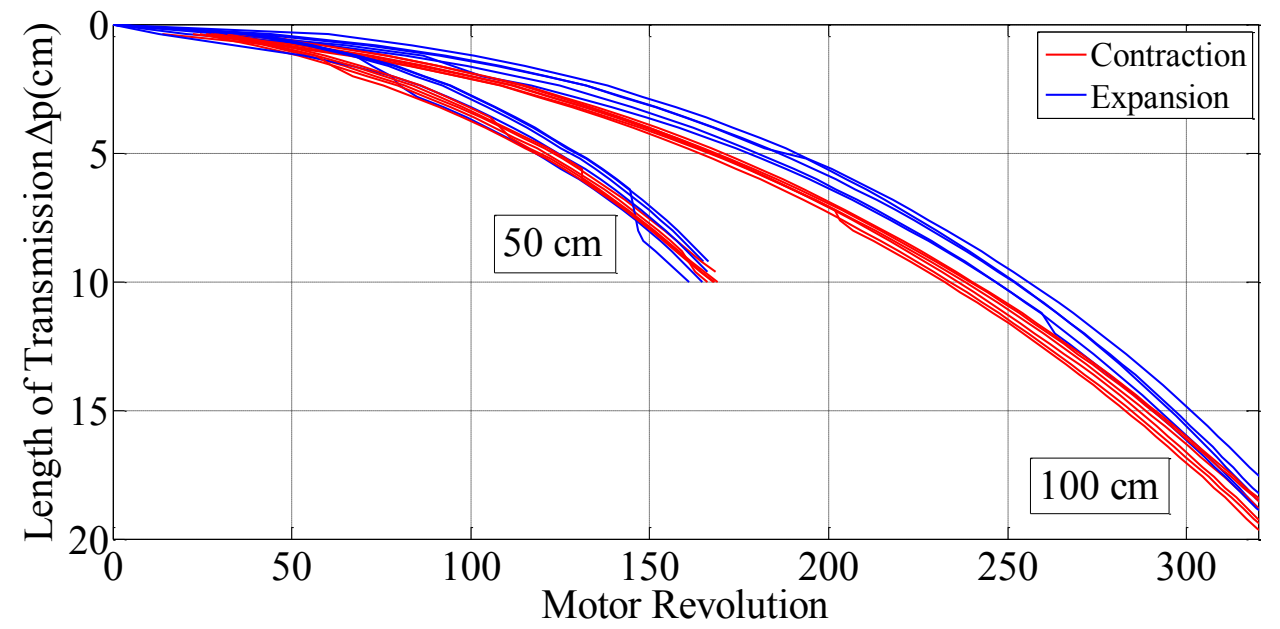

Figure 4. 9. Repetitive tests of the twisted actuation system with different transmission length

As shown in Figure 4. 9, actuation responses of contraction or expansion separately do not match. In other words the trajectories of the actuation for 5 different contraction or expansions are different. Moreover the trajectory of the same set of data for contraction and expansion did not match, which means that there was hysteresis in the system.

From the control point of view, hysteresis should be measured since high hysteresis generates error and increases control effort. To investigate the hysteresis of this phenomenon two sets of data corresponding to $50 \mathrm{~cm}$ and $100 \mathrm{~cm}$ were selected, which are shown in Figure 4. 10. The test sets were chosen from the five test set and results are shown in Figure 4. 10. The shown data were the worst case responses of actuation under the same load and testing conditions. 


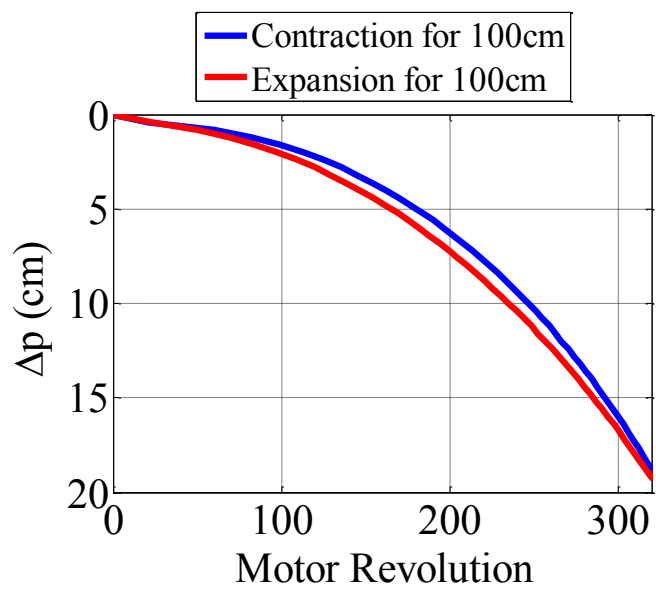

(a)

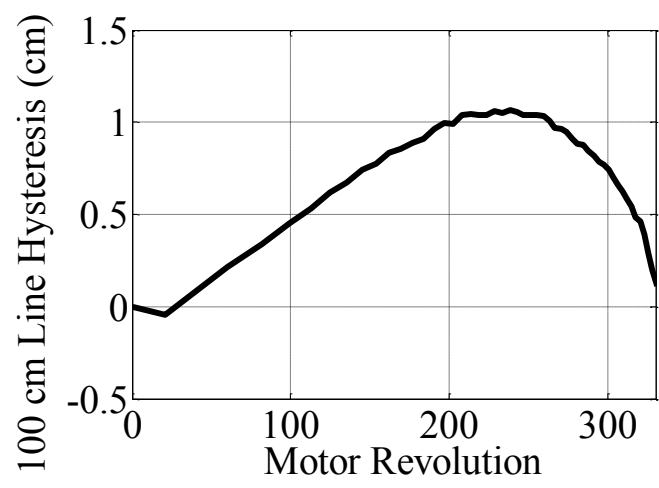

(c)

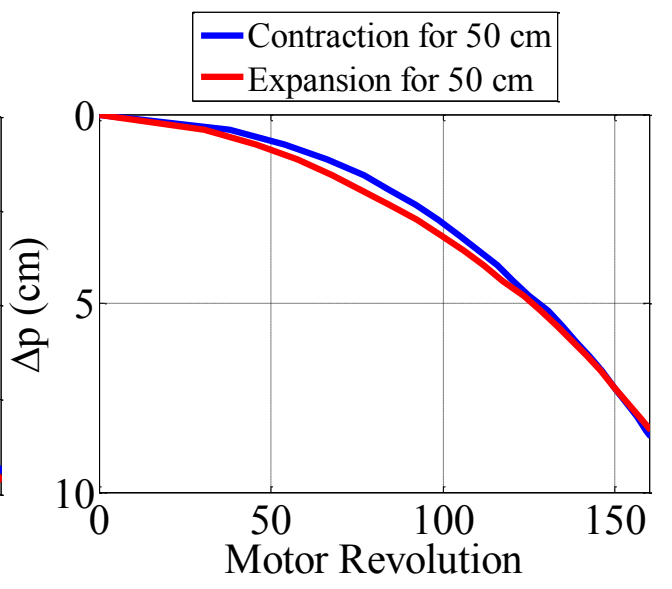

(b)

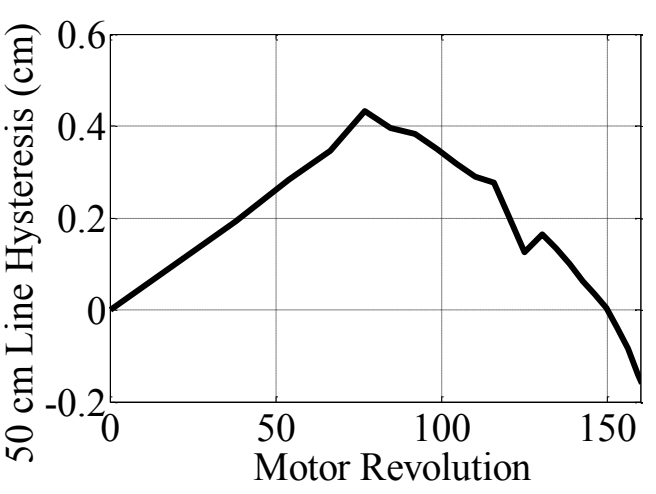

(d)

Figure 4. 10. $\Delta \mathrm{p}$ of contraction and expansion for (a) $100 \mathrm{~cm}$, and (b) $50 \mathrm{~cm}$ Hysteresis of contraction relative to expansion for (c) $100 \mathrm{~cm}$, and (c) $50 \mathrm{~cm}$

As shown in Figure 4. 10, hysteresis increase as the actuator revolution increases and after reaching a peak at half of the motor revolution it decreases. The same trend was observed for both contraction and expansion. The maximum hysteresis for $100 \mathrm{~cm}$ long string was $1 \mathrm{~cm}$ and for 50 $\mathrm{cm}$ long string $0.4 \mathrm{~cm}$

\section{Discussion}

In this experiment a fishing line with two actuation lengths was tested. Although the fishing line was made of plastic, the surface was not as slippery as expected, which could be the source of 
hysteresis. In addition, the friction of slides and measurement errors must be taken into consideration. Moreover, the response of the system was based on the assumption that no axial load is applied to the string to simplify the equation. With the assumption that there was an axial force applied to actuation, more deviation would be generated.

Since the model did not match the experimental data an effective diameter of string was obtained to fit the model to experimental data. The effective diameter was larger than the actual diameter of the line by about $10 \%$. If the strands were twisted more than the maximum allowed twist angle, the line began kinking, which was the major source of unexpected nonlinear behavior and abrupt jumps. It should be noted that the kinking distorts the strands permanently and makes them unpredictable for control. It was also observed that the kinking occurred sooner than the theory - considering the effective diameter - predicts.

\subsubsection{Utility Line}

Due to high elasticity of the fishing line as well as slow contraction, it was decided to use a more rigid string with higher working load limit in order to perform tasks with bigger loads. Moreover, the string should be thicker in order to increase the contraction response of the actuation. For the second test, a utility line with $0.9 \mathrm{~mm}$ thickness and $13.6 \mathrm{~kg}$ maximum working load was selected.

\section{Two-string Actuation}

The responses of the two-string actuation with and without the neutral string are shown below. The maximum number of motor revolutions within the low-hysteresis area for the actuation with and without neutral string were 47 and 60 revolutions (shown with arrows) subsequently. 
2-string (2S.) actuation with a neutral string (1N.) and without $(0 \mathrm{~N}$.)

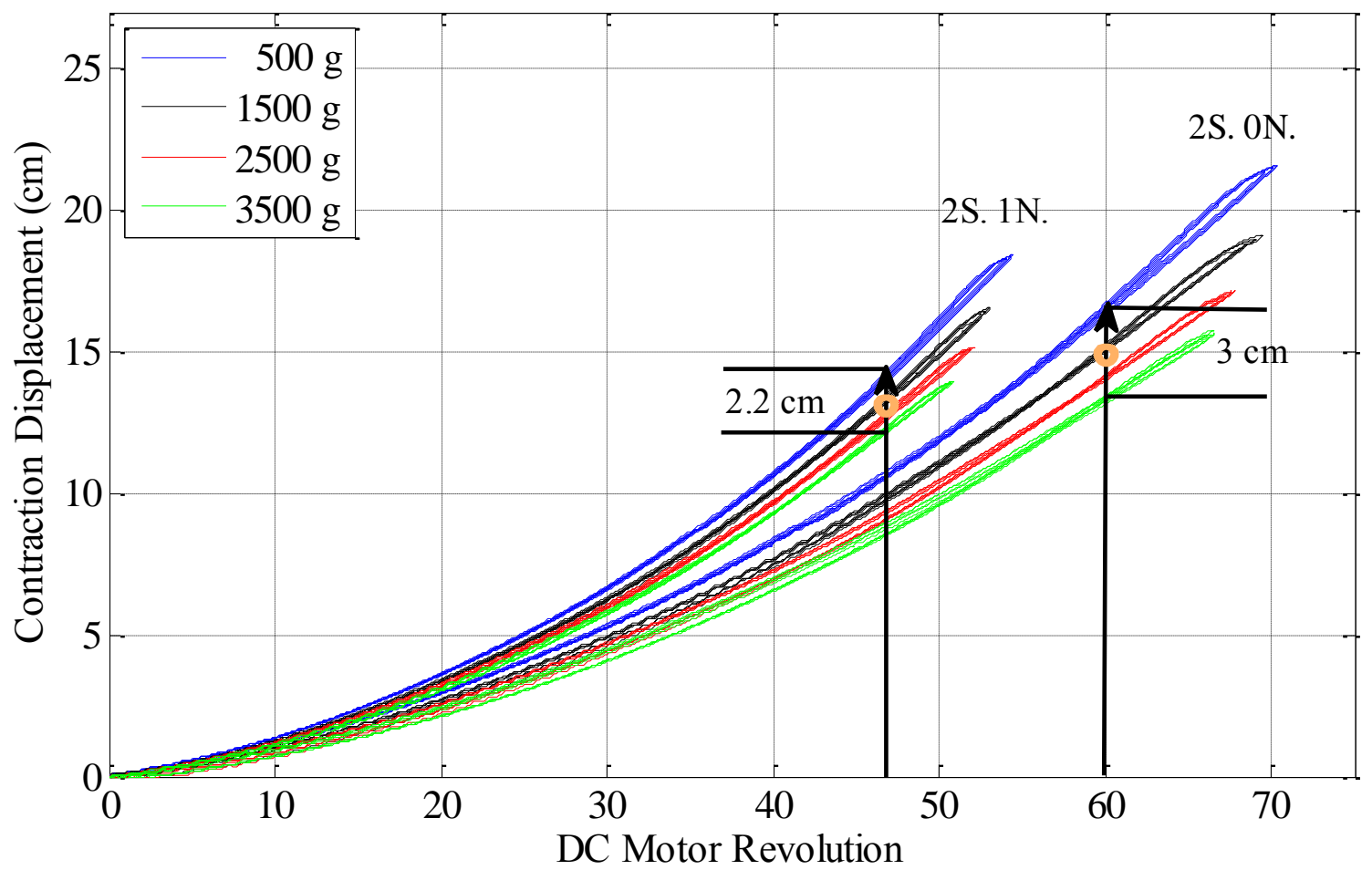

Figure 4. 11. Two-string actuation with and without a neutral string

As shown in Figure 4. 11, adding a neutral string reduced the maximum average number of revolutions without going to the high-hysteresis area from almost 60 revolutions to 47 revolutions, which demonstrated almost $20 \%$ improvement in the actuation response. This reduction was because of the $100 \%$ increase in the effective radius $(r)$ shown in Figure 4. 7. Moreover, the maximum contraction of the average of four tests (shown with an orange dot) decreased from 15 $\mathrm{cm}$ for the actuation without a neutral string to $13.5 \mathrm{~cm}$ with a neutral string, which demonstrated a $10 \%$ reduction in full contraction. Using a neutral string improved precision of actuation too. The actuation without a neutral string demonstrated $3 \mathrm{~cm}$ variation under various loads at maximum contraction, while the same configuration with a neutral string demonstrated $2.2 \mathrm{~cm}$ variation $(25 \%$ reduction). 
Oscillatory motion of the actuation at low revolutions was one of the major phenomena observed in this experiment. Oscillatory motion of a system amplified once the working frequency was close to the natural frequency of the system [205]. Since the test stand was stabilized through bolts and screws and the DC motor was working with high speed (which is close to the working area), the only parameters playing a significant role in the amplification of the oscillatory motion were the spring coefficient of the strings and various loads. Investigating the origin of the oscillatory motion as well as determining the spring constant of cables were beyond the scope of this research. However, this oscillation should be considered once the proper actuation needs to be selected.

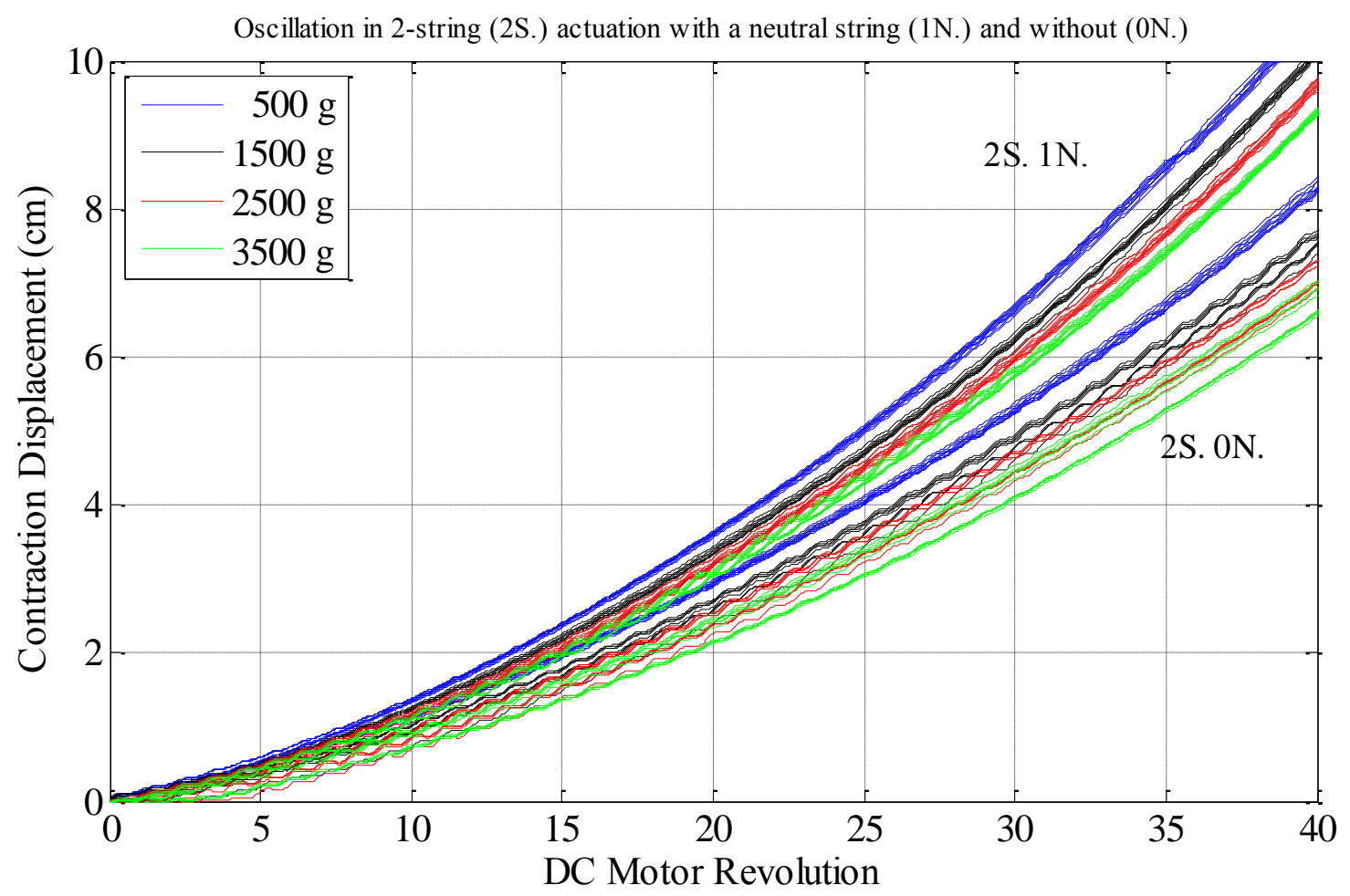

Figure 4. 12. Oscillatory motion of two-string actuation with and without a neutral string

As shown in Figure 4. 12, both cables exhibited some oscillatory response at low revolutions where strings were being twisted without much contraction. This phase started from the resting 
station, where the strings were parallel and untwisted and eventually the oscillations were damped at higher revolutions. The oscillatory motions was observed for both contraction and release and they were damped much faster for the configuration with a neutral string than without a neutral string for all loads.

The oscillations of the two-string actuation without the neutral string for all loads were damped after the first 50 revolutions of contraction/release, which was the first $10-13 \mathrm{~cm}$ of the motion. This phase occupied almost the first $65 \%$ of contraction/release. However, the oscillation of the same configuration with a neutral string exhibited more robust response. All oscillations for a two-string actuation with a neutral string were damped after the first 30 revolutions of the motion, which was the first 5-6 cm of contraction/release. This phase occupied only $35 \%$ of the nonhysteresis range of contraction/release.

\section{Three-string Actuation}

The responses of the three-string actuation with and without the neutral string are shown in Figure 4. 13. The maximum motor revolutions within the low-hysteresis area for the actuation with and without neutral string were 38 and 55 revolutions (shown with arrows) subsequently.

Similar to the previous actuation configuration, adding a neutral string reduced the maximum average number of revolutions (without going to the high-hysteresis area) from almost 55 revolutions to 38 revolutions (30\% increase in response speed), which demonstrated more improvement in comparison with the same one for two-active-string configuration. This reduction was because of $73 \%$ increase in the effective radius $(r)$. Moreover, the maximum contraction of the average of four tests (shown with an orange dot) was decreased from $15 \mathrm{~cm}$ for the actuation without a neutral string to $11 \mathrm{~cm}$ with a neutral string ( $25 \%$ reduction in full contraction), which was bigger than the same range for two active strings. The actuation without a neutral string 
demonstrated $1.5 \mathrm{~cm}$ variation under various loads at maximum contraction, while the same configuration with a neutral string demonstrated $1.3 \mathrm{~cm}$ variation (13\% reduction).

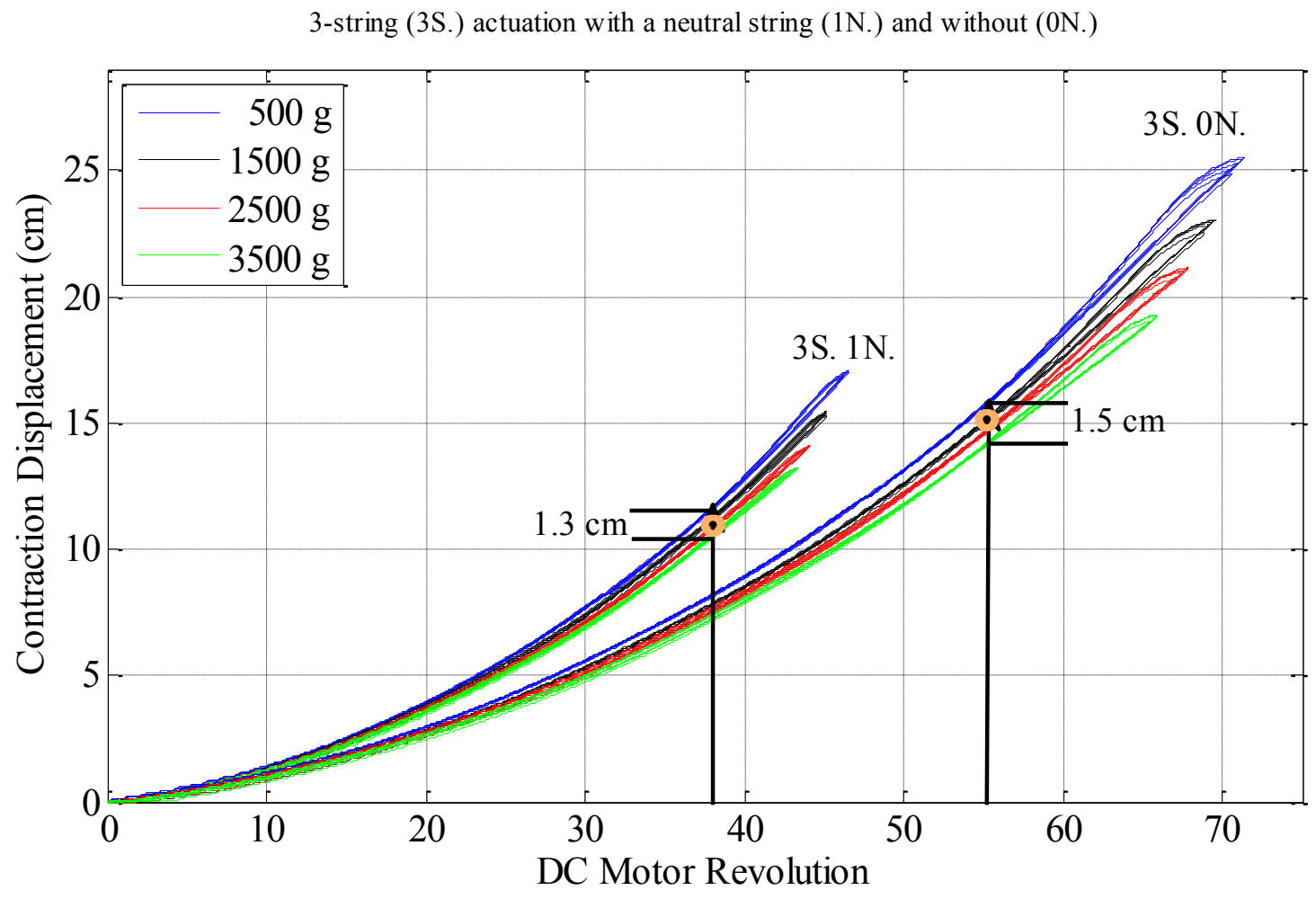

Figure 4. 13. Three-string actuation with a without a neutral string

Similar to the previous configuration, both actuations exhibited some oscillatory response in contraction and release at low revolutions, which were damped much faster for the configuration with a neutral string that without a neutral string. The oscillations of the three-string actuation without the neutral string for all loads were damped after the first 45 revolutions of contraction/release, which was the first $10-11 \mathrm{~cm}$ of contraction. This phase occupied almost the first $70 \%$ of contraction/release. However, the oscillation of the same configuration with a neutral string were damped faster. All oscillations for a two-string actuation with a neutral string were damped after the first 25 revolutions of the motion, which was the first $5-5.5 \mathrm{~cm}$ of contraction/release. This phase occupies $50 \%$ of the non-hysteresis range of contraction/release. 


\section{Four, Five, and Six-string Actuation}

The responses of the actuation with four, five, and six effective strings and a neutral string are shown in Figure 4. 14. The maximum number of motor revolutions within the low-hysteresis area for the four, five, and six-string actuations (shown with arrows) were 35, 31, and 31.5 revolutions respectively, which showed little difference.

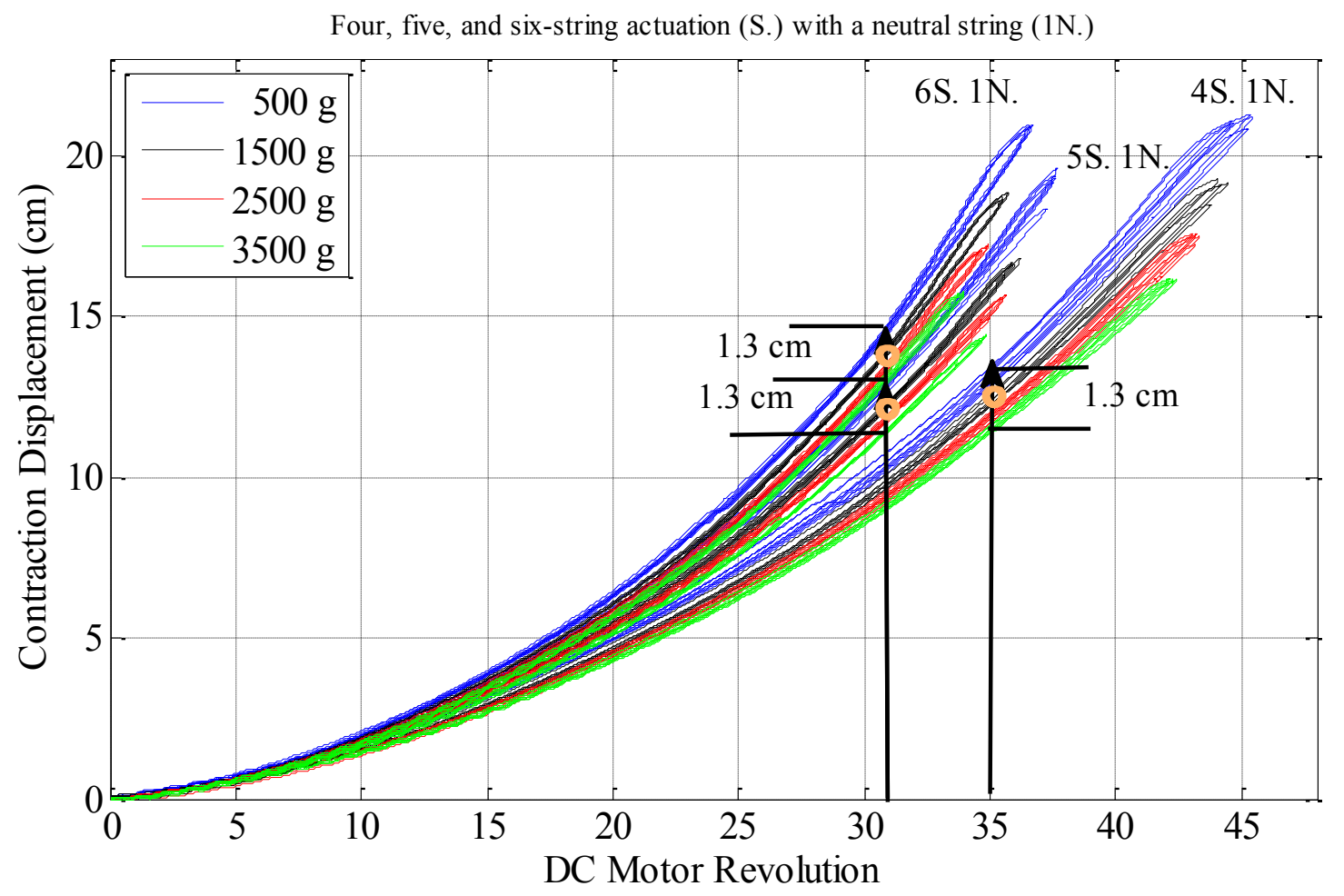

Figure 4. 14. Four, five, and six-string actuation with a neutral string

As expected, adding effective strings reduced the maximum average revolutions (without going to the high-hysteresis area). The maximum contraction of the average of four tests (shown with an orange dot) were $12.5,12.5$, and $14 \mathrm{~cm}$ for the four, five, and six-string configurations respectively. All actuations demonstrated almost $1.3 \mathrm{~cm}$ variation under various loads at maximum contraction. 
Similar to the previous configurations, all three of the new configurations exhibited some oscillatory response in contraction and release at low revolutions, which were damped in lower revolutions in comparison to the two and three-string configurations. The oscillations of the four, five, and six-string actuations were damped after the first 20, 17, and 16 revolutions of contraction/release subsequently (the first $4.5-5.0,3.7-4.1$, and $4-4.5 \mathrm{~cm}$ of contraction). This phase occupied almost the first $30 \%$ of the non-hysteresis range of contraction/release.

\section{Six-string Actuation}

The responses of the actuation with six effective strings and with and without a neutral string are shown in Figure 4. 15. The maximum number of motor revolutions (shown with arrows) within the low-hysteresis area for the actuation with and without the neutral string were 31.5 and 31 revolutions respectively, which showed little difference.

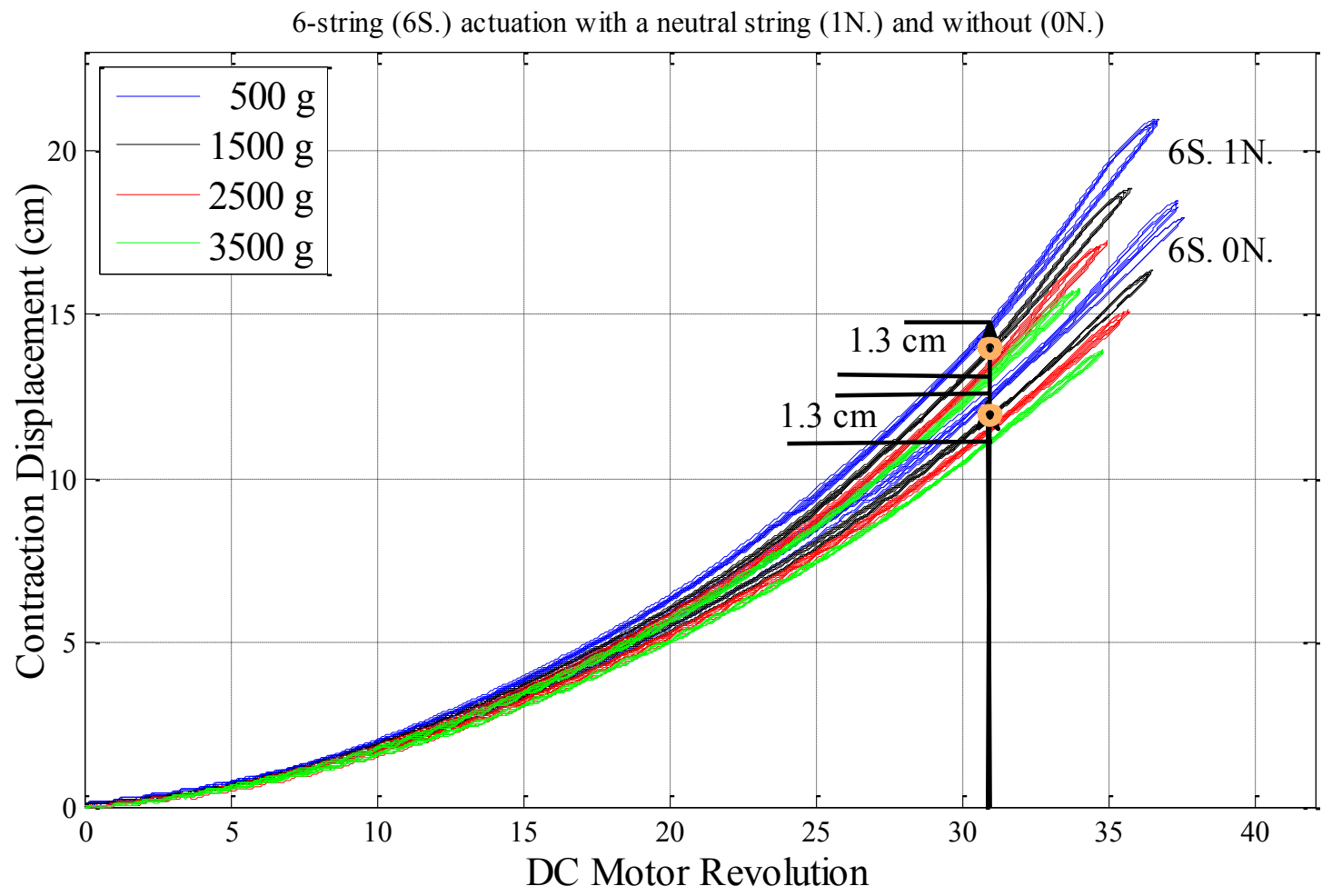

Figure 4. 15. Six-string actuation with a without a neutral string 
The maximum contraction consequently were $14 \mathrm{~cm}$ and $11.5 \mathrm{~cm}$ for the actuation with and without the neutral string. Unlike other configurations, adding a neutral string does not significantly change the behavior of the actuation. This was mainly because there were already multiple effective strings acting in the configuration. Adding a neutral string, however, could improve the reduction of the oscillatory motion. All oscillatory motions were damped after the first $8 \mathrm{~cm}$ of contraction for the actuation without a neutral string, while it was almost $4 \mathrm{~cm}$ for the same configuration with a neutral string (50\% reduction). Both actuations demonstrated almost $1.3 \mathrm{~cm}$ variation under various loads at maximum contraction.

\section{Discussion}

The summary of the characteristics of different actuations are listed below. As shown in Table 4. 2, adding a neutral string not only improved the oscillatory motion significantly but also decreased the maximum required number of revolutions of the DC motor, which increased the contraction speed. It also improved the non-oscillatory range almost $100 \%$ for all configurations.

The maximum non-oscillatory response was achieved using a six-string actuation with a neutral string with 9.5 to $10 \mathrm{~cm}$ contraction range. Moreover, additional effective strings made the motion less sensitive to load but reduced the maximum contraction, which was faster response with respect to the constant motor speed. In addition, increasing load increased the stability of the actuation and reduced the high-hysteresis range at high revolutions.

The responses of actuations under $3500 \mathrm{~g}$ load are shown in Figure 4.16 to demonstrate the trend of change in the behavior of the actuation system by adding more strings, Actuations with the same effective string configurations were colored similarly. 
Table 4. 2. Characteristics of different configurations of the utility cable strings

\begin{tabular}{lccccc} 
Actuation Type & $\begin{array}{c}\text { Maximum } \\
\text { DC Motor } \\
\text { Revolution }\end{array}$ & $\begin{array}{c}\text { Maximum } \\
\text { Contraction } \\
(\mathrm{cm})\end{array}$ & $\begin{array}{c}\text { Maximum } \\
\text { Variation due to } \\
\text { Load }(\mathrm{cm})\end{array}$ & $\begin{array}{c}\text { Oscillatory } \\
\text { Contraction } \\
\text { Range }(\mathrm{cm})\end{array}$ & $\begin{array}{c}\text { Range of Contraction } \\
\text { without Oscillation } \\
(\mathrm{cm})\end{array}$ \\
\hline 2 effective, 0 neutral & 60 & 15 & 3.0 & $10-13$ & $3.0-5.0$ \\
\hline 3 effective, 0 neutral & 55 & 15 & 1.5 & $10-11$ & $4.0-5.0$ \\
\hline 2 effective, 1 neutral & 47 & 13.5 & 2.2 & $5.0-6.0$ & $7.5-8.5$ \\
\hline 3 effective, 1 neutral & 38 & 11 & 1.3 & $5.0-5.5$ & $5.5-6.0$ \\
\hline 4 effective, 1 neutral & 35 & 12.5 & 1.3 & $4.5-5.0$ & $7.5-8.0$ \\
\hline 5 effective, 1 neutral & 31 & 12.5 & 1.3 & $3.7-4.1$ & $8.4-8.8$ \\
\hline 6 effective, 0 neutral & 31 & 11.5 & 1.3 & $7.5-8.0$ & $4.0-4.5$ \\
\hline 6 effective, 1 neutral & 31.5 & 14 & 1.3 & $4.0-4.5$ & $9.5-10$
\end{tabular}

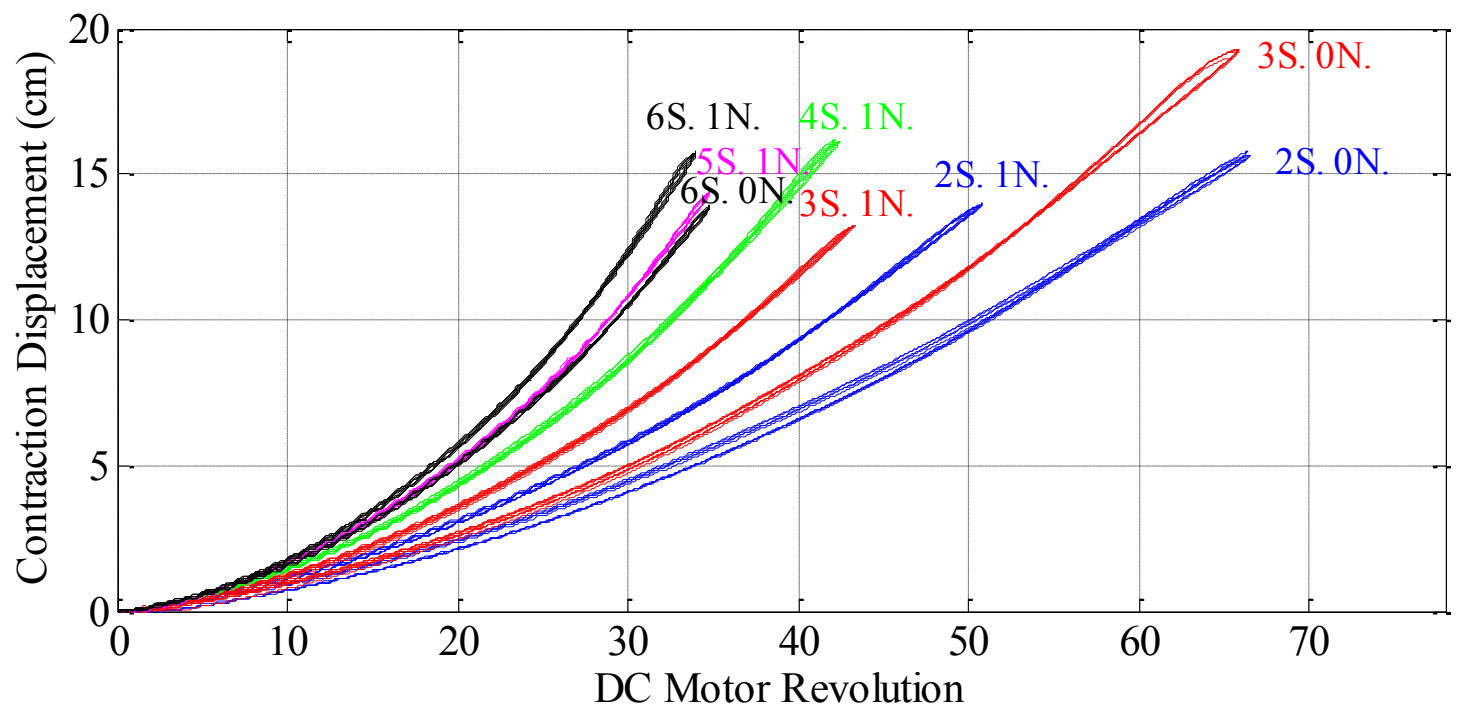

Figure 4. 16. The responses of the actuation system under $3500 \mathrm{~g}$ load with different string-configurations

One of the critical issue, which should be carefully considered in selecting a string was durability and wearing resistance. Thus, after 200 test cycles for different string configurations and mass loads, the two strings before testing and after use were compared. 


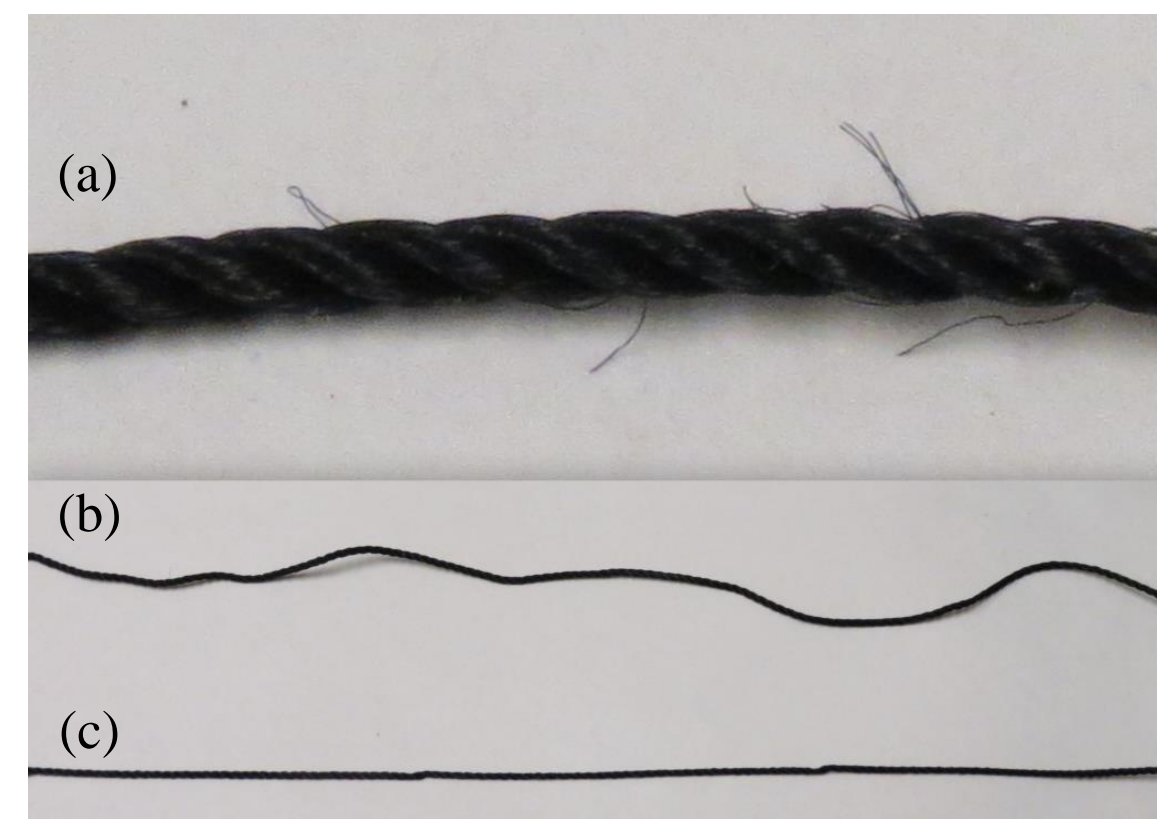

Figure 4. 17. (a) Worn out part of the sting (b) string after use (c) string before use

As shown in Figure 4. 17, the string was distorted after the work cycle. However, this distortion did not permanently damage resulting in change of behavior of the actuation. Moreover, the string was worn out, as shown in part (a) of Figure 4. 17. Since the string was not abrasive resistive, it should be constantly checked after a certain cycle period in order to avoid getting torn up.

\subsubsection{Tactical Cable}

Although utility line provided fast contraction, it was still sensitive to load. Thus a more rigid string with higher working load limit was selected in order to perform tasks with bigger loads. Moreover, the new line was thicker in order to increase the contraction speed of the actuation.

A tactical cable with 0.08 inch thickness and 450 lbs. (as stated in catalog) maximum working load was selected. This cord was made of an abrasion-resistant material (Technora), which had 
low stretch and high strength. Moreover, the PTFE (Teflon) coating increased the abrasion resistance protection [206].

\section{Two-string Actuation}

The responses of the two-string actuation with and without the neutral string are shown below. The maximum number of motor revolutions within the low-hysteresis area for the actuation with and without neutral string were 50 and 63 revolutions (shown with arrows) subsequently.

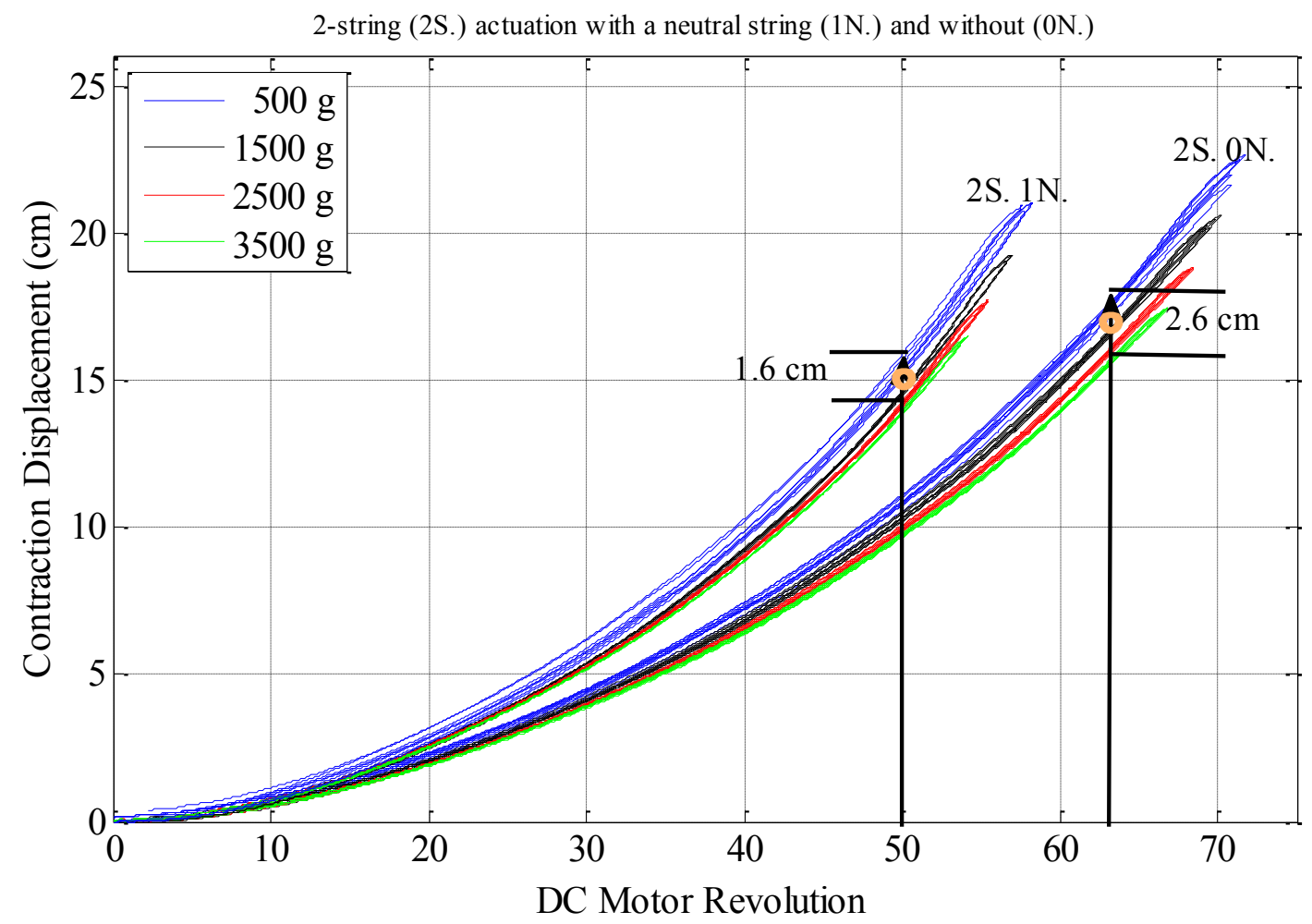

Figure 4. 18. Two-string actuation with a without a neutral string

As expected, adding a neutral string provided a $20 \%$ improvement in the actuation response for full contraction. Moreover, the maximum contraction of the average of four tests (shown with an orange dot) was decreased from $16.5 \mathrm{~cm}$ for the actuation without a neutral string to $15.5 \mathrm{~cm}$ with a neutral string (6\% reduction). The actuation without a neutral string demonstrated $2.6 \mathrm{~cm}$ 
of variation under various loads at maximum contraction, while the same configuration with a neutral string demonstrated $1.6 \mathrm{~cm}$ variation (38\% reduction).

The oscillatory motions were observed for both contraction and release. However, the oscillation was almost negligible in comparison with the response of the utility cable. Nonetheless, they were damped much faster for the configuration with a neutral string than without a neutral string for all loads. The oscillations of the two-string actuation without the neutral string for all loads were almost damped after the first 25 revolutions of contraction/release, which was the first $3-3.5 \mathrm{~cm}$ of the motion (the first $20 \%-25 \%$ of non-hysteresis contraction/release). However, the oscillation of the same configuration with a neutral string exhibited more precision in response. All oscillations for a two-string actuation with a neutral string were damped after the first 20 revolutions of the motion, which was the first $2.5-3 \mathrm{~cm}$ of non-hysteresis contraction/release ( $15 \%$ to $20 \%$ of the contraction/release).

\section{Two-string Actuation}

The responses of the three-string actuation with and without the neutral string are shown in Figure 4. 19. The maximum motor revolutions within the low-hysteresis area for the actuation with and without the neutral string were 42 and 51 revolutions (shown with arrows) respectively. As expected, adding a neutral string improved the actuation response for full contraction by $19 \%$.

Moreover, the maximum contraction of the average of four tests (shown with an orange dot) was decreased from $16.5 \mathrm{~cm}$ for the actuation without a neutral string to $13 \mathrm{~cm}$ with a neutral string (16\% reduction). The actuation without a neutral string demonstrated $1.1 \mathrm{~cm}$ error under various loads at maximum contraction, while the same configuration with a neutral string demonstrated $0.9 \mathrm{~cm}(18 \%$ reduction). 
The oscillatory motions were observed for both contraction and release. However, it was almost negligible in comparison with the response of the utility cable. Nonetheless, similar to the two-string actuation, they were damped faster with a neutral string than without a neutral string for all loads.

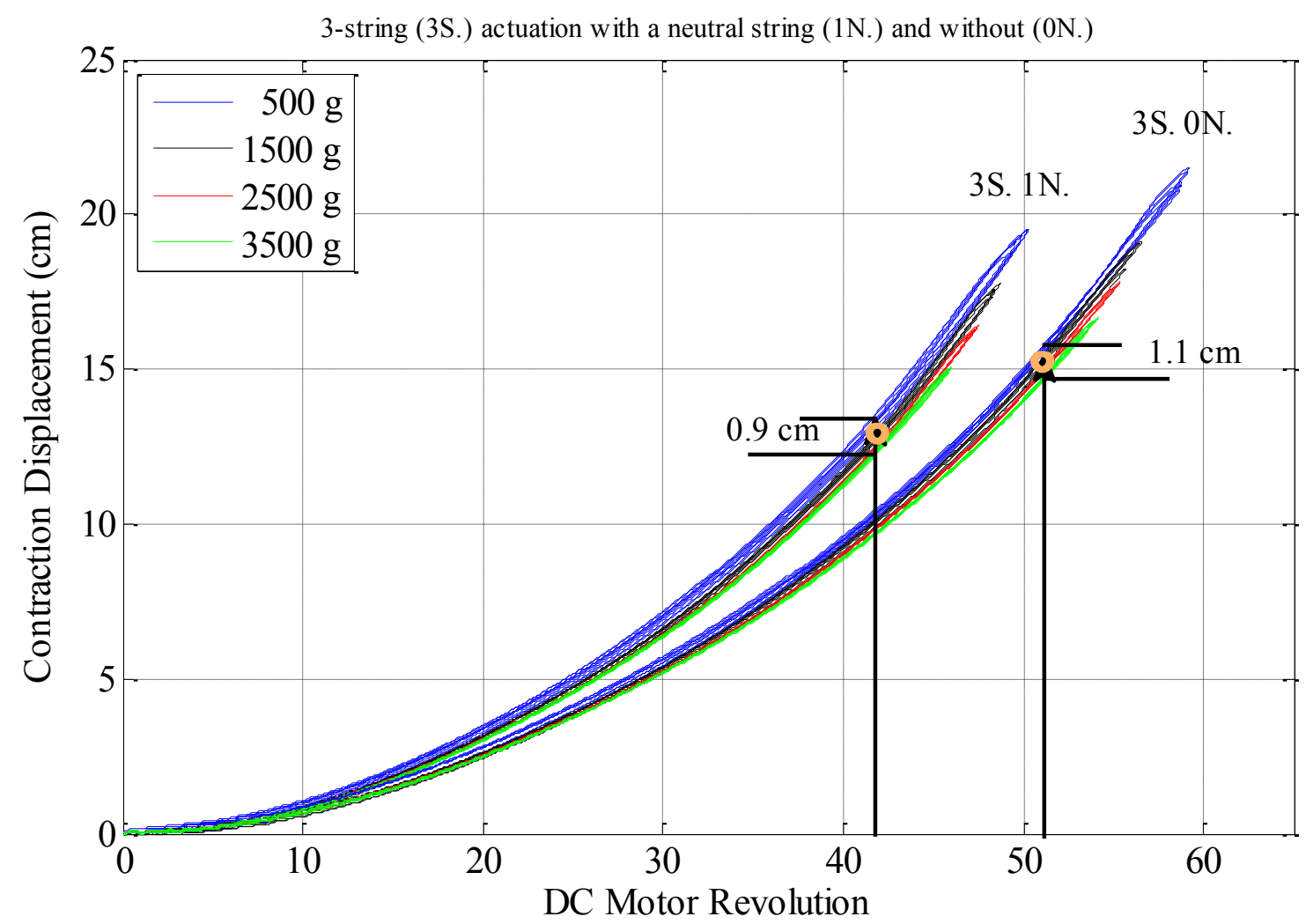

Figure 4. 19. Three-string actuation with a without a neutral string

The oscillations of the two-string actuation without the neutral string for all loads were almost damped after the first 15 revolutions of contraction/release, which was the first $1.5 \mathrm{~cm}$ of the motion (the first $9 \%$ of contraction/release). However, the oscillation of the same configuration with a neutral string exhibited more precision in response. All oscillations for a two-string actuation with a neutral string were damped after the first 10 revolutions of the motion, which was the first $1 \mathrm{~cm}$ of contraction/release (the first $7 \%$ of the non-hysteresis contraction/release). 


\section{Four, Five, and Six-string Actuation}

The responses of the actuation with four, five, and six effective strings and a neutral string are shown in Figure 4. 20. The maximum motor revolutions within the low-hysteresis area for the four, five, and six-string actuations were 36, 33, and 28 revolutions (shown with arrows) respectively.

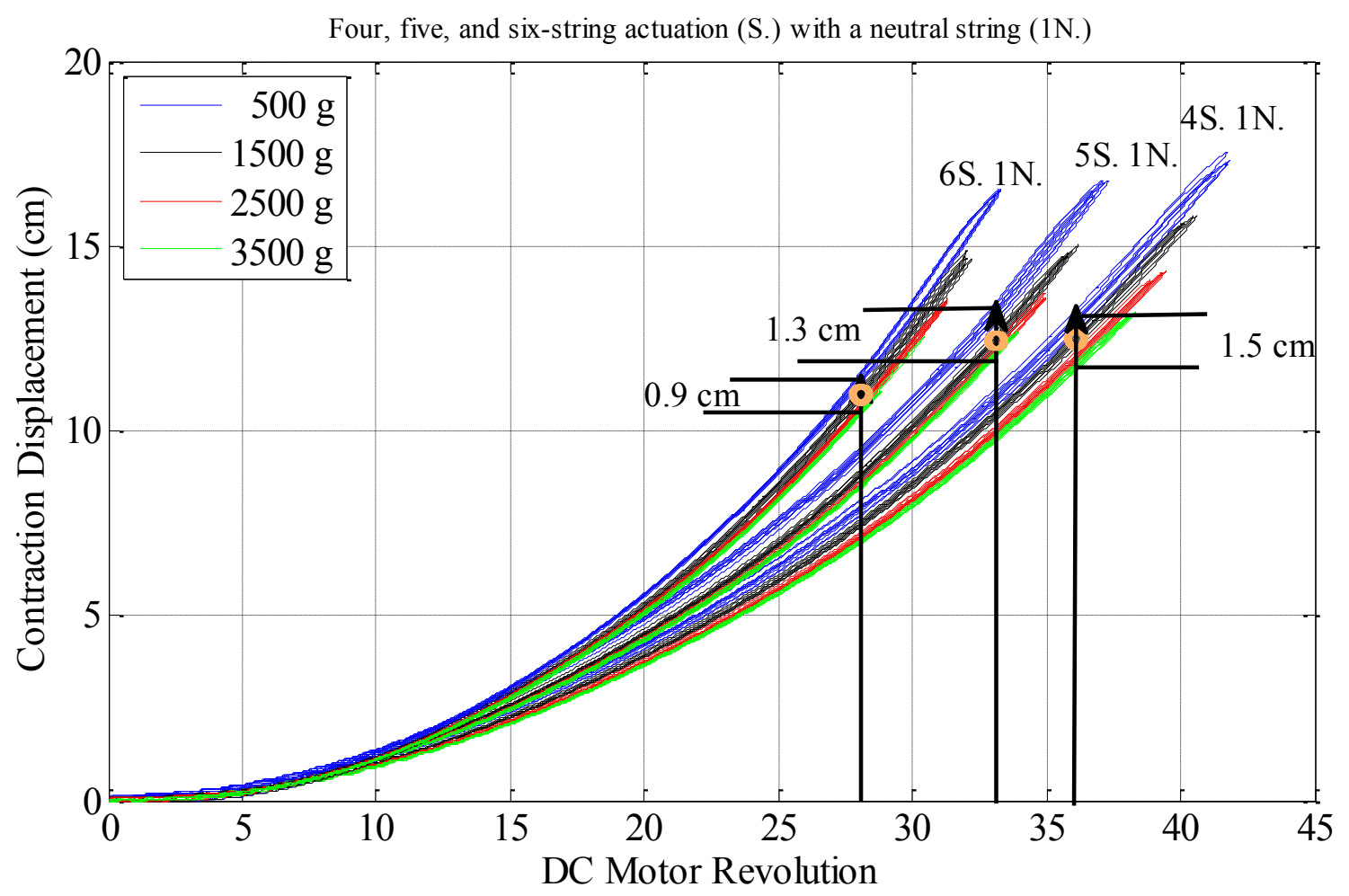

Figure 4. 20. Four, five, and six-string actuation with a neutral string

As expected, adding effective strings reduced the maximum average number of revolutions without going to the high-hysteresis area. The maximum contraction of the average of four tests (shown with an orange dot) were $12.5,12.5$, and $11 \mathrm{~cm}$ for the four, five, and six string configurations respectively. The maximum variation under various loads at maximum contraction were 1.5 for four-string, 1.3 for five-string, and $0.9 \mathrm{~cm}$ for six-string actuation. 
Similar to the previous configurations, all three of them exhibited some oscillatory response in contraction and release at low revolutions, which were damped in lower revolutions in comparison to the two and three-string configurations. The oscillations of the four, five, and sixstring actuations were damped after the first 5 revolutions of contraction/release respectively (the first $0.3-0.5 \mathrm{~cm}$ of contraction). This phase occupied almost the first $4 \%$ of the non-hysteresis contraction/release.

\section{Six-string Actuation}

The responses of the actuation with six effective strings, with and without a neutral string are shown in Figure 4. 21.

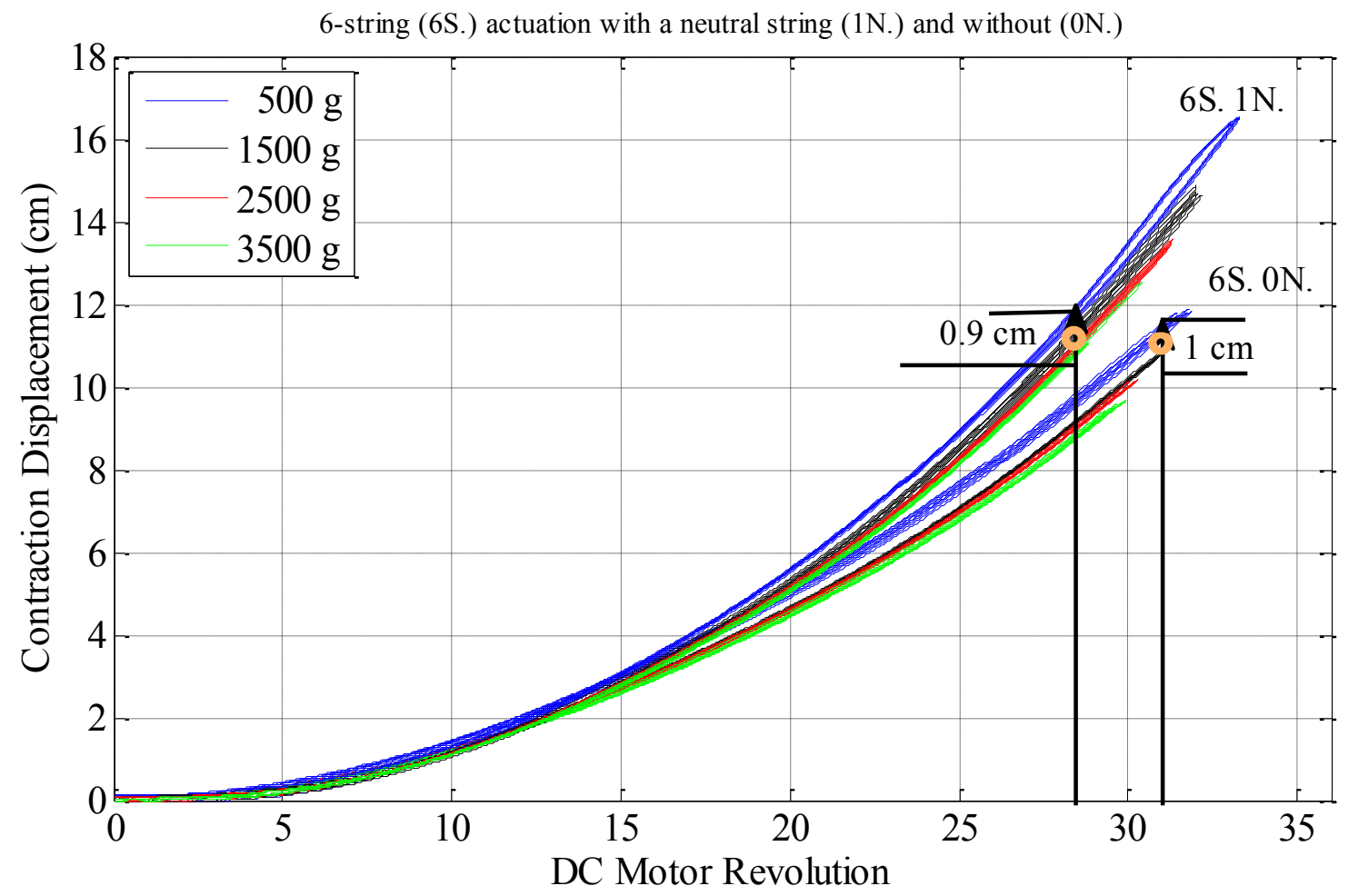

Figure 4. 21. Six-string actuation with a without a neutral string

The maximum motor revolutions within the low-hysteresis area for the actuation with and without the neutral string were 28 and 32 revolutions (shown with arrows) respectively. The 
maximum contraction of the average of four tests (shown with an orange dot) consequently will be $11 \mathrm{~cm}$ for both actuations with and without the neutral string. All oscillatory motions were damped after the first 5 revolutions of the motor (the first $0.5 \mathrm{~cm}$ of contraction).

\section{Discussion}

The summary of the characteristics of different actuations are listed below.

Table 4. 3. Characteristics of different configurations of the tactical cable strings

\begin{tabular}{lccccc} 
Actuation Type & $\begin{array}{c}\text { Maximum } \\
\text { DC Motor } \\
\text { Revolutions }\end{array}$ & $\begin{array}{c}\text { Maximum } \\
\text { Contraction } \\
(\mathrm{cm})\end{array}$ & $\begin{array}{c}\text { Maximum } \\
\text { Variation due to } \\
\text { Load }(\mathrm{cm})\end{array}$ & $\begin{array}{c}\text { Oscillatory } \\
\text { Contraction } \\
\text { Range }(\mathrm{cm})\end{array}$ & $\begin{array}{c}\text { Range of Contraction } \\
\text { without Oscillation } \\
(\mathrm{cm})\end{array}$ \\
\hline 2 effective, 0 neutral & 63 & 16.5 & 2.6 & $3.0-3.5$ & $13-13.5$ \\
\hline 3 effective, 0 neutral & 51 & 16.5 & 1.1 & 1.5 & 15 \\
\hline 2 effective, 1 neutral & 50 & 15.5 & 1.6 & $2.5-3.0$ & $12.5-13$ \\
\hline 3 effective, 1 neutral & 42 & 13 & 0.9 & 12 & $12-12.2$ \\
\hline 4 effective, 1 neutral & 36 & 12.5 & 1.5 & $0.3-0.5$ & $12-12.2$ \\
\hline 5 effective, 1 neutral & 33 & 12 & 1.3 & $0.3-0.5$ & 10.5 \\
\hline 6 effective, 0 neutral & 32 & 11 & 1 & 0.5 & $10.5-10.7$
\end{tabular}

As shown in Table 4. 3 adding a neutral string improved the oscillatory motion but not as significantly as the utility cable. It also decreased the maximum number of revolutions of the DC motor, which increased the contraction speed. The maximum non-oscillatory contraction was achieved using a two-string actuation without a neutral string with $13-13.5 \mathrm{~cm}$. However, the fastest response was achieved using a six-string actuation with a neutral string. In addition, increasing load increased the stability of the actuation and reduced the high-hysteresis range at high revolutions. 
To demonstrate the trend of change in the behavior of the actuation system by adding more strings, the responses under $3500 \mathrm{~g}$ mass load are shown in Figure 4. 22. Actuations with the same effective string configurations were colored similarly.

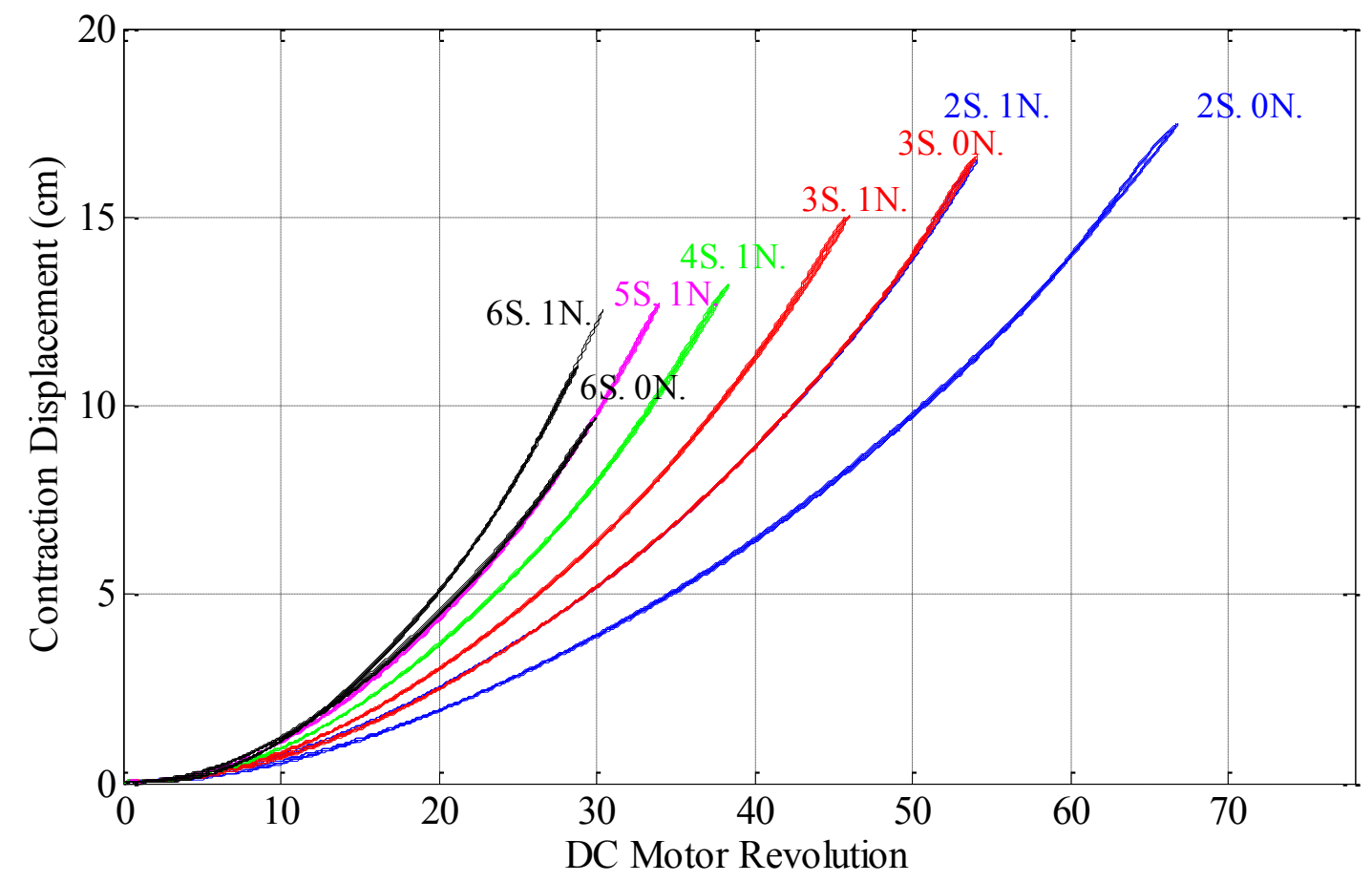

Figure 4. 22. The response of the actuation system under $3500 \mathrm{~g}$ mass load with different string-configurations

Similar to the heavy duty cable, tactical cable was tested under 200 cycles and the results are shown below.

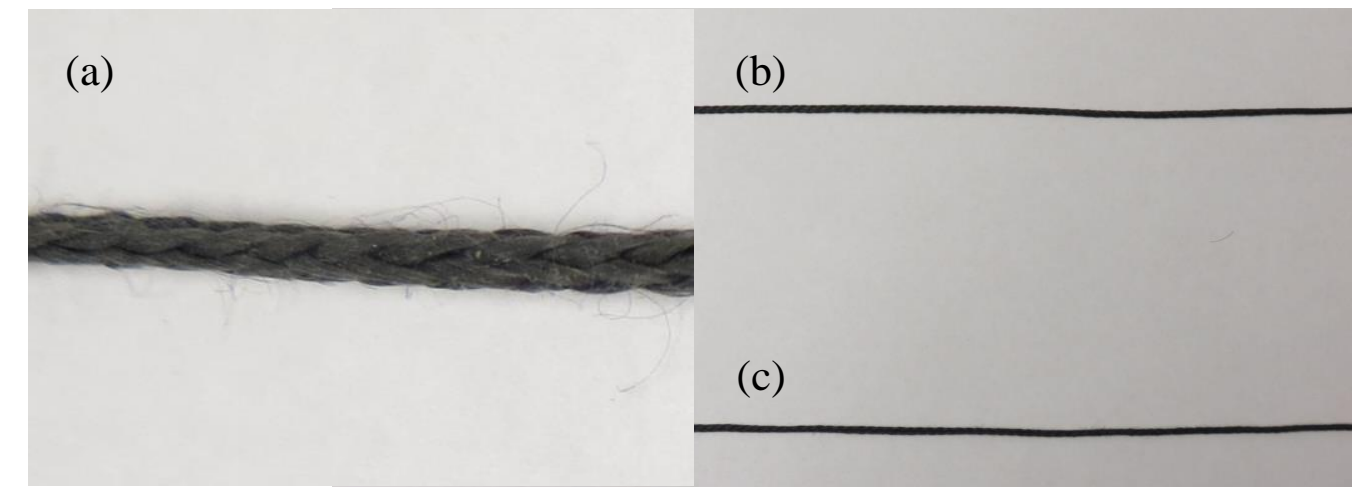

Figure 4. 23. (a) Worn out part of the sting (b) string after use (c) string before use 
As shown in Figure 4. 23, the string was not distorted at all after the work cycle. Moreover, since the string was abrasive resistive, the amount of abrasion was significantly less than the heavy duty cable.

\subsubsection{Nonlinear Area and its Effect on the Response of the System}

One interesting subject is the response of the actuation near the nonlinear area. This issue may be critical, if the pre-twisting bounds are close to this area. Thus there is a possibility that actuation enters this area in high speed motions.

Response of a two-string actuation without a neutral string using tactical cable was shown before. Three new tests with the same configurations were done in which the position of the DC motor was pushed further to investigate the behavior of the cable in the nonlinear range. Note that the nonlinear area presented in this section is different from the high-hysteresis area. Highhysteresis area is the part of the behavior of the actuation without going to the kinking mode. However, the nonlinear area covers the response of the actuation after going to the unpredictable nonlinear range. Low-hysteresis, high-hysteresis, and nonlinear areas are shown in Figure 4. 24.

Entering the nonlinear are not only generates huge hysteresis in the system, but also deviates the release response from the contraction response. In other words, the contraction trajectory is different from the release trajectory. Moreover, the contraction and release trajectories do not meet at the same split point. As shown in Figure 4. 24, the contraction response of the black line entered the nonlinear area at the onset of the nonlinear area. However, the release response of the same actuation met the contraction response at the onset of the high-hysteresis area. 


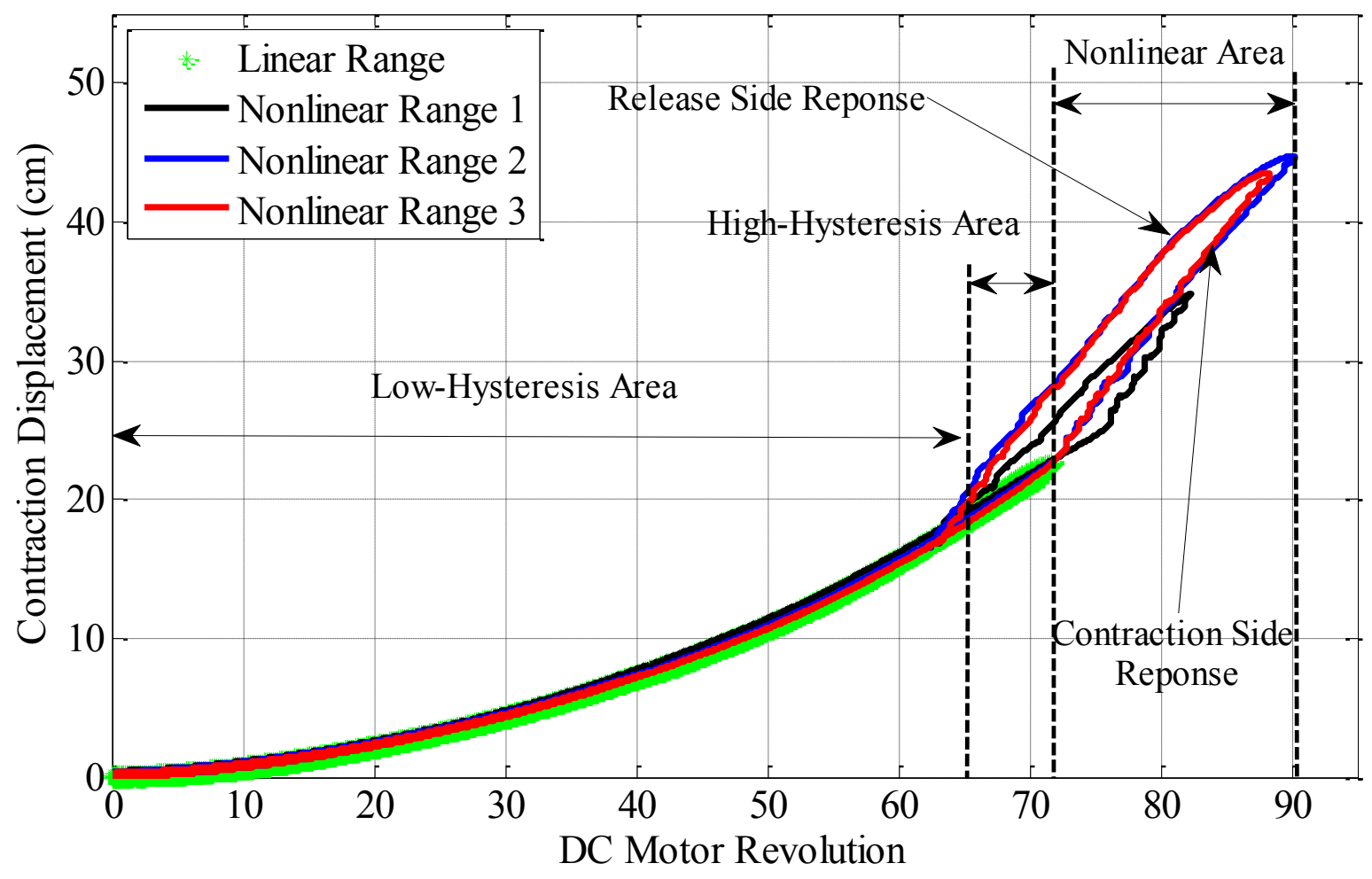

Figure 4. 24. The response of a 2-string actuation in linear and nonlinear range

It can be concluded from the response of a two-string actuation that going to the nonlinear area can significantly change the response of the system even in the high-hysteresis or potentially in the low-hysteresis area.

The physical geometry of the 2-string actuation at maximum linear mode and nonlinear mode are shown in Figure 4. 25. 


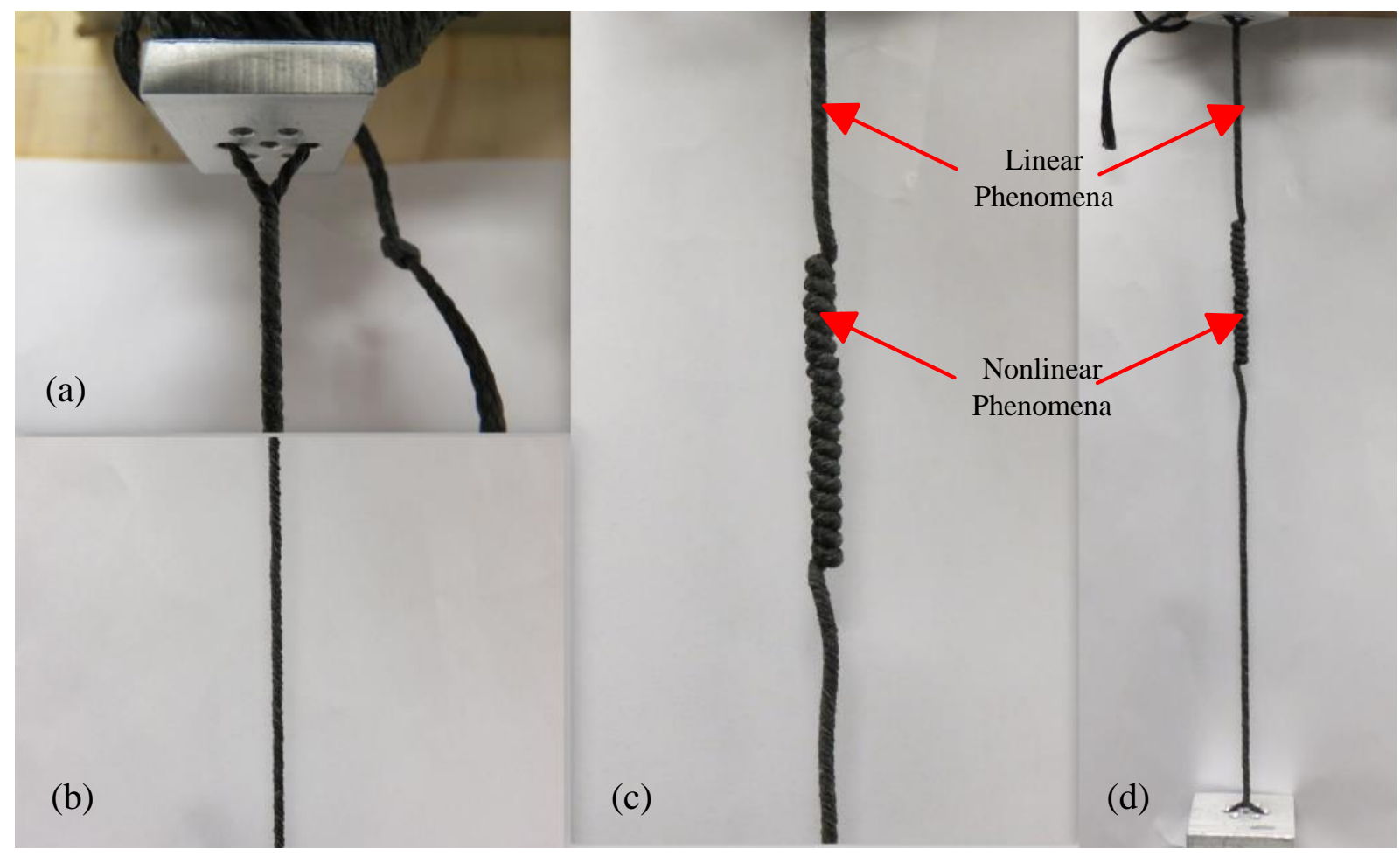

Figure 4. 25. Geormetry of (a) the string holder at maximum contraction without going to nonlinear area (b) 2string actuation at maximum contraction (c) and (d) (b) 2-string actuation at nonlinear area (kinking mode)

\subsubsection{Expected Error Analysis}

In this section the resultant error due to measuring components are provided and analyzed. There are two major sources of error: extensometer and DC motor encoder.

As discussed before the extensometer and DC motor encoder errors for the fishing line tests were $\pm 1 \mathrm{~mm}$ and $\pm 1 / 800$ revolution. Maximum contraction per revolution was $0.2 \mathrm{~cm}$ per revolution, which happened at 400 revolution (Figure 4. 8). Thus, the contraction error due to DC motor revolution is $( \pm 1 / 800) \times(0.2)= \pm 0.00025$ as listed below. 
Table 4. 4. Fishign line test errors

\begin{tabular}{cc} 
Extensometer error (mm) & Contraction error due to DC motor encoder (mm) \\
\hline $\pm 1 \mathrm{~mm}$ & $\pm 0.00025 \mathrm{~mm}$
\end{tabular}

Considering the two contraction error parameters the total error will be $\pm 1.00025 \mathrm{~mm}$.

The extensometer and DC motor encoder errors for the utility cable or tactical cable were \pm 0.2 $\mathrm{mm}$ and $\pm 1 / 128$ revolution. Maximum contraction per revolution was $0.97 \mathrm{~cm}$ per revolution, which happened at 32 revolution (Figure 4. 15 and Figure 4. 21). Thus, the contraction error due to DC motor revolution is $( \pm 1 / 128) \times(0.97)= \pm 0.0075$ as listed below.

Table 4. 5. Utility or tactical cable test errors

\begin{tabular}{cc} 
Extensometer error (mm) & Contraction error due to DC motor encoder (mm) \\
\hline $\pm 0.2 \mathrm{~mm}$ & $\pm 0.0075 \mathrm{~mm}$
\end{tabular}

Considering the two contraction error parameters of the utility or tactical cable tests the total error will be $\pm 0.2075 \mathrm{~mm}$. mentioned in Table 4. 6. As shown below both contraction error are negligible to the range of motion

Table 4. 6. Test errors

\begin{tabular}{cc} 
Fishign line test error $(\mathrm{mm})$ & Utility or tactical cable test error $(\mathrm{mm})$ \\
\hline $\pm 1.00025 \mathrm{~mm}$ & $\pm 0.2075 \mathrm{~mm}$
\end{tabular}




\subsubsection{Summary of Results and Analysis}

Three different strings were tested in this section: a monofilament fishing line, a heavy duty cable, and a tactical cable. Among all of them, the tactical cable had the best precision with the smallest amount of oscillatory motion. Moreover, it had the highest tolerable force and it was abrasion resistive.

There are several issues, which must be taken into consideration to implement the twisted string actuation, including 1) the number of strands within a set of strings and proper configuration of strings, and 2) the best string material-wise. Moreover the possible geometries of the strands should be taken into consideration if several strands required. Using several strands may result in different shapes of the strands in different situations [203], which can be the source of unexpected nonlinearity. The maximum number of strands with no neutral strand that maintains a stable shape in all angles is 3 (Figure 4. 7). To make the configuration stable for more than 3 strands and to increase the tolerable axial force, a neutral strand at the center of the other strands is needed. This string does not actively participate in actuation like other active strings (it gets twisted about itself), which may be a source of nonlinear behavior of the transmission.

Concerning the strand material, several criteria should be considered. Obviously, the maximum actuation force must not exceed the maximum tolerable force of the strand material. In addition, the friction of the sliding strands on each other due to motor rotation may abrade the outer surface of strands and result in rupture. For the repeated motion of a robotic device, fatigue cycles and the corresponding length change can also be an issue. These issues all need to be addressed prior to the commercialization of the assistive device. 


\subsection{Conclusion and Actuation Selection}

For the test device design, it was necessary to identify the transmission length of the twisted string required to achieve the targeted range of motion as well as the configuration and material of strings.

Based on the range of motion required for the elbow and shoulder joints, a proper configuration as well as string type should be selected. The actuation should satisfy the following requirements:

1. It should be able to provide enough actuation contraction and speed to perform tasks in the range of motion of the joints. Thus, the faster the actuation, the better it is.

2. It should provide the least contraction variation as the axis load increases. Thus, the more precise the behavior of the string, the simpler the control approach that can be used.

3. It should have the most possible linear response in the working range.

4. It is also necessary to avoid the high- $\alpha$ area (high revolutions) since high- $\alpha$ can yield a higher value of compound force $\left(n F_{i}\right)$.

Comparing Table 4. 2 and Table 4. 3, it turned out that the tactical cable had significantly less oscillatory response. Thus, it provided a bigger range of non-oscillatory contraction. Moreover, the hysteresis area at high revolutions was smaller for tactical cables and they were less sensitive to load change. Thus, tactical cable was chosen as the ultimate choice.

Minimum contraction variation against load was provided by a six-string actuation. Furthermore, this configuration provided the fastest response. Thus it will be the targeted actuation configuration. 


\section{Chapter 5: Control System}

\subsection{Introduction}

In this chapter all components of the system were linearized first and a mathematical model for the whole system was presented consequently. Then a control synthesis was made in order to perform an ADL. Ultimately results were presented and analyzed; and a conclusion was made.

In the first step all components of the system were identified as various sensors, number of actuator, actuation system, and etc. and a model were proposed for each part. Since the control architecture was based on the linear control systems, all components of the system was modeled first and then linearized. In the next step, a control synthesis for the upper arm robot was presented such that the robot could perform a predefined ADL. In order to perform the ADL task, two actuator for each joint were used, one for flexion and one for extension. Since the direction of actuation to perform flexion and extension motions were antagonistic, it was necessary to control the actuators such that they do not interfere. A master-slave control scheme was used to synchronize actuators. A test bed, which characterized the motion of the elbow joint, was designed 
in order to evaluate the controller performance. Accordingly, system criteria, which must be satisfied to provide a smooth motion were discussed. To evaluate the performance of the controller, the trajectory of the elbow joint was calculated according to the motion of the predefined ADL. According to the process, a PID controller was designed and results were presented and analyzed.

\subsection{Master-Slave Control}

Master-slave control methods have been investigated in the past 50 years [207]. This structure is still being used by researchers due to its simplicity, easy implementation, and flexibility to be merged with other control methods. Tsujiochi and colleagues [208] developed a pneumatic robot hand and designed a master-slave controller to follow the motion of the user. Contact force was used as the force feedback to regulate the inner pressure of the tubes. Hirai and etc. [209] designed a master-slave control for the Honda humanoid robot to grab objects. The robot was fully autonomous and based on the information receiving from the surrounding environment could find its path. A new anthropomorphic robot hand was designed by Mouri et al. [210], which employed a bilateral controller to control a five finger hand. Fingertip force and angle of joints were fed back to a master-slave controller. Tadano and Kawashimi developed a master-slave system with force sensing using a pneumatic servo system for laparoscopic surgery [211]. They designed a manipulator to support forceps, with $3 \mathrm{DOF}$, actuated by pneumatic cylinders. The master manipulator used a delta mechanism and a gimbal mechanism with a force sensor. The results showed that the operator could feel the force at the slave side to a satisfactory extent. Abolhassani has addressed a master-slave control of needle insertion in a Robotics-Assisted Prostate Brachytherapy [212]. He designed a master-slave set-up with multi-degrees of freedom and investigated the effect of force feedback on the accuracy of needle insertion. Tavakoli used a 
master-slave scheme to develop a haptics-based master-slave system for minimally invasive surgery [213] [214]. The communication and delay issues of the master-slave controller as well as implementation of a haptic force feedback were discussed. Huang and Kazerooni et al. employed a one DOF master-slave control to manage the Berkeley lower extremity exoskeleton [215]. They used a master leg of a user to control the robotic leg through position control. Rovers et al. [216] designed a robust master-slave controller for surgery applications, which utilized haptic sensors for force feedback. A Position Error architecture (PERR) and Kinesthetic Force Feedback architecture (KFF) were used, which enabled the surgeon to tele-operate in a more ergonomic environment

\subsubsection{Master-Slave Control Architecture [216] [217]}

Several bilateral control architectures could be proposed for a master-slave control scheme based on the type of sensory feedback and performance requirements. Major sensory feedbacks used for master-slave control can be categorized into two classes: position feedback through visual motion, angular, and linear sensors; and force feedback though gauge sensors and haptic interfaces. Some of the master-slave architectures are presented bellow:

\section{Position Error architecture (PERR)}

In this architecture, the position and force of the actuators are proportional to the position error between the master and slave. Since there is no force feedback between the master and slave, a high gain controller can ensure good performance; however, in high frequencies it makes the system instable. 


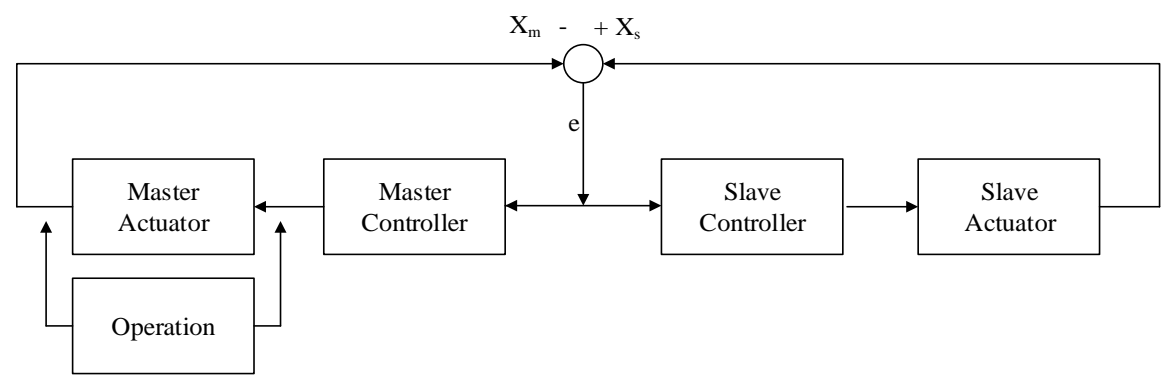

Figure 5. 1. PERR architecture

\section{Kinesthetic Force Feedback architecture (KFF)}

The kinesthetic force feedback architecture utilizes the force feedback of the slave actuator as the feedback command to the master actuator, while the master position is the reference command to the slave. This architecture, which is one of the best architectures for systems with antagonistic forces acting on each other, is sensitive to measurement noise.

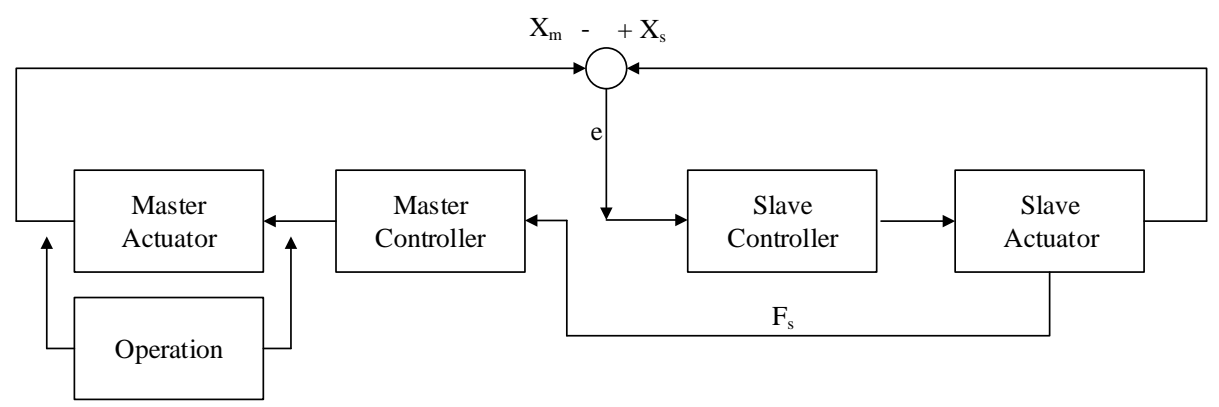

Figure 5. 2. KFF architecture

\section{Position and Force Feedback architecture (PFF)}

This architecture utilizes both force and position signals as feedback to the master. In other words, the feedback signal to the master is a linear combination of the position error of the master and slave (PERR) and the force measurement of the slave (KFF); however, the position error is still used to command the slave. This architecture has the characteristics of both PERR and KFF. Difficulties in implementation is a major drawback of this architecture. 


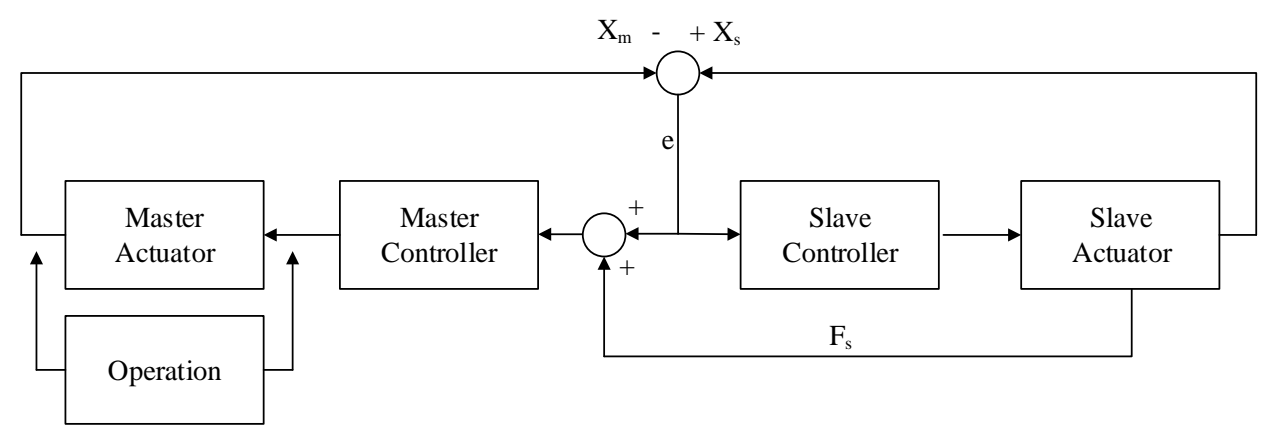

Figure 5. 3. PFF architecture

\subsubsection{Controller Architecture, and Analysis}

\section{System Modeling}

The master-slave architecture proposed to control the robot was similar to the PERR configurations with some modifications. The master and slave were pre-twisted to a level and then they followed the trajectory. Since the twisted string actuation method had non-linear behavior, the amount of contraction/release of the DC motors may not be equal for any given number of revolutions of the two motors. In other words, depending on how tightly strings are twisted, the resultant contraction might be different between the contracting and releasing actuators for the same number of revolutions of the DC motors. Thus, it needed special consideration to determine the trajectory of the slave actuator according to the amount of contraction/release that the master provided. The angle trajectory, which was the angle of the pulley in degrees, was predefined and the master should follow that. The angle was the result of the contraction and release of the master and slave, while slave followed the rotational position of the master. Since the actuation has a non-linear behavior, an extra block (actuation approximator block) approximated the corresponding position of the slave such that it provided the same contraction that the master was providing. Due to changing inertia of the system (caused by acceleration or variation of the torque of joints), slave 
may encounter disturbances, which could change the actuation required to meet the desired contraction. Thus, the tracking error of the slave was feed back to the master to slow down the master in case the slave can't overcome the disturbance. To ensure that the targeted angle of the elbow was achieved, an angular encoder was mounted on the pulley. Moreover, to capture the value of tracking error, a linear encoder was used.

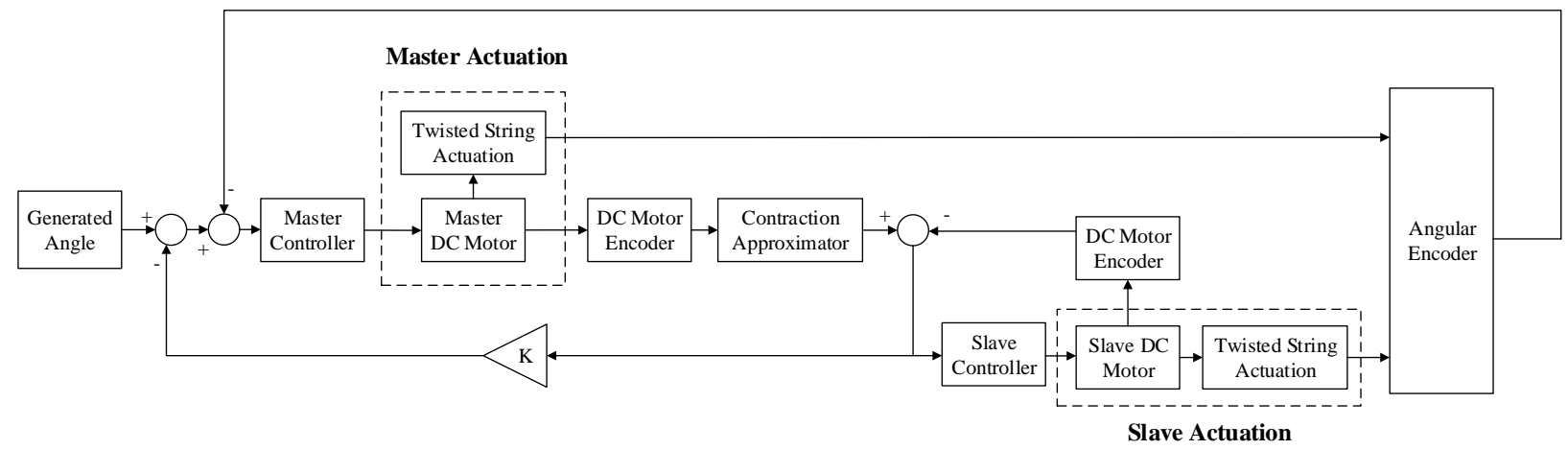

Figure 5. 4. Schematic of the master-slave control

To design a PID controller, master and slave were treated as independent systems and the feedback from one to other was modeled as a disturbance. The separated model of the master and slave are shown below.

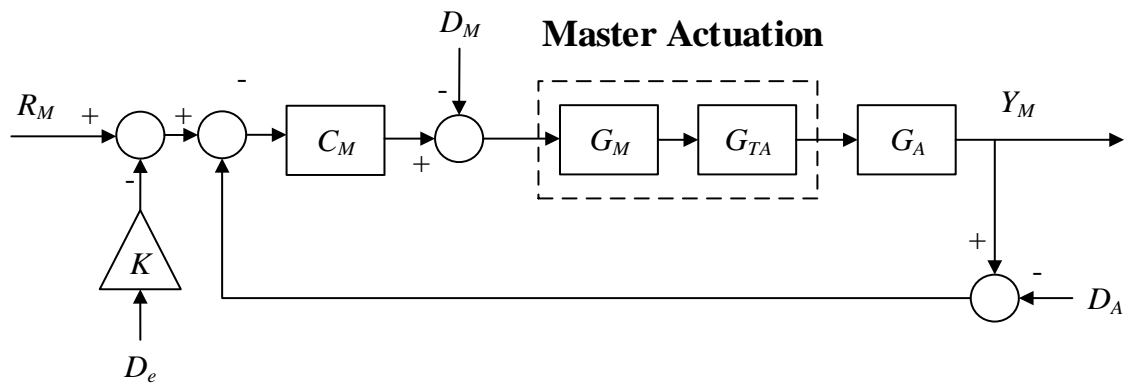

Figure 5. 5. Master model 


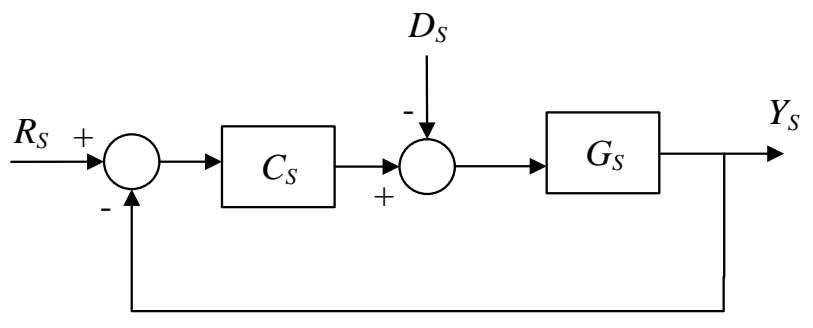

Figure 5. 6. Slave model

where the symbols are defined in Table 5. 1.

Table 5. 1. Table of symbols

\begin{tabular}{ll|ll}
$R_{M}$ & Master position trajectory & $G_{T A}$ & Twisted actuation transfer function \\
\hline$R_{S}$ & Slave position trajectory & $G_{A} \quad$ Angular encoder transfer function \\
\hline$C_{M}$ & Master controller transfer function & $D_{A} \quad$ Angular disturbance generated by slave \\
\hline$C_{S} \quad$ Slave controller transfer function & $D_{e} \quad \begin{array}{l}\text { Disturbance generated by the error of the slave } \\
\text { trajectory and the desired trajectory }\end{array}$ \\
\hline$G_{M} \quad$ Master actuator transfer function & $D_{M} \quad$ Disturbance on the master \\
\hline$G_{S}$ & Slave actuator transfer function & $D_{S}$ & Disturbance on the slave \\
\hline$K$ & $\quad$ Slave feedback error gain & $H_{E}$ & Transfer function of the DC motor encoder
\end{tabular}

The output of the master is

$$
Y_{M}=\frac{G_{M} G_{T A} G_{A}}{1+C_{M} G_{M} G_{T A} G_{A}}\left(C_{M} R_{M}-K C_{M} D_{e}+C_{M} D_{A}+D_{M}\right)
$$

For further simplification, the transfer function of the master system was defined as:

$$
G_{M s y s}=G_{M} G_{T A} G_{A}
$$

Thus, the transfer function of the output of the master for three disturbances and the input trajectory are 


$$
\begin{gathered}
G_{R}=\frac{Y_{M}(s)}{R_{M}(s)}=\frac{C_{M} G_{M s y s}}{1+C_{M} G_{M s y s}}, \\
G_{e}=\frac{Y_{M}(s)}{D_{e}(s)}=\frac{-K C_{M} G_{M s y s}}{1+C_{M} G_{M s y s}}, \\
G_{A}=\frac{Y_{M}(s)}{D_{A}(s)}=\frac{C_{M} G_{M s y s}}{1+C_{M} G_{M s y s}}, \\
G_{M}=\frac{Y_{M}(s)}{D_{M}(s)}=\frac{G_{M s y s}}{1+C_{M} G_{M s y s}},
\end{gathered}
$$

Using the same method, the output of the slave as a function of disturbance and input is

$$
Y_{S}=\frac{G_{S}}{1+C_{S} G_{S}}\left(C_{S} R_{S}-D_{S}\right)
$$

\subsection{Component Models, Actuation Selection and Identification}

In this section the whole actuation system including actuator, actuation, and sensors were modeled by a single transfer function. For this purpose, the transfer function of the actuator was identified first. Based on the required range of motion, the contraction/release trajectory of the selected actuation was linearized by a transfer function. As the last step, angular encoder was modeled by a transfer function. Knowing all three transfer functions, transfer function of the system with voltage as input and angular motion as output was proposed.

\subsubsection{Motor Modeling and Identification [218] [219]}

DC motors are among the commonly used actuators. They provide excellent speed control for acceleration and deceleration and the torque control is fairly simple. It allows precise voltage 
control since its power supply connects directly to the field of the motor. This characteristic is necessary in speed and torque control applications. The open-loop transfer function of the DC motor will be:

$$
G_{D C}(s)=\frac{\dot{\Theta}(s)}{V_{D C}(s)}=\frac{K_{e m f}}{(J s+b)(L s+R)+K_{e m f}^{2}}\left(\frac{\mathrm{rad} / \mathrm{sec}}{\text { volts }}\right),
$$

where $J$ is the moment of inertia of the rotor, $b$ is the motor viscous friction constant, $R$ is the electric resistance of the circuit, $L$ is the electric inductance of the circuit, and $K_{\text {emf }}$ is the back emf

constant. $\dot{\Theta}(s)$, as the output is the speed of the motor. The position of the DC motor as a function of the voltage input can be obtained by adding an integrator.

\section{Motor Test and Model Validation}

To do the experiment a high-torque DC motor with 19:1 gear ratio and 64 counts per revolution (CPR) encoder was selected. To validate the model of the DC motor a biased chirp signal with 17 volts bias and 2 volts amplitude ranging from $0.1 \mathrm{~Hz}$ to $10 \mathrm{~Hz}$ was generated. The mean (17 volts) was an arbitrary number. It should be noted that if the mean input voltage drops 12 volts, the response of the DC motor becomes too slow to capture its characteristics.

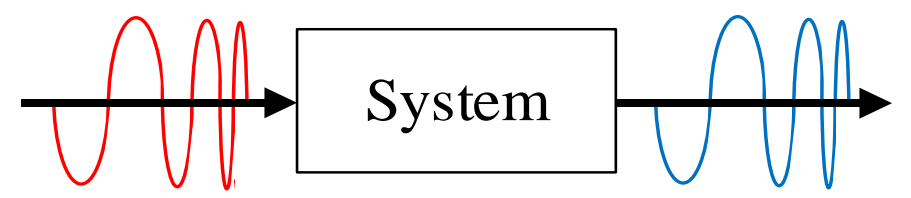

Figure 5. 7. Input and outputs of the system using Chirp signal

The data was obtained and the second-order transfer function was approximated by a firstorder one. The input and output of the DC motor as well as the response of the approximated transfer function are shown in Figure 5. 8. 

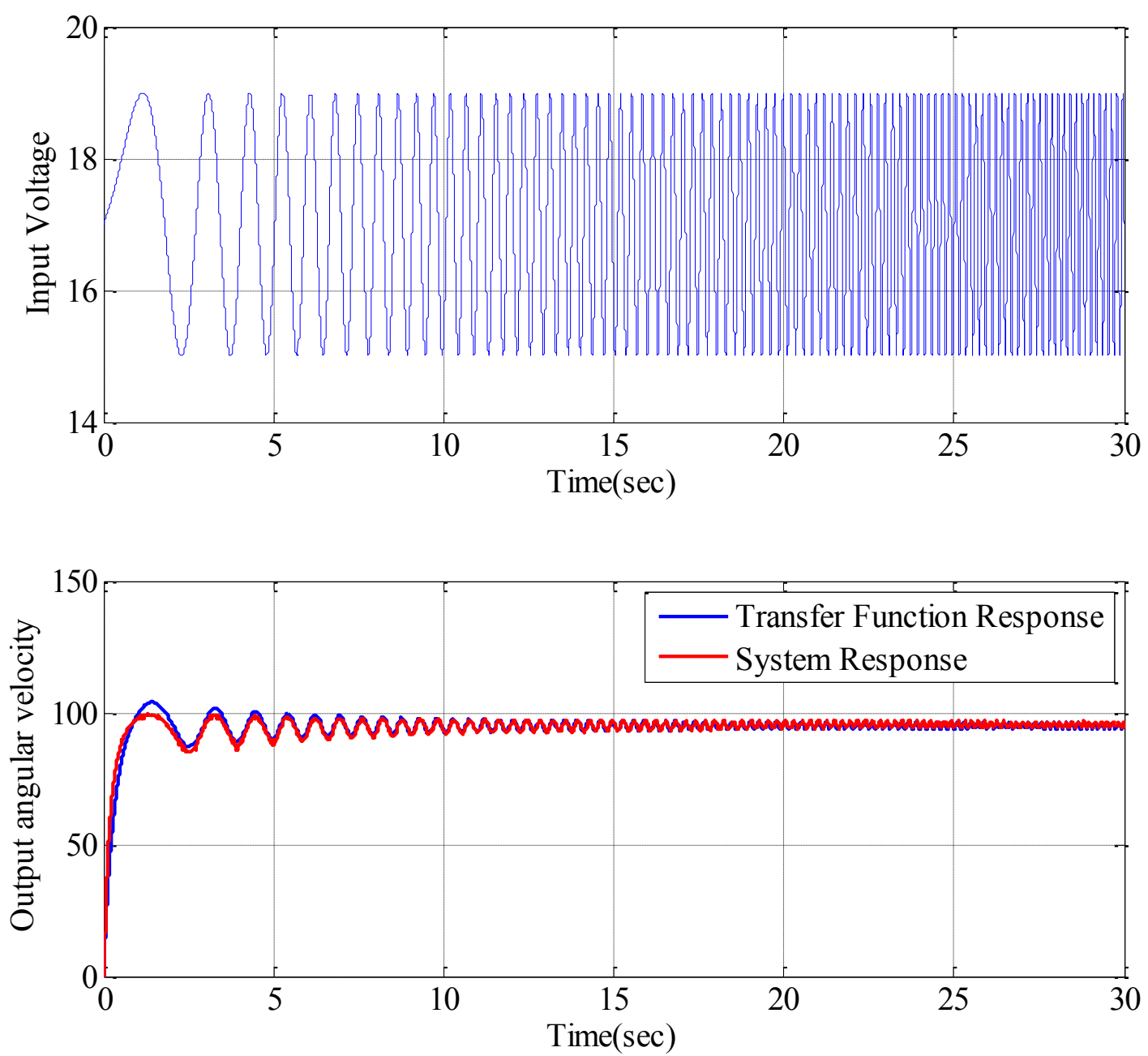

Figure 5. 8. Input and output of the approximated transfer function

$$
G_{D C}(s)=\frac{K_{D C}}{T_{D C} s+1}\left(\frac{\mathrm{rad} / \mathrm{sec}}{\text { volts }}\right),
$$

and the value of $K_{D C}$ and $T_{D C}$ are shown in Table 5. 2.

Table 5. 2. Values of the transfer function parameters

\begin{tabular}{cc}
$K_{D C}$ & $T_{D C}$ \\
\hline 5.57 & 0.24
\end{tabular}




\section{Master and Slave Actuator Model}

The transfer function of the DC motor was identified before as $G_{D C}$, in which voltage was the input and velocity in Radians/second was the output. The block diagram, however, was built around position control, so $G_{D C}$ must be multiplied by a factor to translate radians to revolutions, and an integrator to translate velocity to position in order to generate master or slave transfer function. Thus, the transfer function of the master or slave is

$$
G_{M}(s)=\left(\frac{K_{D C}}{T_{D C} s+1}\right)\left(\frac{K_{\mathrm{Rev}}}{s}\right) \quad(\mathrm{Rev} / \text { Volts }),
$$

where $K_{D C}$ and $T_{D C}$ were identified before as 5.569 and 0.24003 respectively, and $K_{\mathrm{Rev}}$, as the factor translating radians to revolution was $1 / 2 \pi$. Thus, the actuator transfer function is

$$
G_{M}(s)=\frac{0.8863}{s(0.24003 s+1)} \quad(\operatorname{Rev} / \text { Volts }),
$$

\subsubsection{Twister String Actuation Converter}

To acquire the transfer function of the twisted actuation and ensuring the consistency of the behavior of the actuation, it was tested while the actuation (strings with a proper configuration) was mounted on the actuator (on the test bed). 


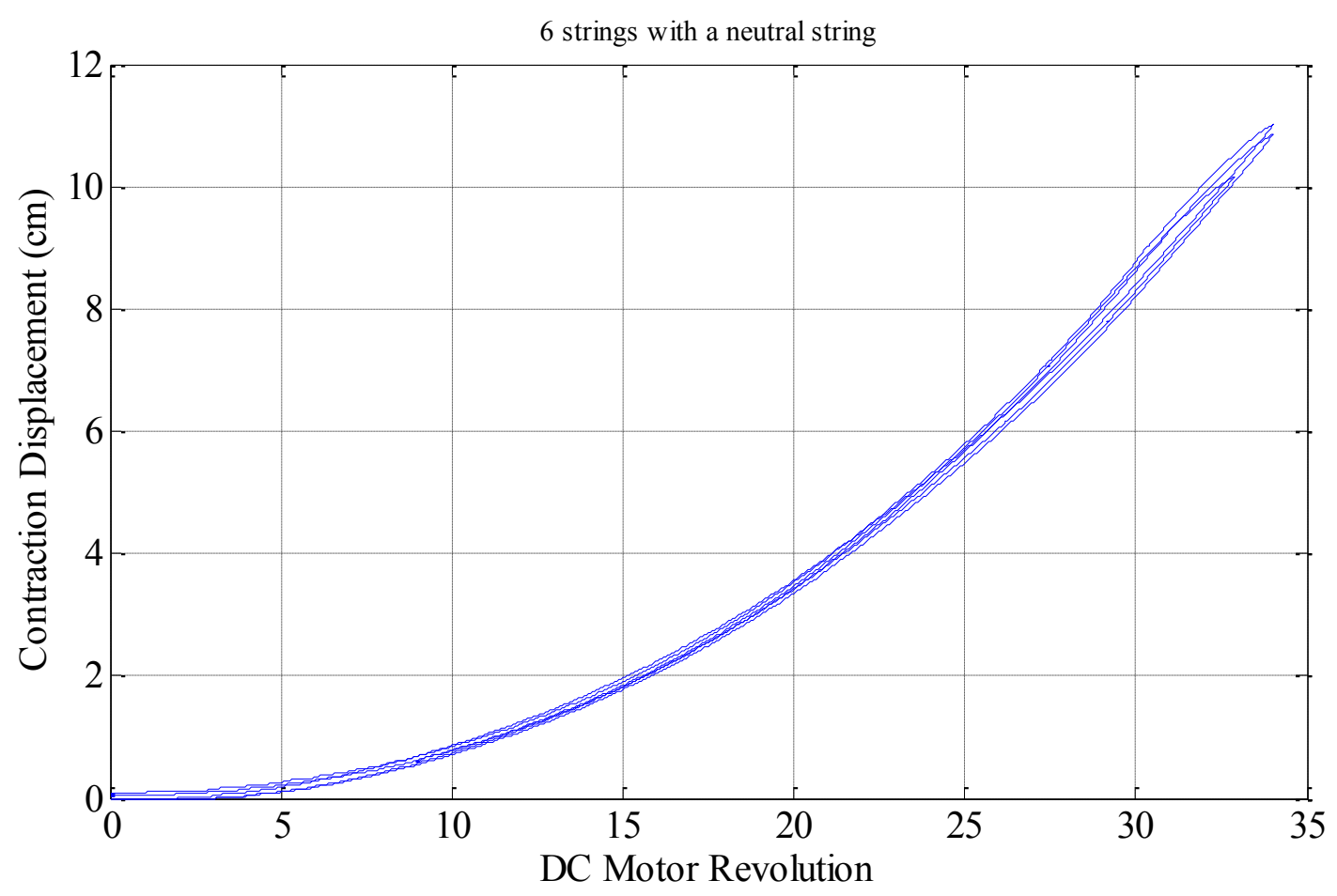

Figure 5. 9. Behavior of the actuator with 6 active strings and one neutral string

Since the twisted actuation had nonlinear behavior, its response must be linearized as a ratio of revolution to contraction. Using the radius of the pulley mounted on the test bed $(4 \mathrm{~cm})$ the contraction range for full motion ( 0 to $135^{\circ}$ flexion and extension) of the elbow was calculated as $9.42 \mathrm{~cm}$.

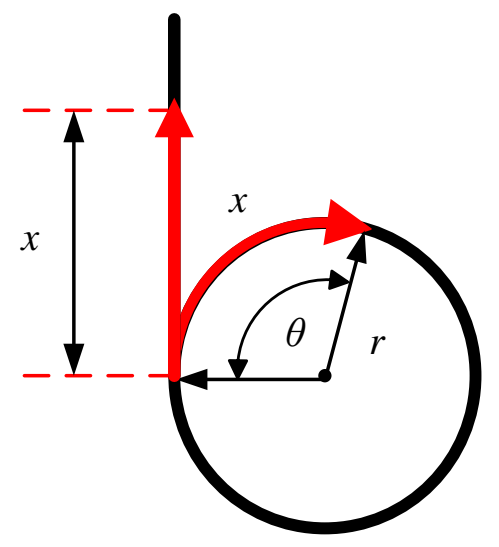

Figure 5. 10. Conversion from contraction to angle 
Thus, the elbow assistance robot should operation in this range. Since the response of the actuation was very slow in low revolutions, actuation must be pre-twisted. Thus, the master was pre-twisted for 11 revolutions $(1 \mathrm{~cm})$ and the slave for 33 revolutions $(11 \mathrm{~cm})$. The linearized range is shown in Figure 5. 11.

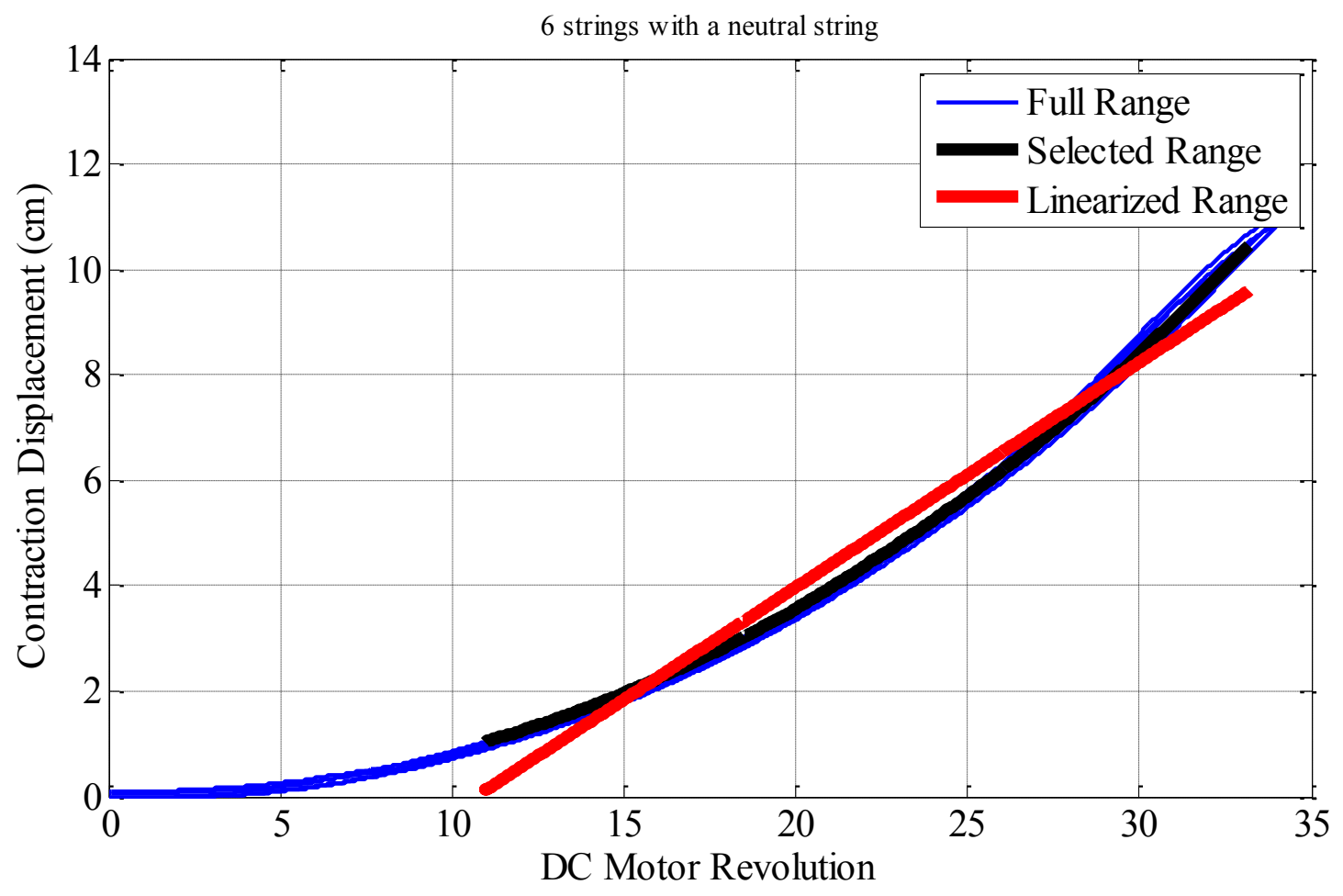

Figure 5. 11. Linearized range of motion

The slope of the linearized line was 0.426 , which will be the linearized transfer function of the twisted string actuation $\left(G_{T A}\right)$.

$$
G_{T A}(s)=0.426,
$$




\subsubsection{Angular Encoder}

Angular encoder was modeled as a ratio too, which translated the contraction of the actuation to the resultant angle. Knowing the radius of the pulley the gain is

$$
G_{A}(s)=\frac{\theta}{x}=\left(\frac{180}{\pi}\right) \frac{1}{r} \quad(\text { degrees } / \mathrm{cm}),
$$

where $r, x$, and $\theta$ are the radius of the pulley, contraction in $\mathrm{cm}$, and angle in degrees respectively. Knowing the radius of the pulley to be $4 \mathrm{~cm}, G_{A}$ as a gain is 14.32 .

$$
G_{A}(s)=14.32
$$

Knowing $G_{M}, G_{T A}$, and $G_{T A}$ the transfer function of the master actuation is:

$$
G_{M s y s}=G_{M} G_{T A} G_{A}=\frac{5.4067}{s(0.24003 s+1)}
$$

\subsection{Test Bed Design and Experimental Procedures}

The two actuations used to control the elbow joint are shown in Figure 5. 12. To ensure that the two actuations do not conflict each other, they should provide the same contraction/release. 


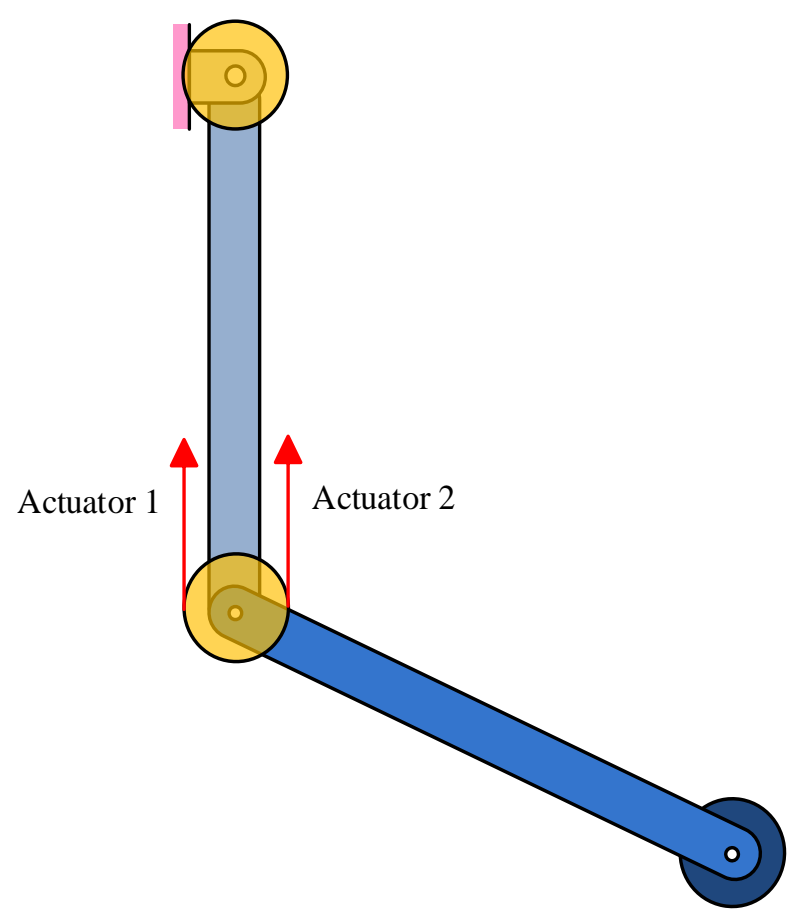

Figure 5. 12. Schematic of the actuation acting on the robot arm

To capture if two actuators are conflicting or not a test bed was designed. Two actuators were attached to two sides of a pulley, which was mounted on a slider. As one actuator contracted, the other one released. If the amount of contraction provided by one was equal to the amount of release provided by the other, master and slave were considered synchronized. Otherwise the pulley would be pulled up or down, which demonstrated that the master-slave tracking was not good.

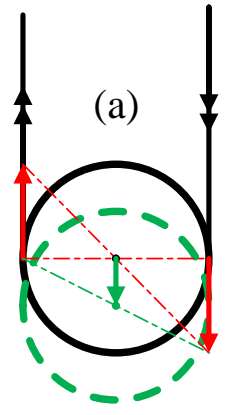

(b)

Figure 5. 13. Schematic of the test bed when (a) the left actuator contracts and the right actuator releases, (b) only the right actuator releases (error due to conflict is marked in green) 
To capture this upward/downward motion an extensometer with $0.2 \mathrm{~mm}$ resolution was used. The $x P C$ Target toolbox of Matlab (2013a) was used to implement the controller. $x P C$ Target is a real time control toolbox in which the digital controller is implemented using a desktop computer instead of a microcontroller.

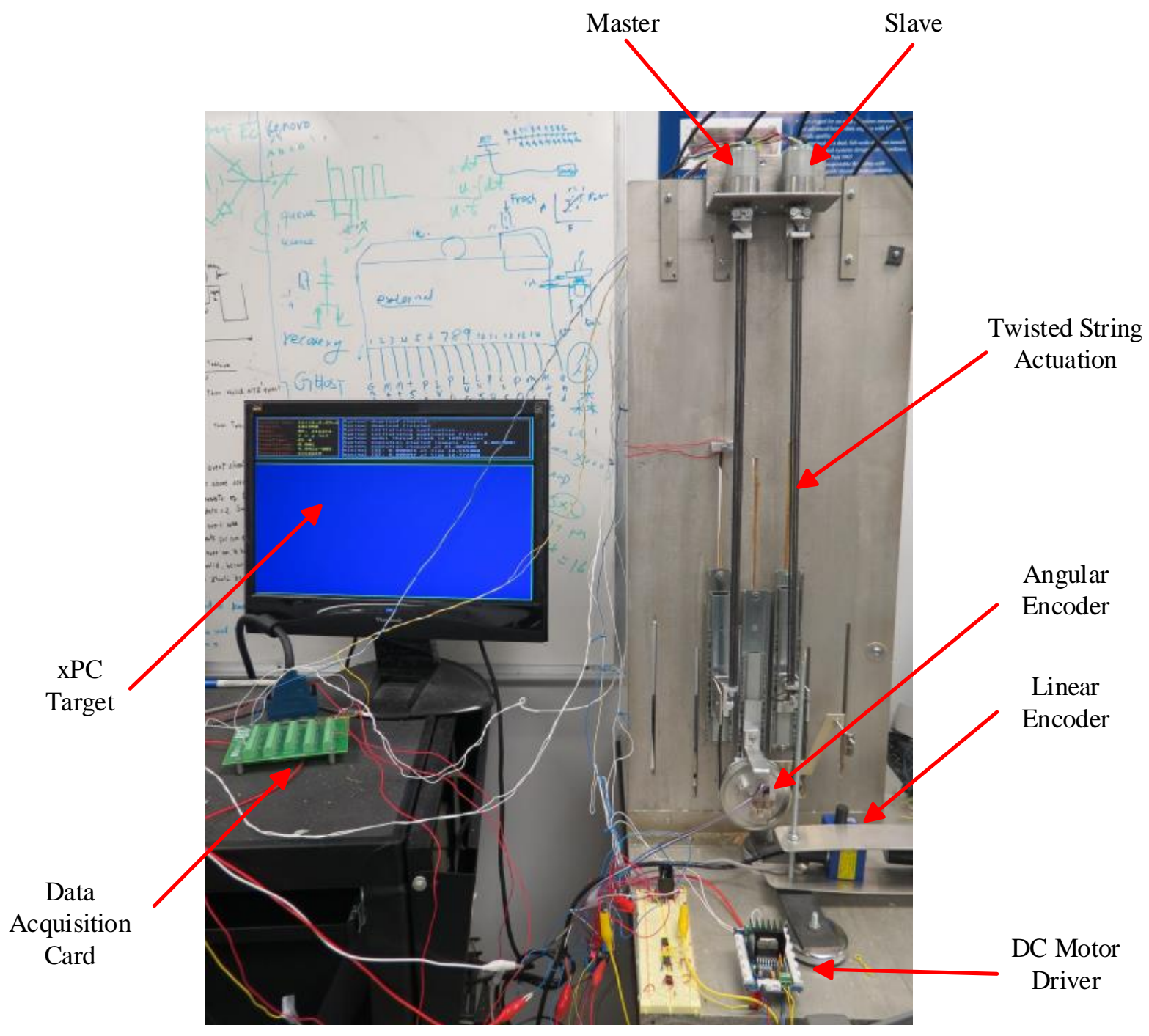

Figure 5. 14. Control test bed 
To acquire data from the sensor including the two motor encoders and the linear encoder, two data acquisition cards (National Instruments PCI-6221 and National Instrument PCI-6601) were used, which communicated with the desktop computer in real-time. To use $x P C$ Target, it was necessary to model the whole system is Simulink and then compile the file to $\mathrm{C}$ and upload it on the desktop computer.

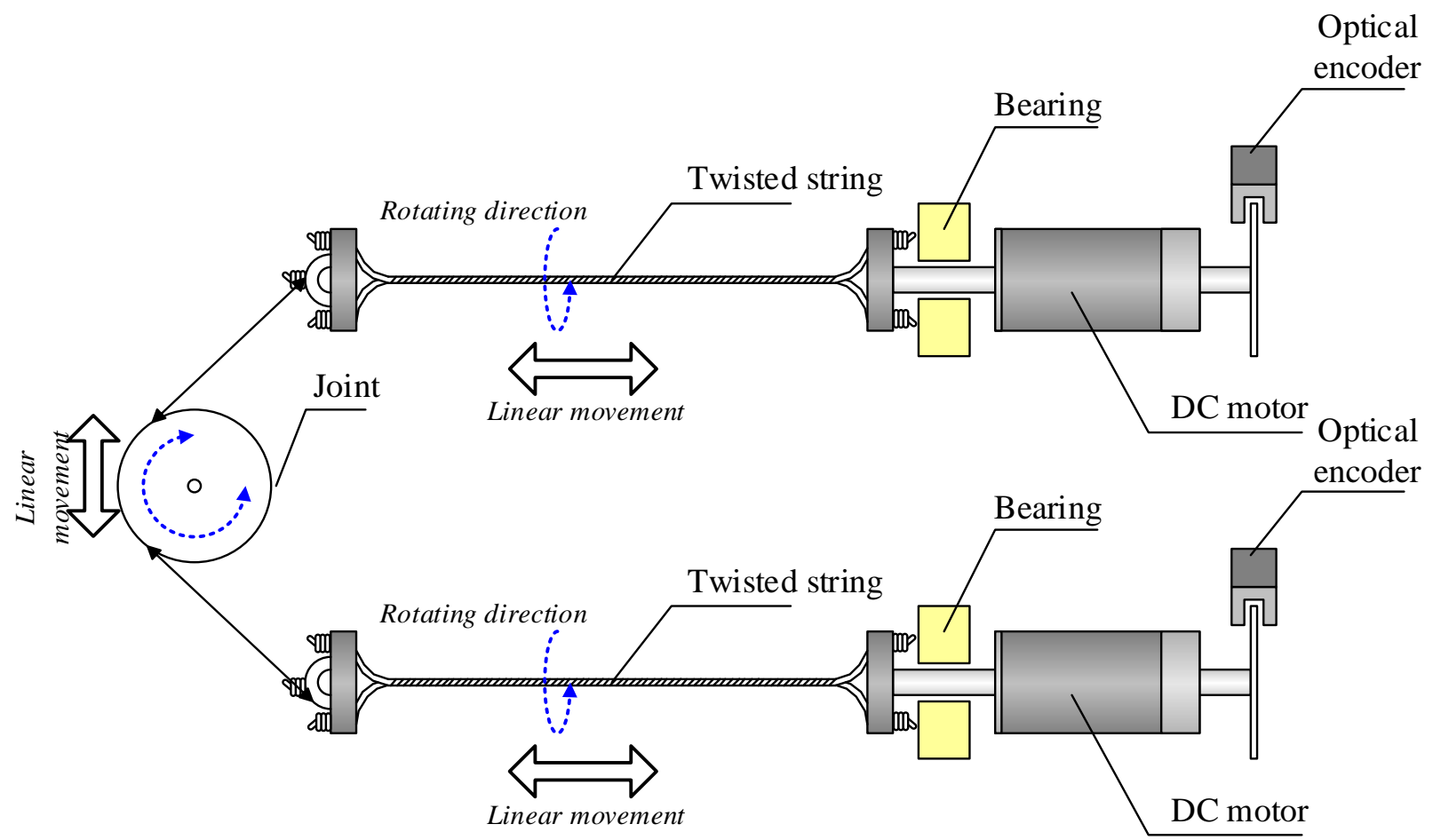

Figure 5. 15. Skematic of the twisted string actuation once mounted on the test stand

\subsection{Root Locus Analysis, Gain Selection, and Analysis}

Two PID controllers in a parallel configuration were used as the controller. One of the drawbacks of using and ideal PID is that the derivative term with high gains are more susceptible to high frequency measurement noise. This means that large variations in control effort signal will be generated by high-frequency measurement noise [220]. To increase the robustness of the controller 
at high frequencies, a first-order filter with constant $N$ was used. The transfer function of the PID control is shown below:

$$
G_{c}=K_{P}+K_{I} \frac{1}{s}+K_{D} \frac{N}{1+N \frac{1}{s}}
$$

The derivative transfer function at low frequencies would be $s K_{D} N$, which leads to zero. However, at high frequencies it would be bounded to $K_{D} N$. Thus, the closed form of the PID controller would be:

$$
G_{c}=\left(K_{P}+K_{D} N\right) \frac{s^{2}+\left(\frac{K_{P} N+K_{I}}{K_{P}+K_{D} N}\right) s+\frac{K_{I} N}{K_{P}+K_{D} N}}{s(s+N)},
$$

This formula can be simplified as:

$$
\begin{gathered}
G_{c}=K_{P I D} \frac{\left(s-z_{1}\right)\left(s-z_{2}\right)}{s(s-p)}, \\
K_{P I D}=K_{P}+K_{D} N,
\end{gathered}
$$

where $K_{P I D}$ is the new gain of the controller. This form of controller adds a pole at zero and one far away from the origin. The higher the filtering factor $N$, the bigger the pole will be, which makes the system more susceptible to high frequency noise but further stabilizes the closed-loop system. Whereas, ideal PID without noise attenuation, only adds a pole at the origin. Moreover, it adds two zeros at $z_{1}$ and $z_{2}$.

The other drawback of the PID control is integrator windup [220]. Although the controller is according to linear control theory, expected or unexpected non-linear phenomena can happen. Any mechanical component in a system has physical limitations, such as the maximum speed of a DC motor, and if the controller reaches the limitation, the feedback is broken. Since the DC motor is saturated, the system runs as an open-loop system. If the controller is designed with an integrator, 
the error during saturation will be integrated. When it happens, the accumulated error of the integrator term will become so big such that it needs a long time to "unwind" once the sign of the error changes. The result is overshoot and oscillation. There are various anti-windup algorithms such as avoiding saturation, conditional integration, back-calculation, proportional band, and etc.

For this purpose conditional integration or integral clamping was used. This method, which is an alternative to back-calculation or tracking techniques is based on switching the integrator off when it is far away from the steady state. In other words, the integrator is turned on only when some criteria are fulfilled. Otherwise the integrator part is treated as a constant. In this method the saturation band of the actuator must be defined.

The selected cable for the actuation was the tactical cable since it provided the most stable response with least vibration. The selected configuration had 6 active strings and one neutral string. The selection of the configuration was based on the speed of the actuation as well as stability. This configuration had the least oscillatory motion and very consistent response. Moreover, it could achieve the highest contraction with the minimum number of DC motor rotations, which means that it provided the fastest response.

\section{Master PID Tuning}

To tune the controller, the root locus method was used. Poles and zeros of the controller were set such that it satisfied the desired requirements of 5\% overshoot (or 0.7 damping ratio) and 0.6 seconds settling time.

The root locus of the master actuator is shown in Figure 5. 16: 


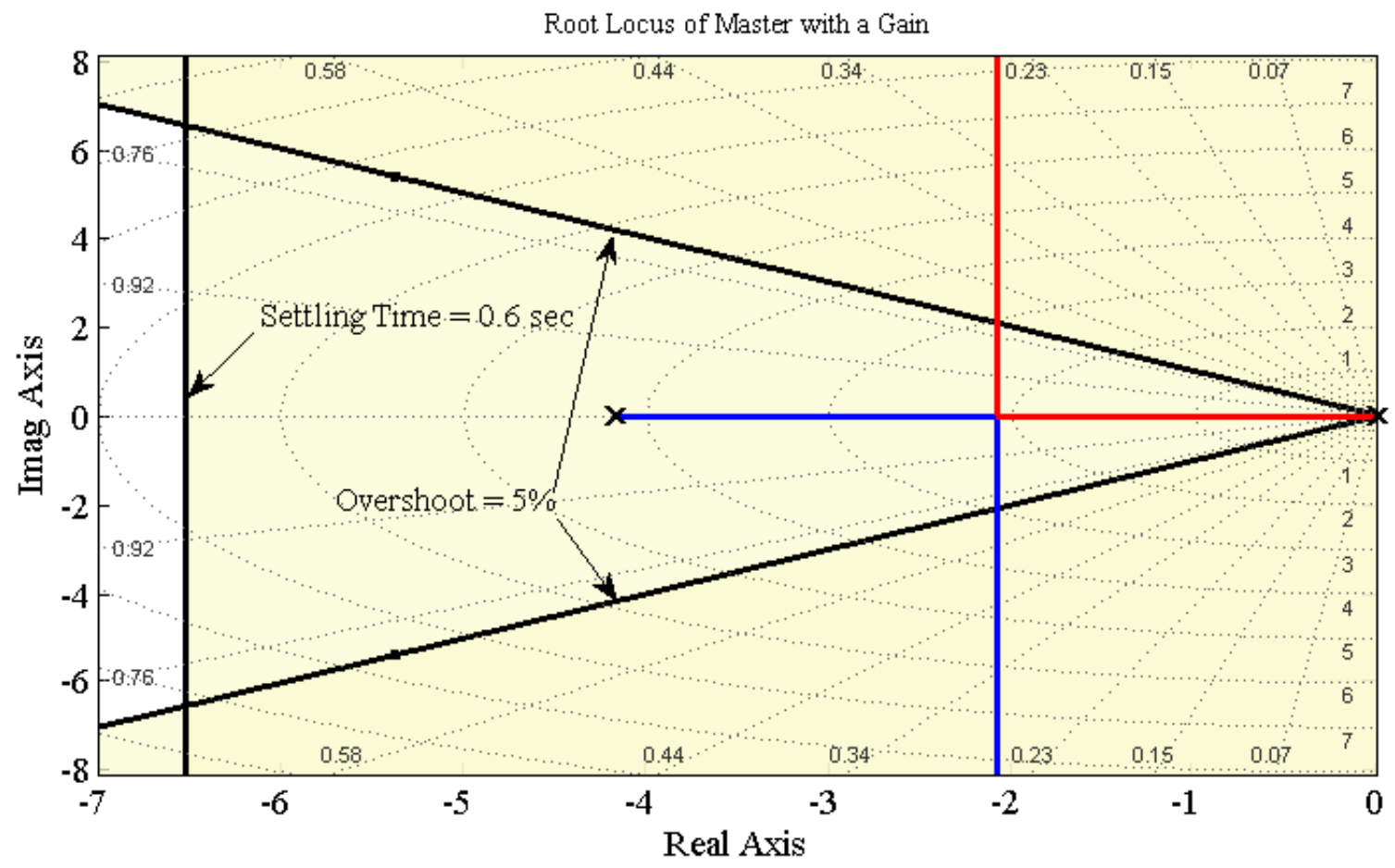

Figure 5. 16. Root locus of the master with a gain controller

where the unshaded area is the acceptable area. As shown in Figure 5. 16, with a gain controller the desired criteria cannot be met since with a gain controller increases overshoot only.

By integrating a PID controller, the path will be shaped such that it goes toward the unshaded area. For this purpose, PID control was treated as a part of the plant transfer function and poles and zeros of the PID were set such that the root locus for $K_{\text {rlocus }}=1$ (root locus gain) satisfies the design criteria. As discussed before, PID controller has two poles and two zeros. One pole is at the origin and the other pole is equal to the value of the filter coefficient $N$. In order to tune the controller such that the root locus moves toward the unshaded area, zeros of the PID controller were set to -7.48 and -1 , poles were set to 50 and 0 , and $K_{P I D}=18$. 


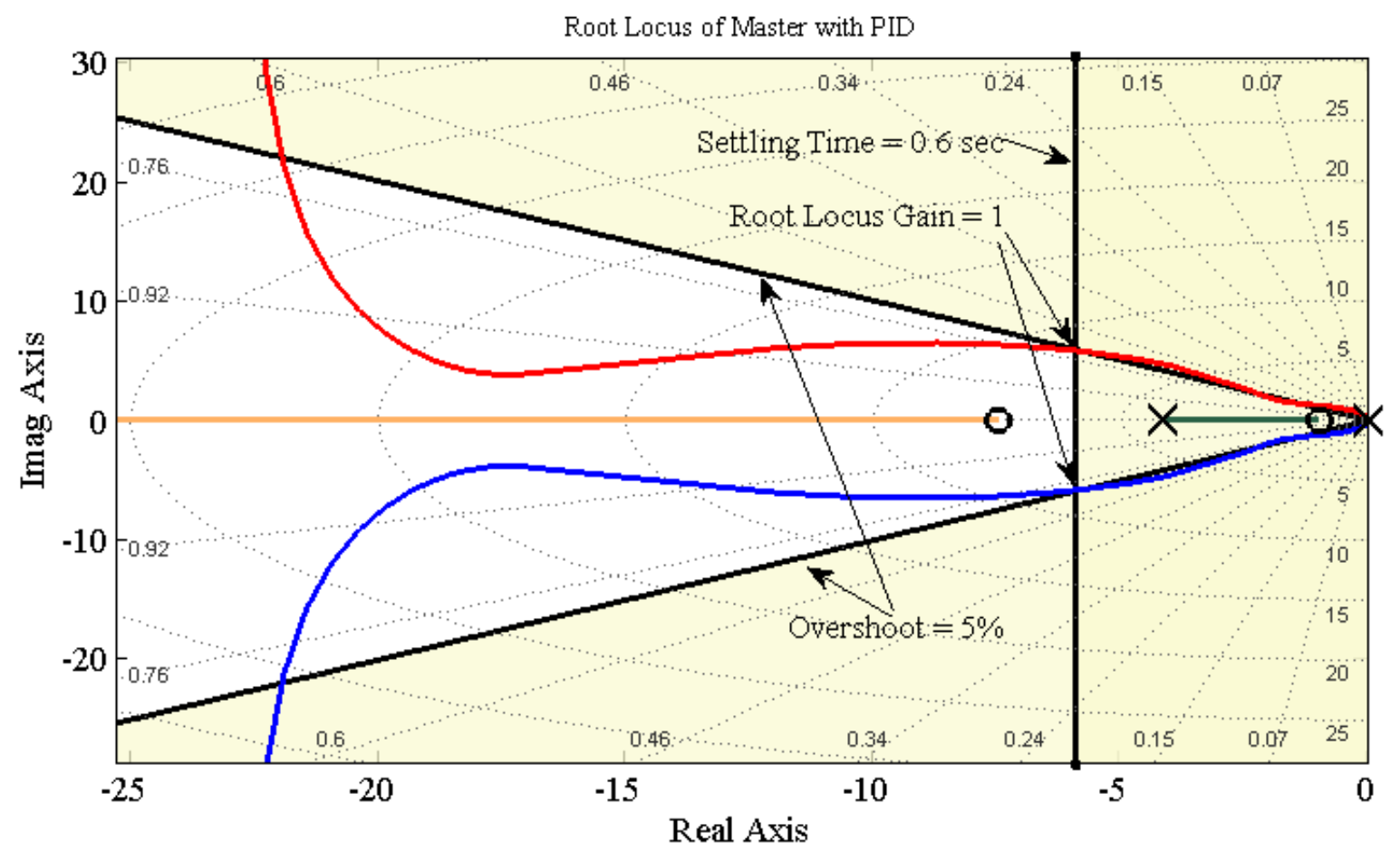

Figure 5. 17. Root Locus of master with PID controller

Using (5. 18) and substituting it in (5.17), the following gains were obtained.

Table 5. 3. Gains of the PID controller of the master

\begin{tabular}{cccc}
$K_{P}$ & $K_{I}$ & $K_{D}$ & $N$ \\
\hline 3 & 2.7 & 0.3 & 50
\end{tabular}

\section{Slave Tuning}

The desired requirements for slave controller is $10 \%$ overshoot and 0.15 seconds settling time. The settling time was very small in order to compensate for the delay between the slave and master. Overshoot was set high in order to have a quick settling time, which means that the controller was high-gain type. The root locus of the slave actuation is: 


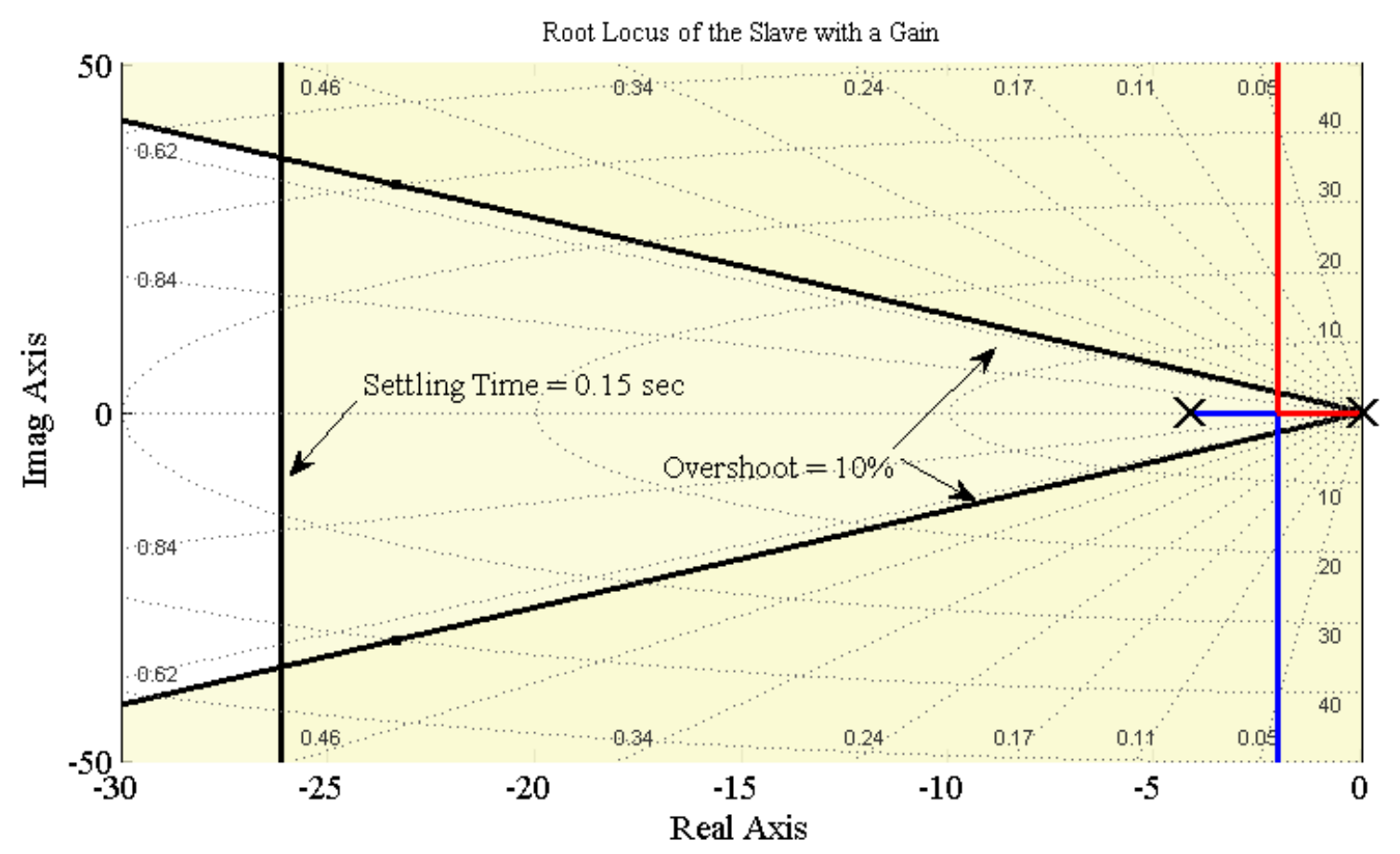

Figure 5. 18. Root Locus of the slave with a gain

Since the closed loop system for the slave actuator cannot be controlled using simple proportional control, a PID controller was used. Thus, the root locus of the slave is.

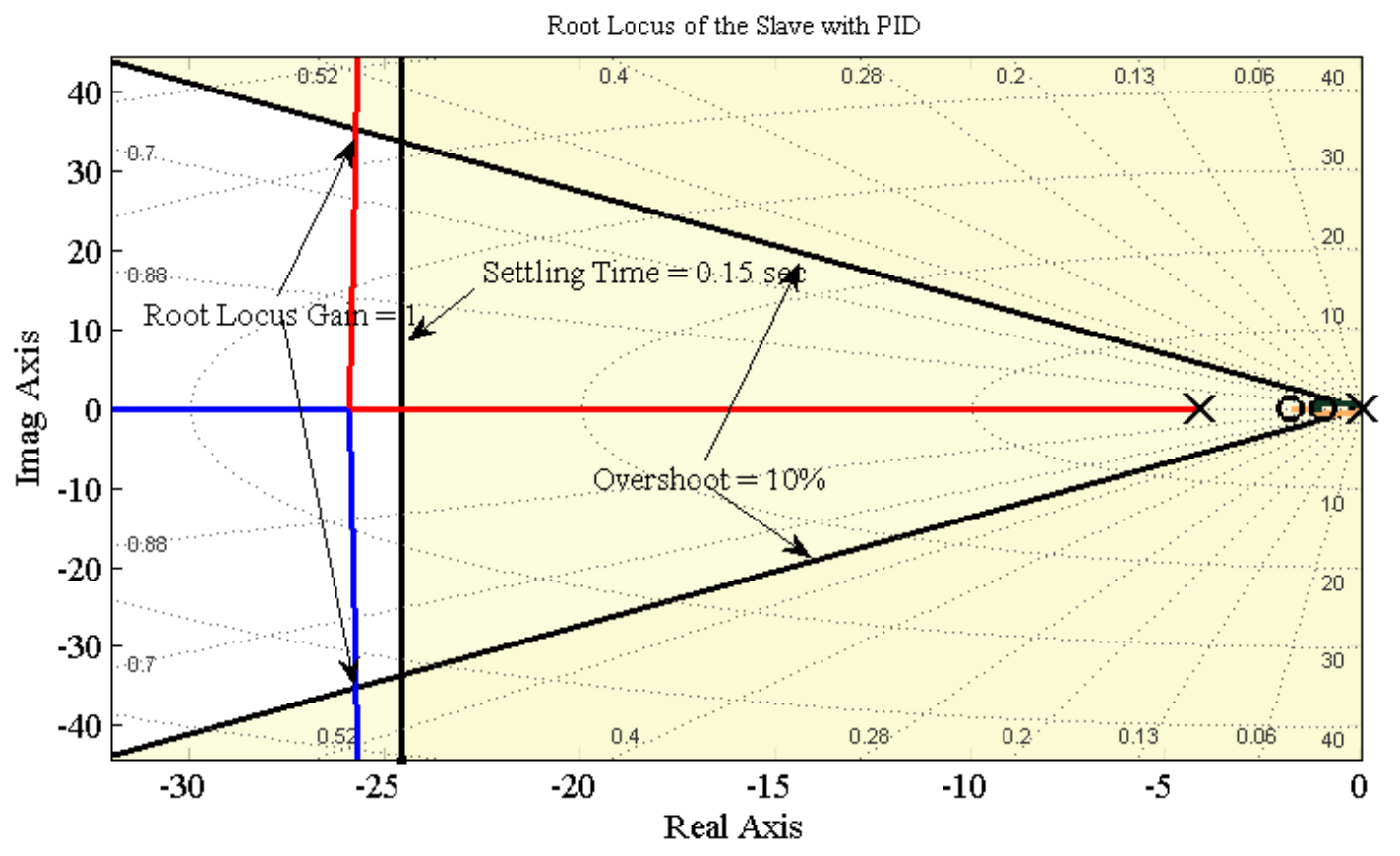

Figure 5. 19. Root locus of the slave with PID 
One pole was at the origin and the other pole was equal to the value of the filter coefficient $N$. The zeros of the PID controller were set to -1.84 and -1.02 , poles were set to 0 and 50 , and $K_{P I D}=530$. Using (5.18) and substituting it in (5.17), the following gains were obtained.

Table 5. 4. Gains of the PID controller of the slave

\begin{tabular}{llll}
$K_{P}$ & $K_{I}$ & $K_{D}$ & $N$ \\
\hline 30 & 20 & 10 & 50
\end{tabular}

\section{Disturbance Rejection}

Master and slave outputs as a function of input disturbances were derived in (5.1) and (5. 7). The two equations are shown below:

$$
\begin{gathered}
Y_{M}=\frac{G_{M} G_{T A} G_{A}}{1+C_{M} G_{M} G_{T A} G_{A}}\left(C_{M} R_{M}-K C_{M} D_{e}+C_{M} D_{A}+D_{M}\right), \\
Y_{S}=\frac{G_{S}}{1+C_{S} G_{S}}\left(C_{S} R_{S}-D_{S}\right),
\end{gathered}
$$

Two types of disturbances were applied to the system as shown in (5. 19) and (5. 20): disturbance applied by the slave to the desired trajectory of the master and disturbances applied to master and slave caused by an external torque, unexpected variation of voltage or current, or current limited by the power supply. In the following each one of these disturbances and their effects on the response of the system were discussed.

As shown in (5.19), three disturbances were applied to the master actuator. Two of them were applied by the slave which are $D_{e}$ (tracking error of the slave) and $D_{A}$, (angular error due to fast or slow response of the slave). The purpose of the tracking error disturbance was to synchronize the slave with the master such that the master had feedback from the slave, if there was high disturbance on slave. This disturbance could cause slow response of the slave and consequently 
high tracking error of the slave. The value of the tracking error disturbance was always negative since the string actuation must be in tension to work. Thus, if compressed, it did not apply any disturbance on the actuator. Since the disturbance was applied to the desired trajectory of the master, the only way to reduce its effect on the master was bounding it and keeping the value small by selecting low $K$ gains. However, low gains reduce the effect of the synchronization, which eventually results in potential conflicts between master and slave and high angular error of the system. The value of $K$ needs special consideration too. If the value of $K$ is too big, the tracking error of the slave will overweight the angular feedback, which causes instability. $K$ must be tuned manually and case by case depending on the predicted disturbances on master and slave. The value of $K$ for this experiment were set to 5 .

The angular error, $D_{A}$, which is the disturbance showed up in the output of the angular encoder is caused by the inconsistency in the behavior of the strings. These inconsistencies could be caused by the strings wearing out or permanent elongation of the strings during time. Since the position of the slave was calculated using the pre-recorded data from the string, any deviation from the prerecorded model can cause this disturbance. The value of this disturbance was very small and negligible and it only became tangible if the strings were damaged or they have been exposed to high forces resulting in permanent deformation of strings.

The third and last disturbance on the master actuator $\left(D_{M}\right)$, which is similar the same type applied to slave too $\left(D_{S}\right)$, is because of external force. This force could be applied by the weight of the human user or friction between mechanical components of the system. Disturbance rejection for this type of disturbance is very important and the controller should be able to overcome that quickly. Otherwise external forces decrease the performance by slowing down one of the actuators whose effect appears as other types of disturbances applied to the cross-coupled actuator, which 
consequently results in poor performance of the whole system. Considering the importance of this issue, the gains of the controller discussed before were chosen such that they reject disturbance rapidly. The response of the master actuator to a unit disturbance is shown below. The unit disturbance is in volts and the output is the position velocity of the actuator in a closed loop system.

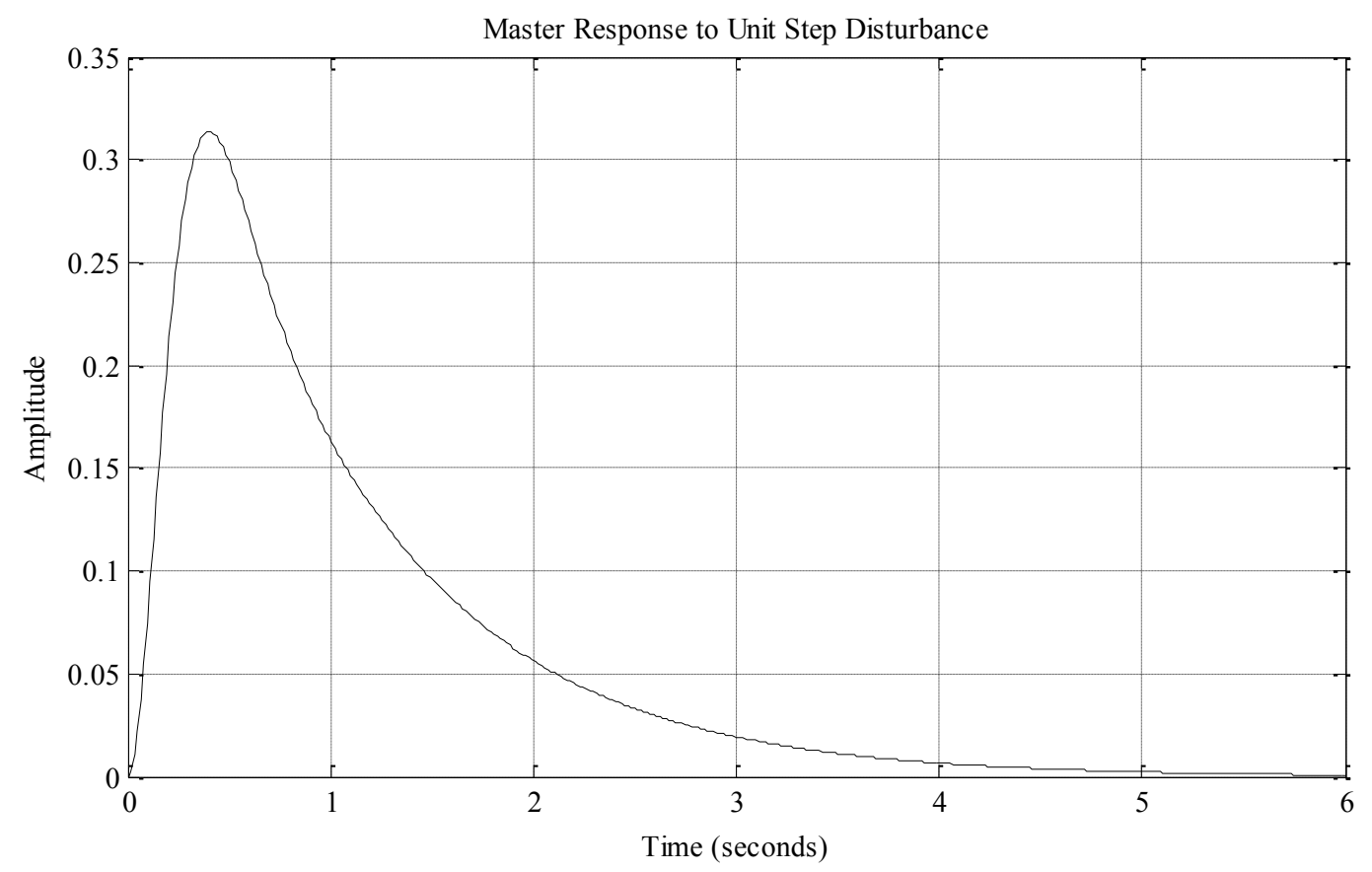

Figure 5. 20. Response of master to unit disturbance

As shown, disturbance was damped rapidly in 2 seconds and the steady-state error was zero. The response of the slave actuator is shown in Figure 5. 21. The unit step disturbance was damped in 4 seconds and the steady state error was zero. 


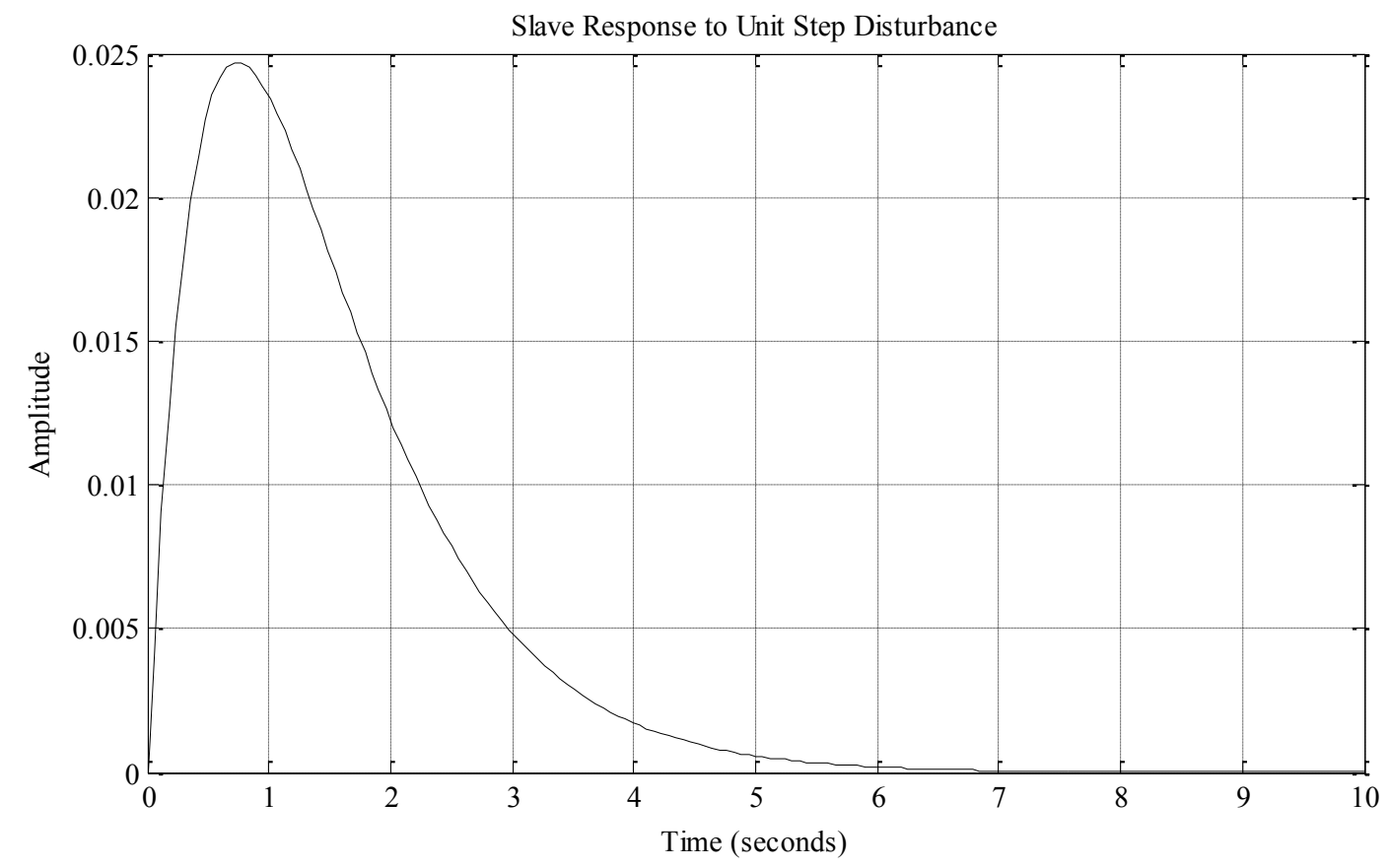

Figure 5. 21. Response of slave to unit disturbance

\section{Tracking Performance}

Tracking performance of the master and slave, or in other words synchronization, is a very crucial issue. If the delay between the master and slave is too high, the slave cannot track the desired trajectory, which results in poor performance. Thus, to evaluate the performance of the synchronization of the master and slave, a trajectory was generated, which the master should follow. The slave, however, should follow the trajectory of the master. The trajectory of the master was acquired through the encoder mounted on the master. In this phase, it was assumed that there was no disturbance on the master or slave. The architecture of the synchronization is shown in Figure 5. 22. 


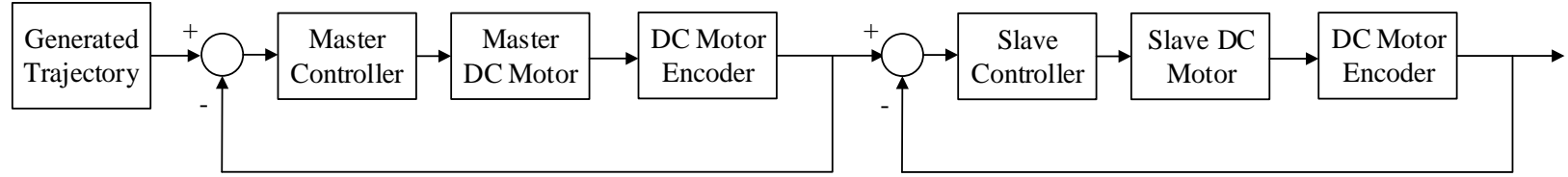

Figure 5. 22. The architecture of the synchronization control

To evaluate system performance, an increasing sinusoidal signal with an initial value was generated whose frequency is low enough to be tracked by actuator. This sinusoidal trajectory was selected since it was complex enough to capture almost all characteristics of a system and show if they track each other well or not. Performance of the controller is shown in Figure 5. 23 without using any anti-windup method.

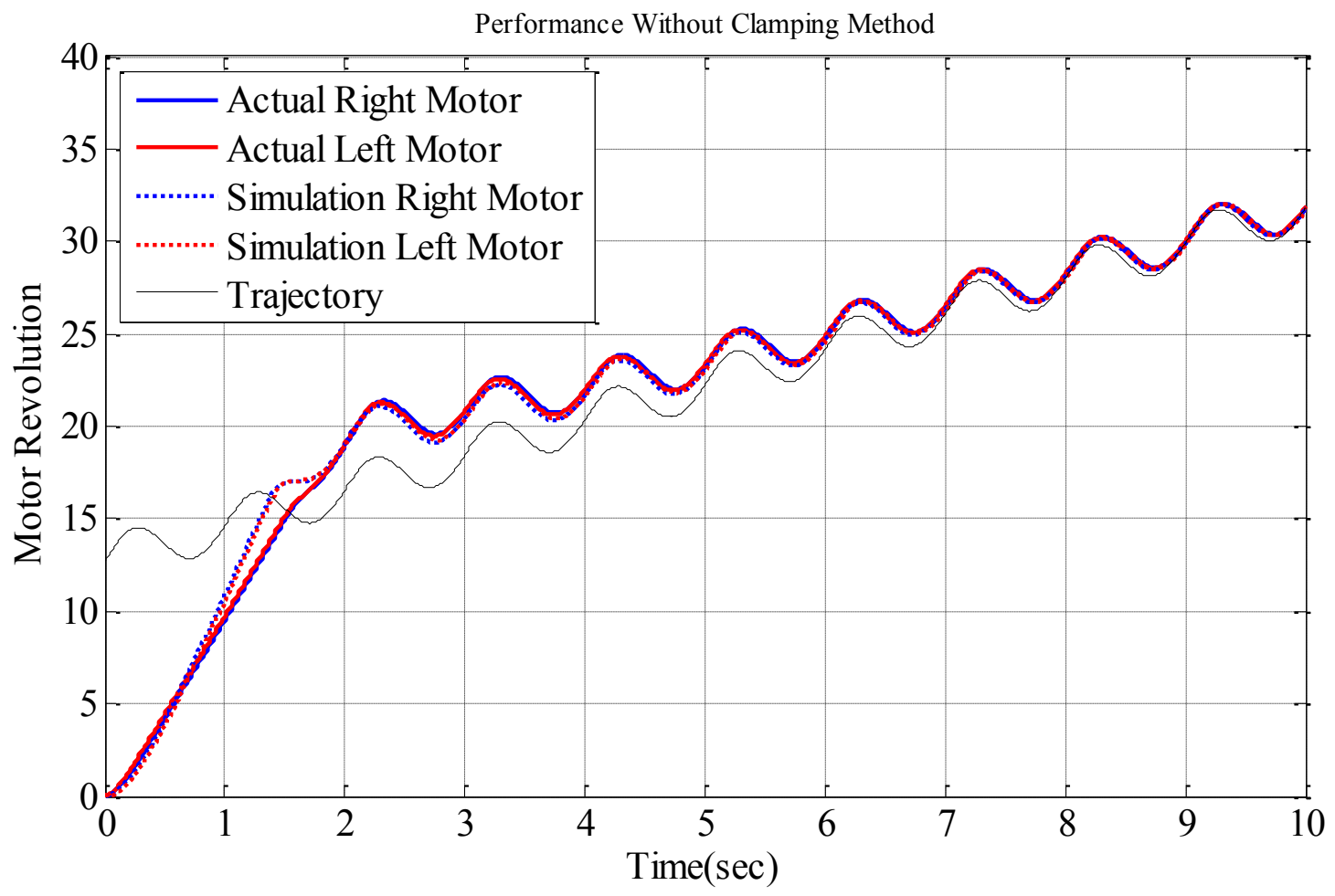

Figure 5. 23. Trajectory performance of the master-slave synchronization control without using anti-windup method 
As shown, simulation and the actual data match very well; however, the performance is poor in the transient response because of the built-up error. The maximum of 35 revolutions is selected according to the range of motion, which will be used. Using the clamping method, the transient response will be improved significantly.

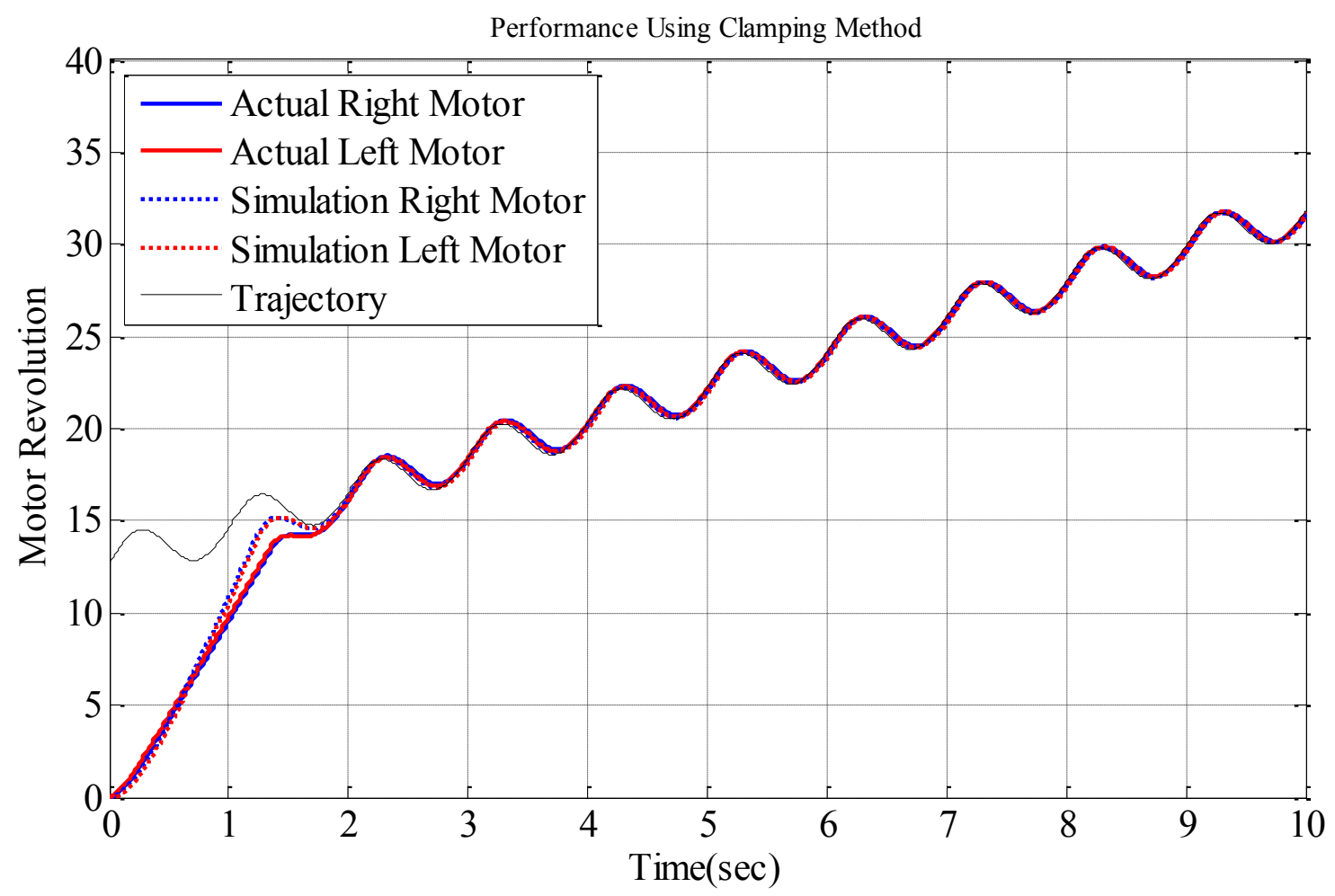

Figure 5. 24. Trajectory performance of the master-slave synchronization control using anti-windup method

The PID gains for both tests are mentioned in Table 5. 5.

Table 5. 5. Gains used for the synchronization control

\begin{tabular}{llll}
$K_{P}$ & $K_{I}$ & $K_{D}$ & $N$ \\
\hline 7.75 & 2.53 & 1.85 & 86.56
\end{tabular}




\subsection{ADL Trajectory, Control Performance, and Experimental Procedures}

To ensure that the assistive robotic system can perform ordinary ADL, it was desired to develop a compensator to drive the mechanism to follow the trajectories of specific human locomotion. In 2012, Cheng et al. [221] investigated the motion of the shoulder angle and elbow angle while performing an ADL, which was picking up a glass of water to drink and putting it back on the table. The test was performed for three independent subjects (healthy male adults). Figure 5. 25 and Figure 5. 26 illustrate the motion and angular movement of the elbow angles from the test. It is clear that the angular movements of elbow were similar for this specific motion.

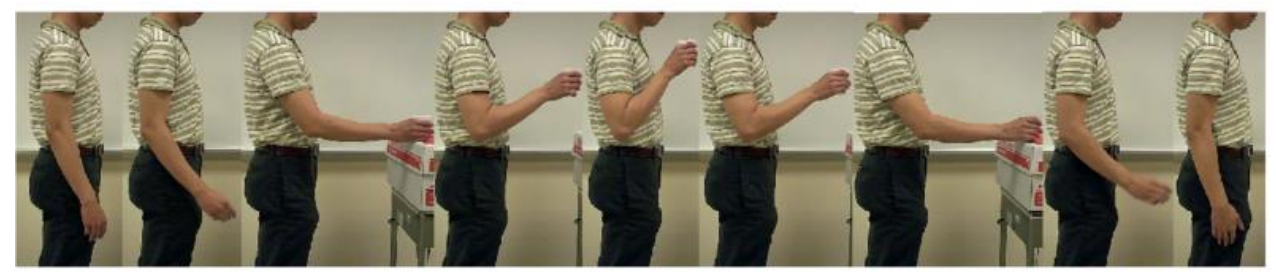

Figure 5. 25. Upper extremities motion at the test to drink water [221]

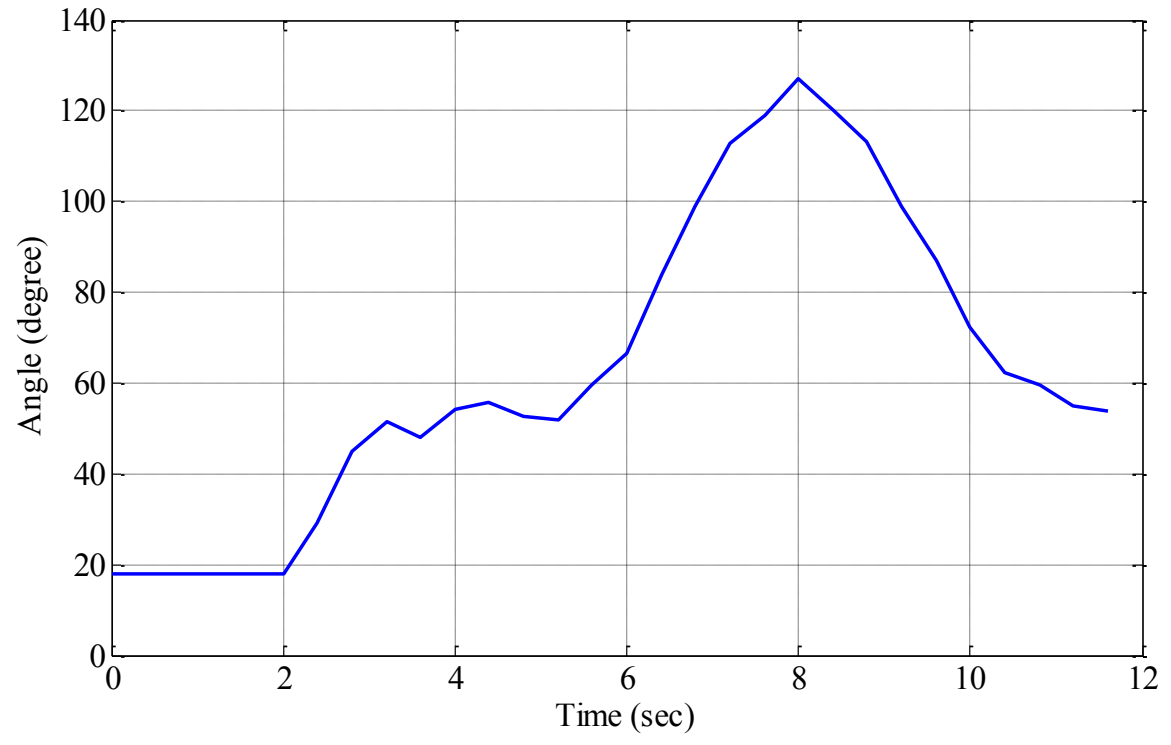

Figure 5. 26. Elbow joint angle while performing targeted ADL 
The test has three phases: pre-twisting the strings, ADL operation, and going back to untwisted position. It should be noted that the actuation goes back to the untwisted mode at the end of each test in order to be ready for the next test.

Applying the PID control to the system the following results were achieved.

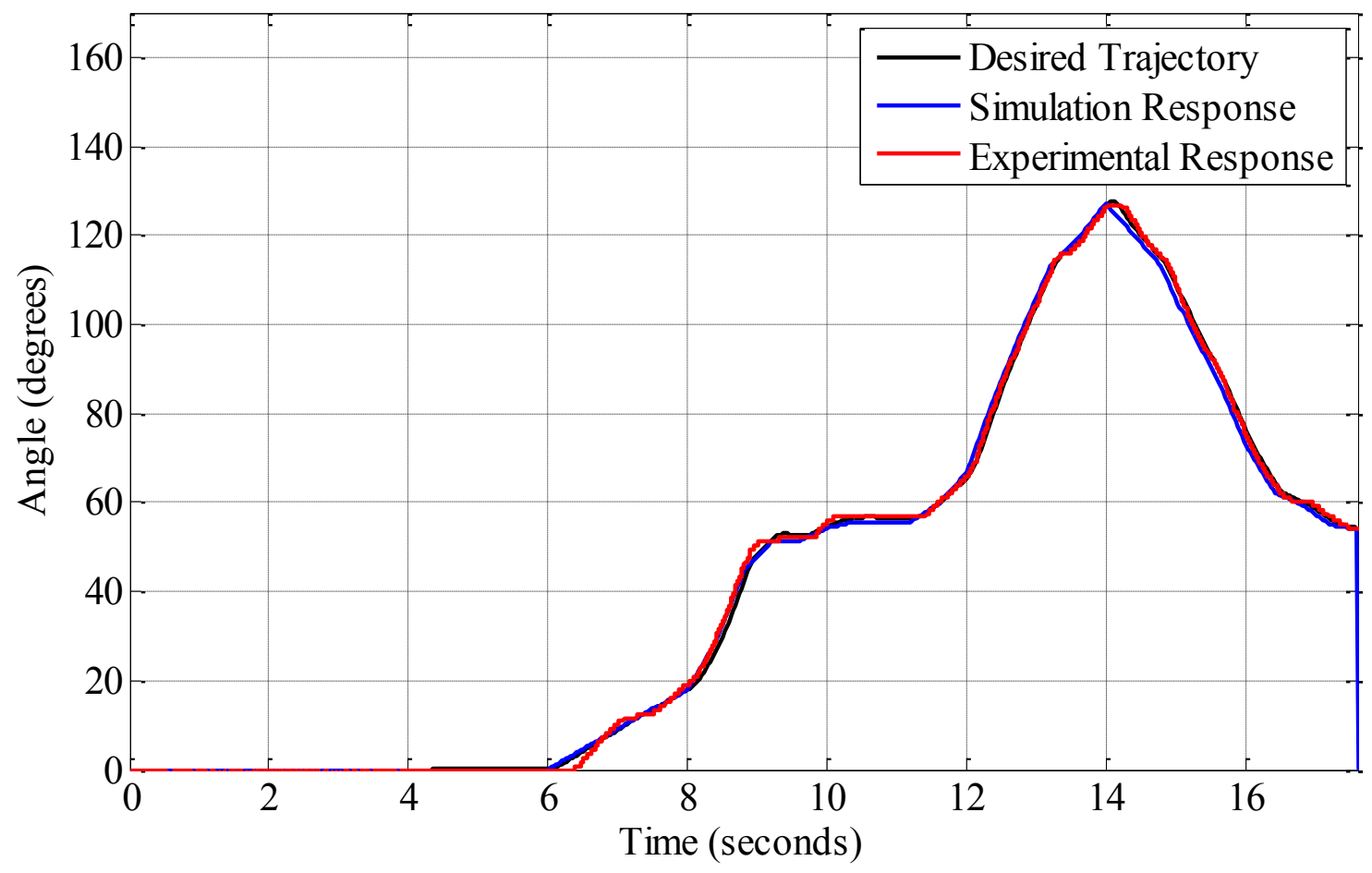

Figure 5. 27. Angle trajectory of the simulation and experiment

The tracking error between the desired trajectory and the results from simulation and experiment are shown below. There was a maximum of 4 degrees tracking error by the elbow angular encoder. 


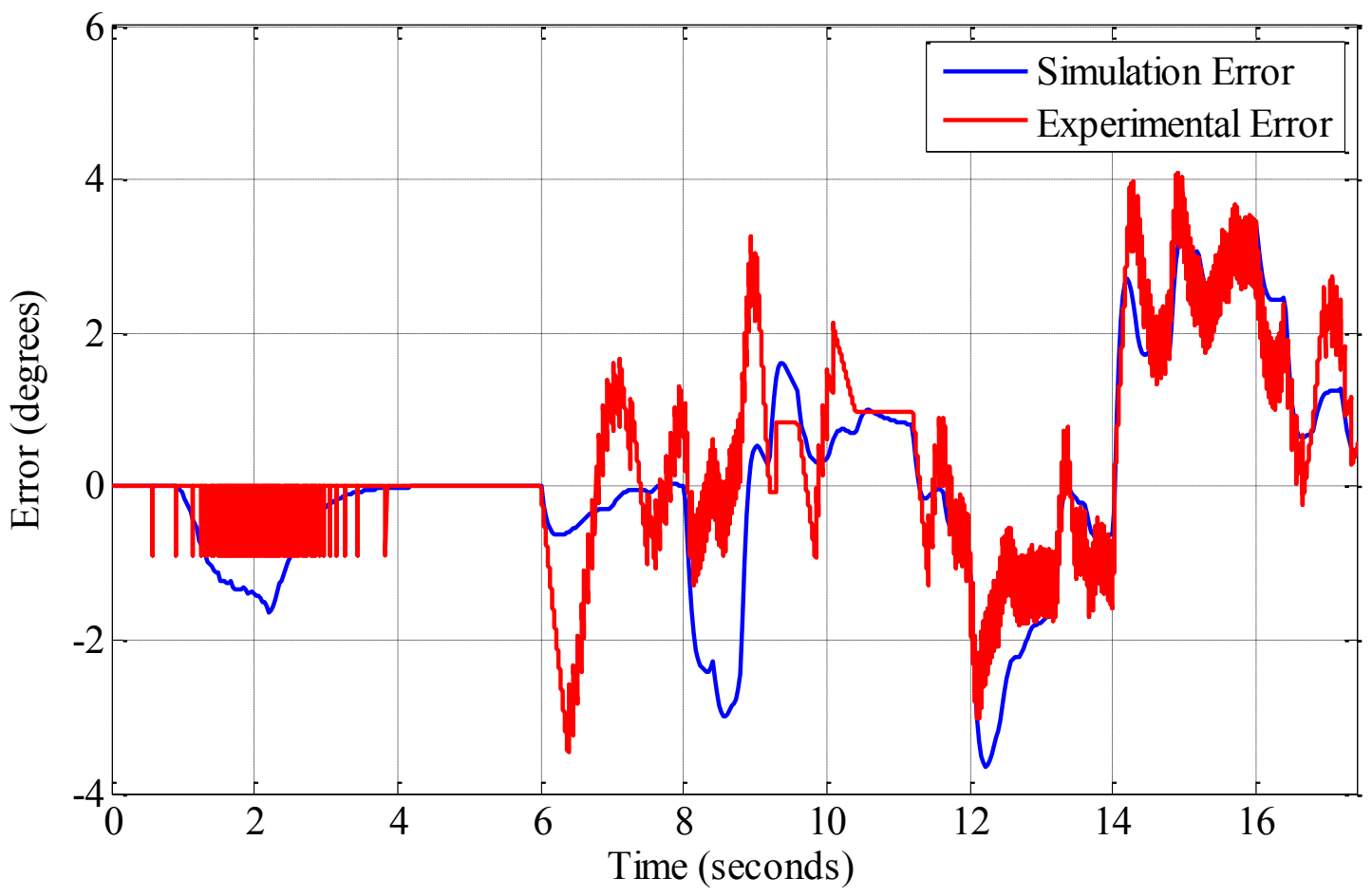

Figure 5. 28. Tracking error of the simulation and experiment

Even though the slave was tracking the contraction of the master, there might be a contraction conflict between master and slave, which was addressed before. In other words, the slave was not tracking the master very well. The error displacement of the pulley due to master/slave tracking errors were captured and results are shown below. 

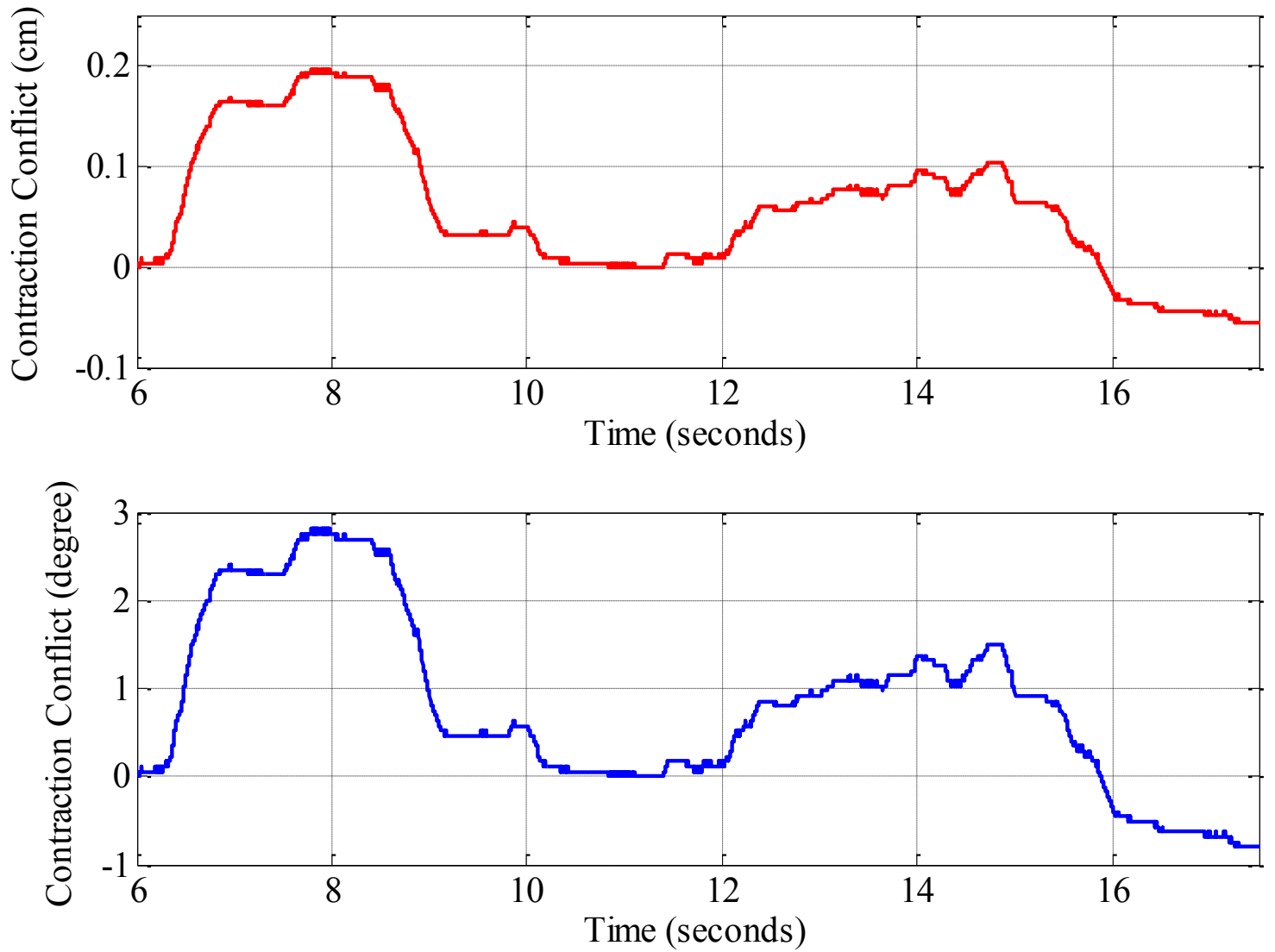

Figure 5. 29. Contraction error

As shown, the maximum contraction error recorded was $2 \mathrm{~mm}$. and the corresponding angular error by the elbow angle was 3 degrees.

Considering tracking error and angular error, the total possible error in degrees was 7.

\subsection{Torque Control and Estimation}

The proposed control method in previous section were based on position control with the assumption that enough current was available. However, if there is not enough torque available to perform the task, position control is meaningless. In other words, the required torque to maintain 
position is correlated with the generated toque by the actuator. On the other hand, the generated torque by the actuator is correlated with the available power supply current. In other words, if the maximum available current generated by the power supply is not equal or more than the required current corresponding to the required torque to perform an ADL task, position control is meaningless. Thus, in order to select a proper power supply, it is essential to compute the maximum required current to perform an ADL. To compute the required current while performing an ADL, a flow chart was presented as following:

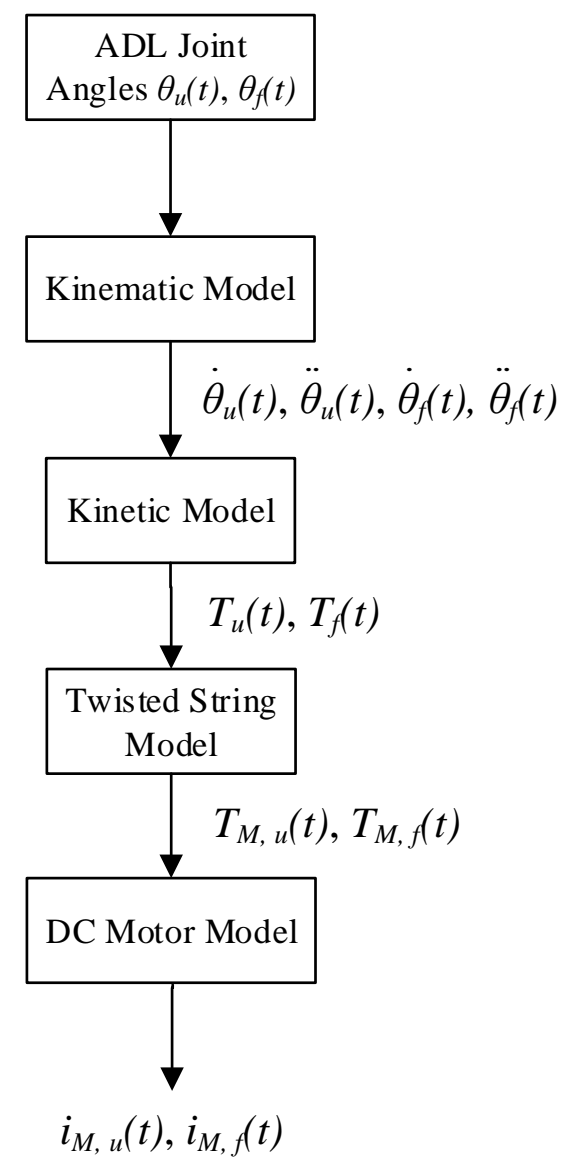

Figure 5. 30. Flowchart of using appendix A and B to compute the required current

where $\theta_{u}(t)$ and $\theta_{f}(t)$ are the desired angles of upper arm and forearm, $T_{u}(t)$ and $T_{f}(t)$ are the required torque at the place of upper arm and forearm joints, $T_{M, u}(t)$ and $T_{M, f}(t)$ are the required torque by actuators; and $i_{M, u}(t)$ and $i_{M, f}(t)$ are required current to drive the actuators. 
To compute the required torque, two separate kinetic and kinematic models were derived in appendix A and B. Considering the desired position of upper extremities while performing an ADL (as a function of time), and applying the kinematic model in appendix B, acceleration and velocity of the model can be obtained. Knowing the physical properties of the system such as mass and moment of inertia; and applying the resultant position, velocity, and acceleration (from kinematic model) to the kinetic model, the required torque at each joint can be calculated. Knowing the position and orientation of the twisted string actuation, the required actuator torque can be computed. Ultimately, considering the current constant of the actuator, $K_{i}$, required current can be calculated. 


\section{Chapter 6: Conclusions and Future}

\section{Works}

\subsection{Conclusion}

In this work a wearable, light assistive robot was designed, which was able to cover a wide range of motion of the upper extremities while doing ADLs. Actuation was provided by a novel and compact actuation system called the twisted string method. Ultimately a controller was designed and its performance was evaluated based on the tracking error of the ADL trajectory.

There were restrictions and novelties in this work, which should be discussed in detail. These novelties and restrictions were categorized in three categories: mechanical design, actuation, and control.

\section{Mechanical Design}


The mechanical design included the structure of the exoskeleton robot, which should be comfortable and adjustable for a range of patients. Mechanical design had three major components: the backpack platform, the shoulder complex, and the elbow.

The backpack platform had two pieces (Figure 3. 19), which were adjustable for the target range of patients. One of the major challenges for any exoskeleton design is the shoulder joint. The shoulder complex had 5 joints, while the first two prismatic joints were fixed on the backpack platform and they were just for the purpose of adjustability. Unlike the first two links, the second two links (revolute joints) were custom-designed (Figure 3. 23). In other words, they were made according to each patient's shoulder size. It should be noted that these two links could be designed to be adjustable; however, it could make the final design more complex, bulky, and expensive.

Any proposed configuration for the shoulder joint is debatable from the perspective of the sequence and number of links, which affects the complexity, comfort, and range of motion that an exoskeleton can provide. Thus it is a trade-off between cost, comfort, and targeted range of motion. In this work, the shoulder complex was modeled with three consecutive joints. The motion of the scapula was neglected since the robot was designed for ADLs in which scapular motion was in the stable region. The exoskeleton cannot provide shoulder abduction more than $60^{\circ}\left(\psi_{1}=60^{\circ}\right.$ in Figure 3.26) and once it reaches the maximum angle, a mechanical stop prevents the shoulder link from further abduction. To provide a bigger range of motion, the number and sequence of joints must be changed.

\section{Actuation System}

The twisted string actuation method was used to actuate the robot. It is a compact and affordable actuation, with high power-to-weight ratio. Moreover, it does not use any intermediate device such as a gear box, which reduces the size and weight of the actuation system. The length of the 
actuation, however was one of the drawbacks of this method. As a rule of thumb for each $10 \mathrm{~cm}$ of actuation contraction, $50 \mathrm{~cm}$ of twisted string was needed, which could undermine the compactness feature of the actuation. Moreover, the twisted string was susceptible to load change and depending on how many strings were used, actuation could get elongated. Tactical cables made of carbon composite materials were chosen as the best "strings" for the actuator. Different configurations were tested, but the chosen configuration was with 6 twisting strings and a neutral string. When tested under $5 \mathrm{~N}$ to $35 \mathrm{~N}$ loads, the maximum variation in contraction under this load change was $0.9 \mathrm{~cm}$. considering the target of $20 \%$ assistance, the maximum static load is expected to lie between 19 to $41 \mathrm{~N}$ for the elbow and 30 to $64 \mathrm{~N}$ for the shoulder joint. These values are almost double the test case loads. Thus, it is expected that bigger contraction variations will be observed if bigger loads are applied. This means that the actuation should be tested under all these loads and its behavior should be fully captured in order to be used for the current control system unless a more robust controller is used. Thus, it is suggested to use this actuation for light applications such as finger exoskeleton or elbow actuation. This actuation is not suitable for providing high axial force for full assistance, due to the variation of contraction under widely varying loads.

Nonlinearity was the other issue with this type of actuation, which could make the control design more difficult. To select a more linear range of contraction/release the total length of the actuation must be increased, which may exceed the physical dimensions of the desired actuation. Moreover, wear out signs were observed after 200 cycles, which may be the result of the interaction between aluminum cable holders and strings. Further experimentation is required to characterize the durability of the twisted string actuator. 


\section{Control System}

A master-slave control was used for this system. Easy implementation and flexibility to be merged with other control methods are major features of this control strategy. One of the intrinsic drawbacks of the master-slave control is delay of the slave with respect to master. This issue, which was observed in this work too, could be mitigated by a feed forward signal from the desired trajectory of the master to the desired trajectory of the slave. Using this method, however, may result in the slave performing faster than the master if a high disturbance load was applied to the master. Moreover, the master is synchronized to the slave by the tracking error feedback with a gain. This gain must be tuned manually and based on the potential range of disturbance on the slave it can be big or small.

\subsection{Recommendations}

It is recommended to use a high-torque actuators such as a pneumatic actuator at least for the shoulder joint. All control tests in this work were done under limited/bounded disturbance. Thus, higher disturbances should be applied in order to ensure stability of the controller.

It is also recommended to use a more stable controller, which does not inherit the slave delay. For this purpose a cross coupled controller is suggested. Force feedback has been used in almost all previous works using twisted string actuation. Force can be sensed using force gauges or a small spring attached to the actuation. Both of these two way can be used to send a feedback from slave if it is following master or not. In this work all these sensors removed in order to keep the device affordable and compact. Thus, it is recommended to use force feedback for the control unit. 


\subsection{Future Work}

As for the controller I will extend my work and use a cross-coupling controller to improve the controller performance. An adaptive scheme may be used to improve the adaptability of the controller against weight variation. Moreover, I will integrate electromyography (EMG) sensors as force feedback from the human upper extremities to the controller such that the desired position or torque can be calculated using these signals. A kinematic model was derived in appendix B, using which position, velocity, and acceleration of any ADL can be determined. Using this information in the kinetic model derived in appendix A, the required torque for the joints could be determined. On the other hand, if the same position, velocity, and acceleration of joints were sensed by sensors, using this model the required torque could be determined online. This information can be used for calculated torque control method. From the mechanical perspective I would like to work on a more sophisticated design for the shoulder joint, which includes the scapular motion and does not undermine the affordability and simplicity of design. 


\section{References}

[1] Wikipedia, "Stroke," Wikipedia, [Online]. Available: http://en.wikipedia.org/wiki/Stroke. [Accessed 189 2013].

[2] G. A. M. Donnan, M. M. Fisher and M. D. S. M. M. Macleod, "Stroke," The Lancet, vol. 373, no. 9674, p. 1612-1623, 2-8 May 2009.

[3] C. J. D. Murray and A. D. P. Lopez, "Mortality by cause for eight regions of the world: Global Burden of Disease Study," The Lancet, vol. 349, no. 9061, p. 12691276, 3 May 1997.

[4] L.-J. D. A. R. B. T. C. M. D. S. D. S. G. F. T. F. E. F. K. G. C. G. A. G. K. H. N. H. S. H. P. H. V. K. B. K. S. L. D. L. L. M. A. e. WRITING GROUP MEMBERS, "American Heart Association Statistics Committee and Stroke Statistics Subcommittee: Heart disease and stroke statistics-2010 update: a report from the American Heart Association," American Heart Association , 2010.

[5] V. L. Feigin, "Stroke epidemiology in the developing world," The Lancet, vol. 365 , no. 9478 , p. 2160-2161, 25 June-1 July.

[6] "The world health report 2004: changing history; Annex Table 2: Deaths by cause, sex and mortality stratum in WHO regions, estimates for 2002," World Health Organization, Geneva, 2004.

[7] W. Rosamond, K. Flegal, G. Friday, K. Furie, A. Go, K. Greenlund, N. Haase, M. Ho, V. Howard, B. Kissela, S. Kittner, D. M. M. Lloyd-Jones, J. Meigs, C. Moy, G. Nichol, C. O'Donnell, V. Roger, J. Rumsfeld, P. Sorlie, J. Steinberger, T. Thom, S. Wasserthiel-Smoller and Y. Hong, "Heart disease and stroke statistics--2007 update: a report from the American Heart Association Statistics Committee and 
Stroke Statistics Subcommittee," American Heart Association Statistics Committee and Stroke Statistics Subcommittee., vol. 115, no. 5, pp. 69-171, Feb 62007 .

[8] E. Hanne, J. Holmen, B. Indredavik and A. Terent, "Epidemiology of stroke in Innherred, Norway, 1994 to 1996 incidence and 30-day case-fatality rate.," Stroke, vol. 28, no. 11, pp. 2180-2184, 1997, Norway.

[9] R. C. Senelick, K. Dougherty and P. W. Rossi, Living with stroke: A guide for families, Chicago: NTC/Contemporary Pub., 1999.

[10] S. (. O. o. S. a. H. R. National Institute of Neurological Disorders, "Chronic pain: Hope through research," The Office, 1998.

[11] P. W. Duncan, R. Zorowitz, B. Bates, J. Y. Choi, J. J. Glasberg, G. D. Graham, R. C. Katz, K. Lamberty and D. Reker, "Management of adult stroke rehabilitation care a clinical practice guideline," Stroke, Am Heart Assoc, vol. 36, no. 9, pp. e100e143, 2005.

[12] Wikipedia, "Stroke recovery," Wikipedia, [Online]. Available: http://en.wikipedia.org/wiki/Stroke_recovery. [Accessed 189 2013].

[13] S. Licht, Stroke and its rehabilitation, Williams \& Wilkins, 1975.

[14] T. E. Twitchell, "The restoration of motor function following hemiplegia in man," Brain, Oxford Univ Press, vol. 74, no. 4, pp. 443-480, 1951.

[15] R. J. Nudo, B. M. Wise, F. SiFuentes and G. W. Milliken, "Neural substrates for the effects of rehabilitative training on motor recovery after ischemic infarct," Science, American Association for the Advancement of Science, vol. 272, no. 5269, pp. 1791-1794, 1996.

[16] R. J. Nudo, E. J. Plautz and S. B. Frost, "Role of adaptive plasticity in recovery of function after damage to motor cortex," Muscle Nerve, Wiley Online Library, vol. 24, no. 8, pp. 1000-1019, 2001. 

G. Abrams, "Effectiveness of sensory and motor rehabilitation of the upper limb following the principles of neuroplasticity: patients stable poststroke," Neurorehabil.Neural Repair, SAGE Publications, vol. 17, no. 3, pp. 176-191, 2003.

[18] G. L. K. R. A. P. J. G. BROEKS, "The long-term outcome of arm function after stroke: results of a follow-up study," Disability \& Rehabilitation, Informa UK Ltd UK, vol. 21, no. 8, pp. 357-364, 1999.

[19] M. Kassler, "Robotics for health care: a review of the literature," ROBOTICACAMBRIDGE, Cambridge Univ Press, vol. 11, pp. 495-495, 1993.

[20] M. Hillman, "Introduction to the special issue on rehabilitation robotics," Robotica, Cambridge Univ Press, vol. 16, no. 5, pp. 485-485, 1998.

[21] S. Demain, R. Wiles, L. Roberts and K. McPherson, "Recovery plateau following stroke: Fact or fiction?," Disability \& Rehabilitation, Informa UK Ltd UK, vol. 28, no. 13-14, pp. 815-821, 2006.

A. A. Timmermans, H. A. Seelen, R. P. Geers, P. K. Saini, S. Winter, J. te Vrugt and H. Kingma, "Sensor-based arm Skill Training in Chronic Stroke patients: results on treatment outcome, patient motivation, and system usability," Neural Systems and Rehabilitation Engineering, IEEE Transactions , IEEE, vol. 18, no. 3, pp. 284-292, 2010.

B. R. Brewer, S. K. McDowell and L. C. Worthen-Chaudhari, "Poststroke upper extremity rehabilitation: a review of robotic systems and clinical results," Topics in stroke rehabilitation, Thomas Land, vol. 14, no. 6, pp. 22-44, 2007.

R. Gopura, K. Kiguchi and D. Bandara, "A brief review on upper extremity robotic exoskeleton systems," in Industrial and Information Systems (ICIIS), 2011 6th IEEE International Conference on, 2011, 346-351, 2011. 

Science, vol. 187, no. 5, p. 70-73, 1965.

[26] V. L. NICKEL, A. KARCHAKJR and J. R. ALLEN, "Electrically Powered Orthotic Systems," J Bone Joint Surg Am, vol. 51, no. 2, pp. 343-351, 1969 Mar 01.

G. SCHMEISSER and W. SEAMONE, "An Upper Limb Prosthesis-Orthosis Power and Control System with Multi-Level Potential," J Bone Joint Surg Am, vol. 55, no. 7, pp. 1493-1501, 1973 Oct 01.

[28] M. Vukobratović, Legged locomotion robots and anthropomorphic mechanisms : a monograph, Beograd : Mihailo Pupin Institute, 1975.

[29] N. Benjuya and S. B. Kenney, "Hybrid arm orthosis," JPO: Journal of Prosthetics and Orthotics, vol. 2, no. 2, pp. 155-163, 1990.

[30] H. Kazerooni and S. Mahoney, "Dynamics and control of robotic systems worn by humans," in IEEE International Conference on Robotics and Automation Proceedings, Sacramento, 1991.

[31] G. Kwakkel, R. C. Wagenaar, T. W. Koelman, G. J. Lankhorst and J. C. Koetsier, "Effects of intensity of rehabilitation after stroke a research synthesis," Stroke, Am Heart Assoc, vol. 28, no. 8, pp. 1550-1556, 1997.

[32] S. K. Bhogal, R. Teasell and M. Speechley, "Intensity of aphasia therapy, impact on recovery," Stroke, Am Heart Assoc, vol. 34, no. 4, pp. 987-993, 2003.

R. C. Loureiro, W. S. Harwin, K. Nagai and M. Johnson, "Advances in upper limb stroke rehabilitation: a technology push," Med.Biol.Eng.Comput., Springer, vol. 49, no. 10, pp. 1103-1118, 2011.

G. D. Jacob, "Rehabilitation Therapy," [Online]. Available: http://www.holisticonline.com/Remedies/Heart/stroke_conv_rehab.htm. [Accessed 209 2013]. 
A. S. Association, "Constraint-Induced Movement Therapy," American Stroke Association, [Online].

Available:

http://www.strokeassociation.org/STROKEORG/LifeAfterStroke/RegainingIndepen dence/PhysicalChallenges/Constraint-Induced-MovementTherapy_UCM_309798_Article.jsp. [Accessed 209 2013].

[36] H. I. Krebs, M. Ferraro, S. P. Buerger, M. J. Newbery, A. Makiyama, M. Sandmann, D. Lynch, B. T. Volpe and N. Hogan, "Rehabilitation robotics: pilot trial of a spatial extension for MIT-Manus," Journal of NeuroEngineering and Rehabilitation, BioMed Central Ltd, vol. 1, no. 1, p. 5, 2004.

L. Marchal-Crespo and D. J. Reinkensmeyer, "Review of control strategies for robotic movement training after neurologic injury," Journal of neuroengineering and rehabilitation, BioMed Central Ltd, vol. 6, no. 1, p. 20, 2009.

S. Balasubramanian, R. Colombo, I. Sterpi, V. Sanguineti and E. Burdet, "Robotic Assessment of Upper Limb Motor Function After Stroke," American Journal of Physical Medicine \& Rehabilitation, LWW, vol. 91, no. 11, pp. S255-S269, 2012.

[39] S. Barreca, S. L. Wolf, S. Fasoli and R. Bohannon, "Treatment interventions for the paretic upper limb of stroke survivors: a critical review," Neurorehabil.Neural Repair, SAGE Publications, vol. 17, no. 4, pp. 220-226, 2003.

[40] T. Platz, "Evidence-based arm rehabilitation--a systematic review of the literature," Nervenarzt, Germany, vol. 74, no. 10, pp. 841-849, 2003.

[41] J. Mehrholz, T. Platz, J. Kugler and M. Pohl, "Electromechanical and robotassisted arm training for improving arm function and activities of daily living after stroke," Stroke, Am Heart Assoc, vol. 40, no. 5, pp. e392-e393, 2009.

[42] G. B. Prange, M. J. Jannink, C. G. Groothuis-Oudshoorn, H. J. Hermens and M. J. IJzerman, "Systematic review of the effect of robot-aided therapy on recovery of 
the hemiparetic arm after stroke," Journal of rehabilitation research and development, REHIBILITATION RESEARCH \& DEVELOPMENT SERVICE, vol. 43, no. 2, p. 171, 2006.

R. Riener, "Robot-aided rehabilitation of neural function in the upper extremities," in Operative Neuromodulation, Springer, 2007, pp. 465-471.

[44] J. H. Van der Lee, I. A. Snels, H. Beckerman, G. J. Lankhorst, R. C. Wagenaar and L. M. Bouter, "Exercise therapy for arm function in stroke patients: a systematic review of randomized controlled trials," Clin.Rehabil., SAGE Publications, vol. 15, no. 1, pp. 20-31, 2001.

S. Heidi, "Motor rehabilitation using virtual reality," Journal of NeuroEngineering and Rehabilitation (JNER), vol. 1, no. 1, 2004 . L. P. M. R. E. S. H. T. G. T. C. Gresham GE, Post-stroke rehabilitation. Clinical practice guideline, US Department of Health and Human Services, Public Health Service, Agency for Health Care Policy and Research, 1995.

E. Taub and S. Wolf, "Constraint induced movement techniques to facilitate upper extremity use in stroke patients," Topics in stroke rehabilitation, ASPEN PUBLICATION, vol. 3, pp. 38-61, 1997.

[48] A. Sunderland and A. Tuke, "Neuroplasticity, learning and recovery after stroke: a critical evaluation of constraint-induced therapy," Neuropsychological rehabilitation, Taylor \& Francis, vol. 15, no. 2, pp. 81-96, 2005.

[49] S. H. W. R. K. H. Timmermans AA, "Technology-assisted training of arm-hand skills in stroke: concepts on reacquisition of motor control and therapist guidelines for rehabilitation technology design.," $J$ Neuroeng Rehabil., vol. 6, no. 1, p. 1186/1743, 2009 Jan 20. 
B. E. Fisher and K. J. Sullivan, "Activity-dependent factors affecting poststroke functional outcomes," Topics in stroke rehabilitation, Thomas Land, vol. 8, no. 3, pp. 31-44, 2001.

[51] P. P. Maclean N, "A critical review of the concept of patient motivation in the literature on physical rehabilitation.," Soc Sci Med, vol. 50, no. 4, pp. 495-506, 2000 Feb.

N. Maclean, P. Pound, C. Wolfe and A. Rudd, "Qualitative analysis of stroke patients' motivation for rehabilitation," BMJ: British Medical Journal, BMJ Group, vol. 321, no. $1051,2000$.

C. R. Carignan and H. I. Krebs, "Telerehabilitation robotics: bright lights, big future?," Journal of rehabilitation research and development, REHIBILITATION RESEARCH \& DEVELOPMENT SERVICE, vol. 43, no. 5, p. 695, 2006.

M. Mokhtari, "Java therapy: web-based robotic rehabilitation," in Integration of Assistive Technology in the Information Age, IOS Press, January 1, 2001, pp. 66-71.

V. G. Popescu, G. C. Burdea, M. Bouzit and V. R. Hentz, "A virtual-reality-based telerehabilitation system with force feedback," Information Technology in Biomedicine, IEEE Transactions on, IEEE, vol. 4, no. 1, pp. 45-51, 2000.

[56] P. Bach-y-Rita, S. Wood, R. Leder, O. Paredes, D. Bahr, E. W. Bach-y-Rita and N. Murillo, "Computer-assisted motivating rehabilitation (CAMR) for institutional, home, and educational late stroke programs," Topics in Stroke Rehabilitation, Thomas Land, vol. 8, no. 4, pp. 1-10, 2002.

[57] X. Feng, C. Ellsworth, L. Johnson and J. M. Winters, "UniTherapy: software design and hardware tools of teletherapy," in RESNA 27th International Annual Conference 2004: Technology \& Disability: Research, Design, Practice, \& Policy, , Orlando, FL, 2004. 
J. Johnson Michelle, F. Xin and M. Johnson Laura, "Potential of a suite of robot/computer-assisted motivating systems for personalized, home-based, stroke rehabilitation," J Neuroengineering Rehabil., vol. 4, no. 6, 2007.

X. Feng and J. M. Winters, "An interactive framework for personalized computer-assisted neurorehabilitation," Information Technology in Biomedicine, IEEE Transactions on, IEEE, vol. 11, no. 5, pp. 518-526, 2007.

[60] C. Roberto, P. Fabrizio, D. Carmen, M. Silvestro, C. Chiara, D. Paolo and M. Giuseppe, "Design strategies to improve patient motivation during robot-aided rehabilitation," J Neuroengineering Rehabil, vol. 4, no. 3, 2007.

[61] F. G. M. A. Q. Q. L. J. M. A. Adamovich SV, "Design of a complex virtual reality simulation to train finger motion for persons with hemiparesis: a proof of concept study.," J Neuroeng Rehabil., vol. 6, no. 28, pp. 1186 - 1743, 2009 Jul 17.

[62] J. W. Sensinger and R. F. Weir, "Non-Backdrivable Series Elastic Actuator For Use In A Prosthetic Elbow," in Myoelectric Symposium, 2005.

[63] R. Riener, Robot-aided rehabilitation of neural function in the upper extremities, Springer, 465-471, 2007 .

[64] N. Hogan, H. I. Krebs, J. Charnnarong, P. Srikrishna and A. Sharon, "MITMANUS: a workstation for manual therapy and training," in IEEE International Workshop on Robot and Human Communication, Proceedings, 161-165, 1992.

R. Loureiro, F. Amirabdollahian, S. Coote, E. Stokes and W. Harwin, "Using haptics technology to deliver motivational therapies in stroke patients: Concepts and initial pilot studies," in Proceedings of EuroHaptics, Birmingham, UK, 2001.

[66] R. Loureiro, F. Amirabdollahian, B. Driessen and W. Harwin, "A novel method for computing natural path for robot assisted movements in synthetic worlds," in Proc. Assisitive Technology-Added Value to the Quality of Life, 2001. 
[67] R. Loureiro, F. Amirabdollahian, M. Topping, B. Driessen and W. Harwin, "Upper limb robot mediated stroke therapy—GENTLE/s approach," Autonomous Robots, Springer, vol. 15, no. 1, pp. 35-51, 2003.

[68] R. Q. Van der Linde, P. Lammertse, E. Frederiksen and B. Ruiter, "The HapticMaster, a new high-performance haptic interface," in In Proc. Eurohaptics, 1$5,2002$.

[69] R. C. Loureiro, W. S. Harwin, K. Nagai and M. Johnson, "Advances in upper limb stroke rehabilitation: a technology push," Med.Biol.Eng.Comput., Springer, vol. 49, no. 10, pp. 1103-1118, 2011.

[70] P. S. Lum, H. Van der Loos, P. Shor and C. G. Burgar, "A robotic system for upper-limb exercises to promote recovery of motor function following stroke," in Proceedings Sixth Int. Conf. on Rehab. Robotics (ICORR 1999), Stanford, CA, USA, 1999.

[71] S. Hesse, G. Schulte-Tigges, M. Konrad, A. Bardeleben and C. Werner, "Robotassisted arm trainer for the passive and active practice of bilateral forearm and wrist movements in hemiparetic subjects," Arch.Phys.Med.Rehabil., Elsevier, vol. 84, no. 6, pp. 915-920, 2003.

[72] H. Krebs, N. Hogan, B. Volpe, M. Aisen, L. Edelstein and C. Diels, "Robot-aided Neuro-rehabilitation in Stroke: Three Year follow-up," in Proceedings of ICORR1999, pp 34-41, 1999.

[73] B. Volpe, H. Krebs, N. Hogan, L. Edelsteinn, C. Diels and M. Aisen, "Robot training enhanced motor outcome in patients with stroke maintained over 3 years," Neurology, AAN Enterprises, vol. 53, no. 8, pp. 1874-1874, 1999.

[74] "Journal of NeuroEngineering and Rehabilitation," [Online]. Available: http://www.jneuroengrehab.com. 
M. L. Aisen, H. I. Krebs, N. Hogan, F. McDowell and B. T. Volpe, "The effect of robot-assisted therapy and rehabilitative training on motor recovery following stroke," Arch.Neurol., Am Med Assoc, vol. 54, no. 4, p. 443, 1997.

[76] S. E. Fasoli, H. I. Krebs, J. Stein, W. R. Frontera, R. Hughes and N. Hogan, "Robotic therapy for chronic motor impairments after stroke: Follow-up results," Arch.Phys.Med.Rehabil., Elsevier, vol. 85, no. 7, pp. 1106-1111, 2004.

M. Ferraro, J. Palazzolo, J. Krol, H. Krebs, N. Hogan and B. Volpe, "Robot-aided sensorimotor arm training improves outcome in patients with chronic stroke," Neurology, AAN Enterprises, vol. 61, no. 11, pp. 1604-1607, 2003.

[78] N. Hogan, "A Robot for Wrist Rehabilitation-Characterization and Initial Results," in 8th International Conference on Rehabilitation Robotics PROCEEDINGS 한국지능시스템학회, Daejon, South Korea, 2003.

[79] S. Lum, S. L. Lehman and D. J. Reinkensmeyer, "The bimanual lifting rehabilitator: an adaptive machine for therapy of stroke patients," IEEE Transactions on Rehabilitation Engineering, IEEE, vol. 3, no. 2, pp. 166-174, 1995.

[80] P. S. Lum, D. J. Reinkensmeyer and S. L. Lehman, "Robotic assist devices for bimanual physical therapy: preliminary experiments," IEEE Transactions on Rehabilitation Engineering, IEEE, vol. 1, no. 3, pp. 185-191, 1993.

[81] P. S. Lum, H. Van der Loos, P. Shor and C. G. Burgar, "A robotic system for upper-limb exercises to promote recovery of motor function following stroke," in International conference on rehabilitation robotics (ICORR 1999), , Stanford, CA, USA, pp 235-239, 1999.

[82] P. S. Lum, C. G. Burgar, D. E. Kenney and H. M. Van der Loos, "Quantification of force abnormalities during passive and active-assisted upper-limb reaching movements in post-stroke hemiparesis," IEEE Transactions on Biomedical Engineering, IEEE, vol. 46, no. 6, pp. 652-662, 1999. 
P. S. Lum, C. G. Burgar, M. Van der Loos, P. C. Shor, M. Majmundar and R. Yap, "MIME robotic device for upper-limb neurorehabilitation in subacute stroke subjects: A follow-up study," Journal of rehabilitation research and development, REHIBILITATION RESEARCH \& DEVELOPMENT SERVICE, vol. 43, no. 5, p. 631, 2006.

[84] T. Nef and R. Riener, "ARMin-design of a novel arm rehabilitation robot," in 9th International Conference on Rehabilitation Robotics, ICORR 2005., pp: 57-60, 2005.

[85] M. Mihelj, T. Nef and R. Riener, "ARMin-toward a six DoF upper limb rehabilitation robot," in The First IEEE/RAS-EMBS International Conference on Biomedical Robotics and Biomechatronics. BioRob 2006. , pp: 1154-1159, 2006.

[86] T. Nef, M. Mihelj, G. Kiefer, C. Perndl, R. Muller and R. Riener, "ARMinExoskeleton for arm therapy in stroke patients," in IEEE 10th International Conference on Rehabilitation Robotics. ICORR 2007. IEEE., pp: 68-74, 2007.

[87] R. Sanchez, D. Reinkensmeyer, P. Shah, J. Liu, S. Rao, R. Smith, S. Cramer, T. Rahman and J. Bobrow, "Monitoring functional arm movement for home-based therapy after stroke," in 26th Annual International Conference of the IEEE. Engineering in Medicine and Biology Society. IEMBS'04., 2004. training with T-WREX after chronic stroke: preliminary results of a randomized controlled trial," in IEEE 10th International Conference on Rehabilitation Robotics. ICORR 2007., pp: 562-568, 2007.

[89] D. J. Reinkensmeyer and S. J. Housman, ""If I can't do it once, why do it a hundred times?": Connecting volition to movement success in a virtual environment motivates people to exercise the arm after stroke," in IEEE virtual rehabilitation, , , , Venice, Italy, pp: 44-48, 27-29 September. 

of the upper limb after acute stroke," Arch.Phys.Med.Rehabil., Elsevier, vol. 88, no. 2, pp. 142-149, 2007.

[91] K. Nagai, Y. Kojima, S. Yonemoto, T. Okubo, R. C. Loureiro and W. S. Harwin, "Structural design of an escort type rehabilitation robot for post-stroke therapies of upper-limb," in IEEE 10th International Conference on Rehabilitation Robotics. ICORR 2007, IEEE, pp: 1121-1128. 2007.

[92] Y. Ikegami, K. Nagai, R. C. Loureiro and W. S. Harwin, "Design of redundant drive joint with adjustable stiffness and damping mechanism to improve joint admittance," in IEEE International Conference on Rehabilitation Robotics. ICORR 2009. , pp: 202-210, 2009.

[93] K. Nagai, Y. Dake, Y. Shiigi, R. C. Loureiro and W. S. Harwin, "Design of redundant drive joints with double actuation using springs in the second actuator to avoid excessive active torques," in IEEE International Conference on Robotics and Automation (ICRA), pp: 805-812, 2010 .

[94] K. Nagai, Y. Shiigi, Y. Ikegami, R. Loureiro and W. S. Harwin, "Impedance control of redundant drive joints with double actuation," in IEEE International Conference on Robotics and Automation. ICRA'09, pp: 1528-1534, 2009.

[95] G. C. Burdea, C. Burdea and C. Burdea, Force and touch feedback for virtual reality, New York: Wiley, 1996.

[96] A. Kargov, C. Pylatiuk, H. Klosek, R. Oberle, S. Schulz and G. Bretthauer, "Modularly designed lightweight anthropomorphic robot hand," in Multisensor Fusion and Integration for Intelligent Systems, IEEE International Conference, pp: 155-159, 2006.

[97] N. Tsujiuchi, T. Koizumi, S. Nishino, H. Komatsubara, T. Kudawara and M. Hirano, "Development of pneumatic robot hand and construction of master-slave 
system," Journal of system design and dynamics, J-STAGE, vol. 2, no. 6, pp. 13061315, 2008.

[98] L. Tan, S. Xie, I. Lin and T. Lin, "Development of a multifingered robotic hand," in International Conference on Information and Automation, 2009. ICIA'09., 2009.

[99] I. Yamano and T. Maeno, "Five-fingered robot hand using ultrasonic motors and elastic elements," in Proceedings of the 2005 IEEE International Conference on Robotics and Automation. ICRA 2005., 2005.

[100] T. Maeno and T. Hino, "Miniature five-fingered robot hand driven by shape memory alloy actuators," in Proceedings of the 12th IASTEDInternational Conference, Robotics and Applications, Hawaii, USA, 2006.

[101] V. Bundhoo, E. Haslam, B. Birch and E. J. Park, "A shape memory alloy-based tendon-driven actuation system for biomimetic artificial fingers, part I: design and evaluation," Robotica, Cambridge Univ Press, vol. 27, no. 1, pp. 131-146, 2009.

[102] N. H. Chuc, J. K. Park, N. H. L. Vuong, D. Kim, J. C. Koo, Y. Lee, J.-D. Nam and H. R. Choi, "Multi-jointed robot finger driven by artificial muscle actuator," in IEEE International Conference on Robotics and Automation. ICRA'09. , 2009.

[103] G. Andrikopoulos, G. Nikolakopoulos and S. Manesis, "A survey on applications of pneumatic artificial muscles," in Control \& Automation (MED), 2011 19th Mediterranean Conference on. IEEE, 2011.

[104] R. Sanchez Jr, E. Wolbrecht, R. Smith, J. Liu, S. Rao, S. Cramer, T. Rahman, J. Bobrow and D. Reinkensmeyer, "A pneumatic robot for re-training arm movement after stroke: Rationale and mechanical design," in Rehabilitation Robotics, 2005. ICORR 2005. 9th International Conference on. IEEE, 2005. 
[105] E. Koeneman, R. Schultz, S. Wolf, D. Herring and J. Koeneman, "A pneumatic muscle hand therapy device," in Engineering in Medicine and Biology Society, 2004. IEMBS'04. 26th Annual International Conference of the IEEE, 2004.

[106] Y. Umetani, Y. Yamada, T. Morizono, T. Yoshida and S. Aoki, "Skil Mate" wearable exoskeleton robot," in Systems, Man, and Cybernetics, 1999. IEEE SMC'99 Conference Proceedings. 1999 IEEE International Conference on. .

[107] J. He, E. Koeneman, R. Schultz, D. Herring, J. Wanberg, H. Huang, T. Sugar, R. Herman and J. Koeneman, "RUPERT: a device for robotic upper extremity repetitive therapy," in Engineering in Medicine and Biology Society, 2005. IEEE-EMBS 2005. 27th Annual International Conference of the. IEEE.

[108] J. He, E. Koeneman, R. Schultz, H. Huang, J. Wanberg, D. Herring, T. Sugar, R. Herman and J. Koeneman, "Design of a robotic upper extremity repetitive therapy device," in Rehabilitation Robotics, 2005. ICORR 2005. 9th International Conference on. IEEE.

[109] D. Sasaki, T. Noritsugu and M. Takaiwa, "Development of active support splint driven by pneumatic soft actuator (ASSIST)," in Proceedings of the 2005 IEEE International Conference on Robotics and Automation, 2005. ICRA 2005. IEEE, pp. 520-525, 2005.

[110] H. Kobayashi, H. Nozaki and T. Tsuji, "Development of power assist system for caregiver by muscle suit," in echatronics and Automation, 2007. ICMA 2007. International Conference on. IEEE, 2007.

[111] D. Caldwell, G. Medrano-Cerda and M. Goodwin, "Braided pneumatic actuator control of a multi-jointed manipulator," in Systems, Man and Cybernetics, 1993.'Systems Engineering in the Service of Humans', Conference Proceedings., International Conference on. IEEE, 1993. 
[112] V. L. Nickel, J. Perry and A. L. Garrett, "Development of useful function in the severely paralyzed hand," The Journal of Bone \& Joint Surgery, The Journal of Bone and Joint Surgery, vol. 45, no. 5, pp. 933-952, 1963.

[113] C.-P. Chou and B. Hannaford, "Measurement and modeling of McKibben pneumatic artificial muscles," Robotics and Automation, IEEE Transactions on, IEEE, vol. 12, no. 1, pp. 90-102, 1996.

[114] H. Kobayashi and K. Hiramatsu, "Development of muscle suit for upper limb," in Robotics and Automation, 2004. Proceedings. ICRA'04. 2004 IEEE International Conference on. IEEE., 2004.

[115] A. r. w. a. r. e. i. V. Reality, "Arm rehabilitation with a robotic exoskeleleton in Virtual Reality," in Rehabilitation Robotics. IEEE 10th International Conference ICORR 2007, IEEE, pp: 631-642, 2007.

[116] L.-W. Tsai, Robot analysis: the mechanics of serial and parallel manipulators, New York, U.S.A.: John Wiley \& Sons Inc., 1999.

[117] G. Johnson, D. Carus, G. Parrini, S. Marchese and R. Valeggi, "The design of a five-degree-of-freedom powered orthosis for the upper limb," Proc.Inst.Mech.Eng.Part H J.Eng.Med., SAGE Publications, vol. 215, no. 3, pp. 275284, 2001.

[118] J. Rosen and J. C. Perry, "Upper limb powered exoskeleton," International Journal of Humanoid Robotics, World Scientific, vol. 4, no. 3, pp. 529-548, 2007.

[119] J. C. Perry, J. Rosen and S. Burns, "Upper-limb powered exoskeleton design," Mechatronics, IEEE/ASME Transactions, IEEE, vol. 12, no. 4, pp. 408-417, 2007.

[120] S. J. Ball, I. E. Brown and S. H. Scott, "MEDARM: a rehabilitation robot with 5DOF at the shoulder complex," in Advanced intelligent mechatronics, IEEE/ASME international conference, pp. 1-6, 2007. 
[121] T. Nef, M. Mihelj, G. Colombo and R. Riener, "ARMin-robot for rehabilitation of the upper extremities," in Robotics and Automation. ICRA 2006. Proceedings 2006 IEEE International Conference, pp: 3152-3157,2006.

[122] T. Nef and R. Riener, "Shoulder actuation mechanisms for arm rehabilitation exoskeletons," in Biomedical Robotics and Biomechatronics. BioRob 2008. 2nd IEEE RAS \& EMBS International Conference, pp: 862-868, 2008.

[123] L. Lucas, M. DiCicco and Y. Matsuoka, "An EMG-controlled hand exoskeleton for natural pinching," Journal of Robotics and Mechatronics, FUJI TECHNOLOGY PRESS LTD., vol. 16, pp. 482-488, 2004.

[124] A. Wege and G. Hommel, "Development and control of a hand exoskeleton for rehabilitation of hand injuries," in Intelligent Robots and Systems, (IROS 2005). 2005 IEEE/RSJ International Conference, pp. 3046-3051, 2005.

[125] T. Wurtz, C. May, B. Holz, C. Natale, G. Palli and C. Melchiorri, "The twisted string actuation system: Modeling and control," in IEEE/ASME International Conference on Advanced Intelligent Mechatronics (AIM), pp: 1215-1220, 2010.

[126] G. Palli, C. Natale, C. May, C. Melchiorri and T. Wurtz, "Modeling and control of the twisted string actuation system," Mechatronics, IEEE/ASME Transactions on, vol. 18, no. 2, pp. 664 - 673, 2012.

[127] "DEXMART Project website. (2008). [Online]. Available:," [Online]. Available: http://www.dexmart.eu/. [Accessed 62 2014].

[128] G. Berselli, G. Borghesan, M. Brandi, C. Melchiorri, C. Natale, G. Palli, S. Pirozzi and G. Vassura, "Integrated mechatronic design for a new generation of robotic hands," in IFAC Symposium on Robot Control, Gifu, 2009. 
[129] T. Sonoda and I. Godler, "Multi-fingered robotic hand employing strings transmission named "Twist Drive"," in Intelligent Robots and Systems (IROS), 2010 IEEE/RSJ International Conference on. IEEE, pp:2733-2738, 2010.

[130] I. Godler and T. Sonoda, "A five fingered robotic hand prototype by using twist drive," in Robotics (ISR) 41st International Symposium on and 2010 6th German Conference on Robotics (ROBOTIK), 2010.

[131] T. Sonoda and I. Godler, "Position and force control of a robotic finger with twisted strings actuation," in Advanced Intelligent Mechatronics (AIM), 2011 IEEE/ASME International Conference on , pp. 611-616, 2011.

[132] I. Godler and T. Sonoda, "Performance evaluation of twisted strings driven robotic finger," in Ubiquitous Robots and Ambient Intelligence (URAI), 2011 8th International Conference on, pp. 542-547, 2011.

[133] J. J. Guzek, C. Petersen, S. Constantin and H. Lipson, "Mini Twist: A Study of Long-Range Linear Drive by String Twisting," J. Mechanisms Robotics, vol. 4, no. 1, 2012.

[134] D. Aukes, S. Kim, P. Garcia, A. Edsinger and M. R. Cutkosky, "Selectively compliant underactuated hand for mobile manipulation," in Robotics and Automation (ICRA), 2012 IEEE International Conference on, 2012.

[135] Y. J. Shin, H. J. Lee, K. Kim and S. Kim, "A Robot Finger Design Using a DualMode Twisting Mechanism to Achieve High-Speed Motion and Large Grasping Force," Robotics, IEEE Transactions on, vol. 28, no. 6, pp. 1398 - 1405, 2012.

[136] R. Gopura and K. Kiguchi, "Mechanical designs of active upper-limb exoskeleton robots: State-of-the-art and design difficulties," in IEEE International Conference on Rehabilitation Robotics. ICORR 2009., 2009.

[137] A. H. Stienen, E. E. Hekman, F. C. Van der Helm, G. B. Prange, M. J. Jannink, A. M. Aalsma and H. Van der Kooij, "Dampace: dynamic force-coordination trainer 
for the upper extremities," in IEEE 10th International Conference on Rehabilitation Robotics. ICORR 2007. , 2007.

[138] E. Koeneman, R. Schultz, S. Wolf, D. Herring and J. Koeneman, "A pneumatic muscle hand therapy device," in 26th Annual International Conference of the IEEE Engineering in Medicine and Biology Society. IEMBS'04., 2004.

[139] C. Takahashi, L. Der-Yeghiaian, V. Le and S. Cramer, "A robotic device for hand motor therapy after stroke," in 9th International Conference on Rehabilitation Robotics. ICORR 2005, 2005.

[140] Y. S. Kim, J. Lee, S. Lee and M. Kim, "A force reflected exoskeleton-type masterarm for human-robot interaction," Systems, Man and Cybernetics, Part A: Systems and Humans, IEEE Transactions on, IEEE, vol. 35, no. 2, pp. 198-212, 2005.

[141] A. Montagner, A. Frisoli, L. Borelli, C. Procopio, M. Bergamasco, M. C. Carboncini and B. Rossi, "A pilot clinical study on robotic assisted rehabilitation in VR with an arm exoskeleton device," in Virtual Rehabilitation. IEEE., 2007.

[142] C. Carignan, J. Tang, S. Roderick and M. Naylor, "A configuration-space approach to controlling a rehabilitation arm exoskeleton," in IEEE 10th International Conference on Rehabilitation Robotics. ICORR 2007, 2007.

[143] J. Stein, K. Narendran, J. McBean, K. Krebs and R. Hughes, "Electromyographycontrolled exoskeletal upper-limb-powered orthosis for exercise training after stroke," Am.J.Phys.Med.Rehabil., vol. 86, no. 4, pp. 255-261, 2007.

[144] R. Sanchez Jr, E. Wolbrecht, R. Smith, J. Liu, S. Rao, S. Cramer, T. Rahman, J. Bobrow and D. Reinkensmeyer, "A pneumatic robot for re-training arm movement after stroke: Rationale and mechanical design," in 9th International Conference on Rehabilitation Robotics. ICORR 2005, 2005.

[145] T. G. Sugar, J. He, E. J. Koeneman, J. B. Koeneman, R. Herman, H. Huang, R. S. Schultz, D. Herring, J. Wanberg and S. Balasubramanian, "Design and control of 
RUPERT: a device for robotic upper extremity repetitive therapy," IEEE Transactions on Neural Systems and Rehabilitation Engineering, IEEE., vol. 15, no. 3, pp. 336-346, 2007.

A. Merians, H. Poizner, R. Boian, G. Burdea and S. Adamovich, "Sensorimotor training in a virtual reality environment: does it improve functional recovery poststroke?," Neurorehabil.Neural Repair, vol. 20, no. 2, pp. 252-267, 2006.

[147] S. Moubarak, M. T. Pham, T. Pajdla and T. Redarce, "Design and modeling of an upper extremity exoskeleton," in World Congress on Medical Physics and Biomedical Engineering. Springer Berlin Heidelberg, Munich, Germany, September 7-12, 2009.

[148] B.-C. Tsai, W.-W. Wang, L.-C. Hsu, L.-C. Fu and J.-S. Lai, "An articulated rehabilitation robot for upper limb physiotherapy and training," in IEEE/RSJ International Conference on Intelligent Robots and Systems (IROS), 2010.

[149] M. Ngai, "Design of a Forearm Exoskeleton for Supination/Pronation Assistance in Daily Activities," McMaster University, Hamilton. Canada, 2010.

[150] R. Zheng and J. Li, "Kinematics and workspace analysis of an exoskeleton for thumb and index finger rehabilitation," in IEEE International Conference on Robotics and Biomimetics (ROBIO), 2010, 2010.

[151] W. Yu and J. Rosen, "A novel linear PID controller for an upper limb exoskeleton," in 49th IEEE Conference on Decision and Control (CDC), 2010.

[152] L. I. Ammar, B. Y. Kaddouh, M. K. Mohanna and I. H. Elhajj, "SAS: SMA Aiding Sleeve," in IEEE International Conference on Robotics and Biomimetics (ROBIO), 2010.

[153] P. Garrec, "Design of an anthropomorphic upper limb exoskeleton actuated by ball-screws and cables," University" Politehnica" of Bucharest Scientific Bulletin, 
Series D: Mechanical Engineering, Politehnica University of Bucharest, vol. 72, no. 2, pp. 23-34, 2010.

[154] J. ZHANG, H. FU, Y. DONG, Y. ZHANG, C. YANG and Y. CHEN, "Novel 6DOF Wearable Exoskeleton Arm with Pneumatic Force-Feedback for Bilateral Teleoperation," Chin.J.Mech.Eng., vol. 22, no. 3, 2012.

[155] R. Gopura and K. Kiguchi, "Development of a 6dof exoskeleton robot for human upper-limb motion assist," in 4th International Conference on Information and Automation for Sustainability. ICIAFS 2008. , 2008.

$[156$
Wikipedia,
"Wikipedia,"
[Online].
Available:

http://en.wikipedia.org/wiki/Upper_limb. [Accessed 239 2013].

[157]

E. J. McCormick, Human Factors Engineering, 3rd ed., New York: McGrawHill, 1970.

[158] C. Carignan, M. Liszka and S. Roderick, "Design of an arm exoskeleton with scapula motion for shoulder rehabilitation," in 12th International Conference on Advanced Robotics. ICAR'05. Proceedings, IEEE, 524-531, 2005.

[159]
"cnx,"
[Online].
Available:

http://cnx.org/content/m45990/latest/body+planes+HD\&safe=off\&tbm=isch\&facrc= _\&imgdii=_\&imgrc=4BrgzNiuaq2z1M\%. [Accessed 102 2014].

[160] F. Martini, M. Timmons and R. Tallitsch, Human Anatomy 4 th Ed, Pearson Education Inc., 2003.

[161] A. Engín, "On the biomechanics of the shoulder complex," J.Biomech., Elsevier, vol. 13 , no. 7 , pp. 575-590, 1980.

$[162$

"Shoulderdoc," [Online].

Available:

http://www.shoulderdoc.co.uk/article.asp?article=1177. [Accessed 102 2014]. 
http://conornordengren.com/2011/10/14/the-shoulder-girdle-part-1-bones-andjoints-2/. [Accessed 102 2014].

[164]
"smartdraw,"
[Online].
Available: https://www.smartdraw.com/examples/view/physical+therapy+\%26+rehabilitation+ -+range+of+motion+-+shoulder/. [Accessed 102 2014].

Available: http://www.smartdraw.com/examples/view/physical+therapy+\%26+rehabilitation++range+of+motion+-+elbow+\%26+shoulder/. [Accessed 102 2014].

[166] R. C. Schafer and J. Anderson, Clinical biomechanics: musculoskeletal actions and reactions, Baltimore: Williams \& Wilkins , 1987.
S.
R.
C., $\quad$ "chiro.org,"
[Online].
Available: http://www.chiro.org/ACAPress/The_Upper_Extremity_CB.html\#Elbow_and_Fore arm. [Accessed 102 2014].

H. M. Clarkson, Joint motion and function assessment: a research-based practical guide, Lippincott Williams \& Wilkins, 2005.

[169] "www.merriam-webster.com," [Online]. Available: http://visual.merriamwebster.com/human-being/anatomy/skeleton/types-synovial-joints_1.php. [Accessed $2832014]$.

[170] J. Sagano, D. Magee and M. Katayose, "The Effect of Glenohumeral Rotation on Scapular Upward Rotation in Different Positions of Scapular-Plane Elevation.," J.Sport Rehab., vol. 15, no. 2, pp. 144-155, 2006.

[171] P. Borsa, M. Timmons and E. Sauers, "Scapular-Positioning Patterns During Humeral Elevation in Unimpaired Shoulders," J.Athl Train., vol. 38, no. 1, pp. 12-17, 2003. 
[172] N. P. Palastanga and R. Soames, Anatomy and human movement: structure and function, Churchill Livingstone, 2011, Churchill Livingstone.

[173] Wikipedia, "Wikipedia.org," [Online]. Available: http://en.wikipedia.org/wiki/Elbow. [Accessed 239 2013].

[174] J. London, "Kinematics of the elbow," J.Bone Joint Surg.Am., UNITED STATES, vol. 63 , no. 4 , pp. 529-535, 1981.

[175] I. Kapandji, The Physiology of the Joints. Volume 1: Upper Limb, Churchill Livingstone, 1974.

[176] Anatomisty, "anatomisty.com," [Online]. Available: http://anatomisty.com/anatomy-sistems/hinge-joint-2/. [Accessed 239 2013].

[177] D. J. Magee, Orthopaedic physical assessment, Elsevier Health Sciences, 2008.

[178] D. A. Winter, Biomechanics and motor control of human movement, New Jersey: John Wiley \& Sons, 2009.

[179] A. A. Association, "What is Anthropology?," [Online]. Available: http://www.aaanet.org/about/whatisanthropology.cfm. [Accessed 91 2014].

[180] "Centers for Disease Control and Prevention," [Online]. Available: http://www.cdc.gov/niosh/topics/anthropometry/. [Accessed 91 2014].

[181] W. T. Dempster, "Space requirements of the seated operator: geometrical, kinematic, and mechanical aspects of the body, with special reference to the limbs," 1955.

[182] W. T. Dempster, W. C. Gabel and W. J. Felts, "The anthropometry of the manual work space for the seated subject," Am.J.Phys.Anthropol., vol. 17, no. 4, pp. 289-317, 1959. 
[183] R. Contini, "Body Segment Parameters, Part II1," Artif.Limbs, vol. 1, 1972.

[184] R. Drillis, R. Contini and M. Bluestein, "Body segment parameters," Artif.Limbs, vol. 8, no. 1, pp. 44-66, 1964.

[185] R. A. R. C. Gopura, K. Kiguchi and Y. Li, "SUEFUL-7: a 7DOF upper-limb exoskeleton robot with muscle-model-oriented EMG-based control," in IEEE/RSJ International Conference on Intelligent Robots and Systems. IROS 2009., IEEE, 1126-1131, 2009.

[186] K. Kiguchi, K. Iwami, M. Yasuda, K. Watanabe and T. Fukuda, "An exoskeletal robot for human shoulder joint motion assist," IEEE/ASME Transactions on Mechatronics, IEEE, vol. 8, no. 1, pp. 125-135, 2003.

[187] J. C. Perry, J. Rosen and S. Burns, "Upper-limb powered exoskeleton design," IEEE/ASME Transactions on Mechatronics, IEEE, vol. 12, no. 4, pp. 408-417, 2007.

[188] T. Nef, M. Mihelj, G. Colombo and R. Riener, "ARMin-robot for rehabilitation of the upper extremities," in Proceedings 2006 IEEE International Conference on Robotics and Automation. ICRA 2006, IEEE, pp 3152-3157, 2006.

[189] J. London, "Kinematics of the elbow," J.Bone Joint Surg.Am., UNITED STATES, vol. 63, no. 4, pp. 529-535, 1981.

[190] C. Yang, J. Zhang, Y. Chen, Y. Dong and Y. Zhang, "A review of exoskeletontype systems and their key technologies," Proc.Inst.Mech.Eng.Part C, SAGE Publications, vol. 222, no. 8, pp. 1599-1612, 2008.

[191] R. L. Sacco, E. J. Benjamin, J. P. Broderick, M. Dyken, J. D. Easton, W. M. Feinberg, L. B. Goldstein, P. B. Gorelick, G. Howard and S. J. Kittner, "Risk factors," Stroke, Am Heart Assoc, vol. 28, no. 7, pp. 1507-1517, 1997. 
[192] M. A. McDowell, C. D. Fryar and C. L. Ogden, "Anthropometric reference data for children and adults: United States, 1988-1994.," Vital and health statistics.Series 11, Data from the national health survey, vol. 249, pp. 1-68, 2009.

[193] D. C. Boone and S. P. Azen, "Normal range of motion of joints in male subjects," The Journal of Bone \& Joint Surgery, The Journal of Bone and Joint Surgery, vol. 61, no. 5, pp. 756-759, 1979.

[194] "indespension," [Online]. Available: http://www.indespension.co.uk/TrailerParts/Alko-Cables/BR062.html. [Accessed 283 2014].

[195] P. Korovessis, G. Koureas and Z. Papazisis, "Correlation between backpack weight and way of carrying, sagittal and frontal spinal curvatures, athletic activity, and dorsal and low back pain in schoolchildren and adolescents," J.Spinal.Disord.Tech., United States, vol. 17, no. 1, pp. pp. 33-40, 2004.

[196] D. Chow, K. Leung and A. Holmes, "Changes in spinal curvature and proprioception of schoolboys carrying different weights of backpack," Ergonomics, Taylor \& Francis, vol. 50, no. 12, pp. 2148-2156, 2007.

[197] J. Strickland, "howstuffworks," [Online]. Available: http://science.howstuffworks.com/gimbal1.htm. [Accessed 102 2014].

[198] J. Aizawa, T. Masuda, T. Koyama, K. Nakamaru, K. Isozaki, A. Okawa and S. Morita, "Three-dimensional motion of the upper extremity joints during various activities of daily living," J.Biomech., Elsevier, vol. 43, no. 15, pp. 2915-2922, 2010.

[199] P. Raiss, O. Rettig, S. Wolf, M. Loew and P. Kasten, "Range of motion of shoulder and elbow in activities of daily life in 3D motion analysis," Z.Orthop.Unfall, Germany, vol. 145, no. 4, pp. 493-498, 2007. 
[200] D. Magermans, E. Chadwick, H. Veeger and F. Van Der Helm, "Requirements for upper extremity motions during activities of daily living," Clin.Biomech., vol. 20, no. 6, pp. 591-599, 2005, Elsevier.

[201] G. Wu, F. C. Van der Helm, H. Veeger, M. Makhsous, P. Van Roy, C. Anglin, J. Nagels, A. R. Karduna, K. McQuade and X. Wang, "ISB recommendation on definitions of joint coordinate systems of various joints for the reporting of human joint motion - Part II: shoulder, elbow, wrist and hand," J.Biomech., Elsevier, vol. 38, no. 5, pp. 981-992, 2005.

[202] M. G. Kloosterman, G. J. Snoek, M. Kouwenhoven, A. V. Nene and M. J. Jannink, "Influence of gravity compensation on kinematics and muscle activation patterns during reach and retrieval in subjects with cervical spinal cord injury: an explorative study.," Journal of Rehabilitation Research \& Development, vol. 47, no. $7,2010$.

[203] T. Würtz, C. May, B. Holz, C. Natale, G. Palli and C. Melchiorri, "The twisted string actuation system: Modeling and control," in Advanced Intelligent Mechatronics (AIM), 2010 IEEE/ASME International Conference on. IEEE, pp. 1215-1220, 2010.

[204] J. J. Guzek, S. Constantin, H. Lipson and C. Petersen, "Mini Twist: A Study of Long-Range Linear Drive by String Twisting," Journal of mechanisms and robotics, American Society of Mechanical Engineers, vol. 4, no. 1, 2012.

[205] S. S. Rao and F. F. Yap, Mechanical vibrations, 4th Edition, New York: AddisonWesley, 1995.

[206] "Amazon," [Online]. Available: http://www.amazon.com/ASR-TacticalTechnora-Composite-Survival/dp/B004GBGVYS/ref=sr_1_2?s=sportinggoods\&ie=UTF8\&qid=1376189747\&sr=1-2\&keywords=ultra+thin+rope. [Accessed 283 2014]. 
[207] R. C. Goertz, "Fundamentals of general purpose remote manipulators," Nucleonics, vol. 10, no. 11, pp. 36-42, 1952.

[208] N. Tsujiuchi, T. Koizumi, S. Nishino, H. Komatsubara, T. Kudawara and M. Hirano, "Development of pneumatic robot hand and construction of master-slave system," Journal of system design and dynamics, vol. 2, pp. 1306-1315, 2008.

[209] K. Hirai, M. Hirose, Y. Haikawa and T. Takenaka, "The development of Honda humanoid robot," in IEEE International Conference on Robotics and Automation, 1998.

[210] T. Mouri, H. Kawasaki and K. Umebayashi, "Developments of new anthropomorphic robot hand and its master slave system," in IEEE/RSJ International Conference on Intelligent Robots and Systems.(IROS 2005), 2005.

[211] K. Tadano and K. Kawashima, "Development of a master slave system with force sensing using pneumatic servo system for laparoscopic surgery," in IEEE International Conference on Robotics and Automation, 2007.

[212] N. Abolhassani and R. V. Patel, "Teleoperated master-slave needle insertion," The International Journal of Medical Robotics and Computer Assisted Surgery, Wiley Online Library, vol. 5, no. 4, pp. 398-405, 2009.

[213] M. Tavakoli, R. PateI and M. Moallem, "Design issues in a haptics-based masterslave system for minimally invasive surgery," in IEEE International Conference on Robotics and Automation. ICRA'04. , 2004.

[214] M. R. V. P. a. M. M. Tavakoli, "Design issues in a haptics-based master-slave system for minimally invasive surgery," in IEEE International Conference on Robotics and Automation. ICRA'04, 2004.

[215] L. Huang, "Robotics Locomotion Control," University of California, Berkeley, ProQuest, UMI Dissertations Publishing, 3210624., 2005. 
[216] A. Rovers, M. Steinbuch and I. Lammerts, "Design of a robust master-slave controller for surgery applications with haptic feedback," Eindhoven University of Technology, DCT Report, 2003.

[217] A. Sherman, M. C. Çavusoglu and F. Tendick, "Comparison of teleoperator control architectures for palpation task," in Proceedings of the ASME Dynamic Systems and Control Division, part of the ASME Internatioal Mechanical Engineering Congress and Exposition (IMECE 2000), 2000.

[218] C. Ganesh, B. Abhi, V. Anand, S. Aravind, R. Nandhini and S. Patnaik, "DC Position Control System-Determination of Parameters and Significance on System Dynamics," ACEEE International Journal on Electrical and Power Engineering, vol. 3, no. 1, pp. 1-5, 2012.

[219] "DC Motor Speed: System Modeling," [Online]. Available: http://ctms.engin.umich.edu/CTMS/index.php?example=MotorSpeed\&section=Syst emModeling. [Accessed 1312 2013].

[220] K. J. Åström and T. Hägglund, Advanced PID control, ISA-The Instrumentation, Systems, and Automation Society; Research Triangle Park, NC 27709, 2006.

[221] M. Cheng, G. Guo, L. Banta and E. Bakhoum, "Identification of Arm Locomotion and Controller Synthesis for Assistive Robotic Systems," ICIC Express Letters ICIC International, vol. 6, no. 10, pp. 2659-2664, 2012.

[222] E. J. Haug, Computer aided kinematics and dynamics of mechanical systems, Boston: Allyn and Bacon, 1989.

[223] "www.pololu.com," [Online]. Available: http://www.pololu.com/product/1442. [Accessed 243 2014]. 
http://www.washington.edu/doit/Faculty/Strategies/Disability/Mobility. [Accessed $1572012]$.

[225] N. I. o. N. D. a. Stroke, "Brain Basics: Preventing Stroke," National Institutes of Health, Bethesda, 2013.

[226] V. L. Roger, A. S. Go, D. M. Lloyd-Jones, R. J. Adams, J. D. Berry, T. M. Brown and e. al., "Heart Disease and Stroke Statistics_-2011 Update1. About 1. About These Statistics2. American Heart Association's 2020 Impact Goals3. Cardiovascular Diseases4. Subclinical Atherosclerosis5. Coronary Heart Disease, Acute Coronary Syndrome, and...," Circulation, vol. 123, no. 4 , pp. e18-e209., 2011.

[227] E. Jauch, B. Cucchiara, O. Adeoye, W. Meurer, J. Brice, Y. Chan, N. Gentile and M. F. Hazinski, "Part 11: Adult Stroke," Circulation, Am Heart Assoc, vol. 122, no. 18, pp. S818-S828, 2010.

[228] N. S. Association, "Stroke 101," [Online]. Available: http://www.stroke.org/site/DocServer/STROKE_101_Fact_Sheet.pdf?docID=4541. [Accessed 512 2011.].

Anatomisty, "Anatomisty.com," [Online]. Available: http://anatomisty.com/anatomy-sistems/ball-and-socket-joint-2/. [Accessed $23 \quad 9$ 2013].

Wikipedia, "Wikipedia.org," [Online]. Available: http://en.wikipedia.org/wiki/File:Gray326.png. [Accessed 239 2013].

[231] Wikipedia, "Wikipedia.com," [Online]. Available: http://en.wikipedia.org/wiki/File:Gray412.png. [Accessed 239 2013]. 
[232]

Wikipedia, "wikipedia.org," [Online]. Available: http://en.wikipedia.org/wiki/File:RightHumanPosteriorDistalRadiusUlnaCarpals.jpg . [Accessed 239 2013].

[233] Wikipedia, "wikipedia.org," [Online]. Available: http://en.wikipedia.org/wiki/File:RightHumanAnteriorDistalRadiusUlnaCarpals.jpg. [Accessed 239 2013].

[234] C. Neu, J. Crisco and S. Wolfe, "In vivo kinematic behavior of the radio-capitate joint during wrist flexion-extension and radio-ulnar deviation," J.Biomech., Elsevier, vol. 34, no. 11, pp. 1429-1438, 2001.

[235] Y. Youm and A. E. Flatt, "Design of a total wrist prosthesis," Ann.Biomed.Eng., Springer, vol. 12, no. 3, pp. 247-262, 1984.

[236] K. Kiguchi, K. Iwami, M. Yasuda, K. Watanabe and T. Fukuda, "An exoskeletal robot for human shoulder joint motion assist," IEEE/ASME Transactions on Mechatronics, IEEE, vol. 8, no. 1, pp. 125-135, 2003.

[237] E. Papadopoulos and G. Patsianis, "Design of an Exoskeleton Mechanism for the Shoulder Joint," in Proc.Twelfth World Congr.in Mechanism and Machine Sci.,, Besancon, France, pp: 1-6, 2007.

[238] R. A. R. C. Gopura and K. Kiguchi, "An Exoskeleton Robot for Human Forearm and Wrist Motion Assist," Journal of Advanced Mechanical Design, Systems, and Manufacturing, J-STAGE, vol. 2, no. 6, pp. 1067-1083, 2008.

[239] D. Sasaki, T. Noritsugu, M. Takaiwa and H. Yamamoto, "Wearable power assist device for hand grasping using pneumatic artificial rubber muscle," in 13th IEEE International Workshop on Robot and Human Interactive Communication. ROMAN 2004. , Sapporo, Hokkaido, Japan, pp. 420-425, 2004. 
[240] O. Katsuhiko, Modern Control Engineering, 5th edition, Englewood Cliffs, NJ: Prentice-Hall, 2010. 


\section{Appendix A: Kinetic Modeling}

\section{A.1. Introduction}

In this chapter a 2 DOF model for upper extremities was presented, which has one degree of freedom at shoulder and one for the elbow. First, the location of the center of gravity of each limb were determined and then the derivative of the position with respect to time were taken to find the velocity at each point. Then the Lagrange functions were formed using the kinetic energy, potential energy and external forces.

\section{A.2. Euler-Lagrange Method and Modeling of the Upper Extremities}

In this section a mass-spring-damper model was presented for upper extremities. In this model the shoulder and elbow joints had only one degree of freedom each, i.e. the motion is constrained to the parasagittal plane through the shoulder/elbow. The Lagrangian function is the difference 
between the kinetic energy and potential energy of a system, so the first step to construct a Lagrangia model is to determine the kinetic energy, potential energy and external forces on the components. This system includes springs and dampers at the shoulder and elbow joints and rigid masses for the upper arm, fore arm and hand, as shown in Table A. 1.

Table A. 1. Parameters used in modeling

\begin{tabular}{|c|c|c|c|}
\hline$m_{u}$ & Upper arm mass & $I_{u}$ & Upper arm moment of inertia about its $C G$ \\
\hline$m_{f}$ & Forearm mass & $I_{f}$ & Forearm moment of inertia about its $C G$ \\
\hline$m_{h}$ & Hand mass & $C G_{u}$ & Distance of the upper arm $C G$ from proximal \\
\hline$\theta_{u}$ & Upper arm angle & $C G_{f}$ & Distance of the forearm $C G$ from proximal \\
\hline$\theta_{f}$ & Forearm angle & $C G_{h}$ & Distance of the palm $C G$ from proximal \\
\hline$v_{u}$ & Velocity of the $C G$ of the upper arm & $k_{u}$ & Spring constant at shoulder joint \\
\hline$v_{f}$ & Velocity of the $C G$ of the forearm & $k_{f}$ & Spring constant at elbow joint \\
\hline$v_{h}$ & Velocity of the $C G$ of the hand & $c_{u}$ & Damper constant at shoulder joint \\
\hline$L_{u}$ & Upper arm segment length & $c_{f}$ & Damper constant at elbow joint \\
\hline$L_{f}$ & $\begin{array}{l}\text { Forearm segment length from elbow to palm } \\
C G\end{array}$ & & \\
\hline
\end{tabular}




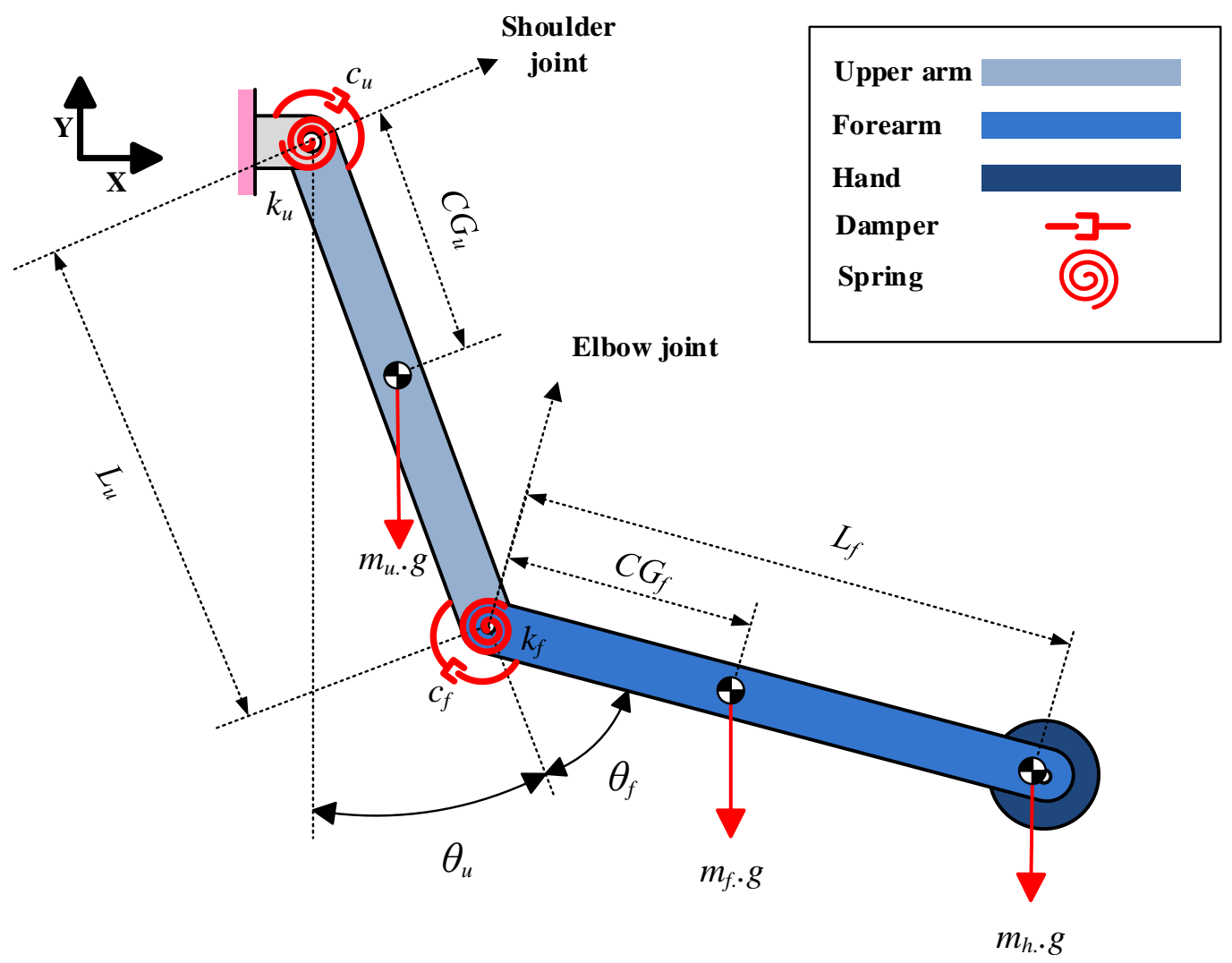

Figure A. 1. Schematic of the upper extremities

Forces on the system included gravity, which was considered as a potential energy, and external forces such as friction, damping forces and torques.

To derive the equations of motion using Lagrange method, the Lagrangian function must be formed and then derive the $n$ equations of a $n$-degree of freedom system using the Euler-Lagrange equation. Thus, the Lagrangian function as the difference between the kinetic energy and potential energy of the system and the Euler-Lagrange equation with no damping and no external forces is:

$$
\begin{gathered}
L=T-U, \\
\frac{d}{d t}\left(\frac{\partial L}{\partial \dot{q}_{i}}\right)-\frac{\partial L}{\partial q_{i}}=0 \quad, \quad i=1,2, \ldots, n,
\end{gathered}
$$

Where $q$ is the coordinate vector $[x, y, \theta]^{\mathrm{T}}$ and the subscript $i$ refers to the individual masses in the system, i.e. the upper arm, forearm and hand. 
If there exist generalized forces such as torques, friction or other sources of dissipation, $Q_{i}$ as the corresponding term must be included. The equation is rewritten:

$$
\frac{d}{d t}\left(\frac{\partial L}{\partial \dot{q}_{i}}\right)-\frac{\partial L}{\partial q_{i}}=Q_{i} \quad, \quad i=1,2, \ldots, n,
$$

In the special case of viscous damping, which is damping proportional to velocity, the Rayleigh Dissipation Function (RDF) can be used. Thus, the contribution of a damper of magnitude $c$ between masses $i$ and $j$ is:

$$
F_{i j}=\frac{1}{2} c\left(\dot{q}_{i}-\dot{q}_{j}\right)^{2},
$$

And the Euler-Lagrange equation is rewritten as:

$$
\frac{d}{d t}\left(\frac{\partial L}{\partial \dot{q}_{i}}\right)-\frac{\partial L}{\partial q_{i}}+\frac{\partial F}{\partial \dot{q}_{i}}=Q_{i} \quad, \quad i=1,2, \ldots, n
$$

\section{A.2.1. Kinetic Energy}

The kinetic energy of an extended mass is:

$$
T=\frac{1}{2} m\left(\dot{x}^{2}+\dot{y}^{2}\right)+\frac{1}{2} I \dot{\theta}^{2},
$$

where $\dot{x}$ is the velocity of the point along the $x$ direction and $\dot{y}$ is the velocity of the point along the y direction. The angular velocity of the extended mass around its center of mass is given by $\dot{\theta}$. The kinetic energy of multi-body systems can be written as the sum of the individual kinetic energies. 


$$
T=\sum_{i=1}^{n} T_{i}
$$

The masses can be connected together like multi-body systems or they can be individually connected to ground, and these connections lead to constraints, which reduce the number of DOF to the number of DOF of the system in the real world. The constraints are applied to the positions of the bodies. In this case the position of the $C G$ of segments will be:

$$
\begin{aligned}
r_{u} & =\left[C G_{u} \sin \left(\theta_{u}\right)+x_{0}\right] \cdot \hat{i}-\left[C G_{u} \cos \left(\theta_{u}\right)+y_{0}\right] \cdot \hat{j} \\
r_{f} & =\left[L_{u} \sin \left(\theta_{u}\right)+C G_{f} \sin \left(\theta_{u}+\theta_{f}\right)\right] \cdot \hat{i}-\left[L_{u} \cos \left(\theta_{u}\right)+C G_{f} \cos \left(\theta_{u}+\theta_{f}\right)\right] \cdot \hat{j}, \\
r_{h} & =\left[L_{u} \sin \left(\theta_{u}\right)+L_{f} \sin \left(\theta_{u}+\theta_{f}\right)\right] \cdot \hat{i}-\left[L_{u} \cos \left(\theta_{u}\right)+L_{f} \cos \left(\theta_{u}+\theta_{f}\right)\right] \cdot \hat{j}
\end{aligned}
$$

where $x_{0}$ and $y_{0}$ denote the positions of the reference frame at shoulder. It was assumed that $x_{0}=y_{0}=0$. Derivatives of the positions of points were taken with respect to time, in order to find the velocity:

$$
\begin{aligned}
\dot{r}_{u} & =\dot{\theta}_{u} C G_{u} \cos \left(\theta_{u}\right) \cdot \hat{i}+\dot{\theta}_{u} C G_{u} \sin \left(\theta_{u}\right) \cdot \hat{j} \\
r_{f} & =\left[\dot{\theta}_{u} L_{u} \cos \left(\theta_{u}\right)+\left(\dot{\theta}_{u}+\dot{\theta}_{f}\right) C G_{f} \cos \left(\theta_{u}+\theta_{f}\right)\right] \cdot \hat{i}+\left[\dot{\theta}_{f} L_{u} \sin \left(\theta_{u}\right)+\left(\dot{\theta}_{u}+\dot{\theta}_{f}\right) C G_{f} \sin \left(\theta_{u}+\theta_{f}\right)\right] \cdot \hat{j} \\
r_{h} & =\left[\dot{\theta}_{u} L_{u} \cos \left(\theta_{u}\right)+\left(\dot{\theta}_{u}+\dot{\theta}_{f}\right) L_{f} \cos \left(\theta_{u}+\theta_{f}\right)\right] \cdot \hat{i}+\left[\dot{\theta}_{f} L_{u} \sin \left(\theta_{u}\right)+\left(\dot{\theta}_{u}+\dot{\theta}_{f}\right) L_{f} \sin \left(\theta_{u}+\theta_{f}\right)\right] \cdot \hat{j}
\end{aligned}
$$

Thus the Kinetic energy of the upper extremities using (A. 7) will be:

$$
T=T_{u}+T_{f}+T_{h}=\left[\frac{1}{2} m_{u} v_{u}^{2}+\frac{1}{2} I_{u} \dot{\theta}_{u}^{2}\right]+\left[\frac{1}{2} m_{f} v_{f}^{2}+\frac{1}{2} I_{f}\left(\dot{\theta}_{f}+\dot{\theta}_{u}\right)^{2}\right]+\left[\frac{1}{2} m_{h} v_{h}^{2}\right] .
$$

Substituting (A. 9) in (A. 10): 


$$
\left\{\begin{array}{c}
T_{u}=\frac{1}{2} m_{u}\left(\left[\dot{\theta}_{u} C G_{u} \cos \left(\theta_{u}\right)\right]^{2}+\left[\dot{\theta}_{u} C G_{u} \sin \left(\theta_{u}\right)\right]^{2}\right)+\frac{1}{2} I_{u} \dot{\theta}_{u}^{2} \\
T_{f}=\frac{1}{2} m_{f}\left(\left[\dot{\theta}_{u} L_{u} \cos \left(\theta_{u}\right)+\left(\dot{\theta}_{u}+\dot{\theta}_{f}\right) C G_{f} \cos \left(\theta_{u}+\theta_{f}\right)\right]^{2}+\ldots\right. \\
\left.\ldots+\left[\dot{\theta}_{u} L_{u} \sin \left(\theta_{u}\right)+\left(\dot{\theta}_{u}+\dot{\theta}_{f}\right) C G_{f} \sin \left(\theta_{u}+\theta_{f}\right)\right]^{2}\right)+\frac{1}{2} I_{f}\left(\dot{\theta}_{f}+\dot{\theta}_{u}\right)^{2}, \\
T_{h}=\frac{1}{2} m_{h}\left(\left[\dot{\theta}_{u} L_{u} \cos \left(\theta_{u}\right)+\left(\dot{\theta}_{u}+\dot{\theta}_{f}\right) L_{f} \cos \left(\theta_{u}+\theta_{f}\right)\right]^{2}+\ldots\right. \\
\left.\ldots+\left[\dot{\theta}_{u} L_{u} \sin \left(\theta_{u}\right)+\left(\dot{\theta}_{u}+\dot{\theta}_{f}\right) L_{f} \sin \left(\theta_{u}+\theta_{f}\right)\right]^{2}\right)
\end{array}\right.
$$

Simplifying the equation and applying $\sin (\theta)^{2}+\cos (\theta)^{2}=1$, the following equation can be achieved.

$$
\left\{\begin{aligned}
T_{u}= & \frac{1}{2} \dot{\theta}_{u}{ }^{2} m_{u} C G_{u}{ }^{2}+\frac{1}{2} I_{u} \dot{\theta}_{u}^{2} \\
T_{f}= & \dot{\theta}_{u}^{2}\left[\frac{1}{2} m_{f} L_{u}^{2}\right]+\left(\dot{\theta}_{u}+\dot{\theta}_{f}\right)^{2}\left[\frac{1}{2} m_{f} C G_{f}^{2}\right]+m_{f} \dot{\theta}_{u}\left(\dot{\theta}_{u}+\dot{\theta}_{f}\right) L_{u} C G_{f}\left[\cos \left(\theta_{u}\right) \cos \left(\theta_{u}+\theta_{f}\right)+\sin \left(\theta_{u}\right) \sin \left(\theta_{u}+\theta_{f}\right)\right]+\ldots \\
& \ldots+\frac{1}{2} I_{f} \dot{\theta}_{f}{ }^{2}+\frac{1}{2} I_{f} \dot{\theta}_{u}^{2}+I_{f} \dot{\theta}_{f} \dot{\theta}_{u} \\
T_{h}= & \dot{\theta}_{u}{ }^{2}\left[\frac{1}{2} m_{h} L_{u}^{2}\right]+\left(\dot{\theta}_{u}+\dot{\theta}_{f}\right)^{2}\left[\frac{1}{2} m_{h} L_{f}^{2}\right]+m_{h} \dot{\theta}_{u}\left(\dot{\theta}_{u}+\dot{\theta}_{f}\right) L_{u} L_{f}\left[\cos \left(\theta_{u}\right) \cos \left(\theta_{u}+\theta_{f}\right)+\sin \left(\theta_{u}\right) \sin \left(\theta_{u}+\theta_{f}\right)\right]
\end{aligned}\right.
$$

Recalling equation (A. 7),

$$
\begin{aligned}
T= & \dot{\theta}_{u}^{2}\left[\frac{1}{2} m_{u} C G_{u}^{2}+\frac{1}{2} I_{u}+\frac{1}{2} m_{f} L_{u}^{2}+\frac{1}{2} m_{f} C G_{f}{ }^{2}+\frac{1}{2} I_{f}+\frac{1}{2} m_{h} L_{u}{ }^{2}+\frac{1}{2} m_{h} L_{f}{ }^{2}\right]+\ldots \\
& \ldots+\dot{\theta}_{f}^{2}\left[\frac{1}{2} m_{f} C G_{f}^{2}+\frac{1}{2} I_{f}+\frac{1}{2} m_{h} L_{f}{ }^{2}\right]+\ldots \\
& \ldots+\dot{\theta}_{u} \dot{\theta}_{f}\left[m_{f} C G_{f}{ }^{2}+I_{f}+m_{h} L_{f}{ }^{2}\right]+\ldots \\
& \ldots+\dot{\theta}_{u}\left(\dot{\theta}_{u}+\dot{\theta}_{f}\right)\left[\cos \left(\theta_{u}\right) \cos \left(\theta_{u}+\theta_{f}\right)+\sin \left(\theta_{u}\right) \sin \left(\theta_{u}+\theta_{f}\right)\right]\left[m_{f} L_{u} C G_{f}+m_{h} L_{u} L_{f}\right]
\end{aligned}
$$

Thus, the final equation will be

$$
T=M_{1} \cdot \dot{\theta}_{u}^{2}+M_{2} \cdot \dot{\theta}_{f}^{2}+M_{3} \cdot \dot{\theta}_{u} \dot{\theta}_{f}+M_{4} \cdot \dot{\theta}_{u}\left(\dot{\theta}_{u}+\dot{\theta}_{f}\right)\left[\cos \left(\theta_{u}\right) \cos \left(\theta_{u}+\theta_{f}\right)+\sin \left(\theta_{u}\right) \sin \left(\theta_{u}+\theta_{f}\right)\right]
$$

where 


$$
\begin{aligned}
& M_{1}=\frac{1}{2} m_{u} C G_{u}^{2}+\frac{1}{2} I_{u}+\frac{1}{2} m_{f} L_{u}^{2}+\frac{1}{2} m_{f} C G_{f}{ }^{2}+\frac{1}{2} I_{f}+\frac{1}{2} m_{h} L_{u}{ }^{2}+\frac{1}{2} m_{h} L_{f}{ }^{2}, \\
& M_{2}=\frac{1}{2} m_{f} C G_{f}{ }^{2}+\frac{1}{2} I_{f}+\frac{1}{2} m_{h} L_{f}^{2}, \\
& M_{3}=m_{f} C G_{f}{ }^{2}+I_{f}+m_{h} L_{f}{ }^{2}, \text { and } \\
& M_{4}=m_{f} L_{u} C G_{f}+m_{h} L_{u} L_{f}
\end{aligned}
$$

\section{A.2.2. Potential Energy}

Potential energy is the energy of an object or a system, which is gained or lost due to the position displacement. In our system there were two potential energies, gravitational and spring.

$$
U=\sum_{i=1}^{n} U_{G_{i}}+\sum_{i=1}^{n} U_{S_{i}} \quad, \quad i=1,2, \ldots, n,
$$

where $U_{G}$ denotes the gravitational potential energy and $U_{S}$ denotes spring potential energy.

$$
\left\{\begin{array}{l}
U_{G}=m_{u} g C G_{u}\left[1-\cos \left(\theta_{u}\right)\right]+m_{f} g\left[L_{u}\left(1-\cos \left(\theta_{u}\right)\right)+C G_{f}\left(1-\cos \left(\theta_{u}+\theta_{f}\right)\right)\right]+\ldots \\
\ldots+m_{h} g\left[L_{u}\left(1-\cos \left(\theta_{u}\right)\right)+L_{f}\left(1-\cos \left(\theta_{u}+\theta_{f}\right)\right)\right] \\
U_{S}=\frac{1}{2} k_{u}\left(\theta_{u}-\theta_{u 0}\right)^{2}+\frac{1}{2} k_{f}\left(\theta_{f}-\theta_{f 0}\right)^{2}
\end{array},\right.
$$

where $\theta_{u 0}$ denotes the initial angle of the shoulder joint. Putting equation (A. 14) in equation (A. 13) yields:

$$
\begin{aligned}
U= & m_{u} g C G_{u}\left[1-\cos \left(\theta_{u}\right)\right]+m_{f} g\left[L_{u}\left(1-\cos \left(\theta_{u}\right)\right)+C G_{f}\left(1-\cos \left(\theta_{u}+\theta_{f}\right)\right)\right]+\ldots \\
& \ldots+m_{h} g\left[L_{u}\left(1-\cos \left(\theta_{u}\right)\right)+L_{f}\left(1-\cos \left(\theta_{u}+\theta_{f}\right)\right)\right]+\frac{1}{2} k_{u}\left(\theta_{u}-\theta_{u 0}\right)^{2}+\frac{1}{2} k_{f}\left(\theta_{f}-\theta_{f 0}\right)^{2}
\end{aligned}
$$




\section{A.2.3. Rayleigh Dissipation Function}

Since two damping functions exist, one at the shoulder joint and the other one at the elbow joint, RDF must be included in the Euler-Lagrange equation:

$$
F=F_{u}+F_{f}=\frac{1}{2} c_{u}\left(\dot{\theta}_{u}-\dot{\theta}_{u 0}\right)^{2}+\frac{1}{2} c_{f}\left(\dot{\theta}_{f}-\dot{\theta}_{u}\right)^{2},
$$

where $\dot{\theta}_{u 0}$ is the velocity of the reference frame, which will be zero.

\section{A.2.4. Generalized Forces}

As for the generalized forces, there were two torques being applied on two joints. One is $T_{u}$, which was applied to the upper arm joint and the other one is $T_{f}$, which was applied to the forearm.

\section{A.2.5. Equation of Motion Using Euler-Lagrange Method and RDF}

Since there were two states in this system, $\theta_{u}$ and $\theta_{f}$, Euler-Lagrange method must be computed for both of them. Since the equations were lengthy, Euler-Lagrange equation were broken down into 4 parts, (a), (b), (c), and (d), and then compute them separately. Substituting (A. 1) in (A. 2) and rearranging the equation:

$$
\underbrace{\left[\frac{d}{d t}\left(\frac{\partial T}{\partial \dot{\theta}_{i}}\right)-\frac{\partial T}{\partial \theta_{i}}\right]}-\underbrace{\left[\frac{d}{d t}\left(\frac{\partial U}{\partial \dot{\theta}_{i}}\right)-\frac{\partial U}{\partial \theta_{i}}\right]} \underbrace{+\frac{\partial F}{\partial \dot{\theta}_{i}}}=Q_{i}, i=1,2, \ldots, n
$$

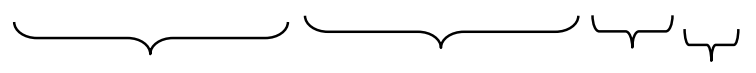

(a)

(b)

(c) (d)

There are four terms in this equation and each one will be derived accordingly. 


\section{A.2.5.1. Euler-Lagrange Equation for $\theta_{u}$}

\section{(a) Kinetic Energy}

Substituting (A. 12) in part (a) of (A. 17) and making the following simplifications yields:

$$
\frac{d}{d t}\left(\frac{\partial T}{\partial \dot{\theta}_{u}}\right)-\frac{\partial T}{\partial \theta_{u}}=2 M_{1} \ddot{\theta}_{u}+M_{3} \ddot{\theta}_{f}+M_{4}\left[2 \ddot{\theta}_{u}+\ddot{\theta}_{f}\right] \cdot\left[\cos \left(\theta_{u}\right) \cos \left(\theta_{u}+\theta_{f}\right)+\sin \left(\theta_{u}\right) \sin \left(\theta_{u}+\theta_{f}\right)\right]
$$

Recalling from trigonometry:

$$
\begin{aligned}
& \cos \left(\theta_{u}\right) \cos \left(\theta_{u}+\theta_{f}\right)+\sin \left(\theta_{u}\right) \sin \left(\theta_{u}+\theta_{f}\right)=\cos \left(-\theta_{f}\right)=\cos \left(\theta_{f}\right), \\
& \sin \left(\theta_{u}\right) \cos \left(\theta_{u}+\theta_{f}\right)-\cos \left(\theta_{u}\right) \sin \left(\theta_{u}+\theta_{f}\right)=\sin \left(-\theta_{f}\right)=-\sin \left(\theta_{f}\right),
\end{aligned}
$$

Thus the kinetic energy equation will be simplified further as bellow:

$$
\frac{d}{d t}\left(\frac{\partial T}{\partial \dot{\theta}_{u}}\right)-\frac{\partial T}{\partial \theta_{u}}=2 M_{1} \ddot{\theta}_{u}+M_{3} \ddot{\theta}_{f}+M_{4}\left[2 \ddot{\theta}_{u}+\ddot{\theta}_{f}\right] \cdot \cos \left(\theta_{f}\right),
$$

\section{(b) Potential Energy}

Substituting (A. 13) in part (b) of (A. 17) and making the following simplifications will yield:

$$
\begin{aligned}
\frac{d}{d t}\left(\frac{\partial U}{\partial \dot{\theta}_{i}}\right)-\frac{\partial U}{\partial \theta_{i}}= & \frac{d}{d t}[0]-m_{u} g C G_{u} \sin \left(\theta_{u}\right)+m_{f} g\left[L_{u} \sin \left(\theta_{u}\right)+C G_{f} \sin \left(\theta_{u}+\theta_{f}\right)\right]+\ldots \\
& \ldots+m_{h} g\left[L_{u} \sin \left(\theta_{u}\right)+L_{f} \sin \left(\theta_{u}+\theta_{f}\right)\right]+k_{u}\left(\theta_{u}-\theta_{u 0}\right) \\
= & 0-\left\{m_{u} g C G_{u} \sin \left(\theta_{u}\right)-m_{f} g\left[L_{u} \sin \left(\theta_{u}\right)+C G_{f} \sin \left(\theta_{u}+\theta_{f}\right)\right]-\ldots\right. \\
& \left.\ldots-m_{h} g\left[L_{u} \sin \left(\theta_{u}\right)+L_{f} \sin \left(\theta_{u}+\theta_{f}\right)\right]-k_{u}\left(\theta_{u}-\theta_{u 0}\right)\right\} \\
= & -m_{u} g C G_{u} \sin \left(\theta_{u}\right)-m_{f} g\left[L_{u} \sin \left(\theta_{u}\right)+C G_{f}\left[\sin \left(\theta_{u}\right) \cos \left(\theta_{f}\right)+\cos \left(\theta_{u}\right) \sin \left(\theta_{f}\right)\right]\right]-\ldots \\
& \ldots-m_{h} g\left[L_{u} \sin \left(\theta_{u}\right)+L_{f}\left[\sin \left(\theta_{u}\right) \cos \left(\theta_{f}\right)+\cos \left(\theta_{u}\right) \sin \left(\theta_{f}\right)\right]\right]-k_{u}\left(\theta_{u}-\theta_{u 0}\right)
\end{aligned}
$$

Thus the kinetic potential energy equation will be: 


$$
\begin{aligned}
\frac{d}{d t}\left(\frac{\partial U}{\partial \dot{\theta}_{i}}\right)-\frac{\partial U}{\partial \theta_{i}}= & \sin \left(\theta_{u}\right)\left(-m_{u} g C G_{u}-m_{f} g L_{u}-m_{h} g L_{u}\right)+\ldots \\
& \ldots+\left[\sin \left(\theta_{u}\right) \cos \left(\theta_{f}\right)+\cos \left(\theta_{u}\right) \sin \left(\theta_{f}\right)\right]\left(-m_{f} g C G_{f}-m_{h} g L_{f}\right)-k_{u}\left(\theta_{u}-\theta_{u 0}\right)
\end{aligned}
$$

\section{(c) Rayleigh Dissipation Function}

Substituting (A. 16) in part (c) of (A. 17) and making the following simplifications will yield:

$$
\frac{\partial F}{\partial \dot{\theta}_{u}}=c_{u}\left(\dot{\theta}_{u}-\dot{\theta}_{u 0}\right)-c_{f}\left(\dot{\theta}_{f}-\dot{\theta}_{u}\right),
$$

\section{(d) Summary}

Substituting (A. 20), (A. 21), and (A. 22) in (A. 17), and using (A. 18):

$$
\left\{\begin{array}{l}
\left\{\frac{d}{d t}\left(\frac{\partial T}{\partial \dot{\theta}_{u}}\right)-\frac{\partial T}{\partial \theta_{u}}\right\}+\left\{\frac{d}{d t}\left(\frac{\partial U}{\partial \dot{\theta}_{u}}\right)-\frac{\partial U}{\partial \theta_{u}}\right\}+\left\{\frac{\partial F}{\partial \dot{\theta}_{u}}\right\}=2 M_{1} \ddot{\theta}_{u}+M_{3} \ddot{\theta}_{f}+M_{4}\left[2 \ddot{\theta}_{u}+\ddot{\theta}_{f}\right] \cdot \cos \left(\theta_{f}\right)+\ldots \\
\ldots+\sin \left(\theta_{u}\right)\left(-m_{u} g C G_{u}-m_{f} g L_{u}-m_{h} g L_{u}\right)+\left[\sin \left(\theta_{u}\right) \cos \left(\theta_{f}\right)+\cos \left(\theta_{u}\right) \sin \left(\theta_{f}\right)\right]\left(-m_{f} g C G_{f}-m_{h} g L_{f}\right)-\ldots \\
\ldots-k_{u}\left(\theta_{u}-\theta_{u 0}\right)+c_{u}\left(\dot{\theta}_{u}-\dot{\theta}_{u 0}\right)-c_{f}\left(\dot{\theta}_{f}-\dot{\theta}_{u}\right) \quad=2 M_{1} \ddot{\theta}_{u}+M_{3} \ddot{\theta}_{f}+M_{4}\left[2 \ddot{\theta}_{u}+\ddot{\theta}_{f}\right] \cdot \cos \left(\theta_{f}\right)+\ldots \\
\ldots+M_{5} \sin \left(\theta_{u}\right)+M_{6}\left[\sin \left(\theta_{u}\right) \cos \left(\theta_{f}\right)+\cos \left(\theta_{u}\right) \sin \left(\theta_{f}\right)\right]-k_{u}\left(\theta_{u}-\theta_{u 0}\right)+c_{u}\left(\dot{\theta}_{u}-\dot{\theta}_{u 0}\right)-c_{f}\left(\dot{\theta}_{f}-\dot{\theta}_{u}\right)
\end{array}\right.
$$

where $M_{1}$ to $M_{4}$ were defined in

(A. 12) and $M_{5}$ to $M_{6}$ are:

$$
\begin{aligned}
& M_{5}=-m_{u} g C G_{u}-m_{f} g L_{u}-m_{h} g L_{u}, \\
& M_{6}=-m_{f} g C G_{f}-m_{h} g L_{f}
\end{aligned}
$$

Rearranging the equation, the Euler-Lagrange equation for $\theta_{u}$ will be:

$$
\begin{aligned}
\left\{\frac{d}{d t}\left(\frac{\partial T}{\partial \dot{\theta}_{u}}\right)-\frac{\partial T}{\partial \theta_{u}}\right\}+\left\{\frac{d}{d t}\left(\frac{\partial U}{\partial \dot{\theta}_{u}}\right)-\frac{\partial U}{\partial \theta_{u}}\right\}+\left\{\frac{\partial F}{\partial \dot{\theta}_{u}}\right\}=\left\{2 M_{1} \ddot{\theta}_{u}+M_{3} \ddot{\theta}_{f}\right\}+\ldots \\
\ldots+ \\
\left.\ldots+\dot{\theta}_{u}\left[c_{u}+c_{f}\right]+c_{u}\left(-\dot{\theta}_{u 0}\right)-k_{u}\left(\theta_{u}\right)+k_{u}\left(\theta_{u 0}\right)-\dot{\theta}_{f} c_{f}\right\}+\ldots \\
\ldots+M_{4}\left[2 \ddot{\theta}_{u}+\ddot{\theta}_{f}\right] \cdot \cos \left(\theta_{f}\right)+M_{5} \sin \left(\theta_{u}\right)+M_{6}\left[\sin \left(\theta_{u}\right) \cos \left(\theta_{f}\right)+\cos \left(\theta_{u}\right) \sin \left(\theta_{f}\right)\right]
\end{aligned}
$$

where the definitions of the moment constants are provided here again for convenience: 


$$
\begin{aligned}
& M_{1}=\frac{1}{2} m_{u} C G_{u}^{2}+\frac{1}{2} I_{u}+\frac{1}{2} m_{f} L_{u}^{2}+\frac{1}{2} m_{f} C G_{f}{ }^{2}+\frac{1}{2} I_{f}+\frac{1}{2} m_{h} L_{u}^{2}+\frac{1}{2} m_{h} L_{f}^{2} \\
& M_{3}=m_{f} C G_{f}{ }^{2}+I_{f}+m_{h} L_{f}^{2} \\
& M_{4}=m_{f} L_{u} C G_{f}+m_{h} L_{u} L_{f} \\
& M_{5}=-m_{u} g C G_{u}-m_{f} g L_{u}-m_{h} g L_{u} \\
& M_{6}=-m_{f} g C G_{f}-m_{h} g L_{f}
\end{aligned}
$$

\section{A.2.5.2. Euler-Lagrange Equation for $\theta_{f}$}

\section{(a) Kinetic Energy}

Substituting (A. 12) in part (a) of (A. 17) and making the following simplifications:

$$
\begin{aligned}
\frac{d}{d t}\left(\frac{\partial T}{\partial \dot{\theta}_{f}}\right)-\frac{\partial T}{\partial \theta_{f}}= & \frac{d}{d t}\left\{2 M_{2} \cdot \dot{\theta}_{f}+M_{3} \cdot \dot{\theta}_{u}+M_{4} \cdot \dot{\theta}_{u}\left[\cos \left(\theta_{u}\right) \cos \left(\theta_{u}+\theta_{f}\right)+\sin \left(\theta_{u}\right) \sin \left(\theta_{u}+\theta_{f}\right)\right]\right\}-\ldots \\
& \ldots-M_{4} \cdot \dot{\theta}_{u}\left(\dot{\theta}_{u}+\dot{\theta}_{f}\right)\left[-\cos \left(\theta_{u}\right) \sin \left(\theta_{u}+\theta_{f}\right)+\sin \left(\theta_{u}\right) \cos \left(\theta_{u}+\theta_{f}\right)\right]
\end{aligned}
$$

Using (A. 18) and (A. 19):

$$
\begin{aligned}
\frac{d}{d t}\left(\frac{\partial T}{\partial \dot{\theta}_{f}}\right)-\frac{\partial T}{\partial \theta_{f}} & =\frac{d}{d t}\left\{2 M_{2} \cdot \dot{\theta}_{f}+M_{3} \cdot \dot{\theta}_{u}+M_{4} \cdot \dot{\theta}_{u}\left[\cos \left(\theta_{f}\right)\right]\right\}-M_{4} \cdot \dot{\theta}_{u}\left(\dot{\theta}_{u}+\dot{\theta}_{f}\right)\left[-\sin \left(\theta_{f}\right)\right] \\
& =\frac{d}{d t}\left\{2 M_{2} \cdot \dot{\theta}_{f}+M_{3} \cdot \dot{\theta}_{u}+M_{4} \cdot \dot{\theta}_{u} \cos \left(\theta_{f}\right)\right\}+M_{4} \cdot \dot{\theta}_{u}\left(\dot{\theta}_{u}+\dot{\theta}_{f}\right) \sin \left(\theta_{f}\right)
\end{aligned}
$$

Thus the kinetic energy equation will be:

$$
\frac{d}{d t}\left(\frac{\partial T}{\partial \dot{\theta}_{f}}\right)-\frac{\partial T}{\partial \theta_{f}}=2 M_{2} \cdot \ddot{\theta}_{f}+M_{3} \cdot \ddot{\theta}_{u}+M_{4} \cdot \ddot{\theta}_{u} \cos \left(\theta_{f}\right)-M_{4} \cdot \dot{\theta}_{u} \sin \left(\theta_{f}\right)+M_{4} \cdot \dot{\theta}_{u}\left(\dot{\theta}_{u}+\dot{\theta}_{f}\right) \sin \left(\theta_{f}\right)
$$

\section{(b) Potential Energy}

Substituting (A. 15) in part (b) of (A. 17) and doing the following simplifications: 


$$
\begin{aligned}
\frac{d}{d t}\left(\frac{\partial U}{\partial \dot{\theta}_{f}}\right)-\frac{\partial U}{\partial \theta_{f}} & =\frac{d}{d t}\{0\}-\left[m_{f} g C G_{f} \sin \left(\theta_{u}+\theta_{f}\right)+m_{h} g L_{f} \sin \left(\theta_{u}+\theta_{f}\right)+k_{u}\left(\theta_{f}-\theta_{f 0}\right)\right] \\
& =-m_{f} g C G_{f} \sin \left(\theta_{u}+\theta_{f}\right)-m_{h} g L_{f} \sin \left(\theta_{u}+\theta_{f}\right)-k_{u}\left(\theta_{f}-\theta_{f 0}\right)
\end{aligned}
$$

\section{(c) Rayleigh Dissipation Function}

Substituting (A. 16) in part (c) of (A. 17) and doing the following simplifications:

$$
\frac{\partial F}{\partial \dot{\theta}_{f}}=c_{f}\left(\dot{\theta}_{f}-\dot{\theta}_{u}\right)
$$

\section{(d) Summary}

Substituting (A. 25), (A. 26), and (A. 27) in (A. 17):

$$
\begin{gathered}
\left\{\frac{d}{d t}\left(\frac{\partial T}{\partial \dot{\theta}_{f}}\right)-\frac{\partial T}{\partial \theta_{f}}\right\}+\left\{\frac{d}{d t}\left(\frac{\partial U}{\partial \dot{\theta}_{f}}\right)-\frac{\partial U}{\partial \theta_{f}}\right\}+\left\{\frac{\partial F}{\partial \dot{\theta}_{f}}\right\}=2 M_{2} \cdot \ddot{\theta}_{f}+M_{3} \cdot \ddot{\theta}_{u}+M_{4} \cdot \ddot{\theta}_{u} \cos \left(\theta_{f}\right)-\ldots \\
\quad \ldots-M_{4} \cdot \dot{\theta}_{u} \sin \left(\theta_{f}\right)+M_{4} \cdot \dot{\theta}_{u}\left(\dot{\theta}_{u}+\dot{\theta}_{f}\right) \sin \left(\theta_{f}\right)-m_{f} g C G_{f} \sin \left(\theta_{u}+\theta_{f}\right)-m_{h} g L_{f} \sin \left(\theta_{u}+\theta_{f}\right)-\ldots \\
\quad \ldots-k_{u}\left(\theta_{f}-\theta_{f 0}\right)+c_{f}\left(\dot{\theta}_{f}-\dot{\theta}_{u}\right)
\end{gathered}
$$

Simplifying this function, the following function was obtained:

$$
\begin{aligned}
& \left\{\frac{d}{d t}\left(\frac{\partial T}{\partial \dot{\theta}_{f}}\right)-\frac{\partial T}{\partial \theta_{f}}\right\}+\left\{\frac{d}{d t}\left(\frac{\partial U}{\partial \dot{\theta}_{f}}\right)-\frac{\partial U}{\partial \theta_{f}}\right\}+\left\{\frac{\partial F}{\partial \dot{\theta}_{f}}\right\}=\left[2 M_{2} \cdot \ddot{\theta}_{f}+M_{3} \cdot \ddot{\theta}_{u}\right]+\left[c_{f}\left(\dot{\theta}_{f}-\dot{\theta}_{u}\right)\right]-\ldots \\
& \ldots-\left[k_{u}\left(\theta_{f}-\theta_{f 0}\right)\right]+\left[M_{4} \cdot \ddot{\theta}_{u} \cos \left(\theta_{f}\right)-M_{4} \cdot \dot{\theta}_{u} \sin \left(\theta_{f}\right)+M_{4} \cdot \dot{\theta}_{u}\left(\dot{\theta}_{u}+\dot{\theta}_{f}\right) \sin \left(\theta_{f}\right)+M_{7} \sin \left(\theta_{u}+\theta_{f}\right)\right]
\end{aligned}
$$

where

$$
\begin{array}{ll}
M_{2}=\frac{1}{2} m_{f} C G_{f}^{2}+\frac{1}{2} I_{f}+\frac{1}{2} m_{h} L_{f}^{2} & M_{3}=m_{f} C G_{f}^{2}+I_{f}+m_{h} L_{f}^{2} \\
M_{4}=m_{f} L_{u} C G_{f}+m_{h} L_{u} L_{f} & M_{7}=-m_{f} g C G_{f}-m_{h} g L_{f}
\end{array}
$$




\section{A.3. Forming Equations of Motion}

Placing (A. 23) and (A. 28) in (A. 5) and considering the external torque $T_{u}$ and $T_{l}$, and (A. 24) and (A. 29) the equation will be:

$\left\{\begin{array}{l}\text { 1) }\left\{2 M_{1} \ddot{\theta}_{u}+M_{3} \ddot{\theta}_{f}\right\}+\left\{\dot{\theta}_{u}\left[c_{u}+c_{f}\right]+c_{u}\left(-\dot{\theta}_{u 0}\right)-k_{u}\left(\theta_{u}\right)+k_{u}\left(\theta_{u 0}\right)-\dot{\theta}_{f} c_{f}\right\}+\ldots \\ \ldots+\left\{M_{4}\left[2 \ddot{\theta}_{u}+\ddot{\theta}_{f}\right] \cdot \cos \left(\theta_{f}\right)+M_{5} \sin \left(\theta_{u}\right)+M_{6} \sin \left(\theta_{u}+\theta_{f}\right)\right\}=T_{u} \\ \text { 2) }\left\{2 M_{2} \cdot \ddot{\theta}_{f}+M_{3} \cdot \ddot{\theta}_{u}\right\}+\left\{c_{f}\left(\dot{\theta}_{f}-\dot{\theta}_{u}\right)\right\}-\left\{k_{u}\left(\theta_{f}-\theta_{f 0}\right)\right\}+\ldots \\ \quad \ldots+\left\{M_{4} \cdot \ddot{\theta}_{u} \cos \left(\theta_{f}\right)-M_{4} \cdot \dot{\theta}_{u} \sin \left(\theta_{f}\right)+M_{4} \cdot \dot{\theta}_{u}\left(\dot{\theta}_{u}+\dot{\theta}_{f}\right) \sin \left(\theta_{f}\right)+M_{7} \sin \left(\theta_{u}+\theta_{f}\right)\right\}=T_{f}\end{array}\right.$

where

$$
\left\{\begin{array}{l}
M_{1}=\frac{1}{2} m_{u} C G_{u}^{2}+\frac{1}{2} I_{u}+\frac{1}{2} m_{f} L_{u}^{2}+\frac{1}{2} m_{f} C G_{f}^{2}+\frac{1}{2} I_{f}+\frac{1}{2} m_{h} L_{u}^{2}+\frac{1}{2} m_{h} L_{f}^{2} \\
M_{2}=\frac{1}{2} m_{f} C G_{f}^{2}+\frac{1}{2} I_{f}+\frac{1}{2} m_{h} L_{f}^{2} \\
M_{3}=m_{f} C G_{f}^{2}+I_{f}+m_{h} L_{f}^{2} \\
M_{4}=m_{f} L_{u} C G_{f}+m_{h} L_{u} L_{f} \\
M_{5}=-m_{u} g C G_{u}-m_{f} g L_{u}-m_{h} g L_{u} \\
M_{6}=-m_{f} g C G_{f}-m_{h} g L_{f} \\
M_{7}=-m_{f} g C G_{f}-m_{h} g L_{f}
\end{array}\right.
$$




\section{Appendix B: Kinematics Modeling}

\section{B.1. Introduction}

In this appendix a Kinematic equations of motion of the upper extremities for 2-D space will be presented. This model will be used in order to evaluate position, velocity, and acceleration of a multi-body system (human limbs), while doing an ADL. The results of this chapter will be used as the states of appendix a to predict the required torque for each joint.

\section{B.2. Kinematics of the Upper Extremities [222]}

The system is modeled in a planar coordinate frame. The position and orientation of bodies in planar motion can be easily defined by two position coordinates and one rotation coordinate. Both absolute constraints of the motion of a single body and relative constraints of motion of several bodies will be formulated according to these Cartesian generalized coordinates. 


\section{Kinematic Analysis in Planar Cartesian Coordinates}

Kinematics is the study of motion including position, velocity and acceleration. Kinematic analysis is necessary to determine the dynamic response of a system once a force is applied. All equations derived in this section incorporate the rigid body assumption, i.e. there is no bending of the individual segments.

A rigid body is defined as a system of particles in which the distances between them remain unchanged. The concept of rigid body is not absolutely true in reality since any entity deforms once a force is applied. However, the concept is acceptable if the deformation is small compared with the overall movement.

Each rigid body is characterized by an attached position vector moving with the body called a body-fixed reference frame. All bodies in a system are characterized by position vectors associated to each one in a global reference frame. In other words, the configuration of a system is defined by a set of variables, called generalized coordinates, which uniquely specify the position and orientation of all bodies in a system. Generalized coordinates may be independent or dependent as required to satisfy the constraints. It should be noted that equations (B. 1) to (B. 10) were entirely taken from Haug [222]. The generalized coordinates of a system in motion are time variant and are denoted by a column vector as

$$
\mathbf{q}=\left[q_{1}, q_{2}, \ldots, q_{n}\right]^{T}
$$

To specify the planar position of each body, a body fixed $x_{i}-y_{i}$ frame, where $i=1,2, \ldots, n$ is attached to each body. The angle $\phi_{i}$ is the angle of rotation of this frame relative to the global $x_{0}$ -

$y_{0}$. Thus, the column vector $\mathbf{q}_{i}=\left[x_{1}, y_{2}, \phi_{n}\right]^{T}$ is the vector of planar Cartesian generalized 
coordinates for body $i$. The generalized coordinates of bodies are related to each other through equations of constraints. Thus Cartesian generalized coordinates of a system are generally dependent.

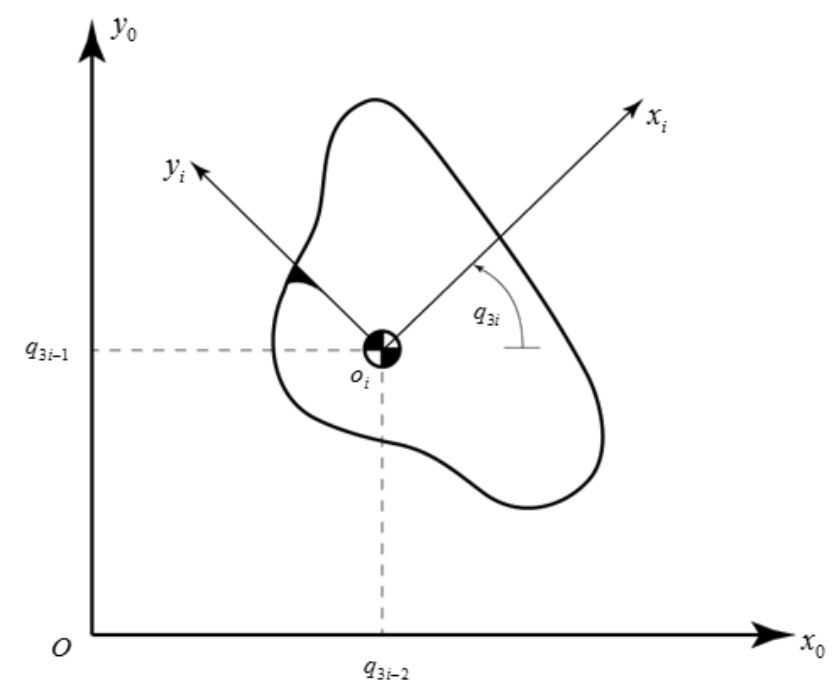

Figure B. 1. Position and orientation of body-fixed reference frame relative to global reference frame

The conditions being imposed by the kinematic constraints between two bodies relate the motion of one to the other. These conditions are expressed as algebraic equation in terms of generalized coordinates, which are called holonomic kinematic constraint equations. Holonomic constraints are the relation between the coordinates (time may be included) of a system, which can be expressed as

$$
f\left(q_{1}, q_{2}, \ldots, q_{n}, t\right)=0,
$$

Velocity or acceleration-dependent constraint such as $f\left(q_{1}, q_{2}, \ldots, q_{n}, \dot{q}_{1}, \dot{q}_{2}, \ldots, \dot{q}_{n}, t\right)=0$ are not holonomic. The general format of a system of $n$ holonomic kinematic constraint equations that explicitly depends on time can be expressed as

$$
\Phi^{K}(\mathbf{q}, t)=0,
$$




$$
\Phi^{K}(\mathbf{q}, t)=\left[\Phi_{1}{ }^{K}(\mathbf{q}, t), \Phi_{2}{ }^{K}(\mathbf{q}, t), \ldots, \Phi_{n h}{ }^{K}(\mathbf{q}, t)\right],
$$

where $t$ is time and $n h$ is the number of kinematic constraint equations. If the equation is non timedependent, it is called a stationary constraint. This is very crucial since the equation of constraint is obtained from the geometry of the system. Each kinematic constraint equation has a physical sense, which should be represented correctly by algebraic equations.

If a mechanical system allows its components to have motion, the number of generalized coordinates used to describe a system $(n c)$ will be greater than the number of holonomic kinematic equations $(n h)$. Degrees of freedom (DOF) is defined as $n c-n h$. The motion of a system is the resultant of (1) driving constraints (kinematic analysis) or (2) forces applied on the system (dynamic analysis).

This section is dedicated to the kinematic analysis of the robotic system and the effect of driving constraints on the motion of the mechanical system, the driving constraints equation is defined as

$$
\Phi^{D}(\mathbf{q}, t)=0,
$$

The configuration of the combined constraint equation would be

$$
\boldsymbol{\Phi}(\mathbf{q}, t)=\left[\begin{array}{l}
\boldsymbol{\Phi}^{K}(\mathbf{q}, t) \\
\boldsymbol{\Phi}^{D}(\mathbf{q}, t)
\end{array}\right]_{(n d+n c) \times 1},
$$

where $n d$ is the number of driving constraints. To acquire the velocity and acceleration of $\mathbf{q}$, the chain rule of differentiation is applied to (B. 4) as

$$
\Phi=\Phi_{\mathbf{q}} \dot{\mathbf{q}}+\Phi_{t}=0,
$$

In order to evaluate the velocity, $\dot{\mathbf{q}}$, in the following equation must be solved

$$
\Phi_{q} \dot{q}=-\Phi_{t} \equiv \mathbf{v},
$$


where $\Phi_{\mathrm{q}}$ is called the Jacobian matrix. $\Phi_{\mathrm{q}}$ and $\Phi_{t}$ are defined as

$$
\begin{gathered}
\boldsymbol{\Phi}_{\mathbf{q}}=\left[\frac{\partial \Phi_{i}}{\partial q_{i}}\right]_{n c \times n h}, \\
\boldsymbol{\Phi}_{t}=\left[\frac{\partial \Phi_{i}}{\partial t}\right]_{n c \times n h},
\end{gathered}
$$

Applying the chain rule of differentiation to (B. 5)

$$
\Phi_{\mathbf{q}} \ddot{\mathbf{q}}+\left(\Phi_{\mathbf{q}} \dot{\mathbf{q}}\right)_{\mathbf{q}} \dot{\mathbf{q}}+\Phi_{\mathbf{q} t} \dot{\mathbf{q}}=-\Phi_{t \mathbf{q}} \dot{\mathbf{q}}-\Phi_{t t}
$$

Since $\Phi_{t \mathrm{q}}=\Phi_{\mathrm{q} t}$, the equation can be rewritten as in (B. 9). The solution of the following is used to evaluate $\ddot{\mathbf{q}}$.

$$
\Phi_{\mathbf{q}} \ddot{\mathbf{q}}=-\left(\Phi_{\mathbf{q}} \dot{\mathbf{q}}\right)_{\mathbf{q}} \dot{\mathbf{q}}-2 \Phi_{\mathbf{q}} \dot{\mathbf{q}}-\Phi_{t t}
$$

where

$$
\begin{gathered}
\boldsymbol{\Phi}_{t t}=\left[\frac{\partial^{2} \boldsymbol{\Phi}_{i}}{\partial t^{2}}\right]_{n h \times 1}, \\
\boldsymbol{\Phi}_{\mathbf{q} t}=\left[\frac{\partial^{2} \boldsymbol{\Phi}_{i}}{\partial q_{j} \partial t}\right]_{n h \times n c}, \\
\left(\boldsymbol{\Phi}_{\mathbf{q}} \dot{\mathbf{q}}\right)_{\mathbf{q}}=\left[\frac{\partial}{\partial q_{j}}\left(\sum_{k=1}^{n c} \frac{\partial \Phi_{i}}{\partial q_{k}} \dot{q}_{k}\right)\right]_{n c \times n c}=\left[\sum_{k=1}^{n c} \frac{\partial^{2} \boldsymbol{\Phi}_{i}}{\partial q_{j} \partial q_{k}} \dot{q}_{k}\right]_{n c \times n c},
\end{gathered}
$$

If the number of constraints (including driving and kinematic constraints) is less than the number of holonomic kinematic equations $(n c+n d<n h)$, then there are some DOFs, which are not driven. Thus, the Jacobian matrix is non-square and consequently non-invertible. As a result, it is 
not possible to acquire velocity and acceleration through inverting the Jacobian matrix. Generalized inverse or Pseudoinverse method is one of the various numerical methods to obtain the inverse of a non-square matrix, which is used in this text too.

\section{Kinematic Modeling of the Upper Extremities}

To model the kinematic of the mechanical system, the generalized coordinates need to be defined first. Since the system can be defined in a planar space, each body can be defined by two translations and one rotation. Thus, the generalized coordinates are defined as

$$
\mathbf{q}=\left[q_{1}, q_{2}, q_{3}, q_{4}, q_{5}, q_{6}\right]^{T}
$$

where $q_{1}, q_{2}, q_{4}$, and $q_{5}$ are translational variables and $q_{3}$ and $q_{6}$ are rotational variables as shown in . Generalized coordinates of the robotic system.

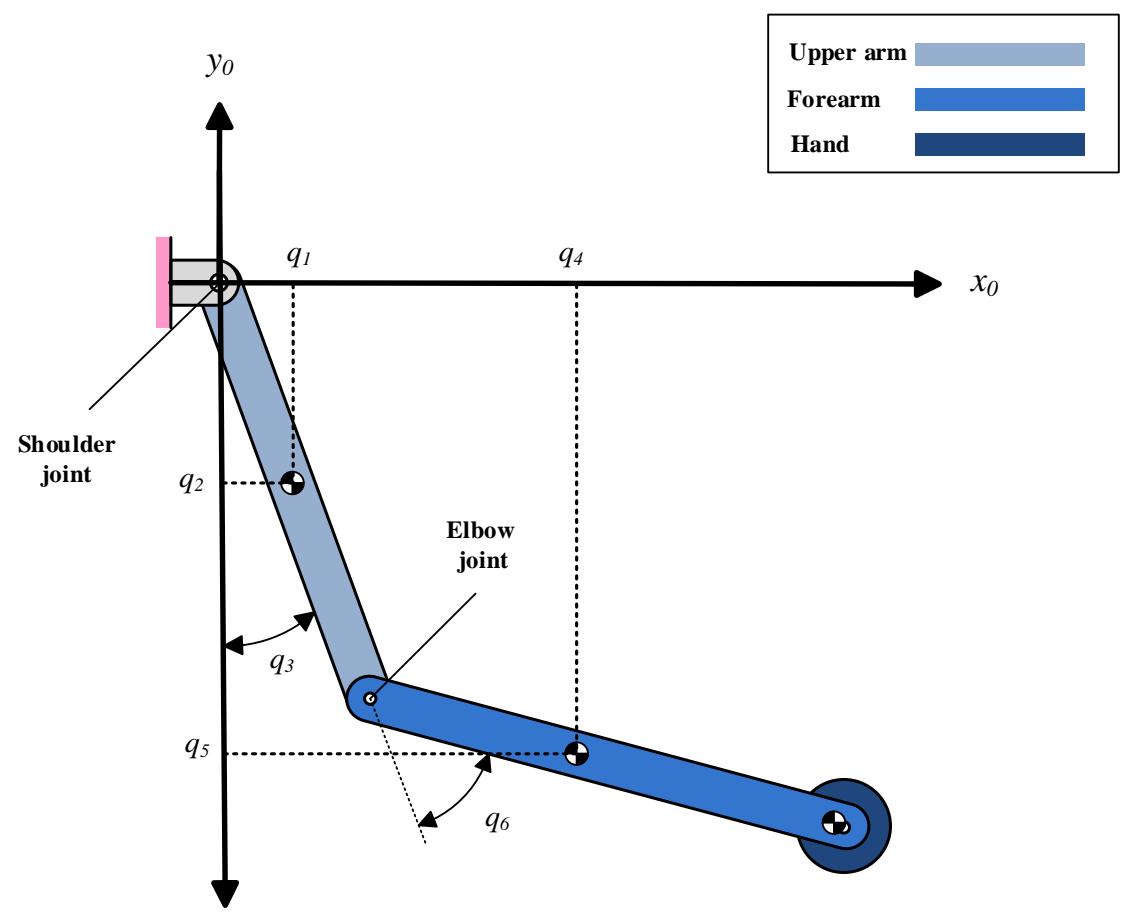

Figure B. 2. Generalized coordinates of the robotic system 
In order to form the constraint equations, the physical properties, which connect the segments (kinematic constraints) and driving constraints need to be identified. There are two joints and each one consists of three coordinates, which only two of them are kinematic constraints and the remaining one is a driving constraint. Thus the kinematic and driving constraint equations will be:

$$
\boldsymbol{\Phi}^{K}(\mathbf{q}, t)=\left[\begin{array}{c}
q_{1}-C G_{u} \sin \left(q_{3}\right) \\
q_{2}+C G_{u} \cos \left(q_{3}\right) \\
q_{1}+\left(L_{u}-C G_{u}\right) \sin \left(q_{3}\right)+C G_{f} \sin \left(q_{3}+q_{6}\right)-q_{4} \\
q_{2}-\left(L_{u}-C G_{u}\right) \cos \left(q_{3}\right)-C G_{f} \cos \left(q_{3}+q_{6}\right)-q_{5}
\end{array}\right]=0,
$$

where $C G_{u}, C G_{f}$, and $L_{u}$ are constants and are defined as the distance of the center of mass of the upper arm from proximal (closest joint to body), the distance of the center of mass of the forearm from proximal, and the length of the upper arm respectively and

$$
\boldsymbol{\Phi}^{D}(\mathbf{q}, t)=\left[\begin{array}{c}
q_{3}-q_{03}-\omega_{u} t \\
q_{6}-q_{06}-\omega_{f} t
\end{array}\right]=0,
$$

where $q_{03}$ and $q_{0} 6$ are the initial value for $q_{3}$ and $q_{6}$; and $\omega_{u}$ and $\omega_{f}$ are the angular velocity of the shoulder and elbow joints respectively. Thus the combined constraint equation based on (B. 4) would be

$$
\boldsymbol{\Phi}^{K}(\mathbf{q}, t)=\left[\begin{array}{c}
q_{1}-C G_{u} \sin \left(q_{3}\right) \\
q_{2}+C G_{u} \cos \left(q_{3}\right) \\
q_{1}+\left(L_{u}-C G_{u}\right) \sin \left(q_{3}\right)+C G_{f} \sin \left(q_{3}+q_{6}\right)-q_{4} \\
q_{2}-\left(L_{u}-C G_{u}\right) \cos \left(q_{3}\right)-C G_{f} \cos \left(q_{3}+q_{6}\right)-q_{5} \\
q_{3}-q_{03}-\omega_{u} t \\
q_{6}-q_{06}-\omega_{f} t
\end{array}\right],
$$

In order to obtain velocity through the solution of (B. 6), $\Phi_{\mathrm{q}}$ and $\Phi_{t}$ need to be calculated

first. Using (B. 7), the Jacobian matric $\Phi_{\mathrm{q}}$ and $\Phi_{t}$ would be 


$$
\begin{aligned}
\Phi_{\mathbf{q}} & =\left[\frac{\partial \Phi_{i}}{\partial q_{i}}\right]_{n c \times n h} \\
& =\left[\begin{array}{cccccc}
1 & 0 & -C G_{u} \cos \left(q_{3}\right) & 0 & 0 & 0 \\
0 & 1 & -C G_{u} \sin \left(q_{3}\right) & 0 & 0 & 0 \\
1 & 0 & \left(L_{u}-C G_{u}\right) \cos \left(q_{3}\right)+C G_{f} \cos \left(q_{3}+q_{6}\right) & -1 & 0 & C G_{f} \cos \left(q_{3}+q_{6}\right) \\
0 & 1 & \left(L_{u}-C G_{u}\right) \sin \left(q_{3}\right)+C G_{f} \sin \left(q_{3}+q_{6}\right) & 0 & -1 & C G_{f} \sin \left(q_{3}+q_{6}\right) \\
0 & 0 & 1 & 0 & 0 & 0 \\
0 & 0 & 0 & 0 & 0 & 1
\end{array}\right]
\end{aligned}
$$

$$
\boldsymbol{\Phi}_{t}=\left[\frac{\partial \Phi_{i}}{\partial t}\right]_{n c \times n h}=\left[\begin{array}{c}
0 \\
0 \\
0 \\
0 \\
-\omega_{u} \\
-\omega_{f}
\end{array}\right],
$$

Knowing $\Phi_{q}$ and $\Phi_{t}$, the velocity vector would be the solution of (B. 6).

To obtain the acceleration through (B. 9), $\left(\Phi_{\mathbf{q}} \dot{\mathbf{q}}\right)_{\mathbf{q}}, \Phi_{q \mathrm{q}}$, and $\boldsymbol{\Phi}_{t t}$ must be obtained. Using (B.

10) the two vectors are

$$
\begin{aligned}
& \boldsymbol{\Phi}_{t t}=\left[\frac{\partial^{2} \boldsymbol{\Phi}_{i}}{\partial t^{2}}\right]_{n h \times 1}=[0]_{n h \times 1}, \\
& \boldsymbol{\Phi}_{\mathbf{q}}=\left[\frac{\partial^{2} \boldsymbol{\Phi}_{i}}{\partial q_{j} \partial t}\right]_{n h \times n c}=[0]_{n h \times n c},
\end{aligned}
$$

Obtaining $\left(\Phi_{\mathbf{q}} \dot{\mathbf{q}}\right)_{\mathbf{q}}$, requires obtaining $\Phi_{q} \dot{\mathbf{q}}$ first. $\Phi_{q} \dot{\boldsymbol{q}}$ is obtained as 


$$
\Phi_{\mathbf{q}} \dot{\mathbf{q}}=\left[\begin{array}{c}
\dot{q}_{1}-\dot{q}_{3} C G_{u} \cos \left(q_{3}\right) \\
\dot{q}_{2}-\dot{q}_{3} C G_{u} \sin \left(q_{3}\right) \\
\dot{q}_{1}+\dot{q}_{3}\left(L_{u}-C G_{u}\right) \cos \left(q_{3}\right)+\dot{q}_{3} C G_{f} \cos \left(q_{3}+q_{6}\right)-\dot{q}_{4}+\dot{q}_{6} C G_{f} \cos \left(q_{3}+q_{6}\right) \\
\dot{q}_{2}+\dot{q}_{3}\left(L_{u}-C G_{u}\right) \sin \left(q_{3}\right)+\dot{q}_{3} C G_{f} \sin \left(q_{3}+q_{6}\right)-\dot{q}_{5}+\dot{q}_{6} C G_{f} \sin \left(q_{3}+q_{6}\right) \\
\dot{q}_{3} \\
\dot{q}_{6}
\end{array}\right],
$$

Thus $\left(\boldsymbol{\Phi}_{\mathbf{q}} \dot{\mathbf{q}}\right)_{\mathbf{q}}$ would be as

$$
\left(\Phi_{\mathbf{q}} \dot{\mathbf{q}}\right)_{\mathbf{q}}=\left[\begin{array}{c}
\dot{q}_{3} C G_{u} \sin \left(q_{3}\right) \\
-\dot{q}_{3} C G_{u} \cos \left(q_{3}\right) \\
-\dot{q}_{3}\left(L_{u}-C G_{u}\right) \sin \left(q_{3}\right)-2 \dot{q}_{3} C G_{f} \sin \left(q_{3}+q_{6}\right)-2 \dot{q}_{6} C G_{f} \sin \left(q_{3}+q_{6}\right) \\
\dot{q}_{3}\left(L_{u}-C G_{u}\right) \cos \left(q_{3}\right)+2 \dot{q}_{3} C G_{f} \cos \left(q_{3}+q_{6}\right)+2 \dot{q}_{6} C G_{f} \cos \left(q_{3}+q_{6}\right) \\
0 \\
0
\end{array}\right],
$$

Subsequently $\left(\boldsymbol{\Phi}_{\mathbf{q}} \mathbf{q}\right)_{\mathbf{q}} \mathbf{q}$ is obtained as

$$
\left(\Phi_{\mathbf{q}} \dot{\mathbf{q}}\right)_{\mathbf{q}} \dot{\mathbf{q}}=\left[\begin{array}{c}
\dot{q}_{3}^{2} C G_{u} \sin \left(q_{3}\right) \\
-\dot{q}_{3}^{2} C G_{u} \cos \left(q_{3}\right) \\
-\dot{q}_{3}^{2}\left(L_{u}-C G_{u}\right) \sin \left(q_{3}\right)-2 \dot{q}_{3}^{2} C G_{f} \sin \left(q_{3}+q_{6}\right)-2 \dot{q}_{6}^{2} C G_{f} \sin \left(q_{3}+q_{6}\right) \\
\dot{q}_{3}^{2}\left(L_{u}-C G_{u}\right) \cos \left(q_{3}\right)+2 \dot{q}_{3}^{2} C G_{f} \cos \left(q_{3}+q_{6}\right)+2 \dot{q}_{6}^{2} C G_{f} \cos \left(q_{3}+q_{6}\right) \\
0 \\
0
\end{array}\right],
$$

Since $\boldsymbol{\Phi}_{t t}$ and $\boldsymbol{\Phi}_{\mathbf{q} t}$ are zero matrices, (B. 9) will be simplified as

$$
\Phi_{\mathrm{q}} \mathrm{q}=-\left(\Phi_{\mathrm{q}} \mathrm{q}\right)_{\mathrm{q}} \mathrm{q} \stackrel{\text { def }}{=} \gamma
$$




\section{Appendix C: Technical Drawing}




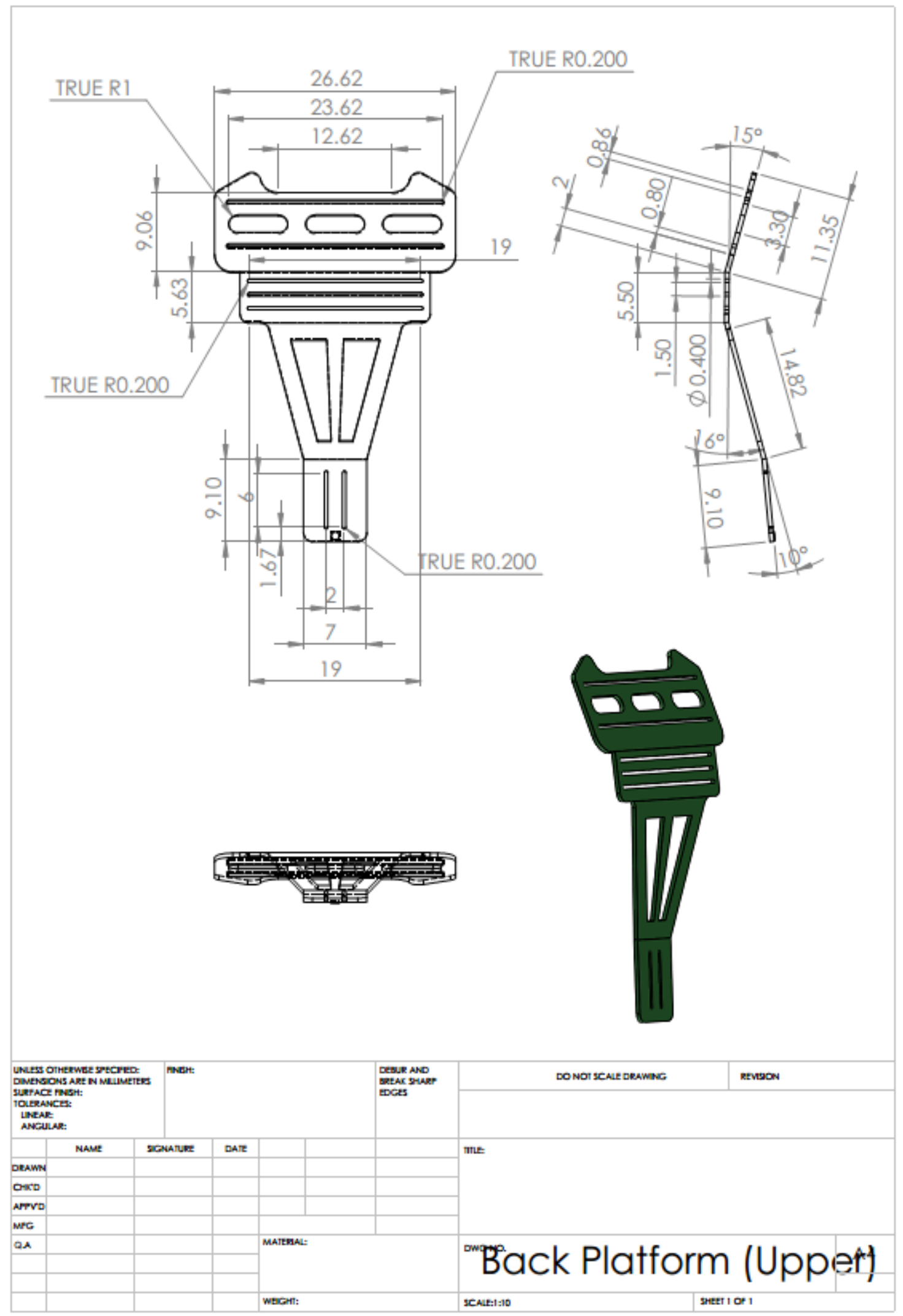




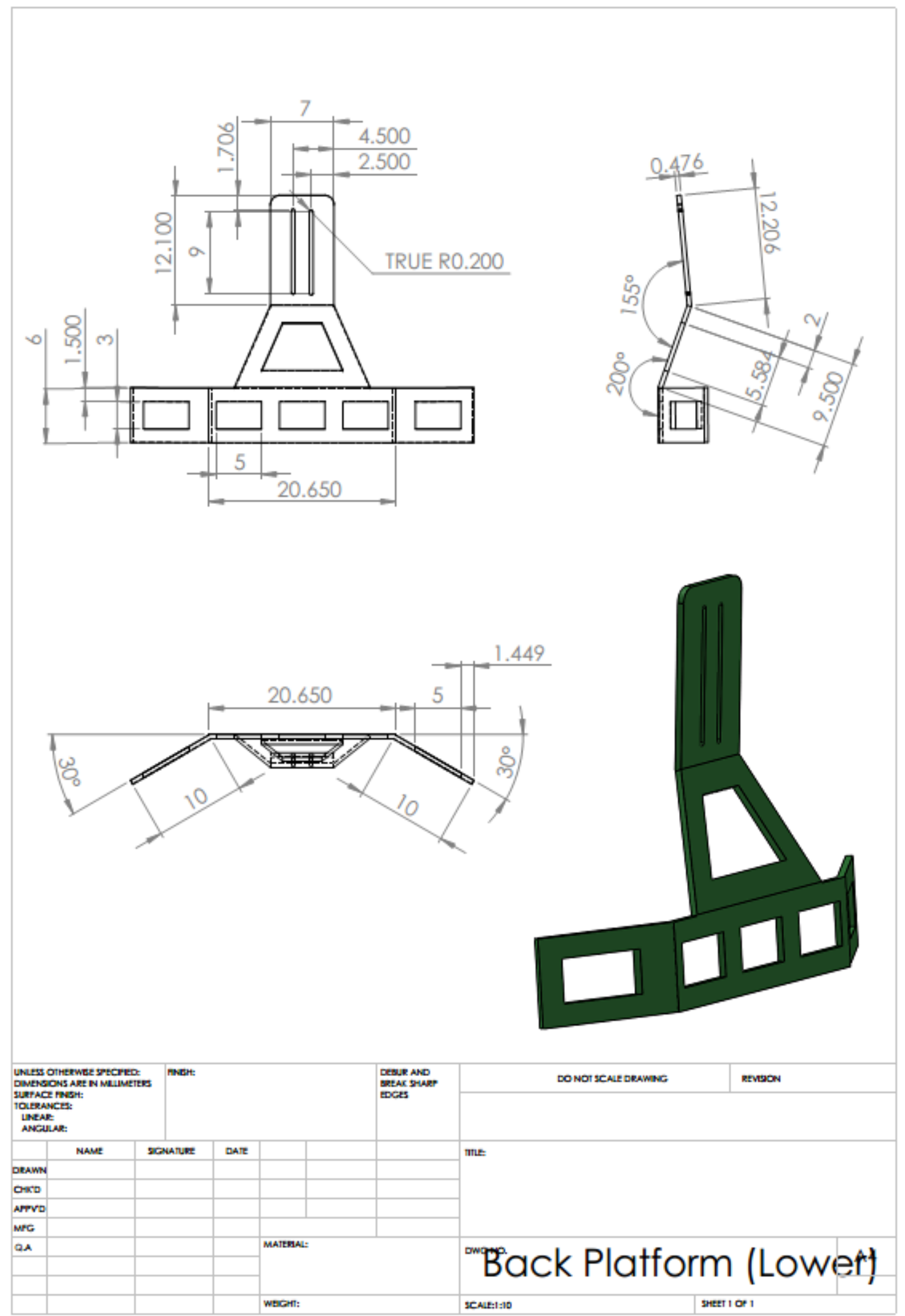




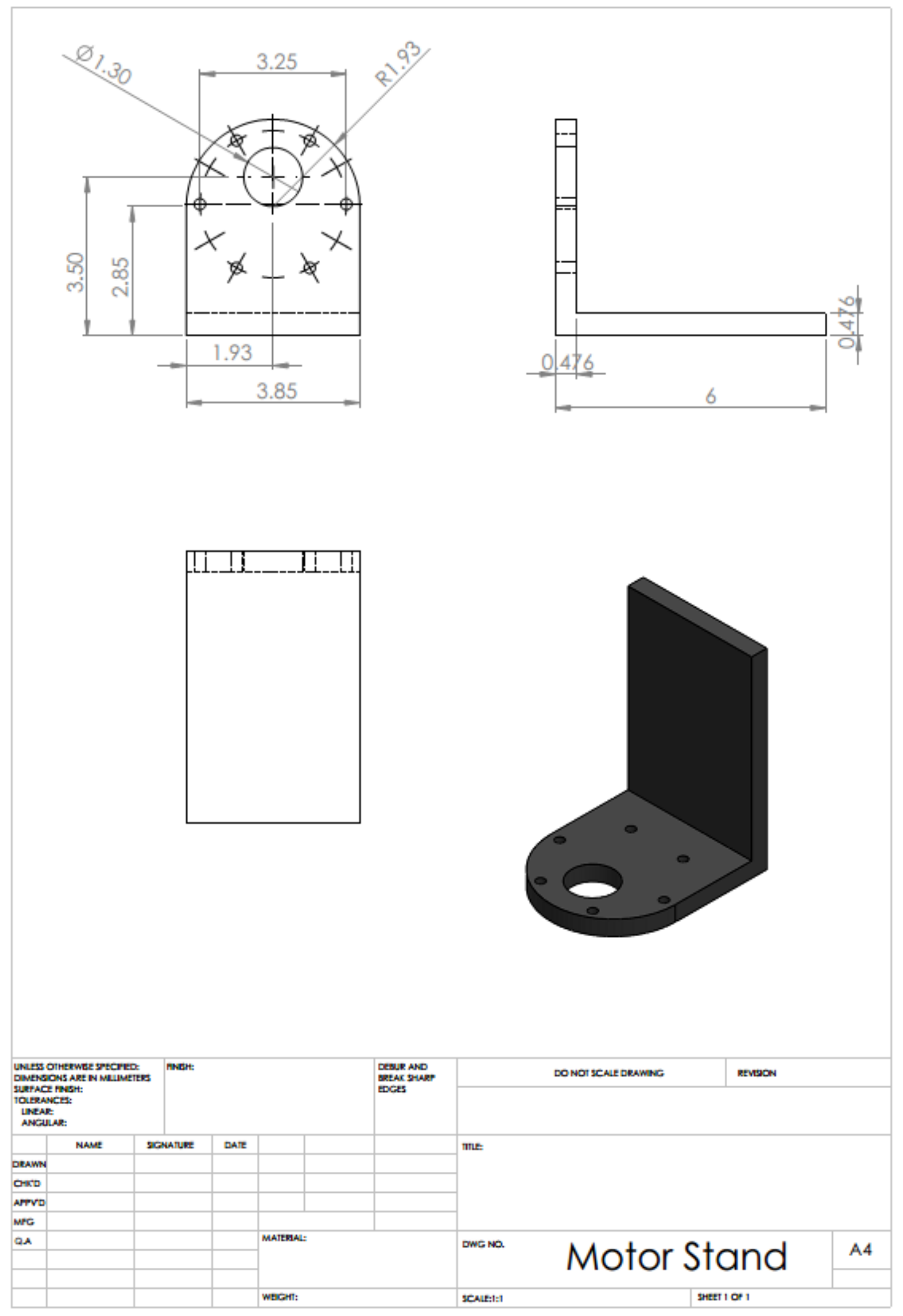




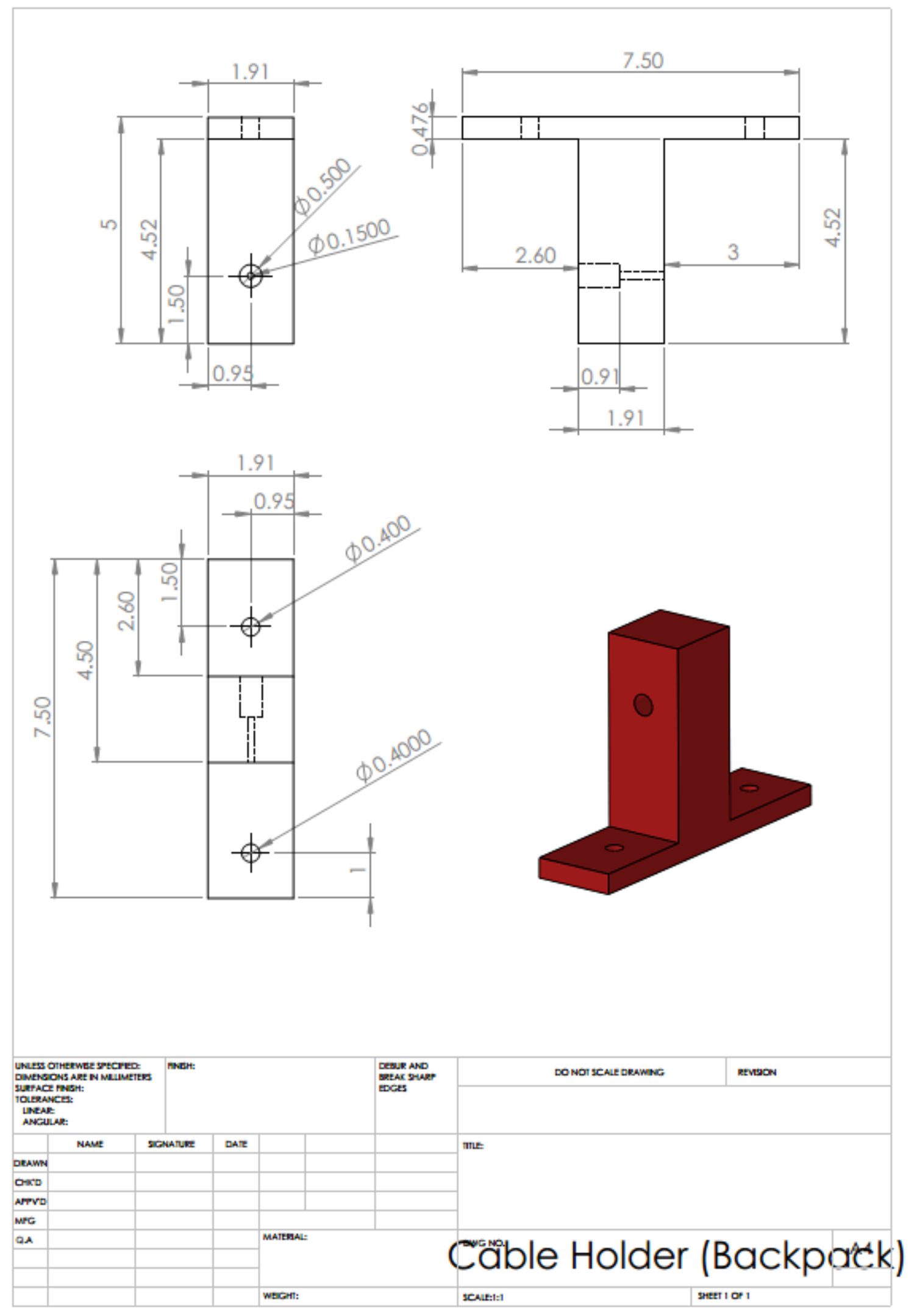




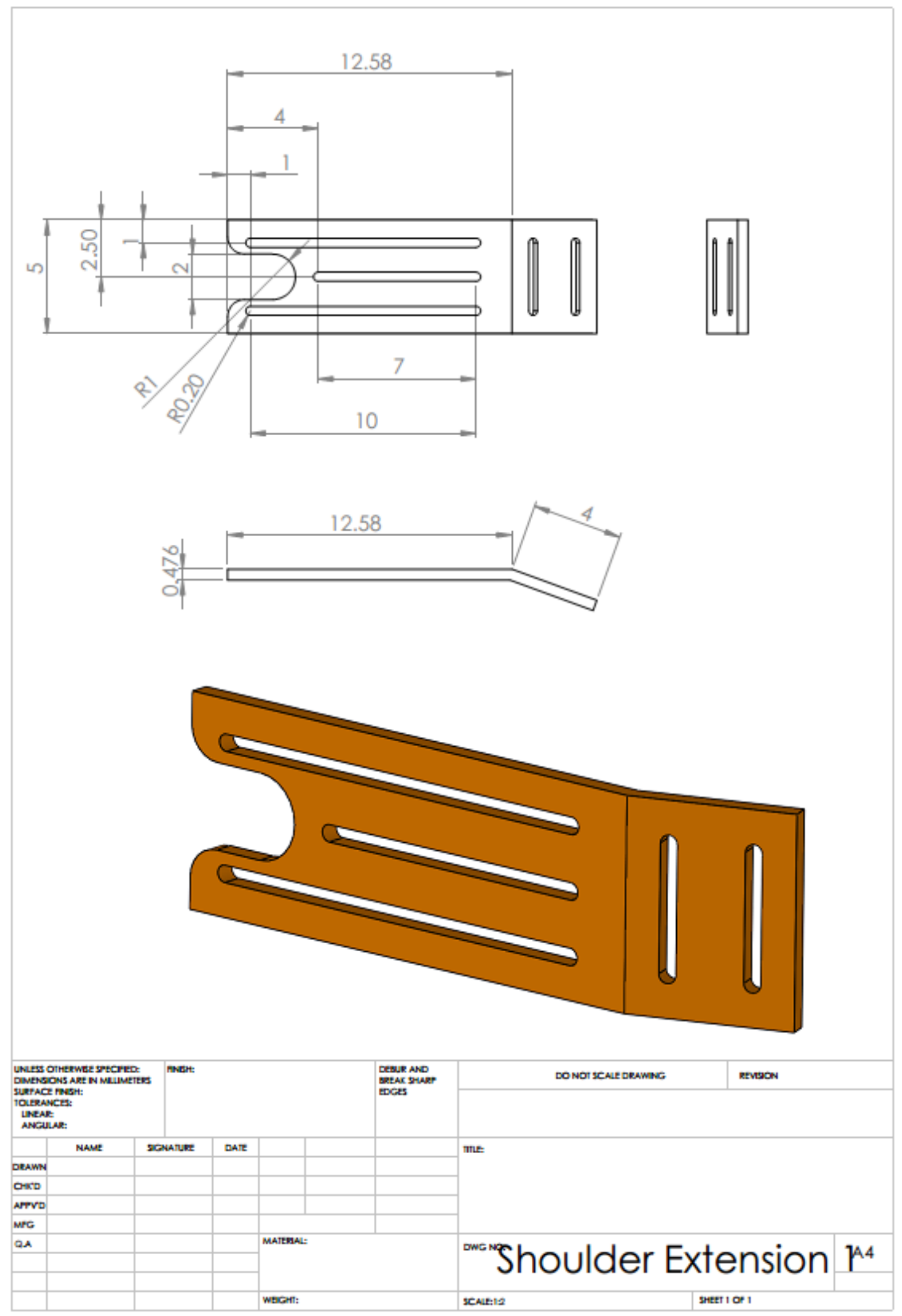




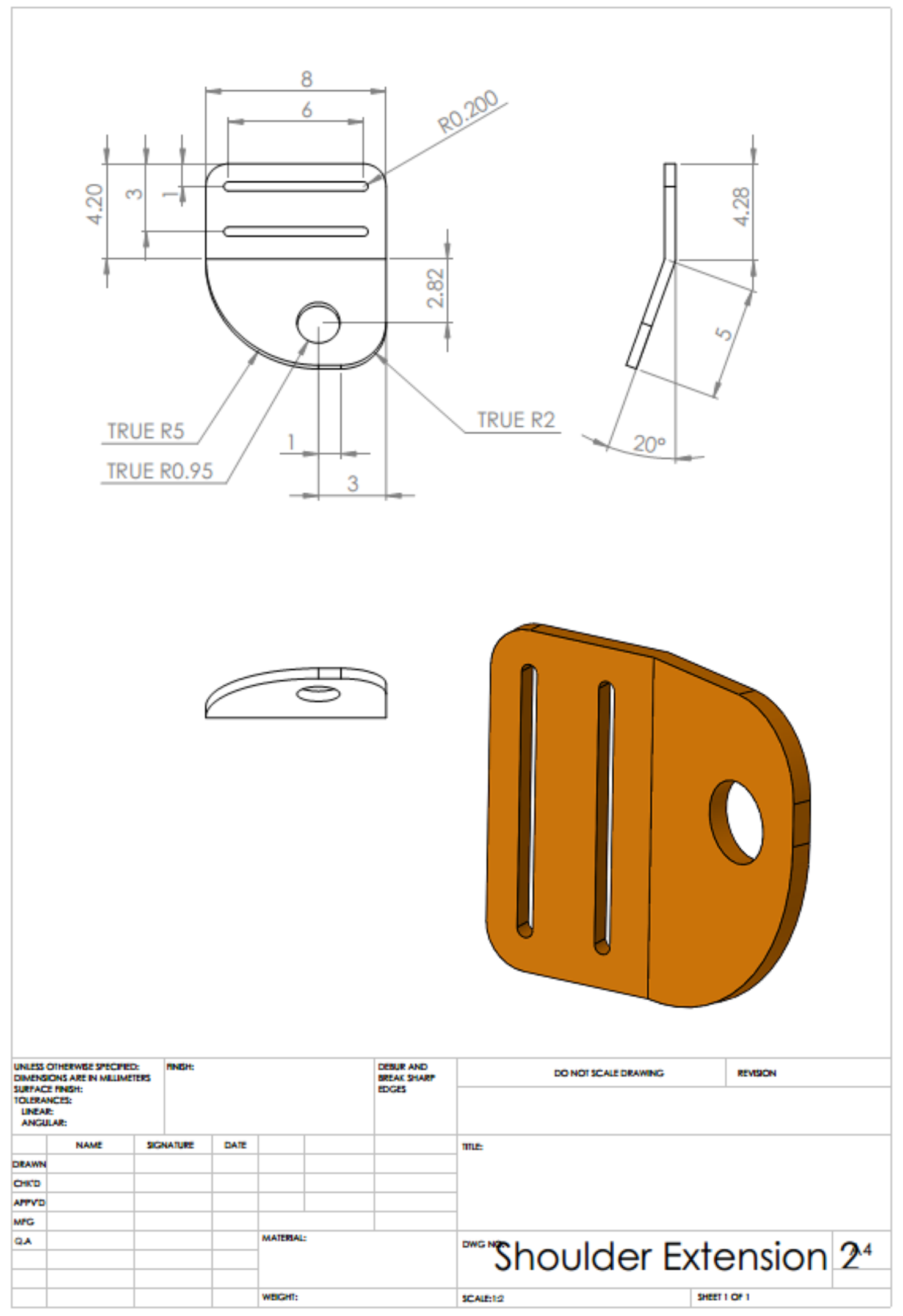




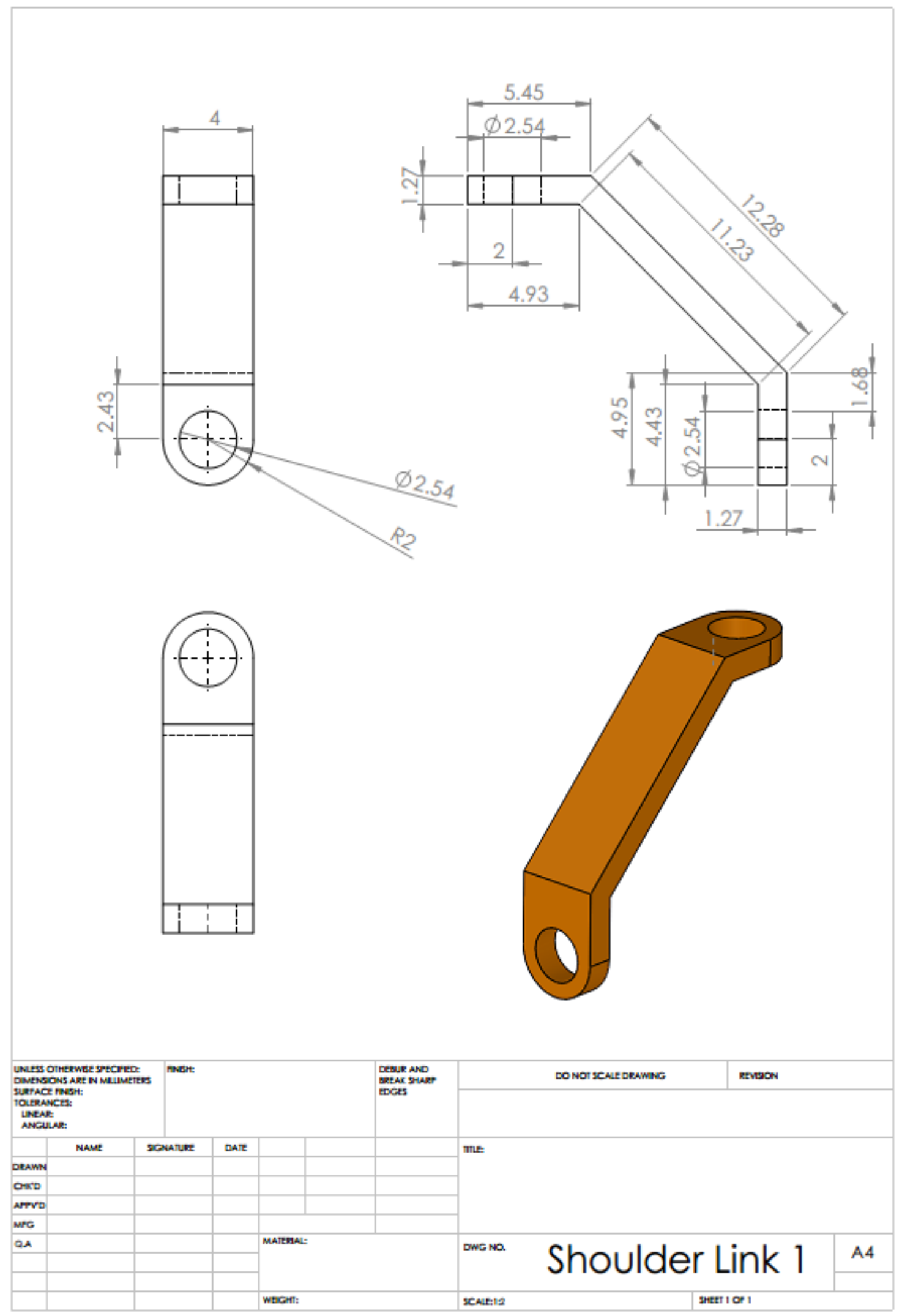




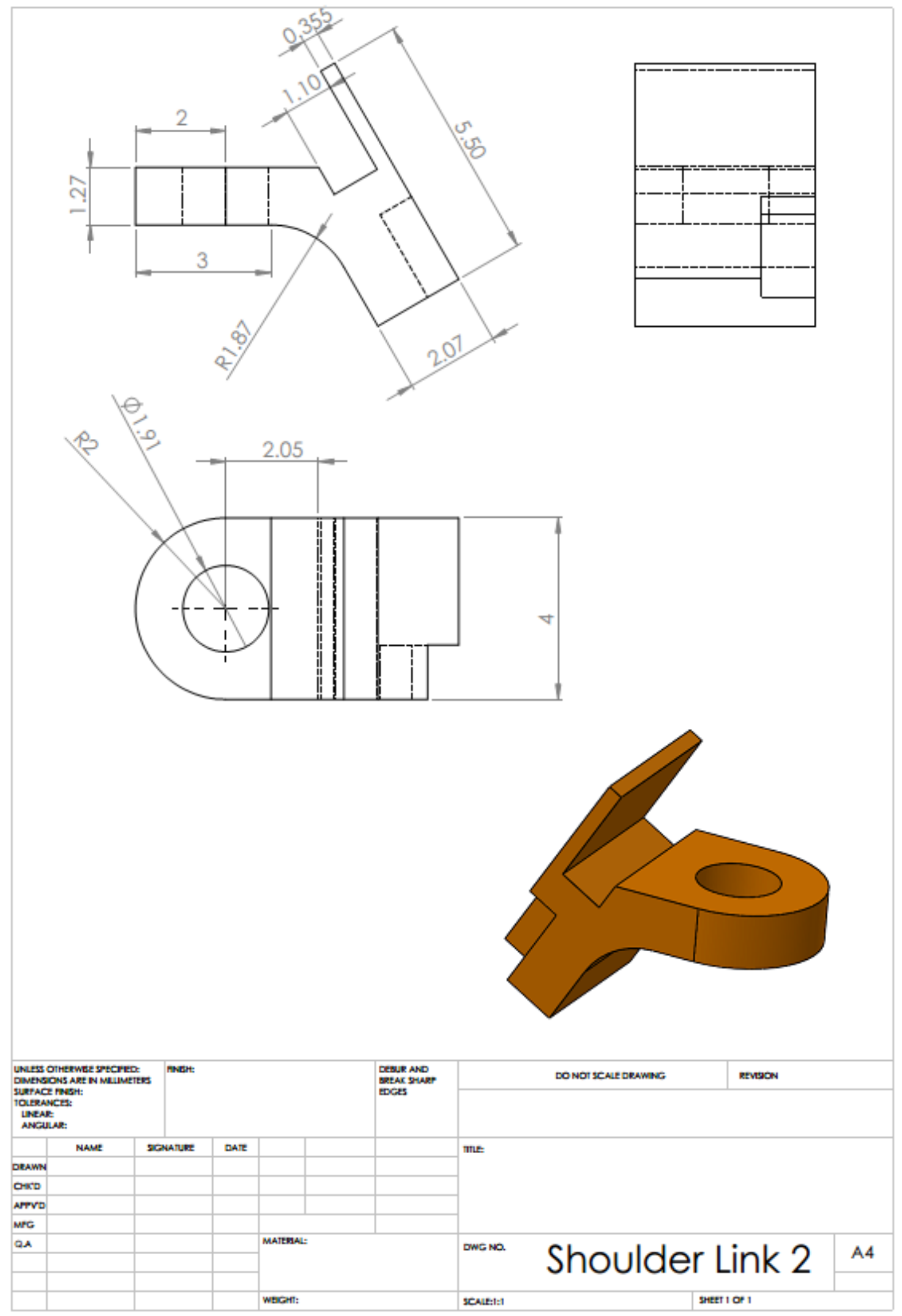




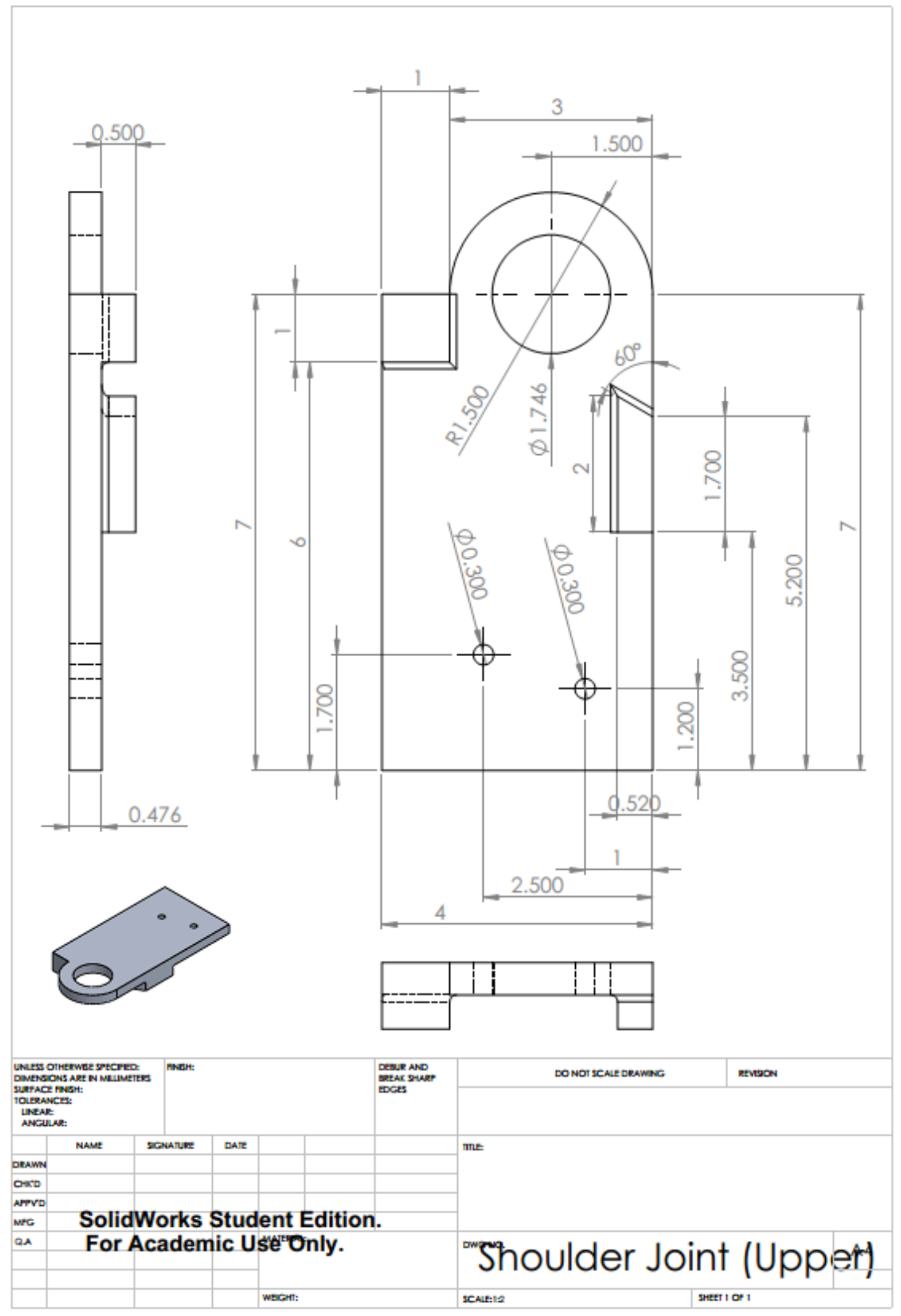




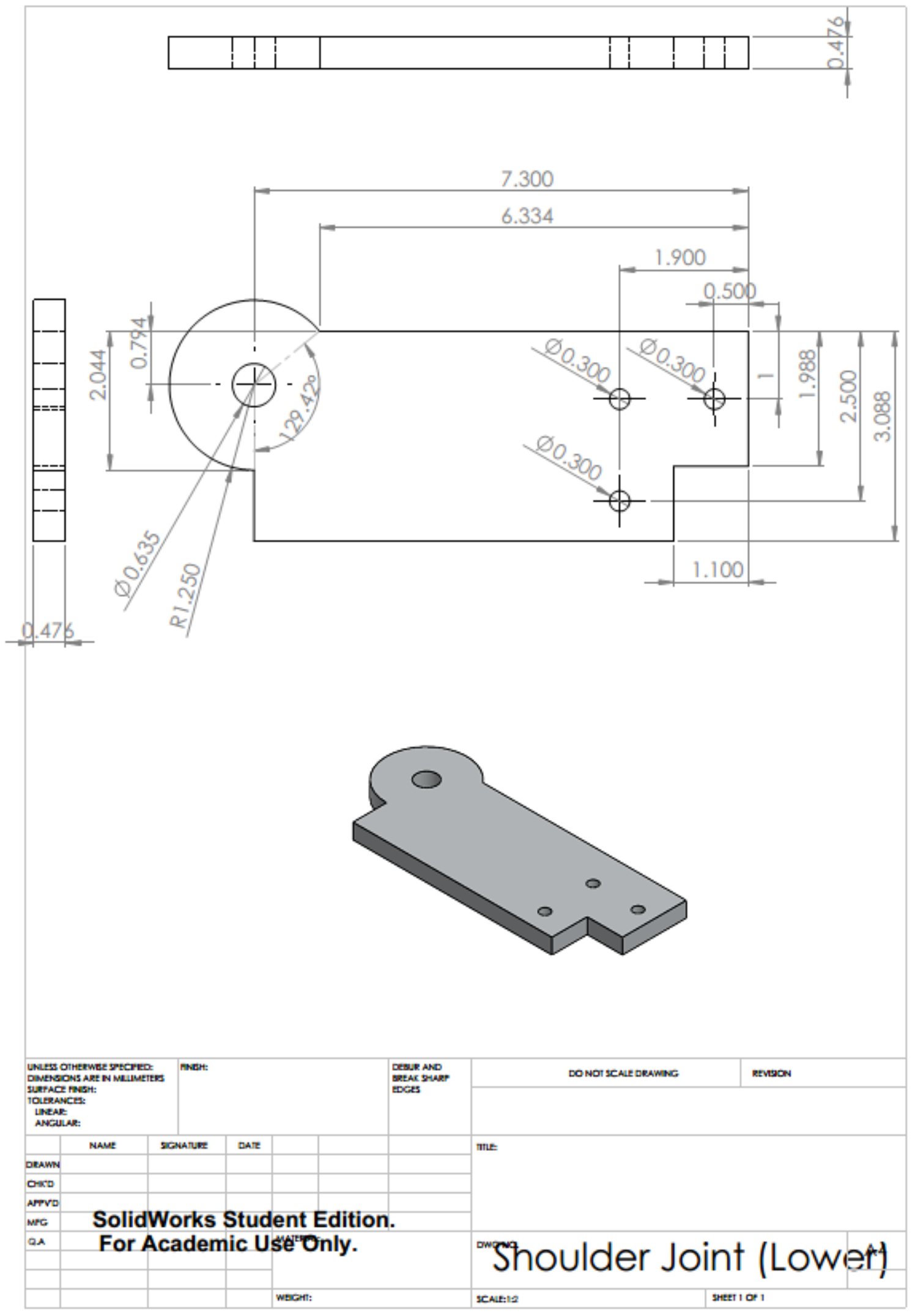




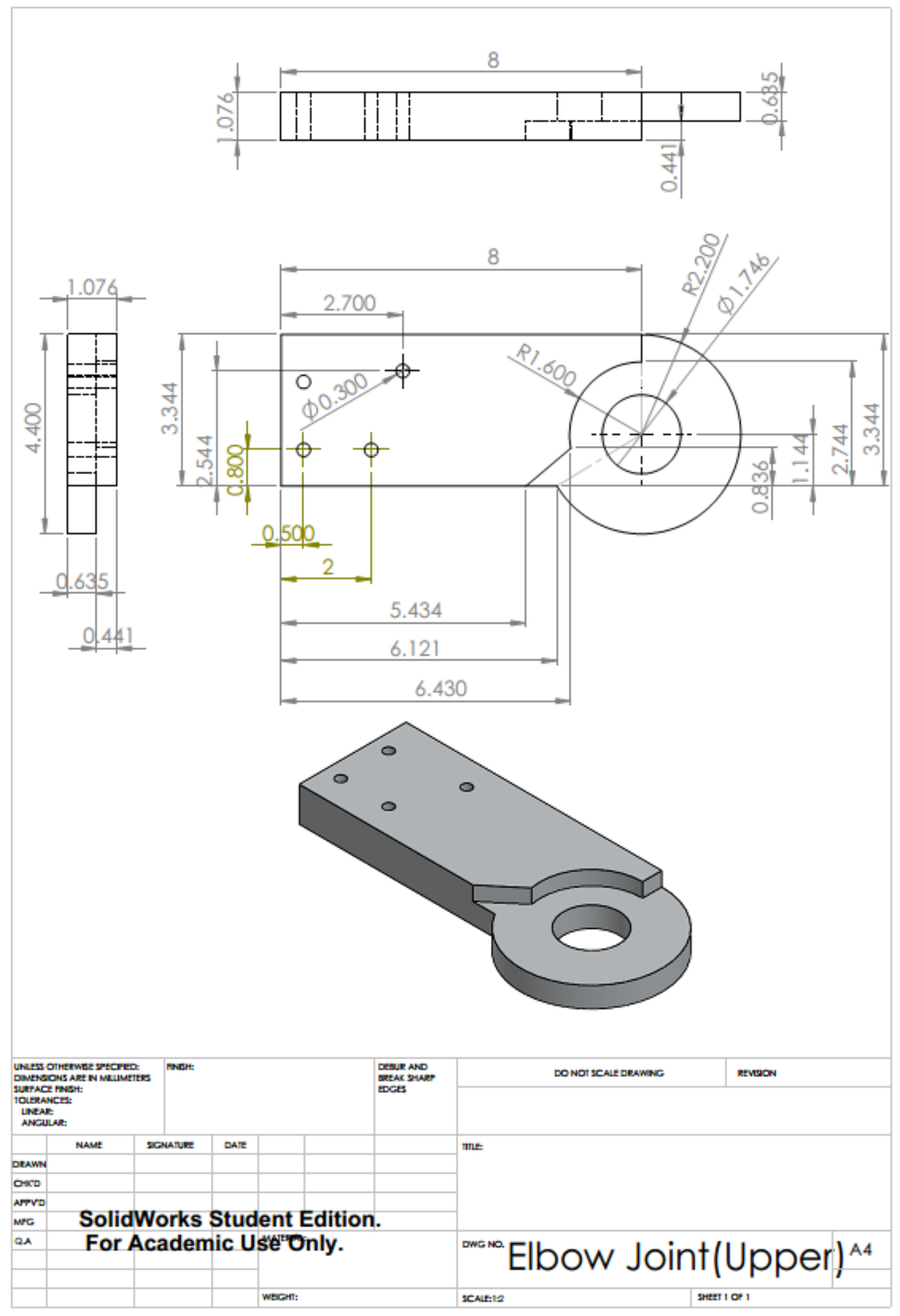




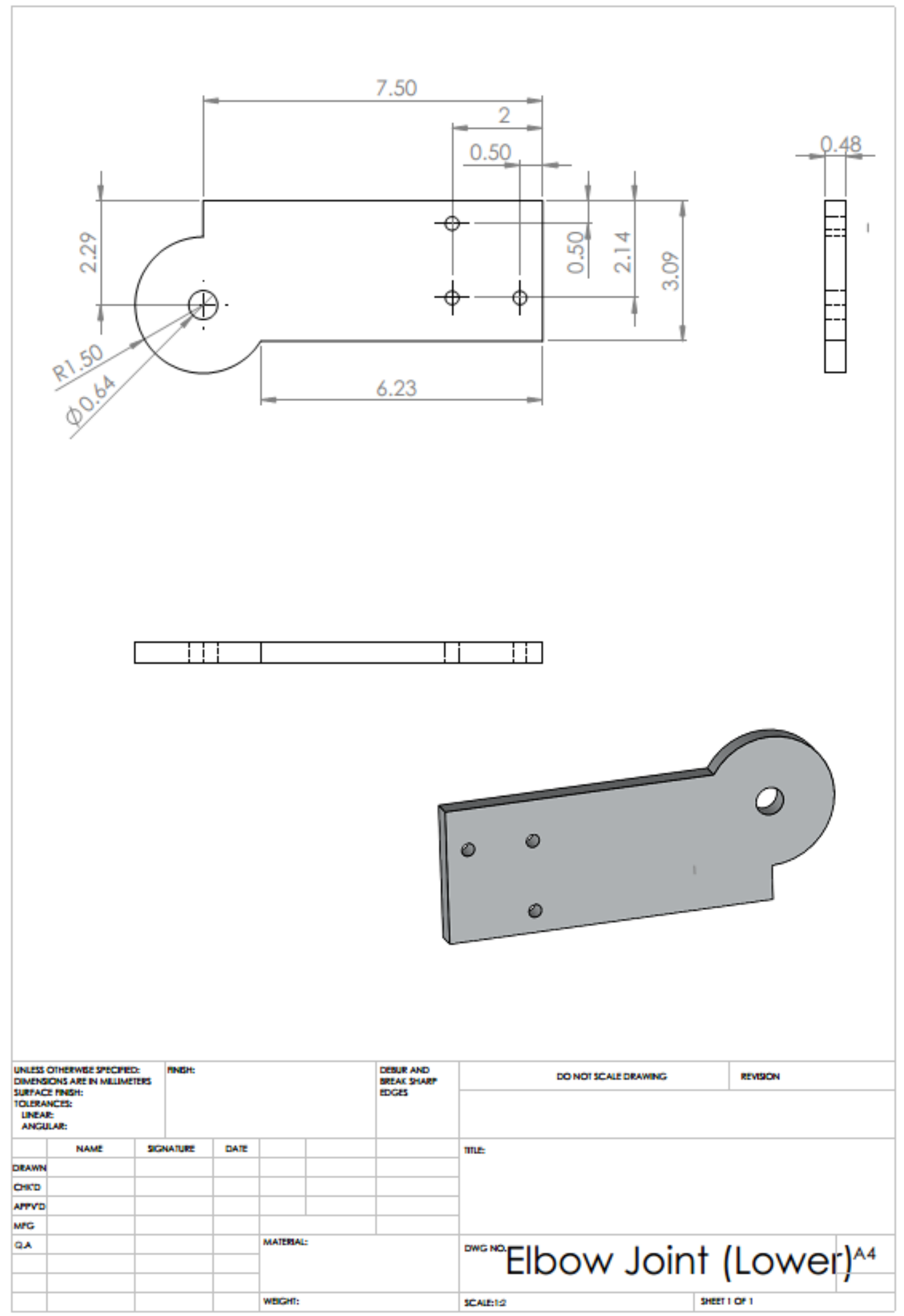




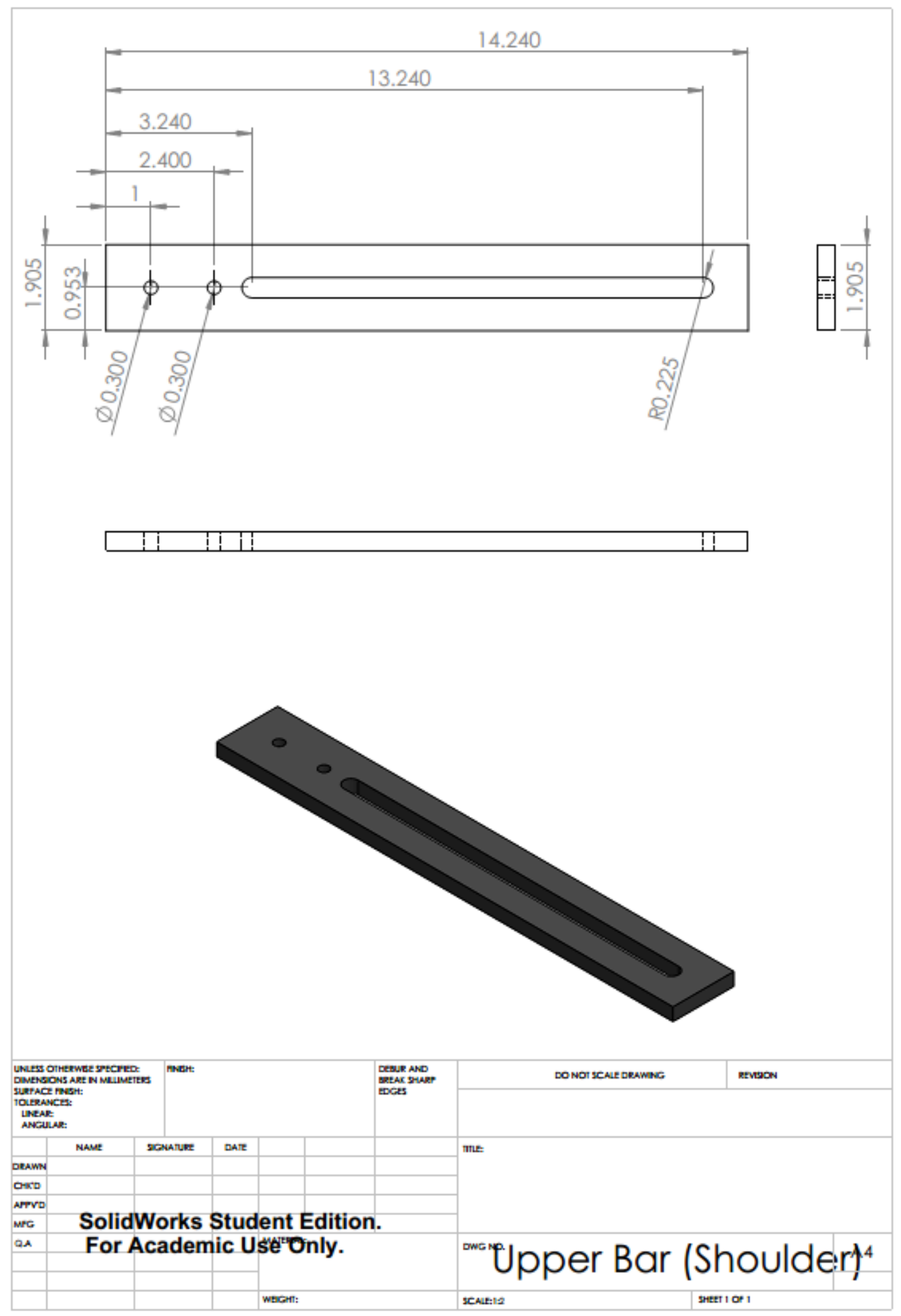




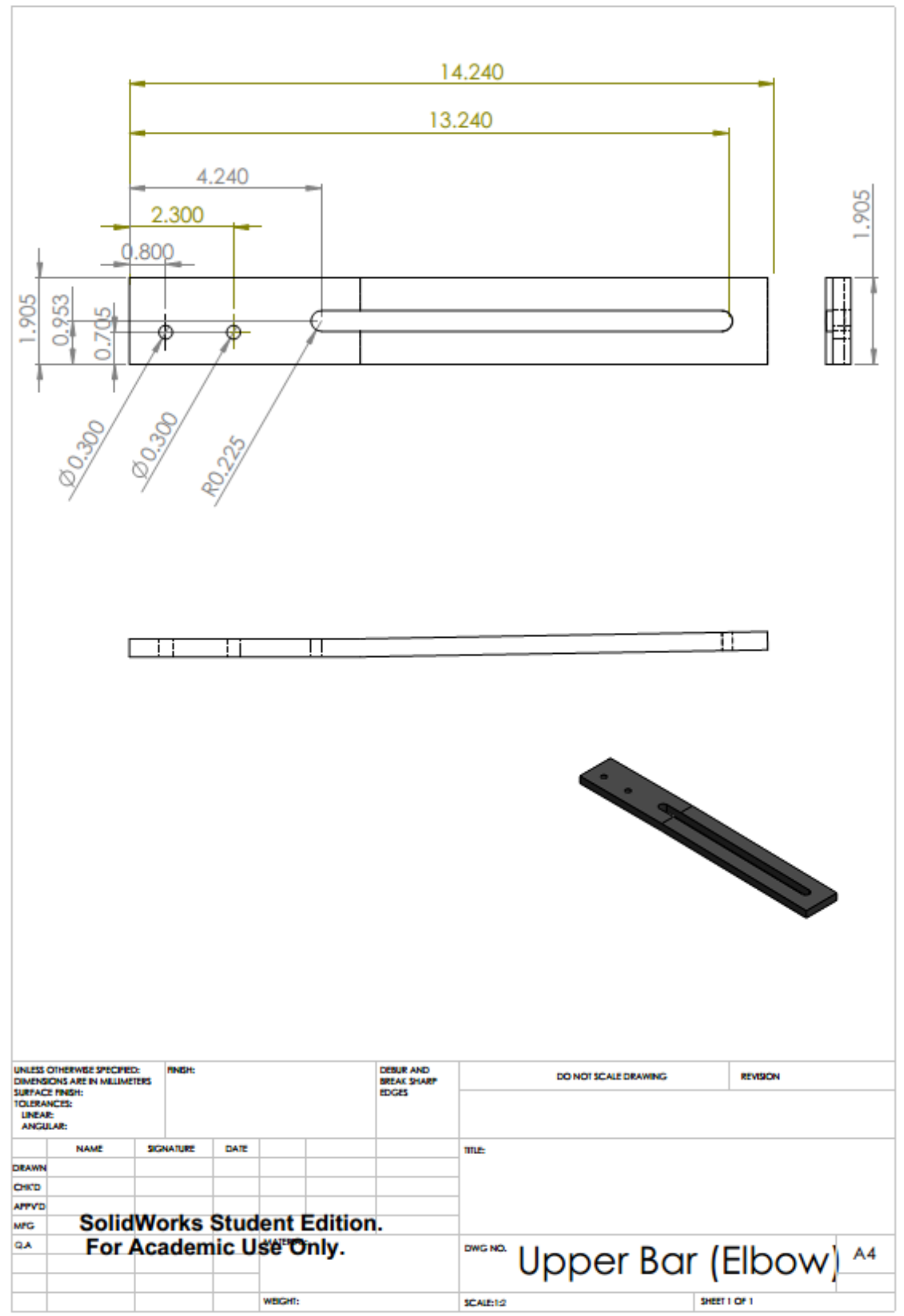




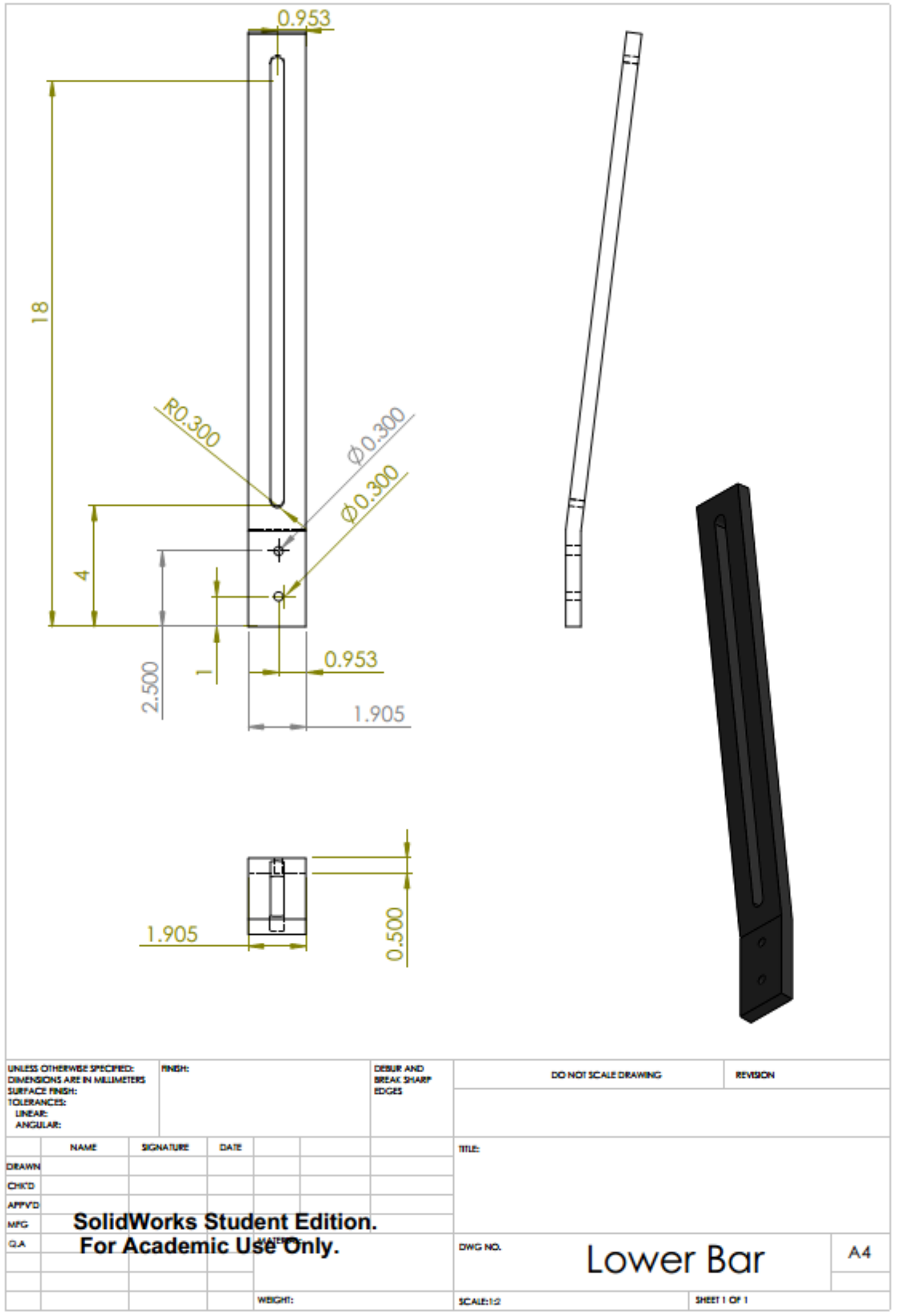




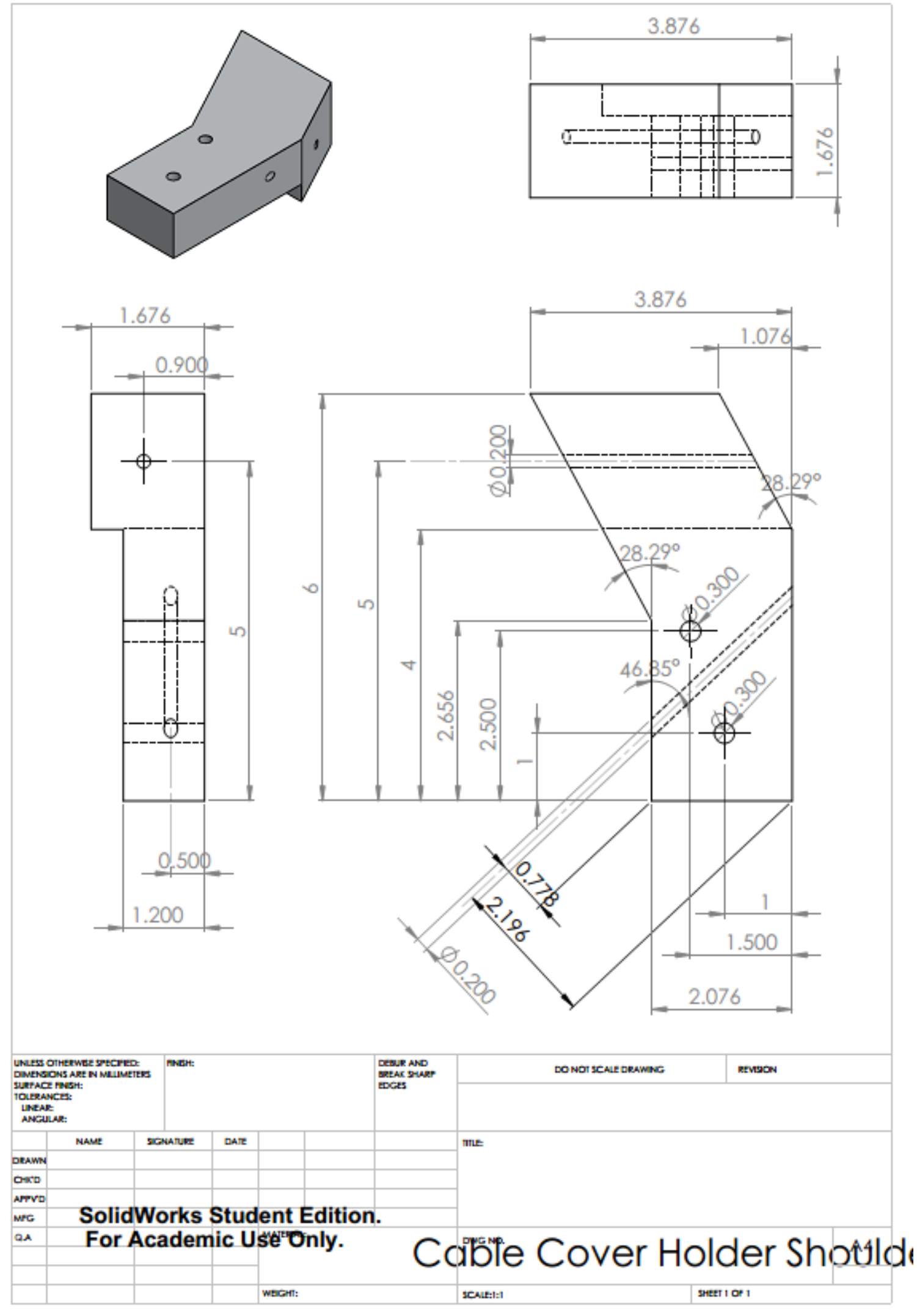




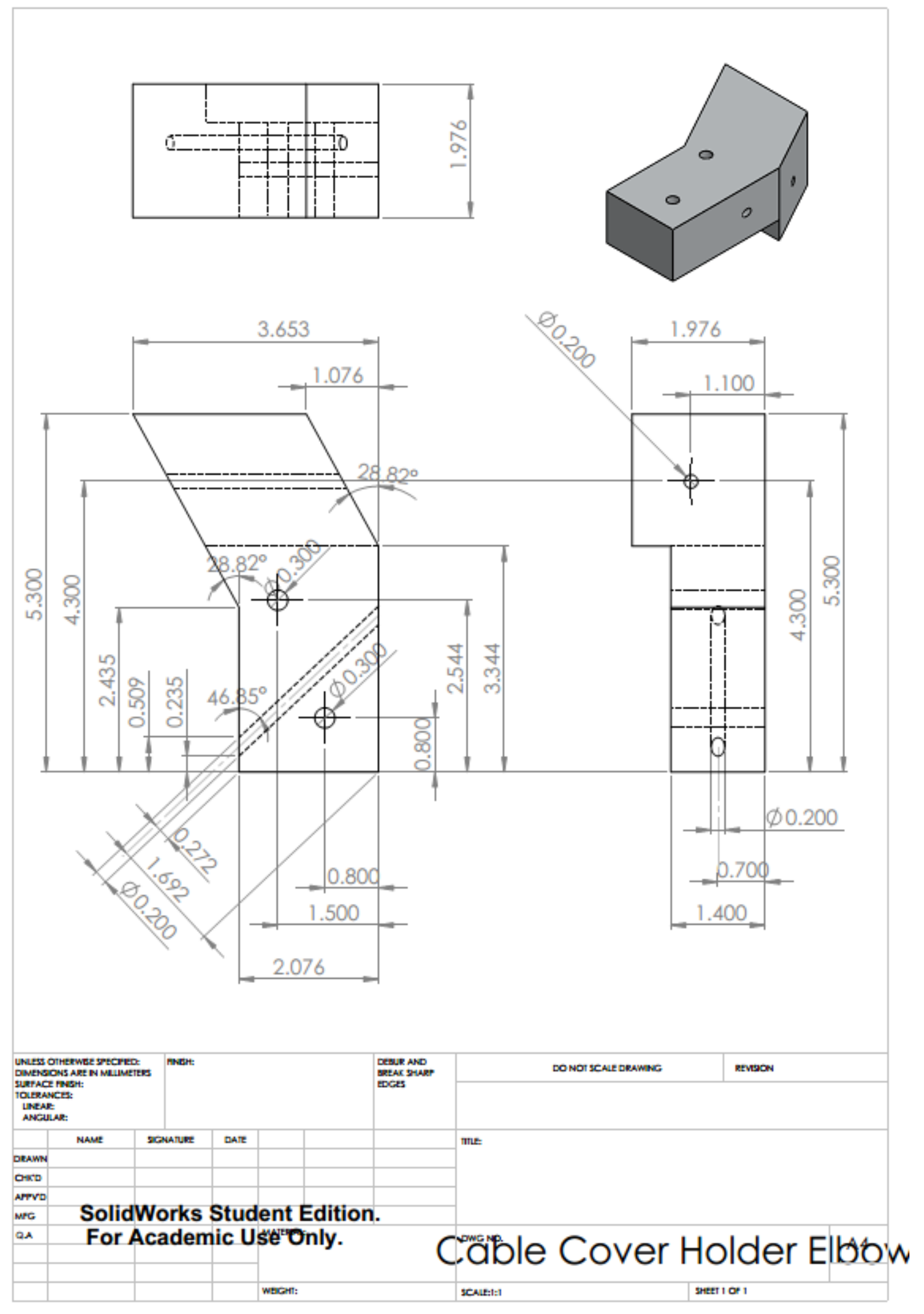




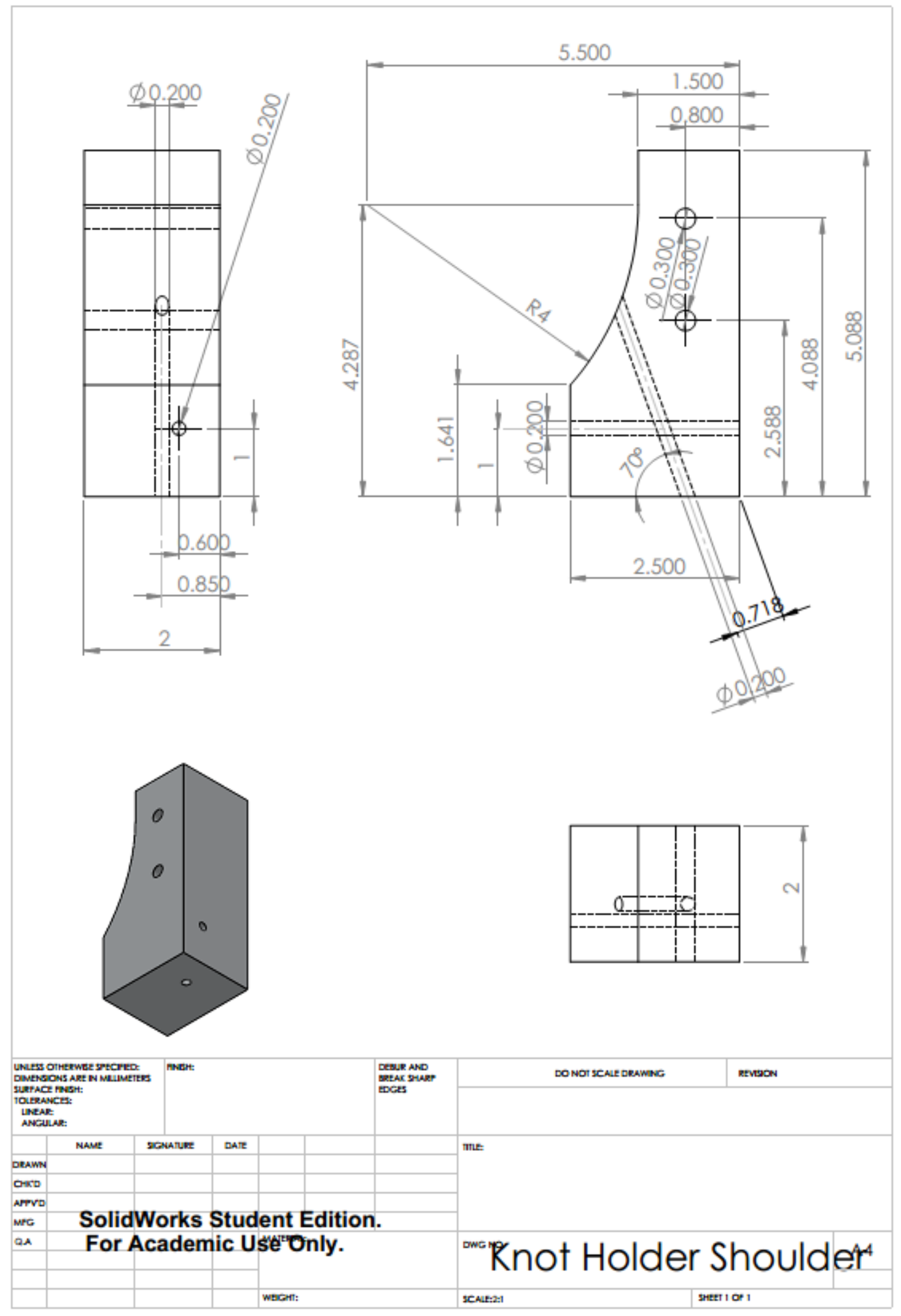




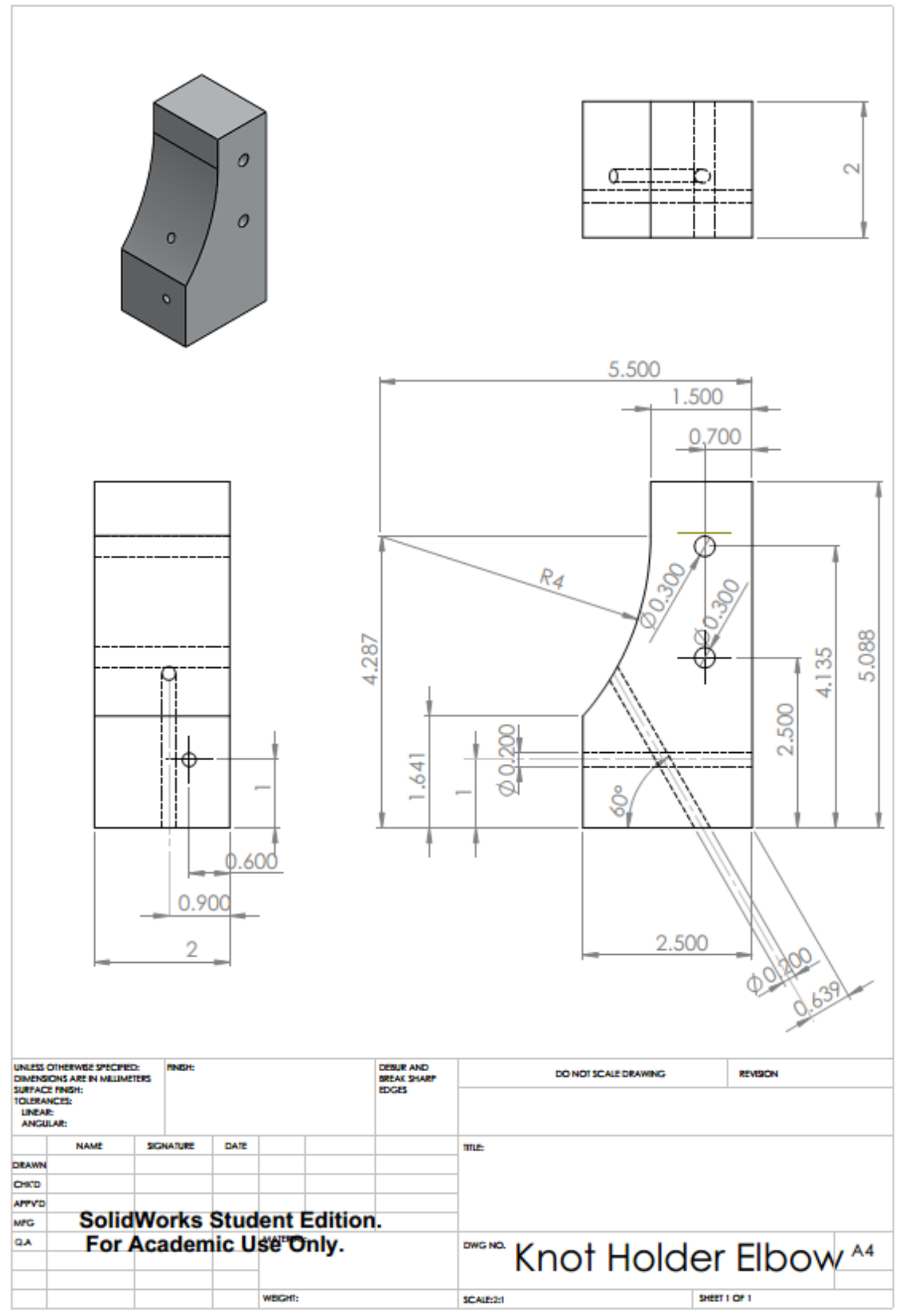




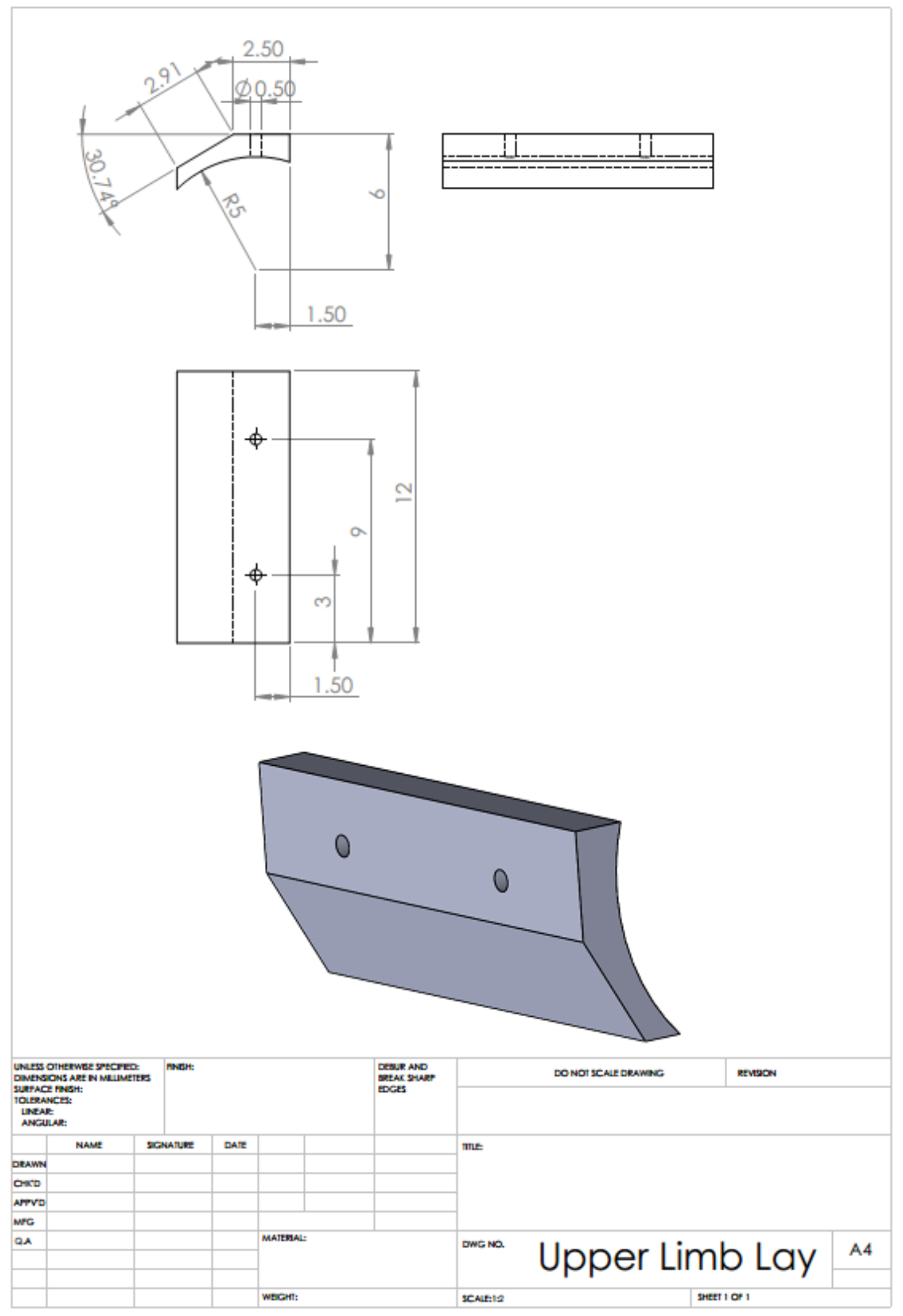




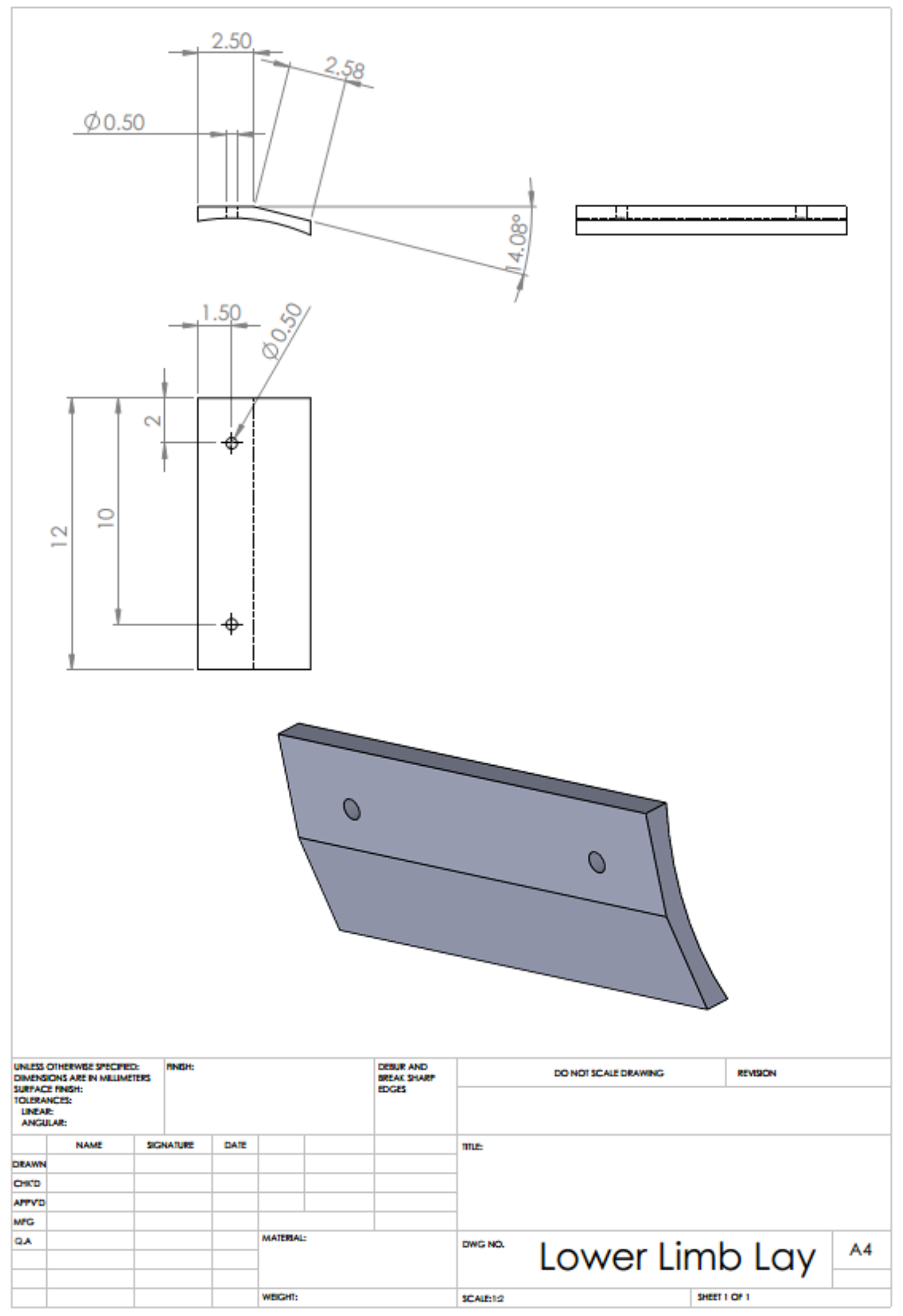


Appendix D Technical Specs of

\section{Instruments}




\section{D.1. 19:1 Metal Gear DC motor 37Dx52L mm with 64 CPR Encoder [223]}

This $2.54 " \times 1.45^{\prime \prime} \times 1.45^{\prime \prime}$ gearmotor is a powerful $12 \mathrm{~V}$ brushed DC motor with a 18.75:1 metal gearbox and an integrated quadrature encoder that provides a resolution of 64 counts per revolution of the motor shaft, which corresponds to 1200 counts per revolution of the gearbox's output shaft. These units have a 0.61 "-long, 6 mm-diameter D-shaped output shaft

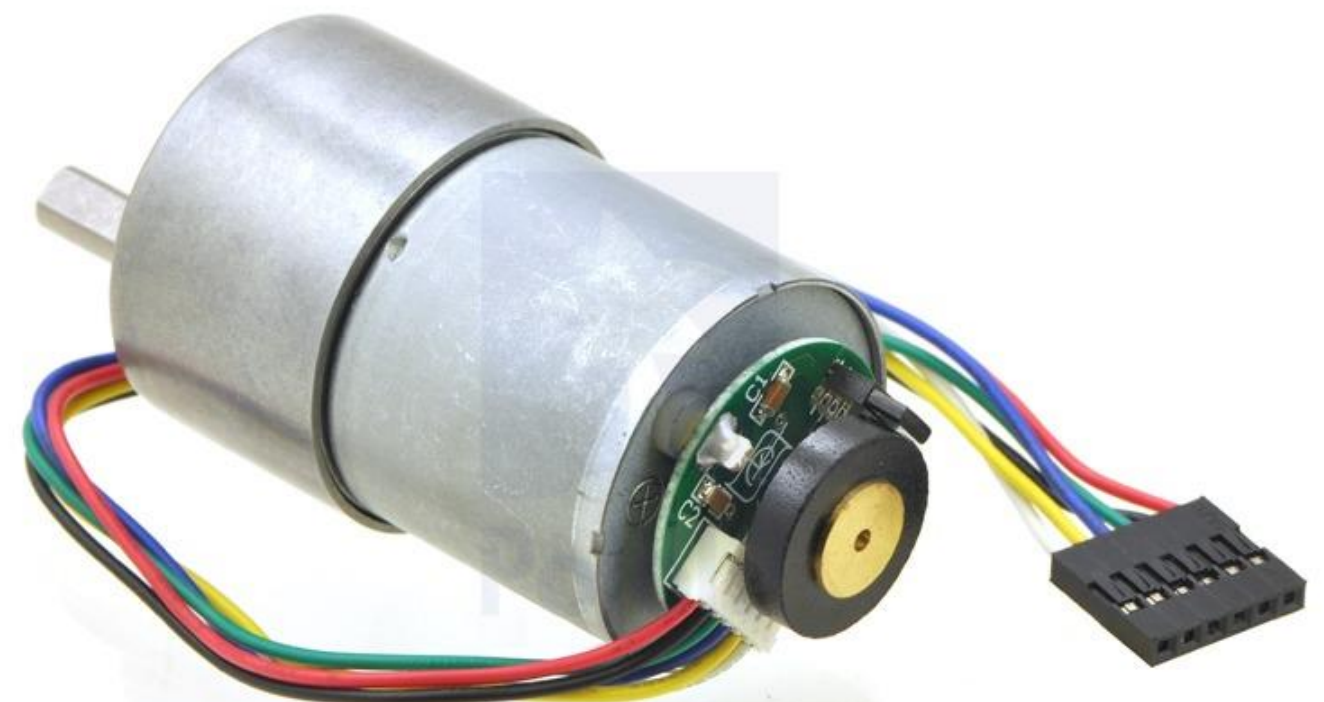

www.pololu.com

Figure D. 1. 19:1 Metal Gear DC motor 37Dx52L mm with 64 CPR Encoder

Key specs are: at 12 V: 500 RPM and $300 \mathrm{~mA}$ free-run, $84 \mathrm{oz}-\mathrm{in}(5 \mathrm{~kg}-\mathrm{cm})$ and $5 \mathrm{~A}$ stall.

Table D. 1. DC Motor Dimensions

\begin{tabular}{c|c} 
Size: & 37D x 52L mm \\
\hline Weight: & $6.7 \mathrm{oz}$
\end{tabular}




\begin{tabular}{l|l} 
Shaft diameter: & $6 \mathrm{~mm}$
\end{tabular}

Table D. 2. General specifications

Gear ratio: $\quad 19:$

Free-run speed @ 6V: 256 rpm1

Free-run current @ 6V: $\quad 250$ mA1

Stall current @ 6V: $\quad 2500$ mA1

Stall torque @ 6V: $\quad 42$ oz·in1

Free-run speed @ 12V: 500 rpm

Free-run current @ 12V: 300 mA

Stall current@12V: $\quad 5000$ mA

Stall torque @ 12V: 84 oz·in 


\title{
D.2. Angular Encoder HEDS-5500\#H06
}

\author{
HEDM-55xx/560x \& HEDS-55xx/56xx \\ Quick Assembly Two and Three Channel Optical Encoders
}

AVaGo

\section{Data Sheet}

HEDM-55x $x / 560 x$ HEDS-550x/554x, HEDS-560x/564x

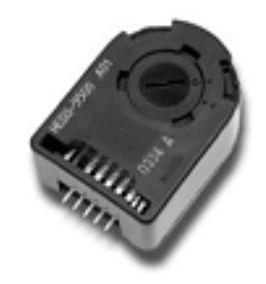

\section{Description}

The HEDS-5500/5540, HEDS-5600/5640, HEDM-5500/5540 and HEDM-5600 are high performance, low cost, two and three channel optical incremental encoders. These encoders emphasize high reliability, high resolution, and easy assembly.

Each encoder contains a lensed LED source, an integrated circuit with detectors and output circuitry, and a codewheel which rotates between the emitter and detector IC. The outputs of the HEDS-5500/5600 and HEDM-5500/ 5600 are two square waves in quadrature. The HEDS-5540/5640 and HEDM-5540 also have a third channel index output in addition to the two channel quadrature. This index output is a 90 electrical degree, high true index pulse which is generated once for each full rotation of the codewheel.

The HEDS series utilizes metal codewheels, while the HEDM series utilizes a film codewheel allowing for resolutions to 1024 CPR.

These encoders may be quickly and easily mounted to a motor. For larger diameter motors, the HEDM-5600, and HEDS-5600/5640 feature external mounting ears.

The quadrature signals and the index pulse are accessed through five 0.025 inch square pins located on 0.1 inch centers.

Standard resolutions between 96 and 1024 counts per revolution are presently available. Consult local Avago sales representatives for other resolutions.

\section{Features}

- Two channel quadrature output with optional index pulse

- Quick and easy assembly

- No signal adjustment required

- External mounting ears available

- Low cost

- Resolutions up to 1024 counts per revolution

- Small size $-40^{\circ} \mathrm{C}$ to $100^{\circ} \mathrm{C}$ operating temperature

- TTL compatible

- Single SV supply

\section{Applications}

The HEDS-5500, 5540, 5600, 5640, and the HEDM-5500, 5540,5600 provide motion detection at a low cost, making them ideal for high volume applications. Typical applications include printers, plotters, tape drives, positioning tables, and automatic handlers.

Note: Avago Technologies encoders are not recommended for use in safety critical applications. Eg. ABS braking systems, power steering, life support systems and critical care medical equipment. Please contact sales representative if more clarification is needed. 


\section{Package Dimensions}

\section{HEDS-5500/5540, HEDM-5500/5540}

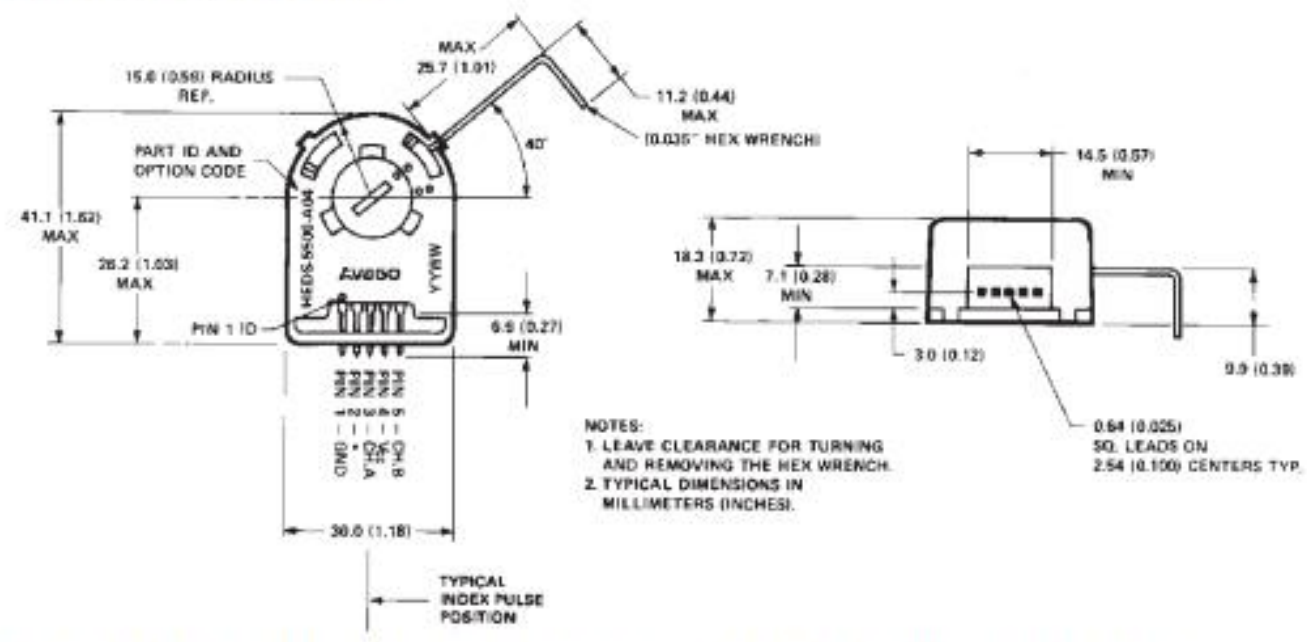

*Note: For the HEDS-5500 and HEDM-5S00, Pin $\approx 2$ is a No Connect. For the HEDS-5540 and HEOM-5540, Pin $\approx 2$ is CH. L, the index output.

HEDS-5600/5640, HEDM-5600

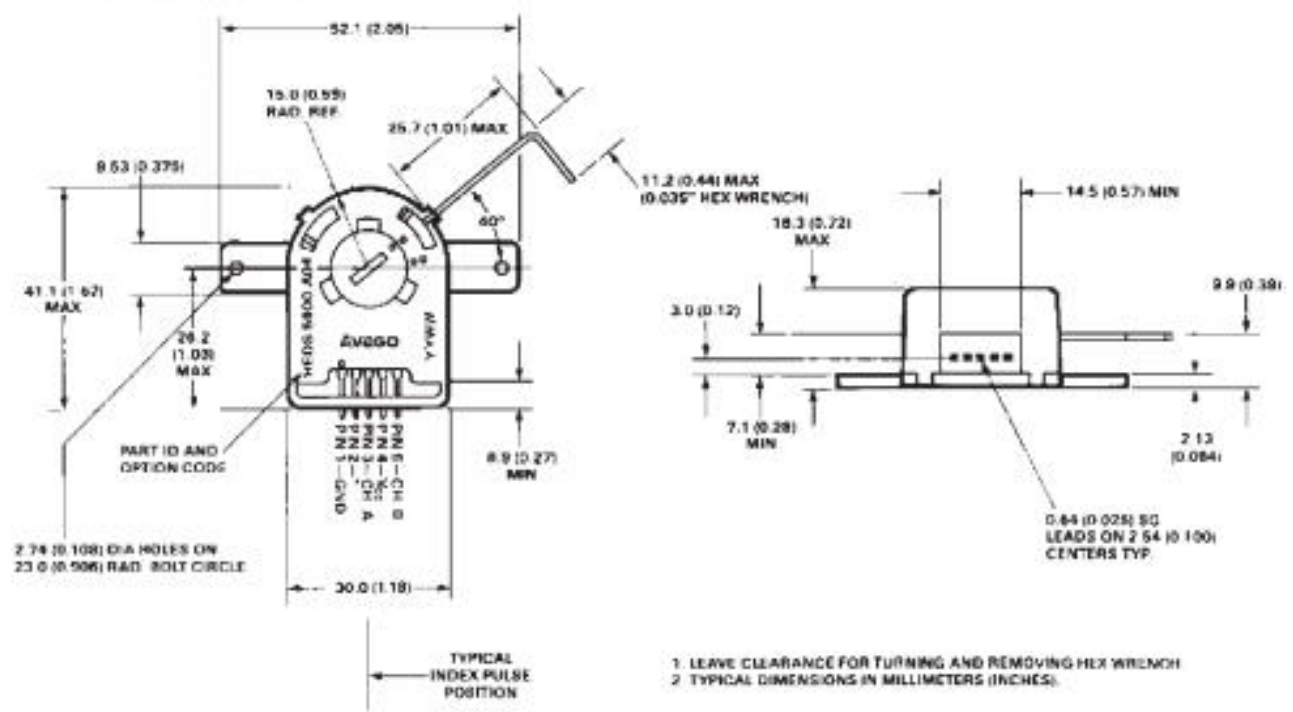

"Note: for the HEDS-5600 and HEDM-5600, Pin $\approx 2$ is a No Connect. For the MEDS-5640, Pin $\approx 2$ is $\mathrm{CH}$. L, the index output. 


\section{Package Dimensions}

HEDM / HEDS-5 $\times 8 \mathrm{x}$ and HEDM / HEDS-5 $\times 9 \mathrm{x}$
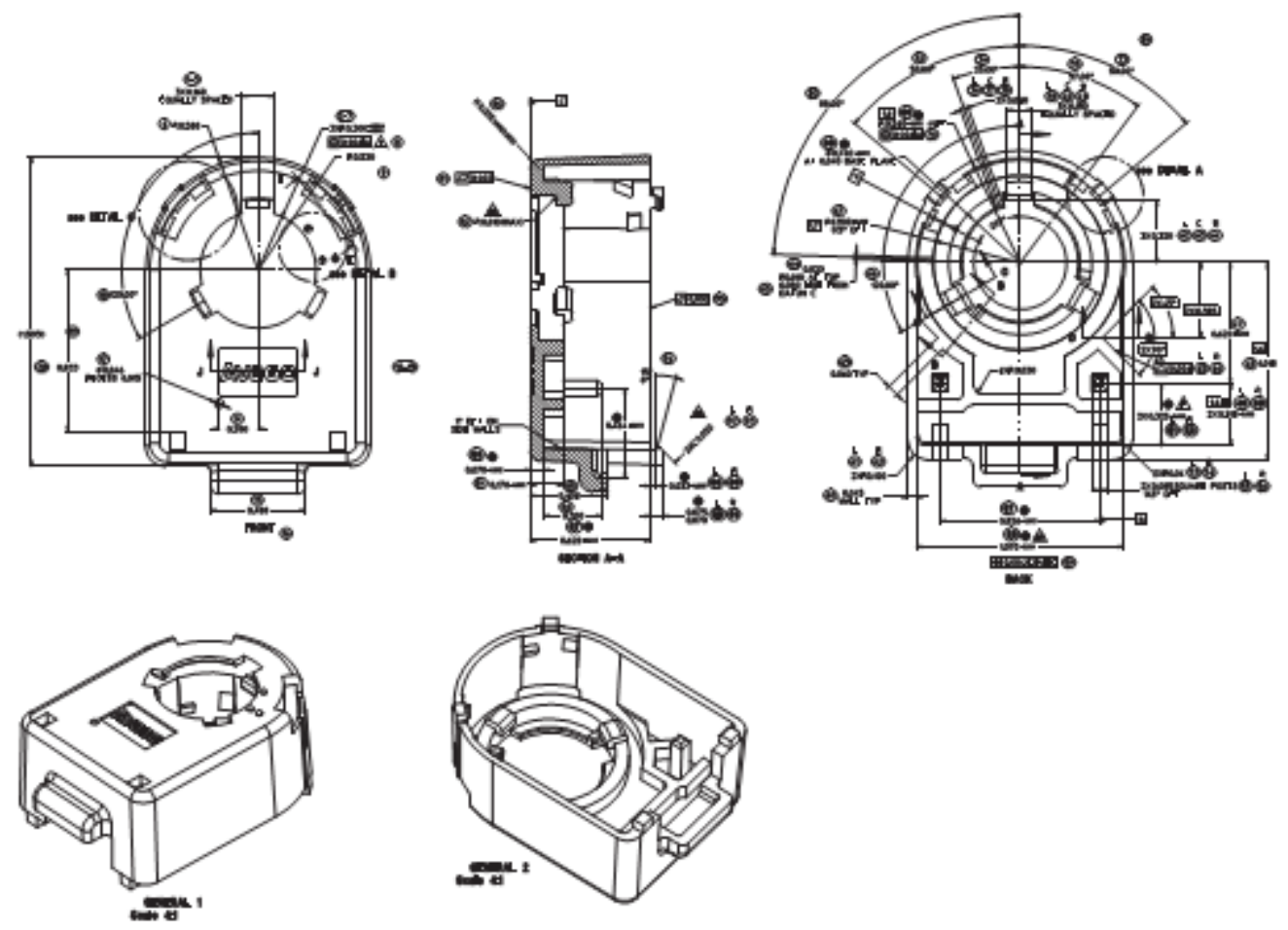


\section{Theory of Operation}

The HEDS-5500, 5540, 5600, 5640, and HEDM-5500, 5540, 5600 translate the rotary motion of a shaft into either a two- or a three-channel digital output.

As seen in the block diagram, these encoders contain a single Light Emitting Diode (LED) as its light source. The light is collimated into a parallel beam by means of a single polycarbonate lens located directly over the LED. Opposite the emitter is the integrated detector circuit. This IC consists of multiple sets of photodetectors and the signal processing circuitry necessary to produce the digital waveforms.

The codewheel rotates between the emitter and detector, causing the light beam to be interrupted by the pattem of spaces and bars on the codewheel. The photodiodes which detect these interruptions are arranged in a pattern that corresponds to the radius and design of the codewheel. These detectors are also spaced such that a light period on one pair of detectors corresponds to a dark period on the adjacent pair of detectors. The photodiode outputs are then-fed through the signal processing circuitry resulting in $A, A, B$ and $B$ (also $I$ and $I$ in the HEDS$5540 / 5640$ and HEDM-5540). Comparators receive these signals and produce the final outputs for channels $A$ and B. Due to this integrated phasing technique, the digital output of channel $\mathrm{A}$ is in quadrature with that of channel $B$ (90 degrees out of phase).

In the HEDS-5540/5640 and HEDM-5540, the output of the comparator for $I$ and $I$ is sent to the index processing circuitry along with the outputs of channels $A$ and $B$.

The final output of channel I is an index pulse PO which is generated once for each full rotation of the codewheel. This output PO is a one state width (nominally 90 electrical degrees), high true index pulse which is coincident with the low states of channels $A$ and $B$.

\section{Block Diagram}

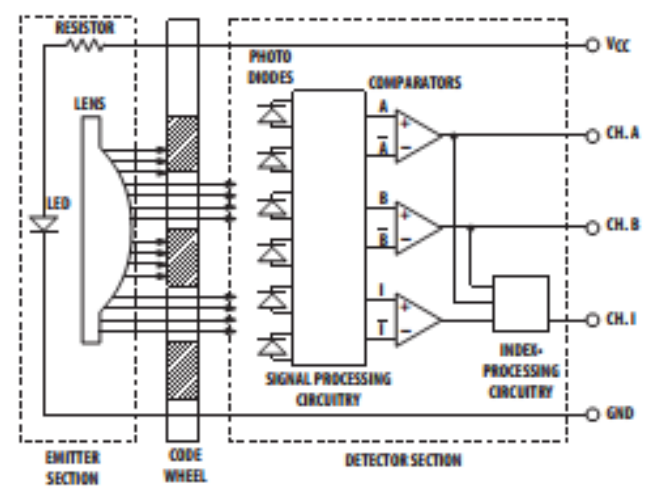

\section{Definitions}

Count $(\mathrm{N})$ : The number of bar and window pairs or counts per revolution (CPR) of the codewheel.

One (yde (C): 360 electrical degrees ("e), 1 bar and window pair.

One Shaft Rotation: 360 mechanical degrees, $\mathrm{N}$ cycles.

Position Error $(\Delta \theta)$ : The normalized angular difference between the actual shaft position and the position indicated by the encoder cycle count.

Cyde Error ( $\triangle C$ ): An indication of cycle uniformity. The differ $\neg$ ence between an observed shaft angle which gives rise to one electrical cycle, and the nominal angular increment of $1 / \mathrm{N}$ of a revolution.

Pulse Width (P): The number of electrical degrees that an output is high during $1 \mathrm{cycle}$. This value is nominally $180^{\circ} \mathrm{e}$ or $1 / 2$ cycle.

Pulse Width Error ( $\triangle \mathrm{P}$ ): The deviation, in electrical degrees, of the pulse width from its ideal value of $180^{\circ} \mathrm{e}$

State Width (\$): The number of electrical degrees between a transition in the output of channel $A$ and the neighbouring transition in the output of channel B. There are 4 states per cycle, each nominally $90^{\circ} \mathrm{e}$.

State Width Error ( $\Delta S)$ : The deviation, in electrical degrees, of each state width from its ideal value of $90^{\circ} \mathrm{e}$.

Phase $(\varphi)$ : The number of electrical degrees between the center of the high state of channel A and the center of the high state of channel B. This value is nominally $90^{\circ} \mathrm{e}$ for quadrature output.

Phase Error $(\Delta \varphi)$ : The deviation of the phase from its ideal value of $90^{\circ} \mathrm{e}$.

Direction of Rotation: When the codewheel rotates in the counter-clockwise direction (as viewed from the encoder end of the motor), channel A will lead channel B. If the codewheel rotates in the clockwise direction, channel $\mathrm{B}$ will lead channel $A$.

Index Pulse Width $\left(P_{0}\right)$ : The number of electrical degrees that an index output is high during one full shaft rotation. This value is nominally $90^{\circ} \mathrm{e}$ or $1 / 4$ cycle. 


\section{Absolute Maximum Ratings}

\begin{tabular}{|c|c|c|c|}
\hline Parameter & HEDS-55XX/56) $\alpha$ & HEDM-550X/560X & HEDM-5540 \\
\hline Storage Temperature, Ts & $-40^{\circ} \mathrm{C}$ to $100^{\circ} \mathrm{C}$ & $-40^{\circ} \mathrm{C}$ to $+70^{\circ} \mathrm{C}$ & $-40^{\circ} \mathrm{C}$ to $85^{\circ} \mathrm{C}$ \\
\hline Operating Temperature, $T_{A}$ & $-40^{\circ} \mathrm{C}$ to $100^{\circ} \mathrm{C}$ & $-40^{\circ} \mathrm{C}$ to $+70^{\circ} \mathrm{C}$ & $-40^{\circ} \mathrm{C}$ to $85^{\circ} \mathrm{C}$ \\
\hline Supply Voltage, Vcc & $-0.5 \mathrm{~V}$ to $7 \mathrm{~V}$ & $-0.5 \mathrm{~V}$ to $7 \mathrm{~V}$ & $-0.5 \mathrm{~V}$ to $7 \mathrm{~V}$ \\
\hline Output Voltage, $V_{O}$ & $-0.5 \mathrm{~V}$ to VCC & $-0.5 \mathrm{~V}$ to VCC & $-0.5 \mathrm{~V}$ to VCC \\
\hline Output Current per Channel, Iour & $-1.0 \mathrm{~mA}$ to $5 \mathrm{~mA}$ & $-1.0 \mathrm{~mA}$ to $5 \mathrm{~mA}$ & $-1.0 \mathrm{~mA}$ to $5 \mathrm{~mA}$ \\
\hline Vibration & $20 \mathrm{~g}, 5$ to $1000 \mathrm{~Hz}$ & $20 \mathrm{~g}, 5$ to $1000 \mathrm{~Hz}$ & $20 \mathrm{~g}, 5$ to $1000 \mathrm{~Hz}$ \\
\hline Shaft Axial Play & $\begin{array}{l} \pm 0.25 \mathrm{~mm} \\
( \pm 0.010 \mathrm{in} .)\end{array}$ & $\begin{array}{l} \pm 0.175 \mathrm{~mm} \\
( \pm 0.007 \mathrm{in} .)\end{array}$ & $\begin{array}{l} \pm 0.175 \mathrm{~mm} \\
( \pm 0.007 \mathrm{in} .)\end{array}$ \\
\hline Shaft Eccentricity Plus Radial Play & $\begin{array}{l}0.1 \mathrm{~mm} \\
(0.004 \mathrm{in} .) \mathrm{TIR}\end{array}$ & $\begin{array}{l}0.04 \mathrm{~mm} \\
(0.0015 \mathrm{in}) \mathrm{TIR}\end{array}$ & $\begin{array}{l}0.04 \mathrm{~mm} \\
(0.0015 \mathrm{in}) \mathrm{TIR}\end{array}$ \\
\hline Velocity & $30,000 \mathrm{RPM}$ & 30,000 RPM & $30,000 \mathrm{RPM}$ \\
\hline Acceleration & $250,000 \mathrm{rad} / \mathrm{sec}^{2}$ & $250,000 \mathrm{rad} / \mathrm{sec}^{2}$ & $250,000 \mathrm{rad} / \mathrm{sec}^{2}$ \\
\hline
\end{tabular}

\section{Output Waveforms}

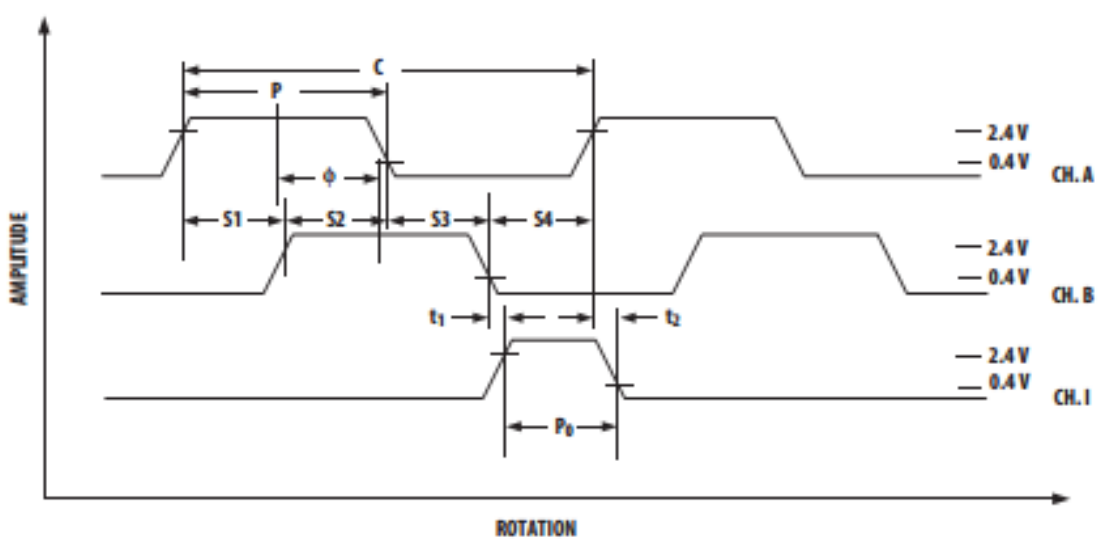




\section{D.3. PCI-6601 National Instrument}

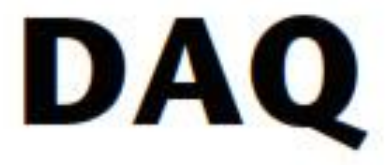

\section{1/6602 User Manual}

High-Speed Counter/Timer

for PCI or PXI ${ }^{\text {Tw }}$ Bus Systems 
Chapter 4 Signal Connections

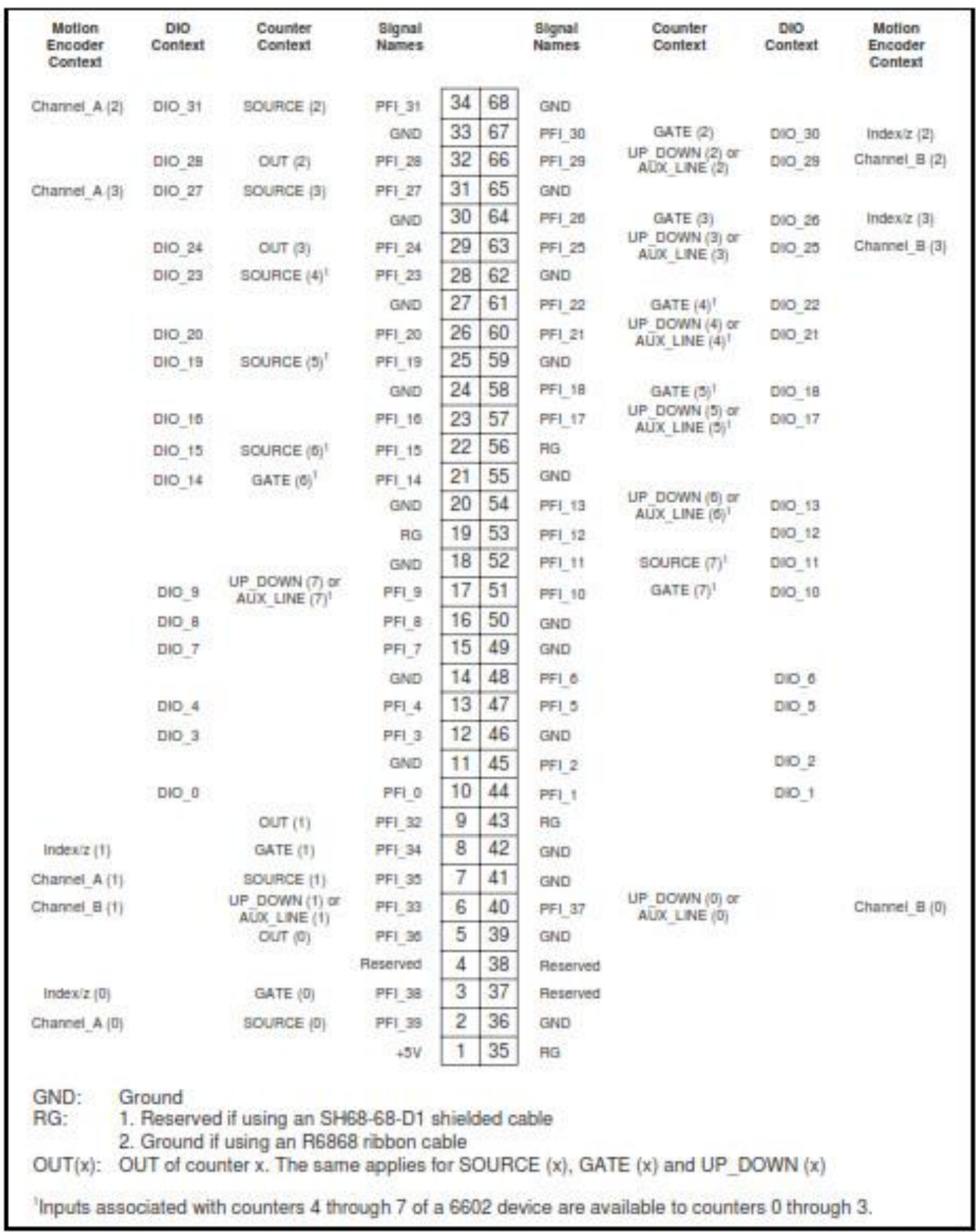

Figure 4-1. Comprehensive Description of PFI Line Functionality for 6601 Devices 


\section{D.4. PCI-6221 National Instrument}

\section{NI 6221}

The following sections contain information about the PCI/PXI-6221

(68-pin), PCI-6221 (37-pin), USB-6221 Screw Terminal, and USB-6221

BNC devices.

\section{PCI/PXI-6221 (68-Pin)}

\section{PCI/PXI-6221 (68-Pin) Pinout}

Figure A-2 shows the pinout of the PCI/PXI-6221 (68-pin) device.

For a detailed description of each signal, refer to the L/O Connector Signal Descriptions section of Chapter 3, Connector and LED Information.

17 Note M Series devices may be used with most E Series accessories. However, some E Series accessories use different terminal names. Refer to the $M$ Series and $E$ Series Pinout Comparison section of Chapter 3, Connector and LED Information, for more information. 
Appendix A Device-Specific Information

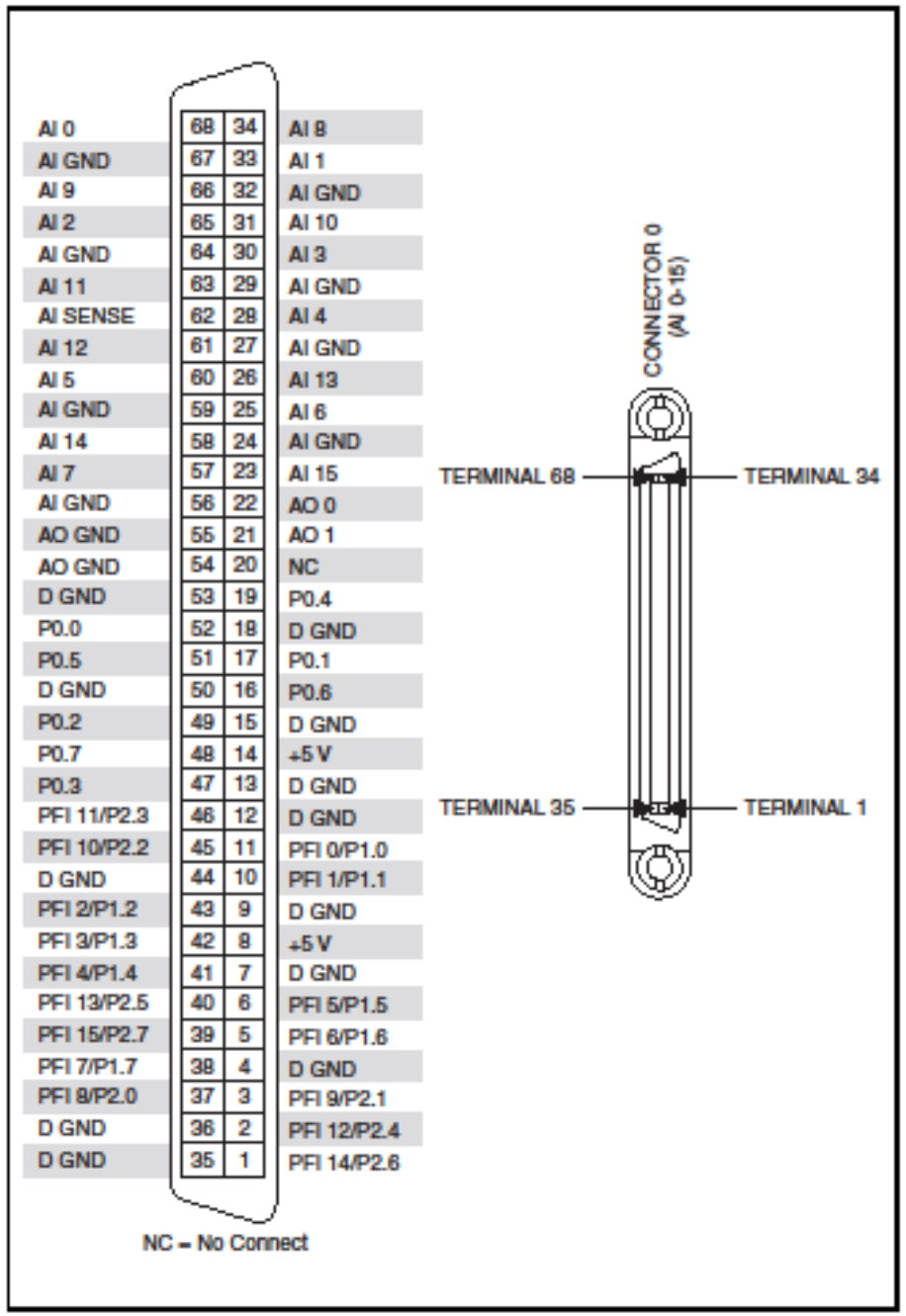

Figure A-2. PCI/PXI-6221 (68-Pin) Pinout 